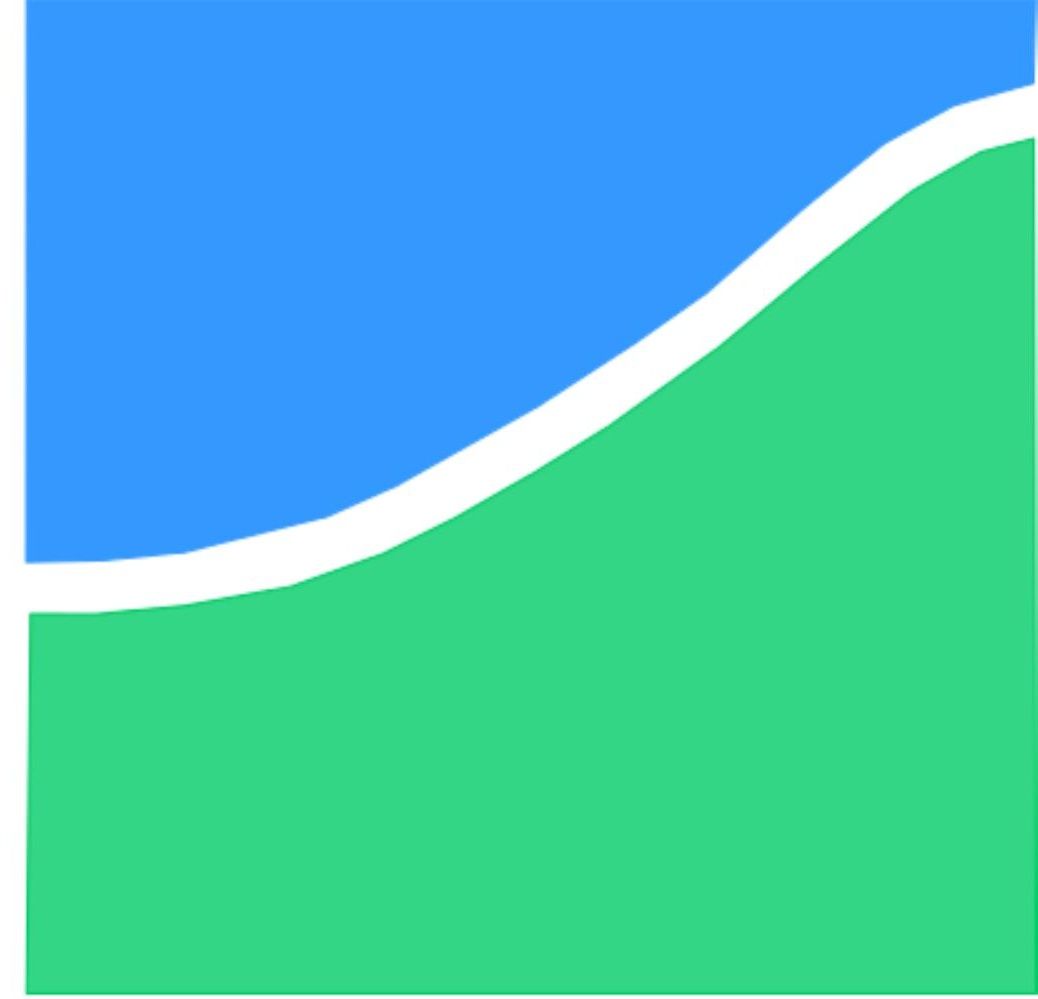

TESE DE DOUTORADO

\title{
SIMULAÇÃO NUMÉRICA DE ONDAS NÃO-LINEARES EM DINÂMICA DOS GASES E RUÍDO DE INTERAÇÃO ROTOR-ESTATOR EM TURBOFANS AERONÁUTICOS
}

Braulio Gutierrez Pimenta

\section{UNIVERSIDADE DE BRASÍLIA}


Ficha catalográfica elaborada automaticamente, com os dados fornecidos pelo(a) autor(a)

Gutierrez Pimenta, Braulio

Numerical Simulation of Nonlinear Waves in Gas Dynamics and Rotor-Stator Interaction Noise of Aeronautic Turbofan Engines / Braulio Gutierrez Pimenta; orientador Roberto Francisco Bobenrieth Miserda; co-orientador Francisco Ricardo da Cunha. Brasília, 2016. $229 \mathrm{p}$.

Tese (Doutorado - Doutorado em Ciências Mecânicas) -- Universidade de Brasília, 2016.

1. Mecânica dos Fluidos. 2. Dinâmica dos Fluidos Computacional. 3. Aeroacústica. 4. Acústica Não Linear. I. Miserda, Roberto Francisco Bobenrieth, orient. II. da Cunha, Francisco Ricardo, co-orient. III. Título. 
UNIVERSIDADE DE BRASILIA

Faculdade de Tecnologia

DOCTORAL THESIS

\section{NUMERICAL SIMULATION OF NONLINEAR WAVES IN GAS DYNAMICS AND ROTOR-STATOR INTERACTION NOISE OF AERONAUTIC TURBOFAN ENGINES \\ Braulio Gutierrez Pimenta}

Doctoral thesis submitted to the Mechanical

Engineering Department as a requirement to obtain

the degree of Doctor of Science in MECHANICAL SCIENCES

\section{Examining Committee}

Prof. Roberto Francisco Bobenrieth Miserda, D.Sc. Committee President, Advisor, ENM/UnB

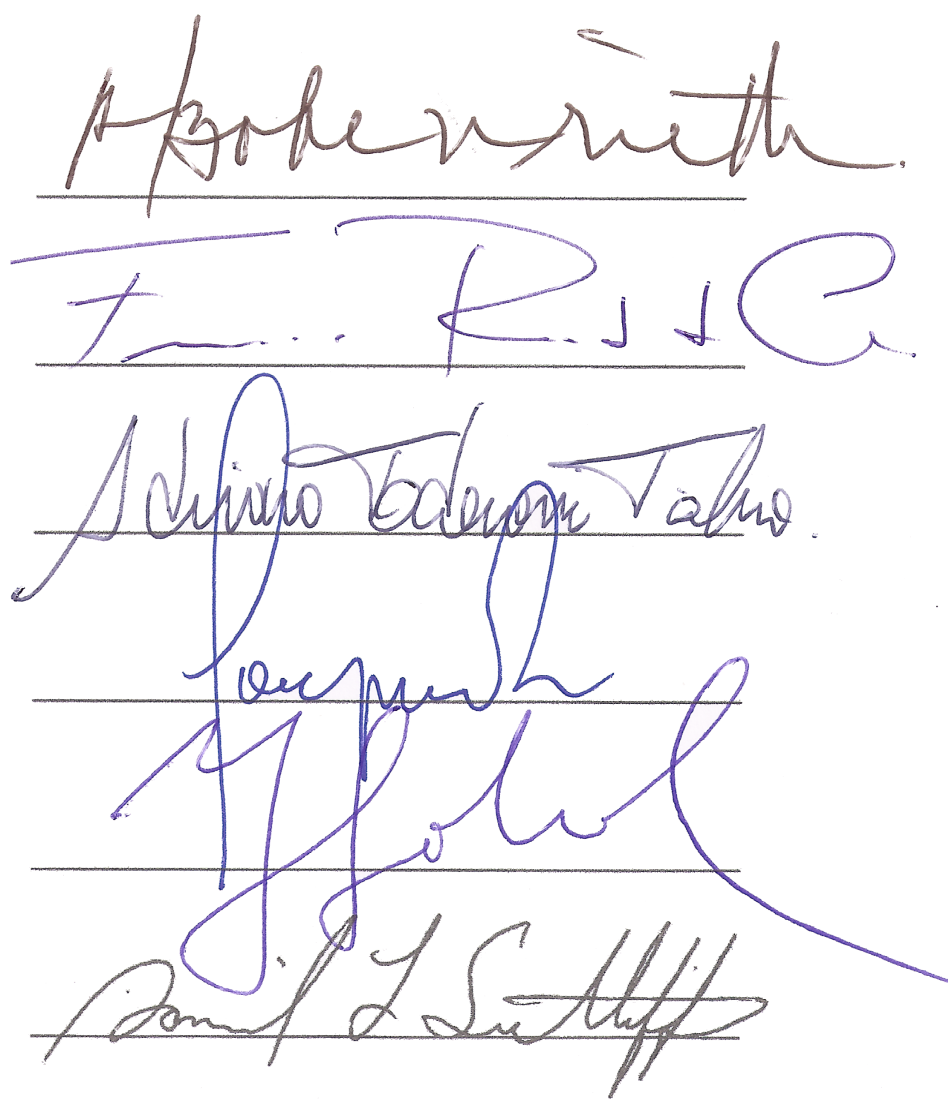

Prof. Francisco Ricardo da Cunha, Ph.D.

Co-Advisor, ENM/UnB

Prof. Adriano Todorovic Fabro, Ph.D.

Internal Examiner, ENM/UnB

Prof. José Luiz Alves da Fontoura Rodrigues, D.Sc.

Internal Examiner, ENM/UnB

Prof. Yuri Dumaresq Sobral, Ph.D.

External Examiner, MAT/UnB

Dr. Daniel Linden Sutliff, Ph.D.

External Examiner, NASA Glenn Research Center 


\section{Dedicatória}

Dedico esta tese ao meu pai José Adib e ao meu avô Braulio. Isto é para vocês.

Braulio Gutierrez Pimenta 


\section{Agradecimentos}

Gostaria de agradecer à minha família, por ter me dado todo o apoio para conseguir terminar este trabalho. Agradeço especialmente minha mãe Julia e meu pai José Adib por garantirem a minha educação e tudo que precisei até aqui, além de terem sido sempre mãe e pai maravilhosos. Agradeço ao meu irmão Reinaldo, por me aguentar desde que nascemos.

Agradeço aos professores da Universidade de Brasília que fizeram diferença na minha formação, em especial ao excelentes professores que tive no grupo VORTEX; Roberto, Francisco Ricardo, José Luiz, Gustavo e Yuri. Agradeço ao meu orientador Roberto Miserda, que além do extraordinário conhecimento técnico e inesgotável vontade, se mostrou uma grande pessoa de amizade inestimável ao longo dos anos em que fui seu aluno. Agradeço ao meu co-orientador Francisco Ricardo que, além de sua amizade, me ensinou o valor de ser incansável na pesquisa e na vida. Agradeço a Daniel Sutliff do NASA Glenn Research Center pela ajuda prestada a este trabalho.

Agradeço a todos os meus amigos que fiz na universidade, em especial aos companheiros de pesquisa Rafael Lobo, Ana Luisa, Nuno, Adriano e Álvaro, por tornarem essa árdua tarefa humanamente possivel. Agradeço também a todos os meus amigos e amigas que fiz durante os anos, esses e essas que dão muita mais graça à vida. Agradeço ao cachorro Bob pela sua companhia e lealdade em todas as horas. Agradeço a todos que de certa forma indiretamente me ajudaram nas horas mais difíceis. Isso inclui escritores e músicos, que com sua arte me ajudaram a manter a humanidade. Agradeço a minha moto Neguinha por fazer tão agradável o percurso diário para a universidade. 


\section{RESUMO}

O presente assunto desta tese se trata de simulações numéricas e novas considerações na modelagem física do ruído gerado por interação do rotor e estator em turbofans aeronáuticos. É feito um estudo teórico preliminar de propagação acústica em dutos com escoamento uniforme, onde é modelada a fonte acústica a partir de preceitos cíclicos da interação rotor e estator. Então são avaliada as características de propagação desses ditos modos de interação, juntamente com a aproximação de dutos anulares finos a ser utilizada nas simulações numéricas, simplificando o grau de liberdade radial do problema de geração e propagação acústica. É feito um estudo teórico da hipótese de escoamento fora do equilíbrio termodinâmico, quando são envolvidas altas frequências características no escoamento e elevadas amplitudes de oscilação. A partir de um modelo pré-existente de viscosidade expansional, é feito um estudo teórico unidimensional no domínio da frequência para baixas amplitudes, em que todos os grupos adimensionais identificados são avaliados na sensibilidade de seus parâmetros. Para o estudo numérico preliminar da viscosidade expansional em condições de propagação unidimensional e no domínio do tempo, é proposto um esquema numérico baseado em diferenças finitas compactas e passo temporal do tipo Runge-Kutta, ambos de alta ordem de precisão numérica. A validação desse esquema numérico é feita com a modificação das equações governantes para se adequar a equação de Burgers viscosa, onde a solução do caso de um salto de descontinuidade é comparada com os valores numéricos obtidos. Modificações também são propostas para o já existente código de simulação numérica VAT (Virtual Aeroacoustic Tunnel), onde um novo esquema de interpolação das faces dos volumes de controle é proposto com argumentos espectrais, visando melhorar as características de propagação do código. Também são propostas modificações no esquema de viscosidade artificial para melhorar sua estabilidade numérica e é feita uma nova implementação do código em CUDA Fortran para utilização de GPUs (Graphical Processing Units) para o cálculo numérico. No estudo unidimensional numérico da viscosidade expansional, é feita a análise de sensibilidade dos parâmetros sobre a dissipação da amplitude da onda estudada. Também é feita uma comparação do decaimento de amplitude de onda para vários valores iniciais de amplitude, em que são comparados os valores numéricos e teóricos e identificada a barreira de linearidade do regime de propagação. São feitas simulações 
bidimensionais de interação rotor e estator com a fronteira imersa móvel que equivalem a aproximação anular e seus resultados são comparados com a teoria desenvolvida nos regimes de escoamento subsônico, transônico e supersônico. Todos os regimes apresentaram excelente concordância teórica no quesito geração e propagação modal e ainda na propagação de ondas de choque em variadas condições geométricas de geração, incluindo a condição realista de ruído de serra elétrica. Uma modificação do modelo de viscosidade expansional é proposta para a sua aplicação em métodos numéricos que efetuem a marcha temporal. Uma primeira aplicação é feita e seus parâmetros testados para o caso supersônico, de elevada amplitude de onda. Os resultados indicaram que o modelo atuou somente no conteúdo relacionado ao divergente do campo de velocidade do escoamento, onde foi mantida a característica dos outros fatores do escoamento, como a vorticidade e a definição dos corpos no escoamento com a metodologia de fronteira imersa móvel.

\section{ABSTRACT}

The subject of this thesis is about numerical simulation and new physical considerations on the noise generated by rotor and stator interaction in aeronautic turbofans. A preliminary theoretical study on noise propagation in ducts with uniform flow was carried, where the acoustic noise source is characterized after cyclic conditions in time and space of the rotor and stator interaction. Then the propagation characteristics of these modes from the noise sources were assessed with the thin duct approximation, simplifications that are used in the numerical simulations, resulting in a reduction of the radial degree of freedom from the noise generation and propagation. A theoretical study of the sound propagation out of the thermodynamic equilibrium was carried out for cases with high frequency and large amplitude waves. A one dimensional theoretical study in frequency domain for waves with infinitesimal amplitude was carried from an already existent expansional viscosity model, where the nondimensional parameters ware identified and their influence on the flow was assessed. For the numerical study of the expansional viscosity in one dimensional flows, a numerical scheme based on compact finite difference for space derivatives and Runge-Kutta time stepping was proposed, both with high order of numerical precision. The validation 
of this numerical scheme was done with some simplifications on the governing equations to result in the viscous Burgers' equation. An exact solution of traveling discontinuity on velocity is compared with the obtained numerical solution. Scheme modifications were proposed for the already existent numerical code VAT (Virtual Aeroacoustic Tunnel), where a new control volume face interpolation based on spectral characteristics to improve the code propagating capabilities was used. Modifications in the artificial viscosity were also proposed to achieve a better numerical stability condition. A code implementation in CUDA Fortran was done to use GPUs (Graphical Processing Unit) in the numerical calculations. On the one dimensional numerical study of the expansional viscosity, a sensitivity analysis of the physical parameters is carried to assess its influence on the wave amplitude decay. Also the decay of several initial wave amplitudes of the numerical results were compared with the theoretical values, and the barrier of the linear propagation regime of initial wave amplitude was identified. Two dimensional cases of rotor and stator interaction were simulated with the moving immersed boundary methodology, where its results were compared with the theoretical ones with the thin duct approximation for the subsonic, transonic and supersonic flow regimes. All the flow regimes were in excellent agreement with the generation and propagation theory of the interaction modes, including shock-wave generation and propagation in several rotor geometric conditions, resulting in the buzz-saw noise. A modification of the expansional viscosity for numerical codes in time domain was proposed. Simulations were carried out and its physical parameters were tested for the supersonic rotor case. Preliminary results indicated that the expansional viscosity model acted only on the velocity divergence related content, where other flow features, such as vorticity and the immersed boundary were kept intact. 


\section{SUMMARY}

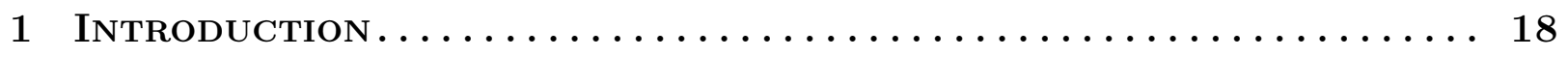

1.1 ECONOMic and Environmental Impact of

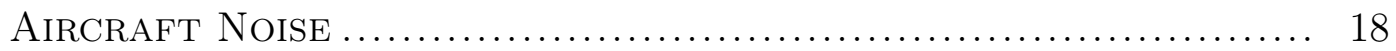

1.2 Aircraft Certification......................................... 19

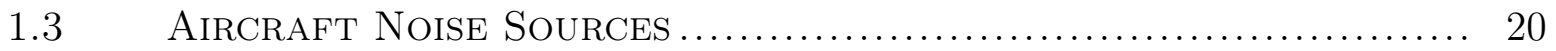

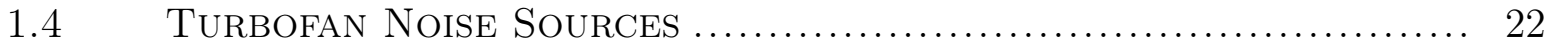

1.5 The Expansional Viscosity ................................... 25

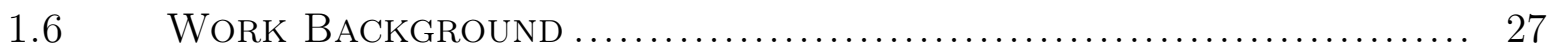

1.6.1 Computational Fluid Dynamics Applied to Aeracoustics ...... 29

1.6.2 Computational EfFiciency .................................. 30

1.6.3 State of the Art of Prediction on Rotor and Stator Inter-

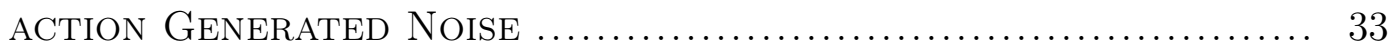

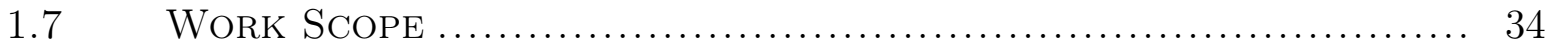

2 Fundamentals of Noise Generation and Propagation in Ducts 38

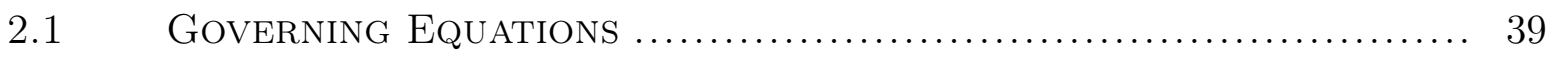

2.2 Noise Generation and Propagation in Ducts with Subsonic NOISE SOURCES ............................................... 43

2.2.1 Wave Equation with Convective Effects Derivation ........... 43

2.2.2 Wave Equation Solution Development ........................ 50

2.2.3 Subsonic Acoustic Source Characterization.................... 58

2.2.4 Tonal Modal Noise Propagation Through the Nacelle-Hub

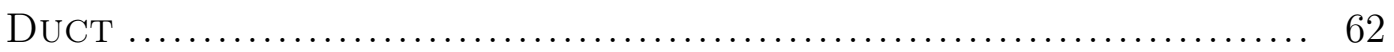


2.3 Noise Generation by Supersonic Sources and Propagation

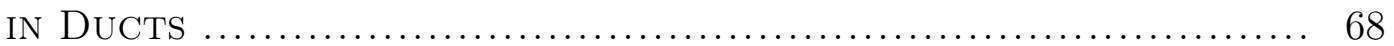

2.3.1 Regular Shock-Waves Propagation in Ducts $\ldots \ldots \ldots \ldots \ldots \ldots \ldots \ldots . . \ldots$

2.3.2 Non-Regular Shock-Waves Propagation in Ducts................ 73

3 Non-linear Gas Dynamics With Expansional Viscosity ....... 77

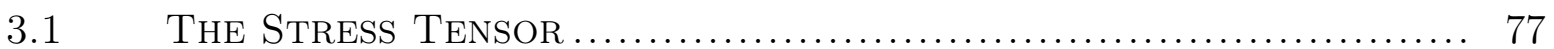

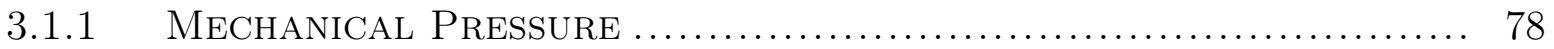

3.1.2 The Stokesian Fluid .......................................... 79

3.1.3 The Non-Stokesian Fluid ..................................... 80

3.1.4 Energy Considerations For the General NeWtonian

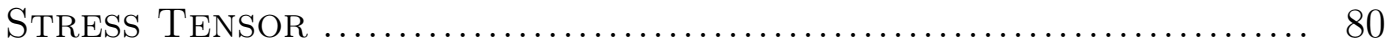

3.1.5 SECOnd Viscosity Coefficient For An Inviscid

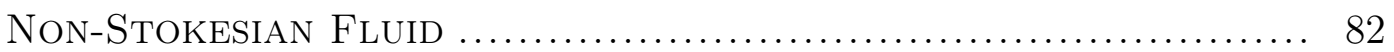

3.2 Linear Stability Analisys of the Governing Equations ........ 88

3.2.1 Non-Dimensional Form of the Governing Equations ............ 88

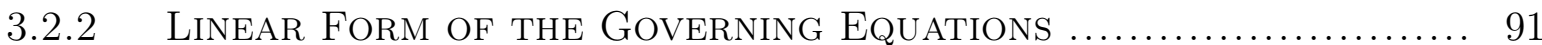

3.2.3 Infinitesimal Perturbations And Its Modal

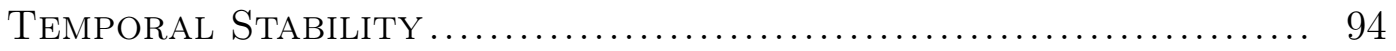

4 Numerical Methodologies ........................... 104

4.1 Expansional Viscosity Numerical Model ......................... 105

4.1.1 Governing Equations ......................................... 105

4.1.2 Numerical Method ............................................ 108

4.1.3 Numerical Method Validation....................................... 117

4.1.4 Implementation Details of the One Dimensional

Expansional Viscosity Numerical Code .......................... 119

4.2 The Virtual Aeroacoustic Tunnel Numerical

Methodology ...................................................... 119

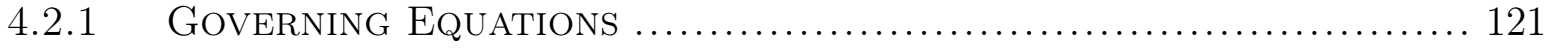

4.2.2 Numerical Method ........................................... 123 
4.2.3 Numerical Imposition of Moving Surfaces by Using the ImMERSEd BOUndARY METHOD ...................................... 137

4.2.4 VAT Code Implementation Details ............................. 143

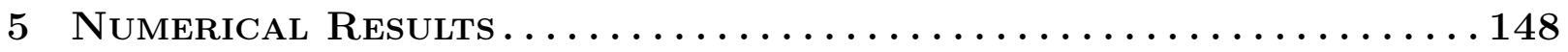

5.1 One Dimensional Simulations With the Expansional ViscosITY MODEL

5.2 Rotor and Stator Cascades Interaction Numerical SimulaTONS

5.2.1 Rotor and Stator Cascades Simulations in Subsonic Regime.. 158

5.2.2 Rotor and Stator Cascades Simulations in Transonic Regime 167

5.2.3 Rotor and Stator Cascade Simulations in SupERSONIC REGIME

5.3 Expansional Viscosity Test in Rotor and Stator Supersonic INTERACTION ................................................... 188

6 Conclusions and Future Work $\ldots \ldots \ldots \ldots \ldots \ldots \ldots \ldots \ldots \ldots \ldots$

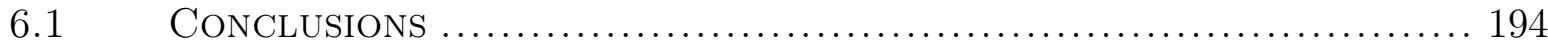

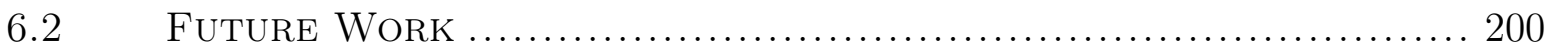

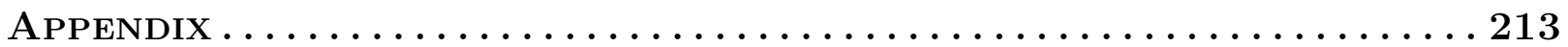

I Bessel and Neumann Eigenvalues $\ldots \ldots \ldots \ldots \ldots \ldots \ldots \ldots \ldots \ldots \ldots$

II Noise Generated Aerodynamically $\ldots \ldots \ldots \ldots \ldots \ldots \ldots \ldots \ldots \ldots \ldots \ldots$

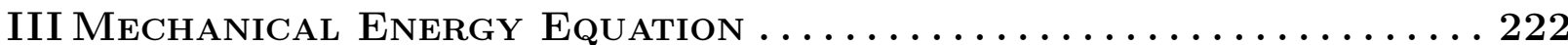

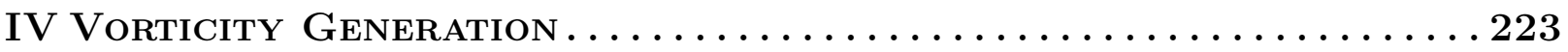




\section{LIST OF FIGURES}

1.1 Certification flying path and the measurement distances of each certification stages [5].

1.2 Certification noise level reduction history and future requirement levels for the future generation of aircraft [11].

1.3 Berton et al. [12] estimates for the $\mathrm{N}+1$ aircraft generation noise sources. It can be noted that the fan would be the major noise source for the three noise certification stages (approach, sideline (lateral) and flyover)....

1.4 NASA conceptual sketch for a $\mathrm{N}+1$ generation aircraft [17].

1.5 Cross section of the Pratt \& Whitney PW1900G turbofan engine, with a high-bypass ratio of 12:1, that will be used on the second generation of the Embraer E-Jets 190 and 195 [15].

1.6 Degeneracy of the sonic-boom wave shape from a supersonic aircraft related to the long distance traveled by the same shock-wave [6]...

1.7 Graphic showing the capabilities and capacity of computational fluid dynamics [23].

1.8 Use of GPUS and co-processors on the world's largest supercomputers [37]... 31

1.9 Proportion of HPC architectures used in the world's largest supercomputers $[37]$

2.1 Cylindrical coordinate system used on the wave equation solution of a duct with the nacelle and the hub. CAD model of the NASA's Active Noise Control Fan rig.

2.2 Thin annular duct approximation. 
2.3 Uniform system of shock and expansion waves generated by the supersonic tangential velocity of the rotor tips. (a) Shock and expansion waves pattern around the rotor blades. (b) Circumferential pressure profile near the rotor blades leading edges.

2.4 Shock-waves and expansion waves distortions due to rotor blade to blade non-uniformity. (a) Shock and expansion waves pattern around the rotor blades. (b) Circumferential pressure profile near the rotor blades leading edges.

2.5 Repeated sawtooth wave pattern generated by supersonic rotor blade cascades. 71

2.6 Geometrical details of the system of regular sawtooth shock-waves at the upstream rotor region [76].

3.1 Physical consistency test of the expansional viscosity model with the following values for the physical parameters: $R e_{\kappa}=1,0, \gamma \beta=1,1, r_{M}=1,05$ e $\omega \tau=1,0.99$

$3.2 \omega \tau$ sensitivity for the following physical parameters: $R e_{\kappa}=1,0$, $\gamma \beta=1,1, r_{M}=1,05$.

3.3 Expansional Reynolds number $R e_{\kappa}$ sensitivity for the following physical parameters: $\gamma \beta=1,1, r_{M}=1,05, \omega \tau=1,05$.

3.4 Velocity ratio $r_{M}$ sensitivity for the following physical parameters: $\gamma \beta=1,1$, $R e_{\kappa}=1,0, \omega \tau=1,05$.

3.5 Material constants $\gamma \beta$ sensitivity for the following physical parameters: $R e_{\kappa}=1,0, r_{M}=1,05, \omega \tau=1,05$.

4.1 Transfer function $F(k)$ used for filtering of the poorly resolved wavenumber content. ...

4.2 Mesh convergence of the proposed numerical scheme for the traveling wave with discontinuity solution of the Burgers' equation for several Reynolds numbers.

4.3 Spectral resolution of several numerical interpolation schemes. Note the amplification region from the Ducros' scheme, while the formal fourth order scheme has an asymptotic behavior around the unity value. 131

4.4 Mesh stencil for derivative calculation on vertex $i, j[117]$. 136 
4.5 Basic architecture of a general purpose graphic computing unit (GPGPU) $[119]$

5.1 Illustrative shockwave formation due to non linear effects directly associated with the wave finite amplitude. The variable shown is the linear momentum $\rho u$.

5.2 Linearity level of the theoretical and numerical amplification factor comparison.153

5.3 Wavenumber $k$ sensivity for three order of magnitude with the following parameters: $\operatorname{Re}_{\kappa}=0,5, \Psi=1,0, \beta=1,0, \gamma=1,4$ e $r_{M}=1,05$.

5.4 Ratio of flow and fluid relaxation characteristic times $\Psi$ sensivity with the following parameters: $R e_{\kappa}=0,5, k=1,0, \beta=1,0, \gamma=1,4$ e $r_{M}=1,05$. . .

5.5 Expansional Reynolds number $R e_{\kappa}$ sensivity with the following parameters: $\Psi=1,0, k=1,0, \beta=1,0, \gamma=1,4$ e $r_{M}=1,05$.

5.6 Ratio of propagation speeds in and out of the thermodynamic equilibrium $r_{M}$ sensivity with the following parameters: $\Psi=1,0, k=1,0, \beta=1,0$, $\gamma=1,4$ e $R e_{\kappa}=0,5$

5.7 Rotor blade (red) and stator vane (blue) geometries. 158

5.8 Computational domain of the regular cartesian mesh. 159

5.9 Grid resolution at the leading edge of the blade.

5.10 Visualization of the instantaneous aeroacoustic field generated by the rotor cascade.

5.11 Spectra of the RMS value of the pressure fluctuation for the near-field (blue) and far-field (red) static probes.

5.12 Visualization of the instantaneous aeroacoustic field $\beta_{T}$ generated by the interaction of the rotor and stator cascades.

5.13 Spectra of the RMS value of the pressure fluctuations for a static far-field probe (red) and a moving far-field probe (blue) showing the mode decomposition around each harmonic. 166

5.14 Computational domain with a regular Cartesian mesh.

5.15 Visualization of the instantaneous aeroacoustic field generated by the transonic rotor cascade using the variable $\beta_{T}$. 
5.16 Spectra of the RMS value of the pressure fluctuations for the static nearfield probe 1 (blue) and the moving nearfield probe 1 (red) showing the decomposition of the nonlinear modes around each harmonic.

5.17 Nearfield probe pressure RMS signal decay due to the shock-waves evanescence.170

5.18 Pressure spectra of the farfield moving probe and the static probe, showing the modal decompostions up to the fourth harmonic.

$5.19 \beta_{T}$ flowfield variable visualization with the modal angle measurement of the propagating wave fronts.

5.20 Spectra of the RMS value of the pressure fluctuations for the moving interstage probe (red) and the moving nearfield probe 1 (blue) showing the mode decomposition around each harmonic, being all the identifiable modes given by the Tyler-Sofrin rule. The light-blue regions delimited by dashed lines are the cut-on regions around each harmonic.

5.21 Linear, transition and nonlinear regions of the computational domain. The dominant linear modes $\mathrm{m}=-10$ and $\mathrm{m}=+3$, associated to the first harmonic, are easily identified in the linear region. In the transition region it is possible to observe the superposition phenomena of nonlinear waves that will result in the linear modes. Transonic shocks and shock-wake interactions are observed in the nonlinear region.

5.22 Computational domain of regular control volumes showing the rotor blades and stator vanes cascades positioning, the pressure probes and circumferential measuring locations.

5.23 The $\beta_{T}$ flow visualization of the supersonic nominal rotor blade cascade alone configuration.

5.24 Spectra up to the fourth harmonic of pressure probes from the supersonic nominal rotor blades cascade only case.

5.25 Pressure coefficient profiles of the circumferential stations for the supersonic nominal rotor blades cascade only configuration.

5.26 Pressure coefficient decay of the sawtooth pattern in the $\mathrm{x}$ direction for the supersonic nominal rotor blades only configuration.

5.27 The $\beta_{T}$ visualization of the supersonic nominal rotor blades and stator vanes cascades configuration. 
5.28 Spectra up to the fourth harmonic of pressure probes from the supersonic nominal rotor blades and stator vanes cascades case.

5.29 Pressure coefficient profiles of the circumferential stations for the supersonic nominal rotor blades and stator vanes cascades configuration.

5.30 Pressure coefficient decay of the sawtooth pattern in the $\mathrm{x}$ direction for the supersonic nominal rotor blades and stator vanes cascades configuration.

5.31 The $\beta_{T}$ visualization of the supersonic realistic rotor blades cascade configuration.

5.32 Spectra for the first harmonic of pressure probes from the rotor blades cascade case showing the multiple tone noise generation from shaft order 1 to 16 , being the later equal to the blade passing frequency.....

5.33 Numerical and theoretical pressure coefficient profiles of the circumferential stations for the supersonic realistic rotor blades cascade configuration.

5.34 The $\beta_{T}$ visualization of the supersonic realistic rotor blades and stator vanes cascades configuration.

5.35 Spectra for the first harmonic of pressure probes from the supersonic rotor blades and stator vanes cascades case showing the multiple tone noise generation from shaft order 1 to 16, being the later equal to the blade passing frequency.

5.36 Pressure coefficient profiles of the circumferential stations for the supersonic realistic rotor blades and stator vanes cascades configuration.

5.37 Comparison of the decay rate of the shock-waves intensity for the supersonic rotor and stator cascade interaction for several expansional viscosity parameter cases and a base case.

5.38 The $\beta_{T}$ visualization of the base case and the cases with expansional viscosity. Upper left $k_{\text {exp }}=0,0$, upper right $k_{\text {exp }}=10^{-4}$, lower left $k_{\text {exp }}=10^{-3}$, lower right $k_{\text {exp }}=10^{-2}$.

5.39 The $\beta_{T}$ visualization of the rotor blades and stator vanes interaction region. Upper left $k_{\text {exp }}=0,0$, upper right $k_{\text {exp }}=10^{-4}$, lower left $k_{\text {exp }}=10^{-3}$, lower right $k_{\text {exp }}=10^{-2}$. 


\section{LIST OF TABLES}

4.1 Runge-Kutta coefficients for scheme RK5(4)9(2R+)M.................... 115

5.1 Theoretical and numerical comparison of the initial wave amplitude $u_{a} \ldots \ldots .152$

5.2 Theoretical cut-on modes and and wave-front angles from the first to the

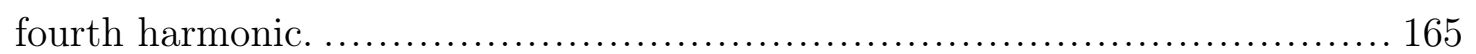

5.3 Theoretical cut-on modes from the first to the fourth harmonic for the transonic rotor and stator cascade interaction case.......................... 171

5.4 Randomly generated stagger error distribution for the rotor blades cascade in realistic conditions.

I.1 Numerical values of $\sigma_{m n}$ for $R_{i} / R_{o}=0.0$ (circular duct) and $R_{i} / R_{o}=0.5$ (annular duct). ....................................................... 215

I.2 Numerical values of $\sigma_{m n}$ for $R_{i} / R_{o}=0.9, R_{i} / R_{o}=0.95$ and $R_{i} / R_{o}=1.0 \ldots 216$ 


\section{LIST OF SYMBOLS}

a Runge-Kutta method coefficient

$a_{n} \quad$ Explicit stencil coefficient

$\alpha \quad$ Angle of attack

$\alpha_{m} \quad$ Compact stencil coefficient

$b \quad$ Runge-Kutta method coefficient

$B \quad$ Number of rotor blades

$\beta \quad$ Flow angle of the rotor chord or the barotropic relation constant

$\beta_{T} \quad$ Temperature gradient visualization variable

$c_{p} \quad$ Specific heat at constant pressure

$c_{v} \quad$ Specific heat at constant volume

c $\quad$ Sound speed

D Rate of strain tensor

$\Delta t \quad$ Numerical time step

$\delta_{i j} \quad$ Kronecker Delta function

$\delta \quad$ Dirac Delta distribution

$\Delta \quad$ Finite variation

d Artificial dissipation operator applied to a control volume face

$\mathcal{D} \quad$ Sum of the artificial dissipation operators applied to a control volume face

$\eta \quad$ Isotropic viscosity tensor or damping factor

$e \quad$ Specific internal energy

$e_{k} \quad$ Specific kinetic energy

$e_{t} \quad$ Specific total energy

$\epsilon^{(n)}$ Artificial dissipation coefficient of order $n$

E $\quad$ Flux vector on $x$ direction 
F Flux vector on $y$ direction

$f \quad$ Frequency

$f_{i} \quad$ Volumetric pseudo-force

$\mathcal{F} \quad$ Flux function

$\gamma \quad$ Specific heat ratio or barotropic relation constant

G Flux vector on $z$ direction

$h \quad$ Harmonic number

$H \quad$ Heaviside step function

$i \quad$ Imaginary number $\sqrt{-1}$

i $\quad$ Unity vector on $x$ direction

I Identity tensor

$j \quad$ Alternate symbol for the imaginary number $\sqrt{-1}$

j Unity vector on $y$ direction

$J_{m} \quad$ Bessel function of order $m$

$\mathbf{k} \quad$ Unity vector on $z$ direction

$\kappa \quad$ Second viscosity coefficient

$k \quad$ Integer value, wavenumber or parametric constant

$K \quad$ Wave number of the separated solutions

$K^{(n)}$ Artificial dissipation constant of order $n$

$L \quad$ Characteristic length

$\lambda \quad$ Wave length or expansional modulus

$m \quad$ Circunferential mode

M Mach number or compact stencil size

$\mu \quad$ First viscosity coefficient or shock-wave Mach angle

$N \quad$ Stencil size of the noncompact side

$N_{m} \quad$ Neumann function of order $m$

$N_{v} \quad$ Number of control volumes of the computational mesh

n Unit vector on the normal direction

$\nabla \quad$ Differential operator

$p \quad$ Pressure

$P \quad$ Mode amplitude 
$\phi \quad$ Shock-wave angle in respect to the axial direction

$\phi_{i} \quad$ Shock-wave front normal angle in respect to the $i$ coordinate

$\varphi \quad$ Rotor blade and pressure probe velocities ratio

$\Pi \quad$ Flux tensor

$\Psi \quad$ Artificial sensor numerical sensor or non-dimensional parameter of characteristic time

$q_{s} \quad$ Volumetric flux

q Heat flux vector

$\rho \quad$ Density

$r \quad$ Radial coordinate

$r_{M} \quad$ Propagation velocities ratio inside and outside the thermodynamic equilibrium

$\mathcal{R} \quad$ Gas constant

$R \quad$ Separated radial function

$R_{i} \quad$ Hub radius

$R_{o} \quad$ Nacelle Radius

R Left hand side vector of the governing equations

$S \quad$ Surface

S Surface vector

$s \quad$ Specific entropy

$s_{x} \quad$ Surface vector component on the $x$ direction

$s_{y} \quad$ Surface vector component on the $y$ direction

$s_{z} \quad$ Surface vector component on the $z$ direction

$\sigma \quad \mathrm{Hub}$ and nacelle radii ratio

$\sigma_{m n}$ Bessel and Neumann eigenvector

$T$ Temperature, spectral transfer function or time separated function

$t \quad$ Time coordinate

$\theta \quad$ Angular coordinate

$\Theta \quad$ Angular coordinate separated function

t Tangential unity vector

$t_{a}$ Acceleration time from the stagnating to the developed flow condition

$\tau_{i j} \quad i j$ component of the stress tensor

$\tau \quad$ Stress tensor relaxation time 
$U \quad$ Velocity magnitude

U Conservative variables vector

$u \quad x$ direction velocity component

u Velocity vector

$u_{i} \quad i$ direction velocity component

$V \quad$ Volume or number of stator vanes

$v \quad y$ direction velocity component

$w \quad z$ direction velocity component

$\omega \quad$ Time solution argument

$\Omega \quad$ Rotor shaft angular frequency

$x \quad$ First space coordinate

$X \quad$ Separated function of the axial coordinate

$\otimes \quad$ Tensor product operator

$x_{i} \quad i$ space coordinate

$\xi \quad$ General thermodynamic quantity

y Second space coordinate

$z \quad$ Third space coordinate or pressure jump across shock-wave

Subscript

$B$ Rotor blade

ref Refernce value

$n \quad$ Normal direction or numerical value

$t$ Tangential direction or theoretical value

0 Mean value or inside thermodynamic equilibrium

bp Related to rotor passing frequency

$\infty \quad$ Value outside the thermodynamic equilibrium

$i \quad$ Initial value

$m \quad$ Shock-wave jump mean value

rel Relative value

$u \quad$ Related to the propagation duct wall

$b \quad$ Boundary volume

exp Expansional viscosity 
$\mathrm{Pb} \quad$ Rotor pitch

$T$ Related to the temperature

a Related to the wave amplitude

c Critical value

\section{Superscript}

* Non-dimensional value

I Variable fluctuation or function derivative

$n \quad$ Time step

$b \quad$ Boundary volume

- Mean value of the control volume

$\sim \quad$ Favre mean of the control volume

- Instant time variation

$T$ Tensor transpose 


\section{Chapter 1}

\section{Introduction}

\subsection{Economic and Environmental Impact of Aircraft Noise}

Noise has been an omnipresent pollution, though often underestimated. Even for small exposure periods, several effects on human health may appear such as: increase in blood pressure, sleep disturbances [1], cognitive skills development problems in small children [2] and several other psychiatric problems [3]. A broad range of machines produce noise that can affect in a serious way the human health, including airplanes and helicopters.

An increase in noise causing health issues comes from the technological and economic development that took place from the 1960's. After the successful introduction of the jet engines in commercial aviation; transport and passengers aircraft, air transport became cheaper, increasing the flight volume and thus the areas affect by aircraft noise.

Aware of this problem, national and international regulation agencies began to impose rules and restrictions on airports that were located close to residential areas. Since these restrictions were not enough to control the noise levels, the models of aircraft that can operate at a given airport were restricted. Certification processes were implemented on new models of aircraft, where a maximum permissible noise level was allowed for a given class of aircraft.

In addiction, some regions with nearby airports suffered economic and social impacts, 
including airspace reconfiguration, operating hours restrictions for some airports and even relocating housing complexes.

\subsection{Aircraft Certification}

Aircraft noise certification is basically a process in which aircraft manufacturers are required by regulating agencies to prove that their products fulfill some noise specification requirements. Noise levels are specified by measuring the noise radiated from the aircraft with microphones located at certain positions on the ground. The measurement process takes place with the aircraft flying through reference points and under certain conditions. The certification process is made in three rating points [4] that simulate the aircraft operating conditions:

1. Approach measurements: landing conditions, measurement points located through the center line of the runway located at two kilometers from the runway end and 120 meters of altitude with an approach angle of 3 degrees.

2. Lateral measurements: take-off operating condition, with engines at full power and flying altitude defined by the noise level measured at the previous stage. The noise measurement is made with an array of microphones located through two lines in parallel and at each side of the runway, at 450 meters from its center line. The noise level to be considered is from the microphone pair that measured the maximum noise level from both lines. The final certification noise level is the mean value from these two microphones.

3. Flyover measurement: climbing conditions right after the take off. The noise measurement is made at a distance of 6500 meters from the point where the aircraft was airborne.

After the measurements are made, the measured noise levels at the three stages are compared with the regulations according to the aircraft type. If at any of the certification points the aircraft has higher a noise level than the allowed one, the aircraft fails the certification process and it cannot operate at civilian airports. 


\section{Trajectory and Certification Locations}

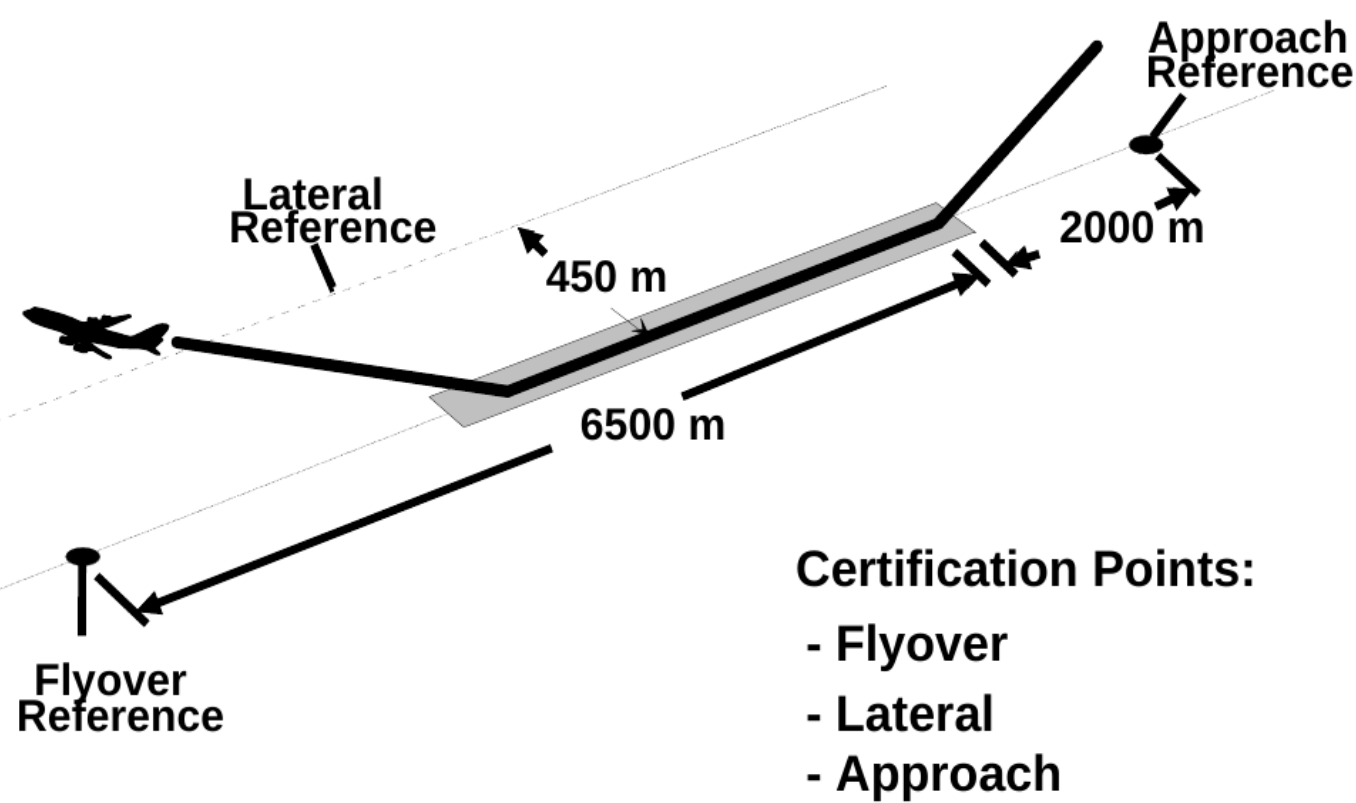

Figure 1.1: Certification flying path and the measurement distances of each certification stages [5].

It is worth to note that regularly, both International Civil Aviation Organization (ICAO) and the Federal Aviation Administration (FAA) are reducing the allowed noise levels from the certification process, putting aircraft manufactures at even more difficult conditions, as observed in figure 1.2. For this reason, it is estimated that one billion dollars are spent annually [6] to research new technologies that may reduce aircraft noise, not only by the aerospace industry, but by several government agencies as well, such as NASA [7], DLR [8], ONERA [9] and JAXA [10].

\subsection{Aircraft Noise Sources}

Aircraft noise sources can be categorized into two major groups: noise generated by propulsion systems and noise generated by the aircraft airframe. In propulsion systems that make use of turbofan engines the main noise sources are the fan, the jet and the engine core (composed of the compressor, the turbine and the combustion chamber). The 


\section{New Technology Enables Aircraft To Meet Future Requirements}

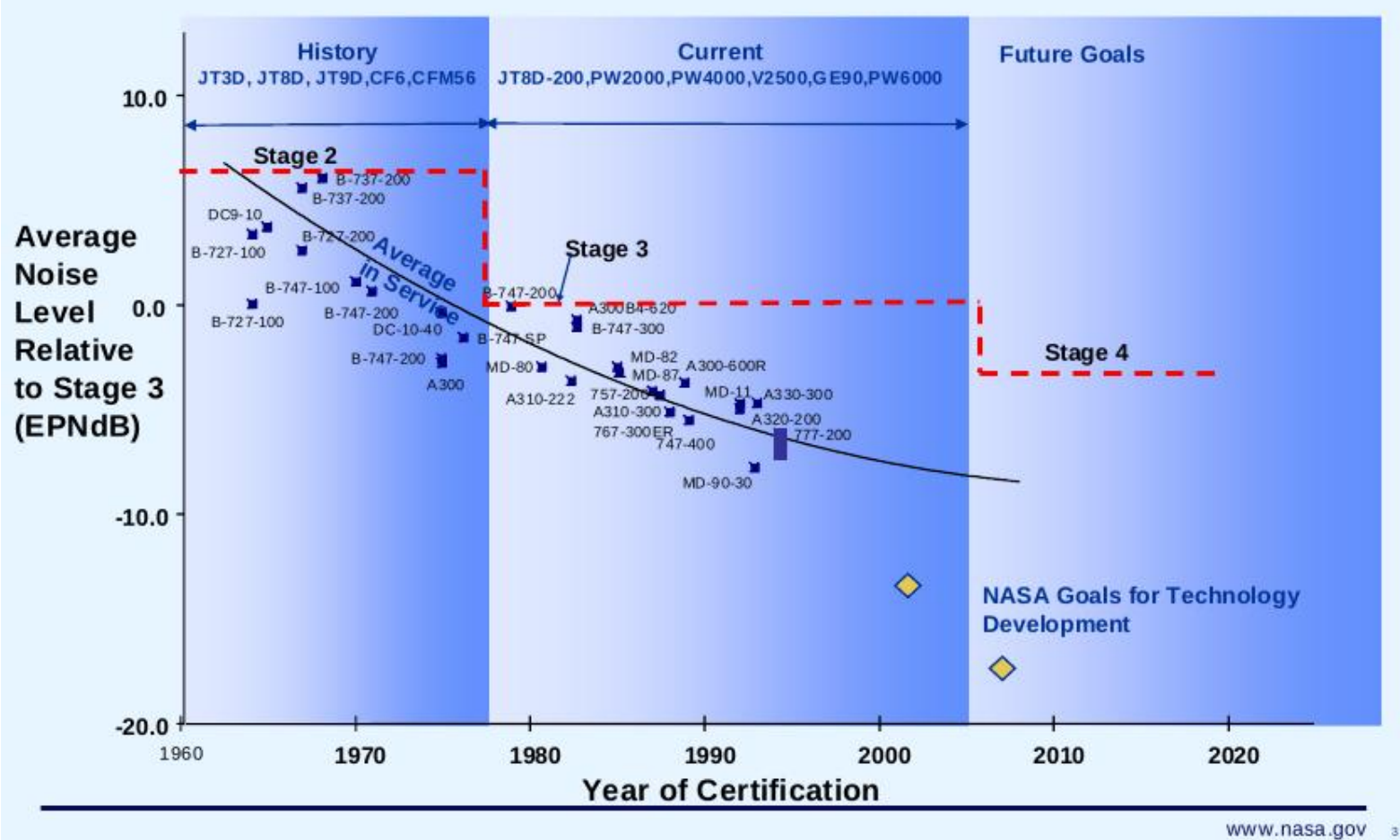

Figure 1.2: Certification noise level reduction history and future requirement levels for the future generation of aircraft [11].

airframe main noise sources are the landing gear, the high-lift devices (flaps and slats) and the induced noise from the wing trailing edge.

Estimatives [12] were made of the noise sources contribution to the overall noise level for the next generation of aircraft $(\mathrm{N}+1$ generation) that are planned for certification process in 10 years [13]. These estimates are shown in figure 1.3 for the three noise certification points. It is worth to mention that it is expected that for the future generation of aircraft, the fan noise will have the highest noise level for all the three certification points. The causes are many: reduction from airframe noise due to new conceptual ideas, as shown in figure 1.4, and by the increase of the turbofan bypass ratio [14] in modern engines, where bypass ratios bigger of 10:1 will be common in the near future. 


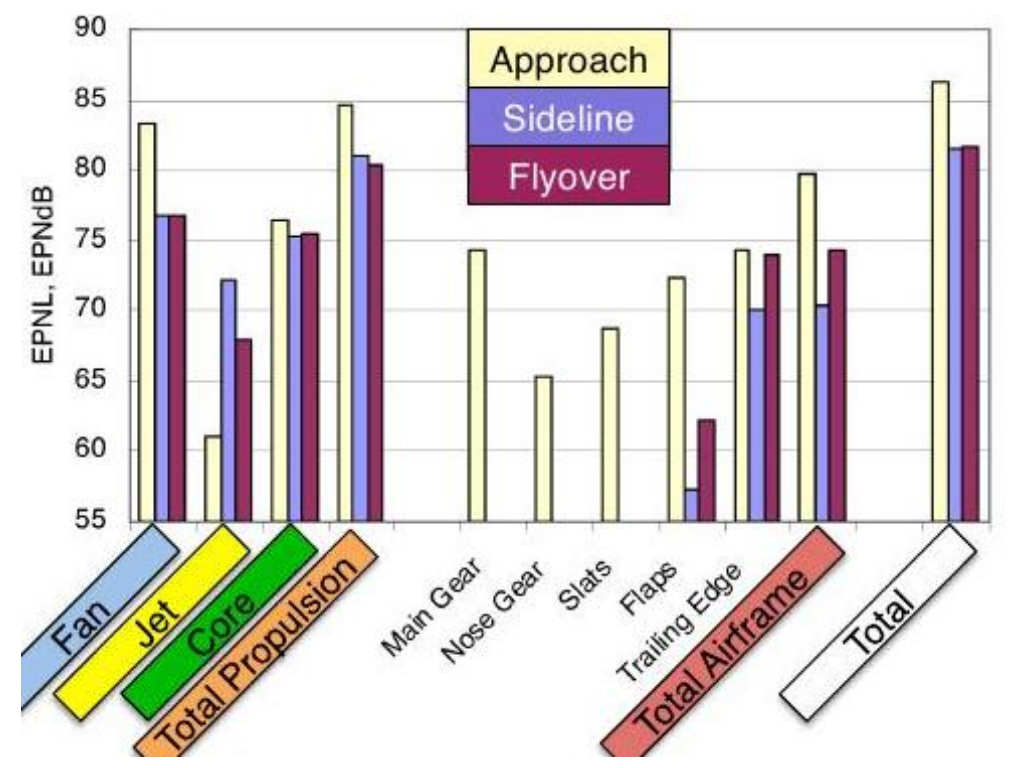

Figure 1.3: Berton et al. [12] estimates for the $\mathrm{N}+1$ aircraft generation noise sources. It can be noted that the fan would be the major noise source for the three noise certification stages (approach, sideline (lateral) and flyover).

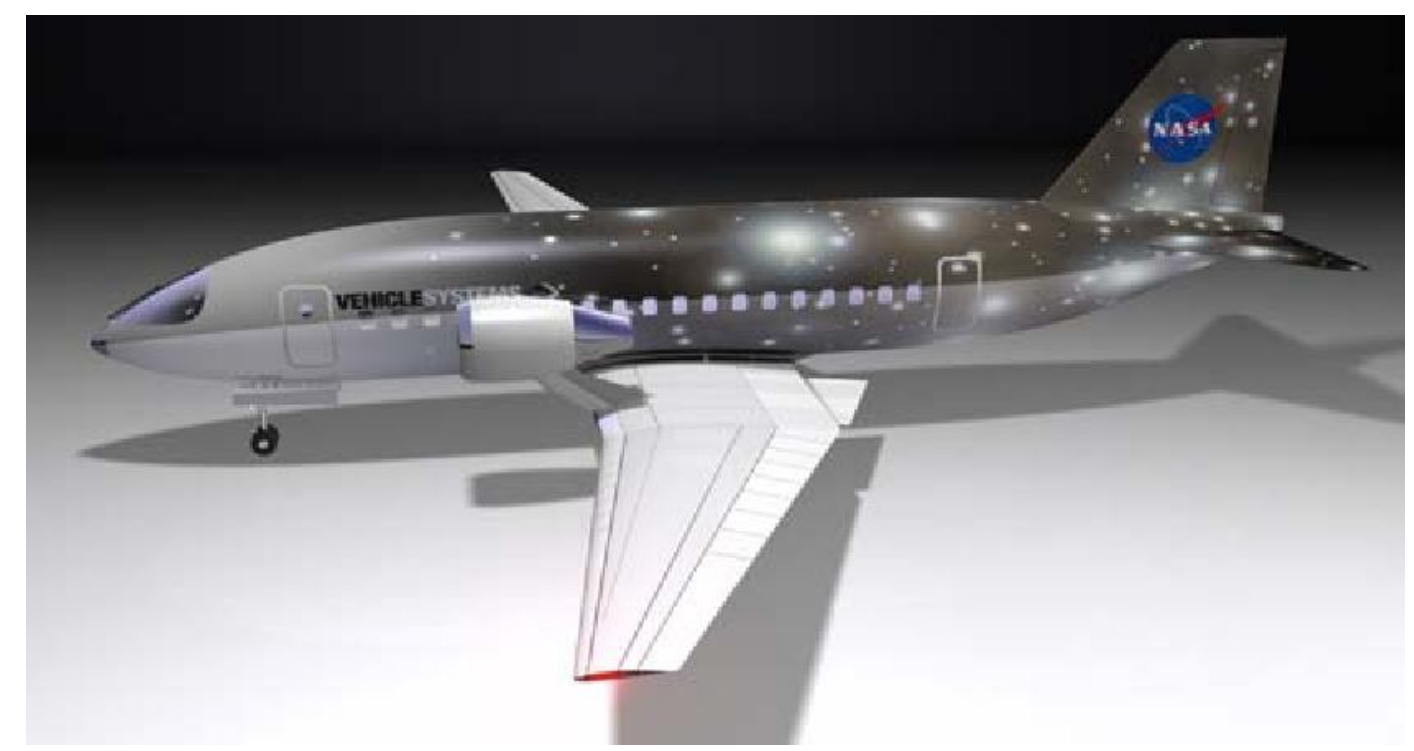

Figure 1.4: NASA conceptual sketch for a $\mathrm{N}+1$ generation aircraft [17].

\subsection{Turbofan Noise Sources}

Figure 1.5 shows a Pratt \& Whitney PW1900G [15] turbofan engine cross section, with a ultra-high bypass ratio of 12:1, that will be used on the second generation of the Embraer E-Jets 190 and 195 [16]. This turbofan engine is also used in the Bombardier CSeries, 
Airbus A320neo and Irkut MC-21.

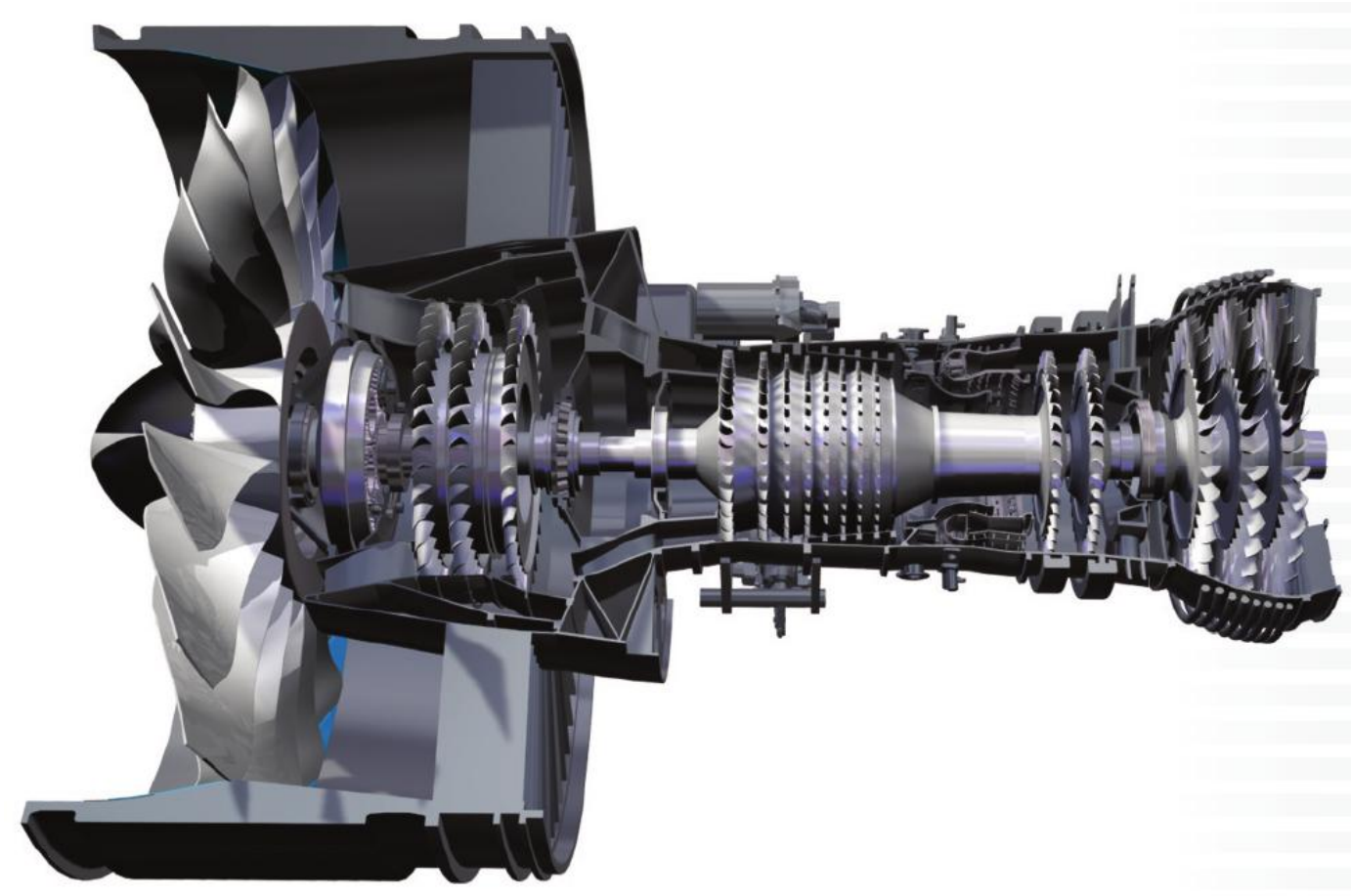

Figure 1.5: Cross section of the Pratt \& Whitney PW1900G turbofan engine, with a highbypass ratio of 12:1, that will be used on the second generation of the Embraer E-Jets 190 and $195[15]$.

In figure 1.5, from the fan inlet to the fan exhaust, i.e., along the flow direction, the engine components can be seen: the fan, the core components; the three low pressure compressor stages, the eight stages of high pressure from the turbine. Outside the core, the stator vanes can be seen, located at the same axial position as the low-pressure compressor second stage.

It is worth mentioning that both the turbine and the low pressure compressor of this turbofan engine spin at the same angular speed, but the fan rotor spins at a third of this, due to a gearbox located between the fan rotor and the low pressure compressor. The main function of the rotor is to perform work over the fluid that passes through it, raising the air pressure and its circumferential speed.

After its passage through the fan, the air flow is divided into a $1 / 13(7,7 \%)$ that passes to the core to be used in the combustion process, allowing power to be generated by the turbines and used by the compressors. The remaining $12 / 13(92,3 \%)$ of the air flow goes 
through the bypass stator vanes, where the circumferential speed component induced by the rotor blades is transformed into pressure, that will finally be transformed into axial speed component, and therefore, generating most of the engine total thrust.

As can be observed in figure 1.5, the rotor blades and the stator vanes sizes are much bigger than the stator vanes and rotor blades from the compression stages of the core. This difference, along with the 12:1 air mass proportion that passes through the rotor blades and the core, explains the fact that the fan noise tends to rise with the increase of the fan bypass ratio. The increase of this ratio also helps the noise dominance of the fan noise over the jet noise, because with a higher bypass ratio, the jet speed will be smaller, making the turbofan more propulsion efficient and lowering the overall generated noise.

The noise sources with the highest noise level in high-bypass turbofan engines are, in decreasing order of importance, the following:

- Rotor blades and stator vanes interaction: pressure, wake and vorticity fields generated by the rotor blades interact with the stator vanes, generating noise that has both broadband and tonal content in the spectrum. The generated tonal noise is directly associated with the cyclic nature of the interaction, at the blade passing frequency and its harmonics. The broadband noise spectrum is associated with the non cyclic component of this interaction.

- Buzzsaw noise: at the full power condition of a turbofan engine, found in the sideline noise certification stage, the rotor blade tips are at supersonic flow regime, resulting in a system of shock-waves that propagates through the nacelle duct to the inlet direction. Slight differences occur from blade to blade geometric manufacturing tolerances and due to extended use, generating tonal noise and its harmonics at sub-harmonics of the rotor passing frequency (shaft orders). Also called multiple pure tone (MPT) noise.

- Rotor self induced noise: the physical mechanism responsible for the self induced noise in airfoils is the same for rotor blades, with the mixing boundary layers of the suction and pressure sides of the rotor sides. At the turbofan engine operating conditions this noise has a broadband like spectrum. 
- Interaction between the nacelle boundary layer and the rotor blades tip. The generated noise has a broadband like spectrum, given its non cyclic nature.

- Turbulence ingestion: atmospheric turbulent eddies are ingested by the fan and interact with the rotor blades, resulting in broadband noise content, due to the wide spectrum of the Kolmogorov cascade turbulence scales.

For a high bypass turbofan engine, the broadband noise produced by the rotor blades and stator vanes interaction is typically $10 \mathrm{~dB}$ higher than the other sources of broadband noise described above. Given by the decibel scale, it means that the acoustic power (sound power level) associated with the noise generated by the rotor-stator interaction is at least 10 times bigger than the acoustic power of the other fan noise sources.

\subsection{The Expansional Viscosity}

There are cases in which the acoustic propagation phenomena does not necessarily occur in thermodynamic equilibrium. In processes at a molecular level that have not achieve homogeneity, the molecular relaxation cannot go at the same pace as flows with high gradients. At non equilibrium states the thermodynamic characteristics of the environment may differ significantly from the state of equilibrium.

The acoustic generation, propagation and radiation phenomena may be concerned with high frequencies, propagation at huge distance or high amplitude interactions. It is inferred that such conditions of the fluid are not totally in thermodynamic equilibrium or that its effects are not negligible either. Physically speaking, the non equilibrium effects of such phenomena implies that the relaxation time of the fluid is much higher than the characteristic time of the flow, or that even when only small effects are considered, the period or field of influence is relatively large enough.

An important case of large expansion and compression gradients is in the rotor blades and stator vanes in a turbofan. A good case to be compared with is the compression and expansion of a given gas inside a chamber with a sliding piston in it. The gas internal energy increases as the piston transmits energy by means of a work done by compression of the fluid. The collision of the air molecules with the piston distributes the energy through the 
different modes of translation, rotation and vibration, where in some modes this transfer is faster than in others, like in the translating mode parallel to the piston moving direction. The energy transfer to the modes of the other two direction takes more time, characterizing the thermodynamic equilibrium when the modes have statistically the same energy. The vibrating and rotational modes also have their characteristic time to achieve equilibrium [18]. These relaxation times may differ significantly when fluids of several species are considered. Relaxation effects related with the translation, vibration and rotation modes of molecules of a gas are important and may not be neglected in situations that the gas is under severe changes of pressure, like in cases where there are high frequency waves or shock-waves over the flow [19]. Those flow conditions are easily found in the rotor and stator interaction region of turbofan engines during some flight stages.

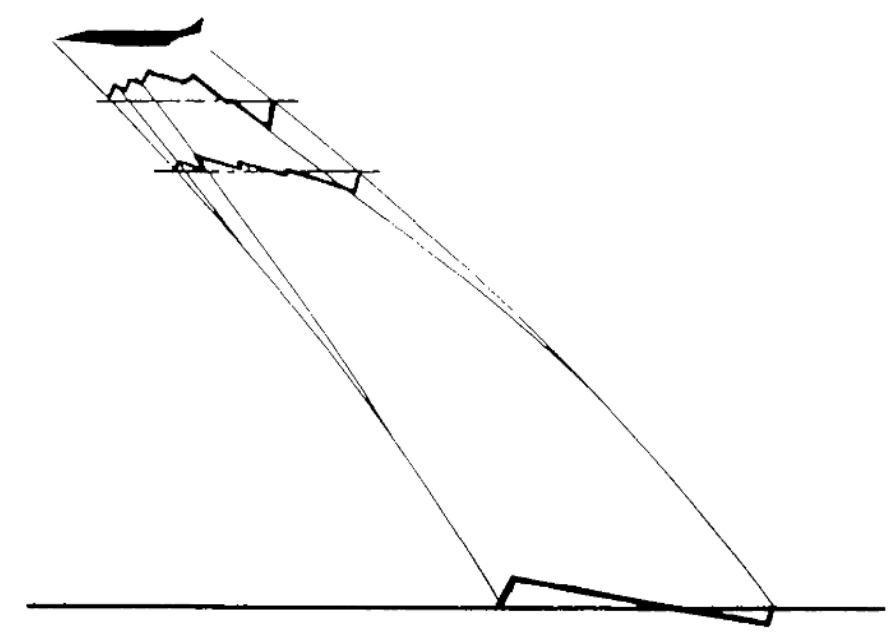

Figure 1.6: Degeneracy of the sonic-boom wave shape from a supersonic aircraft related to the long distance traveled by the same shock-wave [6].

Figure 1.6 shows the change of the sonic-boom shock-wave pressure signature generated by a supersonic aircraft with the generated noise propagating at a long distance. The non equilibrium thermodynamic condition of the high amplitude propagating wave implies that the high frequency part of the noise spectrum has a much higher acoustic dissipation rate than the lower frequency noise spectrum.

High frequency diffusive effects at non thermodynamic equilibrium due to the expansional viscosity, their modeling and computational applications have not been studied. Only some theoretical models were proposed, like the ones by Landau [20], Emanuel [21] and 
Hoover et al. [22]

\subsection{Work Background}

Computational Fluid Dynamics (CFD) have developed rapidly over the last four decades. Computing entire flow fields influenced the aerospace engineering in a positive way. New algorithms and numerical schemes were developed and created during these decades to be used from a general point of view to a more refined analysis for application. The level of physical modeling in the first days of CFD was limited to potential flows, where severe simplifications of the governing equations were made to be able to run the simulation on the available computational capacity at that time. With the quick growth of the computational capacity, the level of sophistication not only increased for the physical models but also for the numerical algorithms. Two dimensional potential flows were replaced by three dimensional simulations with non-linear governing equations. Finally methods that could calculate the underlying physics, such as compressibility and turbulence effects, could be applied to realistic cases. The industry achieved great savings both in time and in number of experimental runs needed for a given investigation.

Nowadays the dynamics levels of approximation of the computational codes used in the industry are the same ones used in the 1980's; turbulence modeling of the whole turbulence spectrum and mean valued governing equations (RANS equations). This level of sophistication did not change much over the years, a good range of simple flows can be correctly calculated this way. However, with the increase of the flow complexity that faces the aerospace industry, the need of high fidelity simulations with even smaller numerical and modeling errors imposes some serious restrictions on what numerical methods are available for solving these problems. More precise models, such as the subgrid scale modeling of the turbulent eddies (large eddy simulation), or even the simulations of all the turbulent scales (direct numerical simulation) are not yet feasible for large scale deployment in the aerospace industry. Their prohibitive computational cost makes them impractical, being restricted to academia, or government.

CFD codes application has been the same for over ten years. Nowadays, available CFD technology used in industry cannot describe with sufficient capability important phenom- 


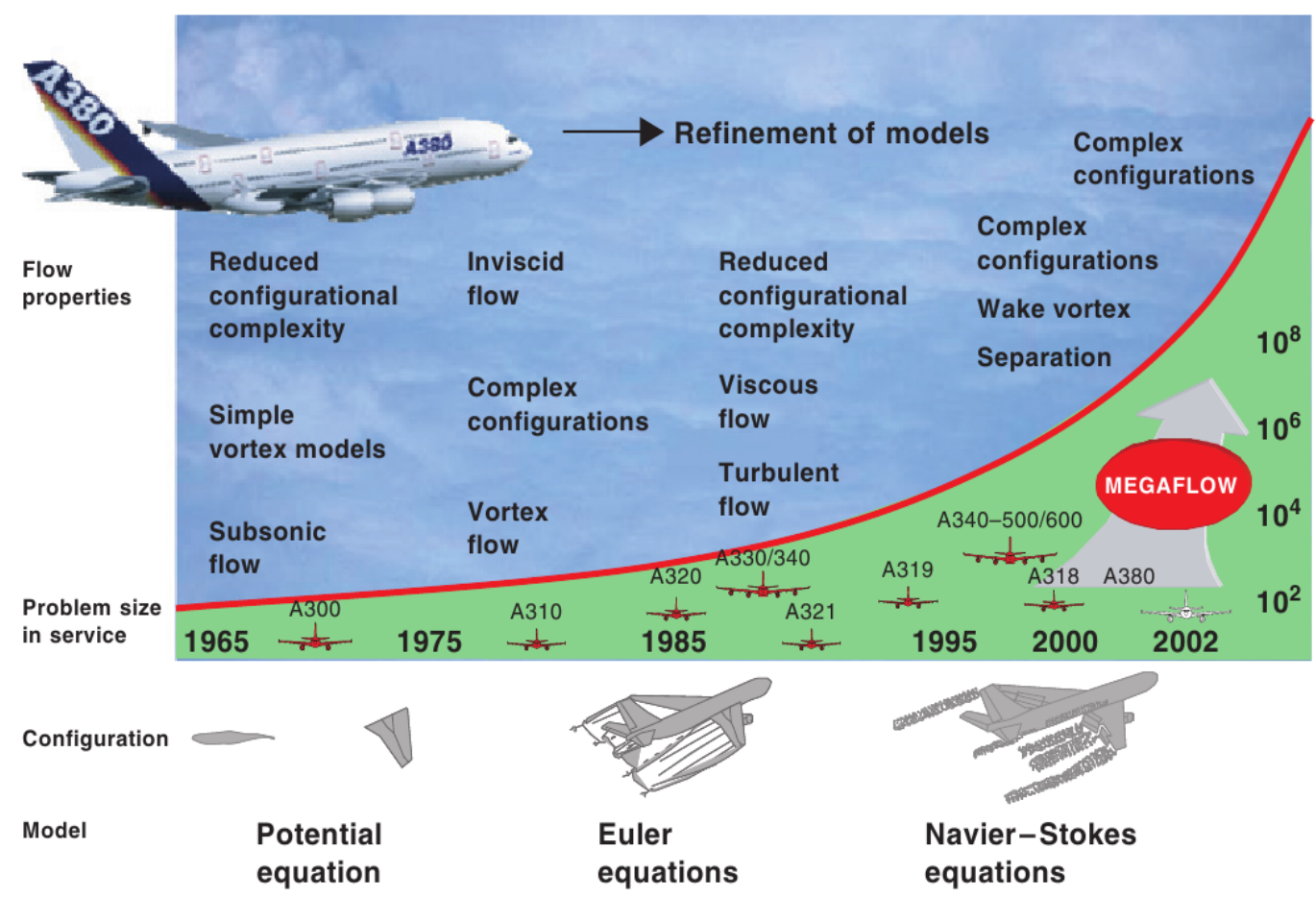

Figure 1.7: Graphic showing the capabilities and capacity of computational fluid dynamics $[23]$.

ena [24]: flow detachment, complex turbulence transition, turbomachinery flow (moving elements), mixing of different species of fluids, flows with combustion and other chemically reacting flows, noise generated from several vehicle parts, highly compressible complex flows, among others. There is a need for development of new physical models and computational algorithms to achieve a satisfactory description of these kinds of solutions both in space and in time, as can be seen in figure 1.7.

The desirable characteristics of a computational code that is able to compute a solution with the desired requirements and that provides the needed physical insight are the following [24]:

- The solution of the CFD code must rely on modeling and physical aspects only.

- Must include error and uncertainties management.

- Expected level of automatic features in all usage levels.

- Be able to use in an efficient way the available HPC (High Performance Computing) architectures. 
- Must be flexible enough to be used both in large scale applications (capacity) and in high computational demanding cases (capability).

- Must be able to work with other applications type, including CAD, CAM and finite element structural solvers.

\subsubsection{Computational Fluid Dynamics Applied to Aeracoustics}

Composed of an extensive array of physical phenomena, if the physical modeling aims for a complete description of the noise generation, propagation, and radiation mechanisms with good precision, high computational costs will be highly demanding even for efficient computational algorithms. The act of separating the problem into smaller pieces and the use of linearized (simplified) sets of governing equations have been the main choices. Regarding more complex aeroacoustic problems, the adaptation and use of traditional CFD codes have been the most common way to numerically predict the generated noise of a given case. The natural limits of these traditional codes, in terms of their numerical methods and algorithm implementation make their use impractical for more complex cases.

New computational algorithms have been developed in the last years to bridge the gaps where traditional CFD methods do not have the needed precision to describe the underlying aeroacoustic physical processes correctly. The development of these new algorithms and numerical methods has created a new branch of the computational fluid dynamics: computational aeroacoustics (CAA). The new CAA methods try to describe phenomena with high temporal gradients, where wave propagation is the main issue to be solved. Some CAA methods must have the following characteristics to be able to describe correctly the needed aeroacoustic and aerodynamic phenomena [25]:

- Must describe with a minimum of precision the largest possible range of frequencies. The calculation of waves with the highest frequency or wavenumber is one of the main objectives of computational aeroacoustics.

- The acoustic wave amplitudes are very low when compared with the aerodynamics content. A CAA numerical scheme must be able to predict these acoustic wave amplitudes with a minimum level of numerical noise. 
- Aeroacoustic problems concerns the noise calculation at long distances from the noise source. The solution must be valid from the noise source up to the radiated position, usually multiple wavelengths. CAA numerical schemes must have minimum numerical dissipation and diffusion to do so; they must be able to calculate the wave propagation and radiation at the correct physical speed regardless the computational grid orientation and with a minimum distortion of the wave content.

- Due to computational grid limitation available to describe an aeroacoustic numerical solution, the acoustic waves may present undesirable behavior at the mesh outer limits. Acoustic waves do not decay like, for example, like hydrodynamic ones, since they show reflections at the mesh boundaries. Numeric boundary conditions or filtering strategies must be used to avoid waves reflecting at these boundaries and traveling back to the source region, contaminating the computed solution.

- Aeroacoustic phenomena are typically multiscale problems. The characteristic length of an acoustic source may differ in several orders of magnitude from the characteristic wavelength. CAA methods must be able to deal with all the flow scales that may appear in the computational domain.

Besides the numerical schemes development that is necessary to solve aeroacoustic problems, new types of numerical analyses are needed to assess the numerical error. An error assessment methodology is needed to predict at a certain confidence bound the numerical error of a given numerical solution. Numerical stability is also an issue that also must be addressed, which is not only necessary to stabilize the numerical scheme, it cannot have a major influence on the numerical precision and therefore, the numerical solution.

\subsubsection{Computational Efficiency}

The use of new architectures of HPC in CFD and CAA have been a growing subject of discussions. According to Moore's Law [26], that tells that indirectly the computing capacity from CPU's doubles every two years, enabling a continuous evolution of the processing power available, and consequently, reducing the time needed for numerical simulations. Wheter this law is realistic or not, the computational capacity available from new HPC ar- 
chitectures has established a new milestone in numerical simulation capacity and capability. The increased use of Beowulf Clusters supercomputers type, which are several computers (nodes) linked together in a high-speed network and with distributed memory, makes available to any research group access to large computational power regardless of their size, by using only commodity computer parts. Another remarkable factor is on the software side; the development of libraries and language extensions that makes certain types of computing graphics units (GPU), like CUDA C/C ++ [27], Open-CL [28], Open-ACC [29], CUDA Fortran [30], Matlab [31] and co-processors [32] able to perform heavy numerical computations. The use of alternative computational architectures to perform numerical computations in the last few years allowed a significant rise in computational power, sometimes hundreds times of speedup when compared with traditional CPU serial executions.

\section{ACCELERATORS/CO-PROCESSORS}

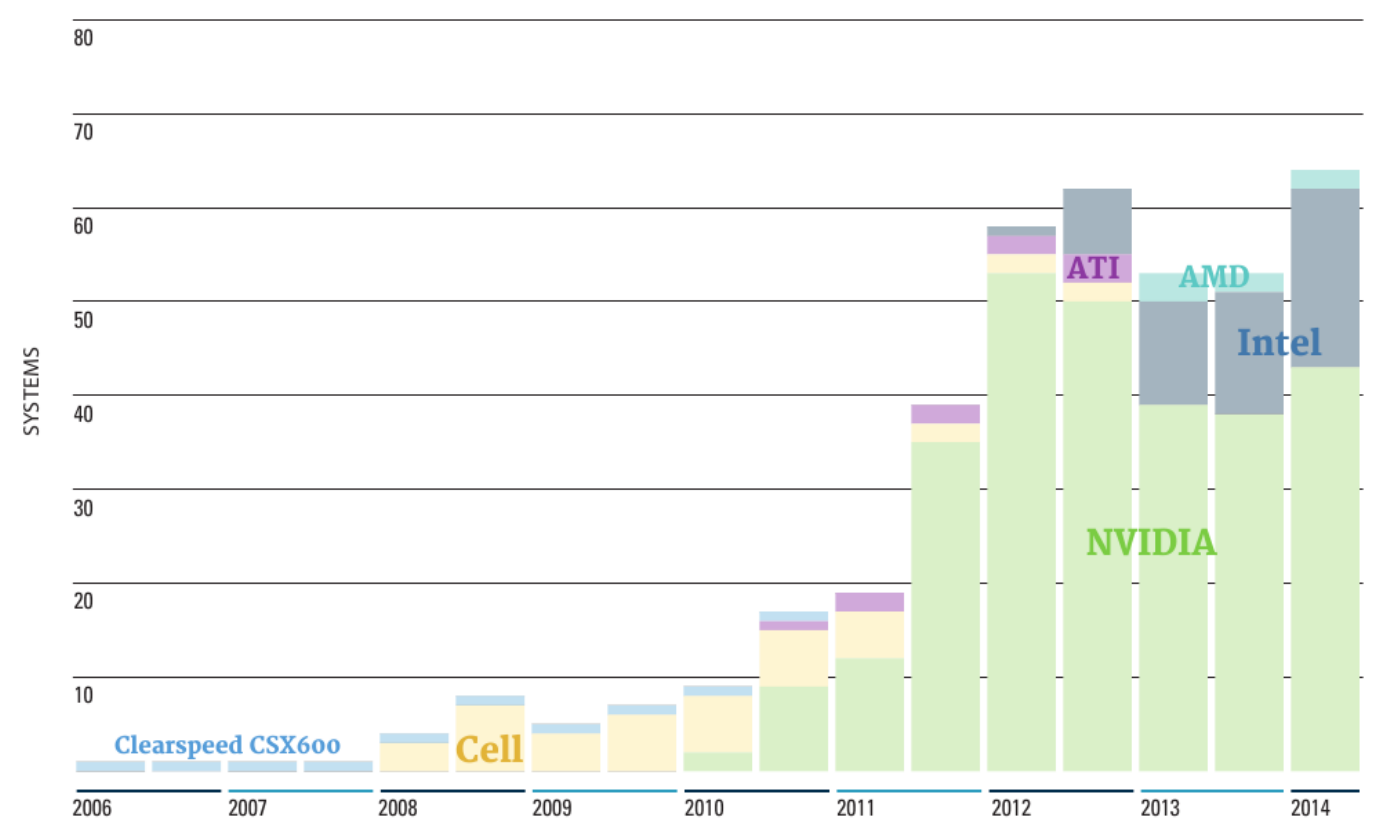

Figure 1.8: Use of GPUS and co-processors on the world's largest supercomputers [37].

Unfortunately in spite of the high speedup, results have not yet been achieved in CAA/CFD codes implemented in new HPC architectures. The type of numerical algorithm and the level of usage of the hardware will ultimately provide the implementation success and viability of the numerical code for a given HPC architecture. The actual trend of HPC shows that massively parallel codes are the ones that take full advantage of the actual cluster supercomputer's architecture. Computational codes that use implicit methods, 
and that depend upon solving of linear systems of equations, such as the ones widely used in industry: Fluent [33], CFX [34] and CFD ++ [35], rely on a great part of their execution algorithms on purely serial computations. Their numerical parallel implementation is based on older computer architectures, where the computer nodes of a cluster had only one CPU core, making the calculation purely memory distributed, where their node to node communication is done by message passing interface (MPI) libraries. These aerodynamic codes are mostly used for calculation of permanent flowfield solutions, while new computational codes takes advantage of parallel strategies from multicore CPUs and use fully explicit temporal calculations are being developed, such as PowerFLOW [36].

\section{ARCHITECTURES}

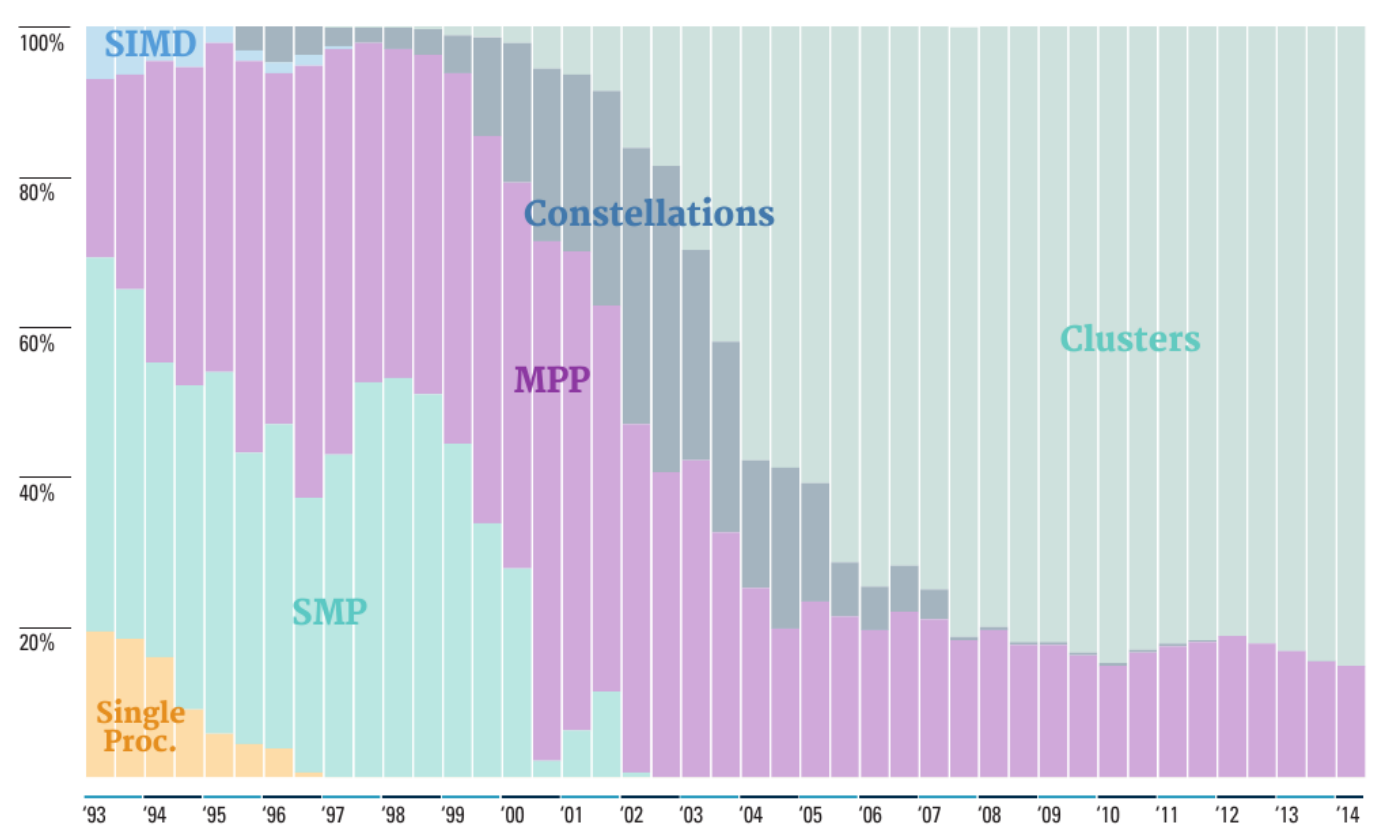

Figure 1.9: Proportion of HPC architectures used in the world's largest supercomputers [37].

With the availability of multicore CPUs, the necessity of shared memory language extensions to make full use of these type of hardware arised. For use in Beowulf cluster mainly at the academic level, there was the development of hybrid computational codes in regard to the parallel levels: at thread levels of execution for the shared memory parallel level and at distributed memory level processes distributed over the cluster nodes. The use of GPUs and co-processors goes along with the shared memory parallel execution level and is totally compatible with distributed memory parallel levels of execution. As a consequence of that 
and according to the trends shown in figures 1.8 and 1.9, the types of algorithms that will prevail in such computational environments are the massively parallel ones, where most of the execution time is spent on the numerical calculation per se with minimum time as possible with communication between the distributed memory processes.

\subsubsection{State of the Art of Prediction on Rotor and Stator Interac- tion Generated Noise}

Huge efforts have been made by industry and academia through the last years to develop numerical tools for prediction of noise generated by aeronautic turbofans. The codes of choice had been the ones used traditionally for aerodynamic calculation, where the main type of solutions of these codes are the resulting mean flows. The development of numerical methodologies that calculate the fluctuations (acoustic field) associated with the given mean flow are currently the main road followed.

The main path followed to noise prediction has been primarily on subsonic flow regimes where these types of flows may be regarded as linear, if the flow characteristic Mach number indicates such linearity. On most rotor and stator interaction noise calculation methodologies, the aerodynamic field around the rotor blades is calculated and then the outlet flow results are directly imposed as inlet flow conditions on the stator vanes region, where the instantaneous response is simulated through the use of sliding meshes or where the results are later imposed unilaterally on the stator mesh. In some cases the governing equations are not even the same for the rotor blades and stator vanes domains.

In subsonic flow calculations for the rotor blades and stator vanes interaction in axial turbomachinery, the use of traditional methods for CFD have been the primarily followed: mean permanent flows using RANS equations [38] [39] [40] [41], the use of non-permanent flows with URANS equations [42] [43] [44] [45], and yet LES simulations [46]. The linearized Euler equations are typically used when the rotor outlet flow field is unilaterally imposed on the stator vanes domain [40] [47]. The perturbations that are calculated by the rotor and stator interaction are then propagated by using linear governing equations, like the linearized Euler equations [41] [42] or the Pridmore-Brown equations [49] for sheared flows. The use of these equations has reduced computational requirements. Different discretization 
methods have been used for noise propagation in ducts when the linearized Euler equations are used, like the boundary element methods [48], the finite element methods [42] [49] [39], finite volumes method [50] and yet finite difference methods [41]. Analytical methods may be used for the noise generation and propagation in ducts like the use of Green's functions [42]. For the calculation the radiated noise from the nacelle lips, analytic methods may be used when simplified conditions are implied [51], or by the use of integration of the flow field by using acoustic analogies, like the use of the Ffowcs-Williams equation [52] [39] [44] $[53]$.

Very little attention has been given for noise prediction where the rotor blade tip speed is transonic, only a few theoretical studies are available [54].

Attention has also been given in a lesser extent to more realistic flows, where the rotor blade tip speed is supersonic. Several methodologies were developed to predict the noise generated and propagated by supersonic rotor blades. CFD has been the main methodology used to predict the buzz-saw noise produced by rotor blades with geometric non-conformity, both in two and three dimension flows [55] [56]. Other methods not based on CFD were proposed in the time domain [57] [58], and also in the frequency domain [59] [60]. For the calculation of the radiated buzz-saw noise frequency domain linearized governing equation

are commonly used, where the buzz-saw noise is treated as a classical linear wave [56], where the governing equation in this case is the Helmholtz equation with convective effects.

\subsection{Work Scope}

Most of the noise prediction methodologies previously presented in section 1.6.3 have some limitations concerning their numerical methodology or the model itself. Some tonal noise predictions simulate the interaction noise unilaterally, where the rotor influence is imposed as the inlet flow field on the stator domain, and where linearized governing equations are used. The use of linearized equations and the way that the interaction is calculated imposes severe restrictions on the allowed flow regimes. For higher Mach numbers (based on the rotor blades velocity) the non-linearity directly related with compressibility effects are not taken into account when linear governing equations are used. Realistic flows where shock-waves are the main phenomenon of the fluid flow were not considered for the rotor 
and stator interaction case. In some methods for the prediction of rotor and stator interaction noise, sliding meshes are used for the rotor blades movement and cyclic conditions are applied to emulate a complete assembly of the rotor blades and stator vanes sets. Some issues of the interpolation schemes used for the calculation of the interfaces between the meshes impose limitations when applied to flows with steep gradients, like the ones where there are shock-waves in the sliding mesh region. Considering all that was discussed above, it is important to consider a numerical methodology where the effects of mesh interface are negligible and that calculates in the most natural way the rotor blades and stator vanes interaction noise, preferably with the same governing equations in the interaction region and in any desired flow regime.

When dealing with modern aeronautic turbofans, the frequency range and propagating distances may reach high values. The thermodynamic equilibrium condition considered in the traditional governing equations for these types of flows may be doubtful and therefore the study of cases with thermodynamic non-equilibrium are proposed with the use of an already existent expansional viscosity model.

The proposed methodology of this work is about all that was discussed previously. Two main research lines will be followed; a numerical methodology that predicts the generation and propagation noise from rotor blades and stator vanes interaction by using the moving immersed boundary method, and the study and development of an existing expansional viscosity model to be applied in computational fluid dynamics cases with high frequency phenomena.

The main objectives in this work follow:

1. Develop a fully compressible numerical model based on the moving immersed boundary method that is able to predict the rotor and stator interaction noise in several compressibility flow regimes.

2. Implement and optimize the moving immersed boundary method in CUDA architecture.

3. Study the behavior of the expansional viscosity model and to adapt it to be used in computational fluid dynamics methods. 
4. Develop a numerical methodology for a first numerical study of the expansional viscosity model.

5. Validate the numerical prediction methodology of rotor and stator noise interaction for specific compressibility flow regimes and study the the behavior of the expansional viscosity under non-linear conditions through the immersed boundary method.

Concerning the rotor and stator interaction cases, an analytical theory is developed that describes the noise generation behavior from flow regimes spanning subsonic, transonic and supersonic rotor velocities. Next, propagated with the thin duct approximation, using the classical wave propagation theory and the non-linear finite shock-waves amplitude decay. This defines the tonal noise interaction modes characteristics. The results of this analysis will be used for the validation of the obtained numerical results.

On the expansional viscosity modeling, a stability analysis is done to establish the behavior of the model in linearized flows, then a first application in CFD is made so it can later be applied in more realistic aeroacoustic cases.

This work is divided in the following chapters:

- Analytical relations for noise propagation in ducts.

- The expansional viscosity model.

- Numerical methodologies.

- Numerical Results.

- Conclusions and future work.

Chapter 2 is about the mathematical simplifications applied to the governing equations, where according to the flow and boundary conditions, it is possible to obtain analytical solutions to the propagation problem compared with the numerical solutions from the rotor and stator interaction cases. For the rotor and stator interaction noise generated and propagated in subsonic and transonic rotor velocities flow regimes, the wave equation with convection effects and its solution in cylindrical coordinates is obtained. The tonal noise sources characteristics are assumed and applied along with the classical wave equation 
solution for thin annular ducts cases. In the rotor and stator interaction cases with rotor supersonic speeds, a mathematical relation for the shock-waves amplitude decay in the axial direction in the inlet direction is used and an algorithm that describes the pressure field for the buzz-saw noise case is proposed.

Chapter 3 presents the expansional viscosity model at a non-Stokesian condition, where after the linearization of the governing equation the stability analysis is made. The damping coefficient of the wave amplitude is assessed for a wide range of wavenumbers as a preliminary result for the numerical results.

Chapter 4 presents the discretization process based on the finite volume method that is applied on the moving immersed boundary method along with the Euler governing equations in the compressible form. An error analysis associated with the discretization process is made to assess its effects on the wave propagation phenomenon, at reference values of the human hearing upper threshold frequency. A modification of the already existing numerical viscosity is proposed to make the moving immersed boundary method even more stable. The non-penetrating and slipping boundary conditions are then obtained to be applied in the immersed boundary method for moving and static body volumes. A high formal and spectral order of precision for the space and time numerical scheme is used for the discretization of the governing equations used where the expansional viscosity model is applied. A high order wavenumber filter is used in this numerical scheme for the removal of the poorly resolved frequencies of the numerical solution.

On chapter 5 the validation of the proposed numerical schemes is presented and compared to the results obtained from the analytical solution. The modal response and the shock-waves behavior in the annular duct, predicted by the analytical theory, is compared with the results from the rotor and stator interaction cases. The numerical scheme proposed for the first study of the expansional viscosity is for a one dimensional wave propagation case in non-linear regime, where the effects of its physical parameters are assessed. A first implementation and testing of the expansional viscosity is made with the moving immersed boundary method for some already validated rotor and stator interaction cases.

Chapter 6 presents the conclusions regarding the results of this work, and ideas for future research are thoroughly discussed. 


\section{Chapter 2}

\section{Fundamentals of Noise Generation and Propagation in Ducts}

This chapter describes the analytical theory of noise generation and propagation in thin annular ducts. Such theory is developed from the fluid mechanics governing equations; the Navier-Stokes equations in the compressible form.

Mathematical and physical simplifications are made on the governing equations according to the type of mean flow in annular ducts. It is considered a priori that the flow regime is subsonic, linear and isentropic. From this consideration the simplifications made on the governing equations and by the type of mean flow, a general analytic solution is obtained. In this solution certain types of fundamental waves may be identified, called acoustic modes. The implicit boundary conditions of the angular coordinates, the type of walls considered for the radial coordinate and the imposed mean flow dictates the shape and propagating conditions of these modes and how they compose the solution of the noise at a certain frequency.

Other assumptions are made to characterize the source of noise induced by the rotor and stator interaction in subsonic flow conditions. It is not possible to fully predict the source of noise, but making some assumptions it is possible to describe in a way the modal behavior of this interaction. The simplification of thin annular ducts is used to restrict the modal set from the noise source, making it possible to predict all the generated modes and their consequence in the propagation theory. 
A theory of the noise generation and propagation with the rotor at supersonic speeds, where the flow characteristics are different from the ones made for the subsonic and transonic rotor flow regimes, is developed. A one dimensional and transient solution of the decay of a propagating shock-wave though a moving media is used on the thin annular duct with axial subsonic uniform flow. Considering a system of coordinates moving with a base shock-wave, the decay and interaction of the system of supersonic rotor generated shock-waves can be totally predicted through the duct.

Two types of noise sources conditions are used for the rotor alone at supersonic speeds: the ideal and the realistic. In the ideal condition the rotor to rotor shock-waves are identical in intensity and propagation angles. For the realistic rotor conditions the system of shockwaves is not homogeneous. Small geometric differences between the rotor blades makes each shock-wave have its own propagating angle and intensity, resulting in different shockwave to shock-wave decay and propagation velocities and thus, occurring shock coalescence depending on the flow and geometric conditions. A methodology that predicts these shockwaves propagation, decay and coalescence through the duct is developed with ideas already available on the literature.

With the analytical solutions developed in this chapter for all the flow regimes of a turbofan engine: subsonic, transonic and supersonic, it is possible to establishment a base of analytical theory for comparison with the numerical results of the rotor and stator interaction noise, and thus, validating one of the numeric methodologies proposed in this work.

\subsection{Governing Equations}

Three physical principles are used to represent the generation and propagation of noise in the rotor and stator interaction:

- Mass conservation (or continuity): the system mass is conserved.

- Newton's second law: the resulting forces acting on a body result in a change of momentum on that body. 
- First law of thermodynamics: the change of internal energy of a given system is due to macroscopic (work) and microscopic (heat transfer) phenomena.

The fluid is considered continuous, i.e., the characteristic length scales are large enough that a given material point of the flow has enough molecules to not have any property fluctuations related to molecular dynamics. The changes of these properties at a given material point are due to the flow dynamics only; the individual fluctuations of molecular orders of magnitude are not considered here.

The mathematical derivation of the physical laws described above are be obtained starting from the Reynolds transport theorem. For a given generic quantity $f$, where $f$ may be a scalar, vector or tensor quantity, such quantity is conserved in a control volume $V$ with boundary $S$ from the given relation [61]:

$$
\frac{d}{d t} \int_{V(t)} \mathbf{f} d V=\int_{V(t)} \frac{\partial \mathbf{f}}{\partial t} d V+\oint_{S(t)}(\mathbf{u} \cdot \mathbf{n}) \mathbf{f} d S,
$$

where $\mathbf{u}$ is the velocity vector, $\mathbf{n}$ represents the surface normal vector at an infinitesimal surface $d S$. The vector direction convention used here is that the positive value is directed outwards from the surface $S$.

The idea behind the Reynolds transport theorem is to establish an integral relation between any conserved quantity of a control volume, where the flux of this quantity is accounted for in the surface as well as its transformation by a given phenomenon-inside this control volume. By applying the Reynolds transport theorem on the physical laws described above, it is possible to obtain the integral equations that mathematically describe these physical laws on a given control volume fixed in time:

- Conservation of mass:

$$
\int_{V} \frac{\partial \rho}{\partial t} d V+\oint_{S} \rho \mathbf{u} \cdot \mathbf{d} \mathbf{S}=0
$$

- Newton's second law:

$$
\int_{V} \frac{\partial}{\partial t}(\rho \mathbf{u}) d V+\oint_{S} \rho \mathbf{u}(\mathbf{u} \cdot \mathbf{d} \mathbf{S})=-\oint_{S} p \cdot \mathbf{d} \mathbf{S}+\oint_{S} \tau \cdot \mathbf{d} \mathbf{S}+\int_{V} \rho \mathbf{f}_{e} d V
$$


- First law of thermodynamics:

$$
\begin{aligned}
\int_{V} \frac{\partial}{\partial t}\left(\rho e_{t}\right) d V+\oint_{S} \rho e_{t} \mathbf{u} \cdot \mathbf{d} \mathbf{S}= \\
\quad-\oint_{S} \mathbf{q} \cdot \mathbf{d} \mathbf{S}+\int_{V}\left(\rho \mathbf{f}_{e} \cdot \mathbf{u}\right) d V+\oint_{S}[(-p \mathbf{I}+\tau) \cdot \mathbf{u}] \cdot \mathbf{d} \mathbf{S},
\end{aligned}
$$

where $\rho$ is the fluid density, $p$ is the fluid pressure, $\tau$ is the shear stress tensor, $\mathbf{f}_{\mathbf{e}}$ are the external body forces applied on the fluid, $e_{t}$ is the fluid total energy, $\mathbf{q}$ is the heat conduction vector, $T$ is the fluid temperature and $I$ is the identity tensor.

By applying the divergence theorem in the previous set of equations, the surface integrals are transformed into volume integrals. One can now put all the integrating terms in a single volume integral.

- Mass conservation:

$$
\int_{V}\left(\frac{\partial \rho}{\partial t}+\nabla \cdot(\rho \mathbf{u})\right) d V=0
$$

- Newton's second law:

$$
\int_{V}\left(\frac{\partial}{\partial t}(\rho \mathbf{u})+\nabla \cdot(\rho \mathbf{u} \otimes \mathbf{u}+p \mathbf{I}-\tau)-f_{e}\right) d V=0 .
$$

- First law of thermodynamics:

$$
\int_{V}\left(\frac{\partial}{\partial t}\left(\rho e_{t}\right)+\nabla \cdot\left(\rho \mathbf{u} e_{t}+\mathbf{q}-\tau \cdot \mathbf{u}+p \mathbf{I} \cdot \mathbf{u}\right)-f_{e} \cdot \mathbf{u}\right)=0
$$

where $\otimes$ denotes the tensor product of the velocity vector with itself.

This integral form of the governing equations may be simplified using the localization theorem, which states that if a volume integral equals zero for any integration volume, then the arguments are zero. As a consequence, we have the following form of the governing equations:

- Mass conservation:

$$
\frac{\partial \rho}{\partial t}+\nabla \cdot(\rho \mathbf{u})=0
$$

- Newton's second law:

$$
\frac{\partial}{\partial t}(\rho \mathbf{u})+\nabla \cdot(\rho \mathbf{u} \otimes \mathbf{u})=-\nabla \cdot(p \mathbf{I})+\nabla \cdot \tau+f_{e}
$$


- First law of thermodynamics:

$$
\frac{\partial}{\partial t}\left(\rho e_{t}\right)+\nabla \cdot\left(\rho \mathbf{u} e_{t}\right)=\nabla \cdot(-\mathbf{q}+\tau \cdot \mathbf{u}-p \mathbf{I} \cdot \mathbf{u})+f_{e} \cdot \mathbf{u}
$$

Equations 2.8 to 2.10 show the governing equations in the differential conservative form. The conservative form is evident in the way convective terms are grouped together under the divergent operator. The use of governing equations under the conservative form is more convenient for numeric applications, where in their discretization process numerical sources do not appear [23].

The fluid used here is air; compressible and calorically perfect, Newtonian viscous and Stokesian, if not stated otherwise. The deviatoric part of the stress tensor is Newtonian and compressible:

$$
\tau=\mu\left[\left(\nabla \mathbf{u}+(\nabla \mathbf{u})^{T}\right)-\frac{2}{3}(\nabla \cdot \mathbf{u}) \mathbf{I}\right],
$$

where $\mu$ is the dynamic viscosity coefficient.

Fourier's law of heat conductivity is applied with a constant conductivity coefficient:

$$
\mathbf{q}=-k \nabla T
$$

The closure constitutive equations are the ideal gas law equation:

$$
p=\rho \mathcal{R} T,
$$

where $\mathcal{R}$ is the ideal gas constant. Sutherland's viscosity law is also used:

$$
\mu=\mu_{0} \frac{T_{0}+C}{T+C}\left(\frac{T}{T_{0}}\right)^{3 / 2},
$$

where the constants for the air are the following: $C=120 K, T_{0}=291.15 K$ e $\mu_{0}=$ $18.27 \cdot 10^{-6} \mathrm{~Pa} \cdot \mathrm{s}$. The thermodynamic relation for the internal energy used here is:

$$
e=c_{v} T
$$

where $c_{v}$ is the specific heat at constant volume. 


\subsection{Noise Generation and Propagation in Ducts with Subsonic Noise Sources}

One of the main parts of the modern aeronautic turbofans is the duct in which the turbofan is enclosed. The duct inner wall is the hub and the outer wall is the nacelle. The duct can be modeled as circular (tipically inlet) or annular (tipically outlet) and this choice affects the propagation. The noise may propagate in upstream and downstream directions, where the flow conditions may affect the propagation. As a consequence, that defines the mathematical behavior of the solution concerning these propagating waves that will be compared with the numeric solutions. The mathematical conditions found in the propagating region may be regarded as linear and the classical wave theory may be used to define the flow field.

\subsubsection{Wave Equation with Convective Effects Derivation}

In this section the linear acoustic phenomena equation is related to a mean subsonic flow is derived. The first few assumptions are related to the diffusivity effects, such as momentum diffusion and heat conduction, which are neglected in the propagation mechanics $(\tau=\mathbf{0}$, $\mathbf{q}=0$ ), as well as the body forces like gravity are also neglected here. We then obtain some simplifications for the governing equations:

- Mass conservation:

$$
\frac{\partial \rho}{\partial t}+\nabla \cdot(\rho \mathbf{u})=0
$$

- Newton's second law:

$$
\frac{\partial}{\partial t}(\rho \mathbf{u})+\nabla \cdot(\rho \mathbf{u} \otimes \mathbf{u})=-\nabla \cdot(p \mathbf{I})
$$

- First law of thermodynamics:

$$
\frac{\partial}{\partial t}\left(\rho e_{t}\right)+\nabla \cdot\left(\rho \mathbf{u} e_{t}\right)=-\nabla \cdot(p \mathbf{I} \cdot \mathbf{u}) .
$$

Taking the first term from the left hand side of equation 2.18 and applying the product rule for derivatives:

$$
\frac{\partial}{\partial t}\left(\rho e_{t}\right)=\rho \frac{\partial e_{t}}{\partial t}+e_{t} \frac{\partial \rho}{\partial t},
$$


applying the following vector identity on the second term:

$$
\nabla \cdot\left(\rho e_{t} \mathbf{u}\right)=e_{t} \nabla \cdot(\rho \mathbf{u})+\rho \mathbf{u} \cdot \nabla e_{t}
$$

Equation 2.18 is then rewritten as:

$$
\frac{\partial}{\partial t}\left(\rho e_{t}\right)-e_{t}\left(\frac{\partial \rho}{\partial t}+\nabla \cdot(\rho \mathbf{u})\right)+\nabla \cdot\left(\rho e_{t} \mathbf{u}\right)=\rho \frac{\partial e}{\partial t}+\rho \mathbf{u} \cdot \nabla e_{t}
$$

The term in parenthesis on the right hand side of equation 2.21 is equal zero according to continuity equation 2.16 :

$$
\frac{\partial}{\partial t}\left(\rho e_{t}\right)+\nabla \cdot\left(\rho e_{t} \mathbf{u}\right)=\rho \frac{\partial e}{\partial t}+\rho \mathbf{u} \cdot \nabla e_{t}
$$

The material derivative is defined as:

$$
\frac{D}{D t} \equiv \frac{\partial}{\partial t}+\mathbf{u} \cdot \nabla
$$

and represents the variation of the applied quantity related to the transport of it. The first term represents the local change of this quantity, the second term represents the change of this quantity that is moving with the flow. As a consequence:

$$
\rho \frac{D e_{t}}{D t}=\frac{\partial \rho e_{t}}{\partial t}+\nabla \cdot\left(\rho \mathbf{u} e_{t}\right)
$$

By applying the material derivative definition on the Newton's second law equation 2.17 we have:

$$
\rho \frac{D \mathbf{u}}{D t}=\frac{\partial \rho \mathbf{u}}{\partial t}+\nabla \cdot(\rho \mathbf{u} \otimes \mathbf{u})
$$

In the continuity equation, the transformation of the conservative form into the nonconservative is simpler. Taking the vector identity 2.20 and taking the second term from equation 2.16 we have:

$$
\nabla \cdot(\rho \mathbf{u})=\rho \nabla \cdot \mathbf{u}+\mathbf{u} \cdot \nabla \rho
$$

we arrive at the following form:

$$
\frac{\partial \rho}{\partial t}+\mathbf{u} \cdot \nabla \rho+\rho \nabla \cdot \mathbf{u}=0
$$

With the material derivative definition being applied into equation 2.27, we can obtain the other governing equations into the non-conservative form: 
- Mass conservation:

$$
\frac{D \rho}{D t}=-\rho \nabla \cdot \mathbf{u}
$$

- Newton's second law:

$$
\rho \frac{D \mathbf{u}}{D t}=-\nabla \cdot p \mathbf{I}
$$

- First law of thermodynamics:

$$
\rho \frac{D e_{t}}{D t}=-\nabla \cdot(p \mathbf{I} \cdot \mathbf{u})
$$

The first law of thermodynamics equation does not present a convenient form for the linearization process that will be applied later on. Using the total energy definition, the following equation is obtained:

$$
e_{t}=e+\frac{1}{2} \mathbf{u} \cdot \mathbf{u}
$$

where the first term of the right hand side is the fluid internal energy and the second term is the fluid kinetic energy. The internal energy represents a purely thermodynamic property, whereas the kinetic energy represents a relation in common for both the kinematic and thermodynamic fields.

Introducing now the index notation, that implies the summation convention of the terms with repeated indexes, thus simplifying the mathematical notation. Now by applying the summation convention into equation 2.30 and combining it with equation 2.31:

$$
\rho \frac{D}{D t}\left(e+\frac{1}{2} u_{i} u_{j} \delta_{i j}\right)=\rho \frac{D}{D t}\left(e+\frac{1}{2} u_{i} u_{i}\right)=-\frac{\partial}{\partial x_{i}}\left(p \delta_{i j} u_{k} \delta_{j k}\right)=-\frac{\partial}{\partial x_{i}}\left(p u_{i}\right),
$$

where $\delta_{i j}$ is the Kronecker's delta function:

$$
\delta_{i j}=\left\{\begin{array}{l}
1 \text { if } i=j, \\
0 \text { if } i \neq j .
\end{array}\right.
$$

Expanding the terms from both sides of equation 2.32:

$$
\rho\left(\frac{D e}{D t}+\frac{1}{2}\left(u_{i} \frac{D u_{i}}{D t}+u_{i} \frac{D u_{i}}{D t}\right)\right)=\rho\left(\frac{D e}{D t}+u_{i} \frac{D u_{i}}{D t}\right)=-u_{i} \frac{\partial p}{\partial x_{i}}-p \frac{\partial u_{i}}{\partial x_{i}} .
$$

Rearranging equation 2.29 where the material derivative is the only term on the left hand side yields:

$$
\frac{D u_{i}}{D t}=-\frac{1}{\rho} \frac{\partial p}{\partial x_{i}}
$$


and now substituting the above equation in the left hand side of equation 2.34, we have:

$$
\rho\left(\frac{D e}{D t}-\frac{u_{i}}{\rho} \frac{\partial p}{\partial x_{i}}\right)=-u_{i} \frac{\partial p}{\partial x_{i}}-p \frac{\partial u_{i}}{\partial x_{i}}
$$

Simplifying equation 2.36 :

$$
\rho \frac{D e}{D t}=-p \frac{\partial u_{i}}{\partial x_{i}}
$$

Besides dissipation effects on momentum due to viscosity, and on the energy due to heat conduction, other phenomena such as shock-waves, that are discontinuities on the flow variables, including entropy, will all be neglected in this section. The flow is solely subsonic and at low levels of compressibility. To translate this idea mathematically, the following form of the first law of thermodynamics for an infinitesimal closed system into a reversible process is introduced:

$$
T d s=d e+p d\left(\rho^{-1}\right)
$$

where $d s$ is an infinitesimal change in entropy. Developing the second term of the right hand side of the equation above:

$$
d\left(\rho^{-1}\right)=-\frac{1}{\rho^{2}} d \rho
$$

By combining equations 2.38 and 2.39 with the material derivative definition, isolating the internal energy term and multiplying both sides by the fluid density:

$$
\rho \frac{D e}{D t}=\rho T \frac{D s}{D t}+\frac{p}{\rho} \frac{D \rho}{D t} .
$$

By combining equation 2.40 with equation 2.37:

$$
\rho T \frac{D s}{D t}+\frac{p}{\rho} \frac{D \rho}{D t}=-p \frac{\partial u_{i}}{\partial x_{i}}
$$

Rearranging the terms of equation 2.41:

$$
\rho T \frac{D s}{D t}=-p\left(\frac{1}{\rho} \frac{D \rho}{D t}+\rho \frac{\partial u_{i}}{\partial x_{i}}\right)
$$

The term in parenthesis on the right hand side from equation 2.42 is the continuity equation, i.e., equation 2.28 and as a consequence:

$$
\frac{D s}{D t}=0
$$


which implies that the entropy $s$ is constant along a streamline. Flows in which the entropy is constant through the streamline are called isentropic flows.

Equation 2.43 represents an important physical and mathematical consequence. With dissipation phenomena being neglected, such as viscous diffusion of momentum, heat conduction and nonlinear compressibility effects such as shock-waves, the energy equation is decoupled from the other governing equations and it is not dependent anymore on the other physical principles of the other equations.

A decomposition of the variables of the system of governing equations 2.28 and 2.29 based on mean values and acoustic perturbations is proposed. The following decomposition is very much like the Reynolds decomposition in turbulence, where the fluctuations of turbulent processes are smaller than their hydrodynamic permanent counterpart. This perturbation relation is applied on the system of governing equation in the following manner:

$$
f=f_{0}+f^{\prime}
$$

where the subscript ${ }_{0}$ is the reference or mean value of the fluid and the superscript ' ${ }^{\prime}$ is relative to the acoustic fluctuations of the flow for a given general variable $f$. By applying this perturbations relation in the governing equations we have:

- Mass conservation:

$$
\frac{\partial}{\partial t}\left(\rho_{0}+\rho^{\prime}\right)+\left(\mathbf{u}_{0}+\mathbf{u}^{\prime}\right) \cdot \nabla\left(\rho_{0}+\rho^{\prime}\right)+\left(\rho_{0}+\rho^{\prime}\right) \nabla \cdot\left(\mathbf{u}_{0}+\mathbf{u}^{\prime}\right)=0
$$

- Newton's second law:

$$
\left(\rho_{0}+\rho^{\prime}\right)\left(\frac{\partial}{\partial t}\left(\mathbf{u}_{0}+\mathbf{u}^{\prime}\right)+\left(\mathbf{u}_{0}+\mathbf{u}^{\prime}\right) \cdot \nabla\left(\mathbf{u}_{0}+\mathbf{u}^{\prime}\right)\right)+\nabla\left(p_{0}+p^{\prime}\right)=0
$$

Sound intensity values are assumed to satisfy the following relation:

$$
p_{0} \gg p^{\prime}
$$

Applying the perturbation relation from equation 2.44 into the continuity equation 2.28 and Newton's second law 2.29, and neglecting second order fluctuation terms in all variables according with equation 2.47 , we have: 
- Mass conservation:

$$
\frac{\partial \rho^{\prime}}{\partial t}+\mathbf{u}_{0} \cdot \nabla \rho_{0}+\mathbf{u}^{\prime} \cdot \nabla \rho_{0}+\mathbf{u}_{0} \cdot \nabla \rho^{\prime}+\rho_{0} \nabla \cdot \mathbf{u}_{0}+\rho^{\prime} \nabla \cdot \mathbf{u}_{0}+\rho_{0} \nabla \cdot \mathbf{u}^{\prime}=0 .
$$

- Newton's second law:

$$
\rho_{0} \frac{\partial \mathbf{u}^{\prime}}{\partial t}+\rho_{0} \mathbf{u}_{0} \cdot \nabla \mathbf{u}_{0}+\rho_{0} \mathbf{u}_{0} \cdot \nabla \mathbf{u}^{\prime}+\rho^{\prime} \mathbf{u}_{0} \cdot \nabla \mathbf{u}_{0}+\rho_{0} \mathbf{u}^{\prime} \cdot \nabla \mathbf{u}_{0}+\nabla p_{0}+\nabla p^{\prime}=0
$$

By applying the variables decomposition into equations 2.28 and 2.29 for a permanent flow field without fluctuations, i.e., mean flow only, we have:

- Mass conservation:

$$
\nabla \cdot\left(\rho_{0} \mathbf{u}_{0}\right)=\rho_{0} \nabla \cdot \mathbf{u}_{0}+\mathbf{u}_{0} \cdot \nabla \rho_{0}=0
$$

- Newton's second law:

$$
\rho_{0} \mathbf{u}_{0} \cdot \nabla \mathbf{u}_{0}=-\nabla p_{0}
$$

Now substituting equations 2.50 and 2.51 into their respective equations 2.48 and 2.49, we have:

- Mass conservation:

$$
\frac{\partial \rho^{\prime}}{\partial t}+\mathbf{u}^{\prime} \cdot \nabla \rho_{0}+\mathbf{u}_{0} \cdot \nabla \rho^{\prime}+\rho^{\prime} \nabla \cdot \mathbf{u}_{0}+\rho_{0} \nabla \cdot \mathbf{u}^{\prime}=0
$$

- Newton's second law:

$$
\rho_{0} \frac{\partial \mathbf{u}^{\prime}}{\partial t}+\rho_{0} \mathbf{u}_{0} \cdot \nabla \mathbf{u}^{\prime}+\rho^{\prime} \mathbf{u}_{0} \cdot \nabla \mathbf{u}_{0}+\rho_{0} \mathbf{u}^{\prime} \cdot \nabla \mathbf{u}_{0}+\nabla p^{\prime}=0
$$

The next simplification is related to the mean flow. The space and time derivatives of the mean variables are equal zero, resulting into a free stream solution for the mean flow:

- Mass conservation:

$$
\frac{\partial \rho^{\prime}}{\partial t}+\mathbf{u}_{0} \cdot \nabla \rho^{\prime}+\rho_{0} \nabla \cdot \mathbf{u}^{\prime}=0
$$

- Newton's second law:

$$
\rho_{0} \frac{\partial \mathbf{u}^{\prime}}{\partial t}+\rho_{0} \mathbf{u}_{0} \cdot \nabla \mathbf{u}^{\prime}+\nabla p^{\prime}=0
$$


Taking the derivative of equation 2.54 in time and taking the divergence of equation 2.55, we have:

- Mass conservation:

$$
\frac{\partial^{2} \rho^{\prime}}{\partial t^{2}}+\mathbf{u}_{0} \cdot \frac{\partial}{\partial t}\left(\nabla \rho^{\prime}\right)+\rho_{0} \frac{\partial}{\partial t}\left(\nabla \cdot \mathbf{u}^{\prime}\right)=0
$$

- Newton's second law:

$$
\rho_{0} \frac{\partial}{\partial t}\left(\nabla \cdot \mathbf{u}^{\prime}\right)+\rho_{0} \mathbf{u}_{0} \cdot \nabla^{2} \mathbf{u}^{\prime}+\nabla^{2} p^{\prime}=0
$$

Subtracting equation 2.57 from equation 2.56 we have:

$$
\frac{\partial^{2} \rho^{\prime}}{\partial t^{2}}+\mathbf{u}_{0} \cdot \frac{\partial}{\partial t}\left(\nabla \rho^{\prime}\right)-\rho_{0} \mathbf{u}_{0} \cdot \nabla^{2} \mathbf{u}^{\prime}-\nabla^{2} p^{\prime}=0 .
$$

From the following vector identity:

$$
\nabla \times(\nabla \times \mathbf{f})=\nabla(\nabla \cdot \mathbf{f})-\nabla \cdot(\nabla \mathbf{f})
$$

we substitute it on the third term of equation 2.58:

$$
\rho_{0} \mathbf{u}_{0} \cdot \nabla^{2} \mathbf{u}^{\prime}=\rho_{0} \mathbf{u}_{0} \cdot\left(\nabla \cdot \nabla \mathbf{u}^{\prime}\right)=\rho_{0} \mathbf{u}_{0} \cdot\left(\nabla\left(\nabla \cdot \mathbf{u}^{\prime}\right)-\nabla \times\left(\nabla \times \mathbf{u}^{\prime}\right)\right)
$$

Since the velocity fluctuation terms of the acoustic field are irrotational [63] $\left(\nabla \times \mathbf{u}^{\prime}=0\right)$, we have:

$$
\rho_{0} \mathbf{u}_{0} \cdot \nabla^{2} \mathbf{u}^{\prime}=\rho_{0} \mathbf{u}_{0} \cdot\left(\nabla\left(\nabla \cdot \mathbf{u}^{\prime}\right)\right)
$$

Now, isolating the term $\nabla \cdot \mathbf{u}^{\prime}$ on equation 2.54 and substituting it on equation 2.58 :

$$
\frac{\partial^{2} \rho^{\prime}}{\partial t^{2}}+\mathbf{u}_{0} \cdot \frac{\partial}{\partial t}\left(\nabla \rho^{\prime}\right)+\mathbf{u}_{0} \cdot \nabla\left(\frac{\partial \rho^{\prime}}{\partial t}+\mathbf{u}_{0} \cdot \nabla \rho^{\prime}\right)-\nabla^{2} p^{\prime}=0
$$

Rearranging equation 2.62 we have:

$$
\frac{\partial^{2} \rho^{\prime}}{\partial t^{2}}+2 \mathbf{u}_{0} \cdot \frac{\partial}{\partial t}\left(\nabla \rho^{\prime}\right)+\mathbf{u}_{0} \cdot \nabla\left(\mathbf{u}_{0} \cdot \nabla \rho^{\prime}\right)-\nabla^{2} p^{\prime}=0
$$

On the third term of equation 2.63, we apply the following vector relation:

$$
\nabla(\mathbf{f} \cdot \mathbf{g})=(\mathbf{f} \cdot \nabla) \mathbf{g}+(\mathbf{g} \cdot \nabla) \mathbf{f}+\mathbf{f} \times(\nabla \times \mathbf{g})+\mathbf{g} \times(\nabla \times \mathbf{f})
$$


Again, we consider that the flow is irrotational $\left(\nabla \times \mathbf{u}^{\prime}=0\right)$ and that the curl of a gradient is always equal zero $(\nabla \times(\nabla \mathbf{f})=0)$, then combining equation 2.64 with equation 2.63 we have:

$$
\frac{\partial^{2} \rho^{\prime}}{\partial t^{2}}+2 \mathbf{u}_{0} \cdot \frac{\partial}{\partial t}\left(\nabla \rho^{\prime}\right)+\mathbf{u}_{0} \cdot\left(\nabla \rho^{\prime} \cdot \nabla \mathbf{u}_{0}+\left(\mathbf{u}_{0} \cdot \nabla\right) \nabla \rho^{\prime}\right)-\nabla^{2} p^{\prime}=0 .
$$

Considering the mean flow as constant over time and space $\left(\nabla \mathbf{u}_{\mathbf{0}}=0\right)$ :

$$
\frac{\partial^{2} \rho^{\prime}}{\partial t^{2}}+2 \mathbf{u}_{0} \cdot \frac{\partial}{\partial t}\left(\nabla \rho^{\prime}\right)+\mathbf{u}_{0} \cdot\left(\mathbf{u}_{0} \cdot \nabla\right) \nabla \rho^{\prime}-\nabla^{2} p^{\prime}=0
$$

Applying the Taylor series for the pressure fluctuations as a function of density and entropy, we have:

$$
p_{0}+p^{\prime}=p\left(\rho_{0}+\rho^{\prime}, s\right)=p\left(\rho_{0}, s\right)+\left.\rho^{\prime} \frac{\partial p(\rho, s)}{\partial \rho}\right|_{0}+O\left(\rho^{\prime 2}\right),
$$

with the terms related to entropy equal zero since the flow is isentropic.

The derivative of equation 2.67 is done under the mean values of pressure and density $\left(p_{0}, \rho_{0}\right)$, which its units are in the square of velocity. We then define the velocity of propagation of the isentropic perturbations on the mean flow, or common called sound velocity:

$$
c_{0}=\sqrt{\left(\frac{\partial p}{\partial \rho}\right)_{s}}
$$

or in the more common form used for variable change of the wave equation:

$$
p^{\prime}=c_{0}^{2} \rho^{\prime}
$$

Combining equation 2.69 with equation 2.66 we may obtain the wave equation with only one variable, where it may be expressed as function of pressure or density only:

$$
\frac{1}{c_{0}^{2}} \frac{\partial^{2} p^{\prime}}{\partial t^{2}}+\frac{2 \mathbf{u}_{0}}{c_{0}^{2}} \cdot \frac{\partial}{\partial t}\left(\nabla p^{\prime}\right)+\frac{\mathbf{u}_{0}}{c_{0}^{2}} \cdot\left(\mathbf{u}_{0} \cdot \nabla\right) \nabla p^{\prime}-\nabla^{2} p^{\prime}=0
$$

Equation 2.70 is the more general form for the classical wave equation with constant mean flow over space and time.

\subsubsection{Wave Equation Solution Development}

In the following approximation for a turbofan engine duct, the coordinate system that better suits the nacelle-hub geometry is the cylindrical coordinates, where its rotation axis 


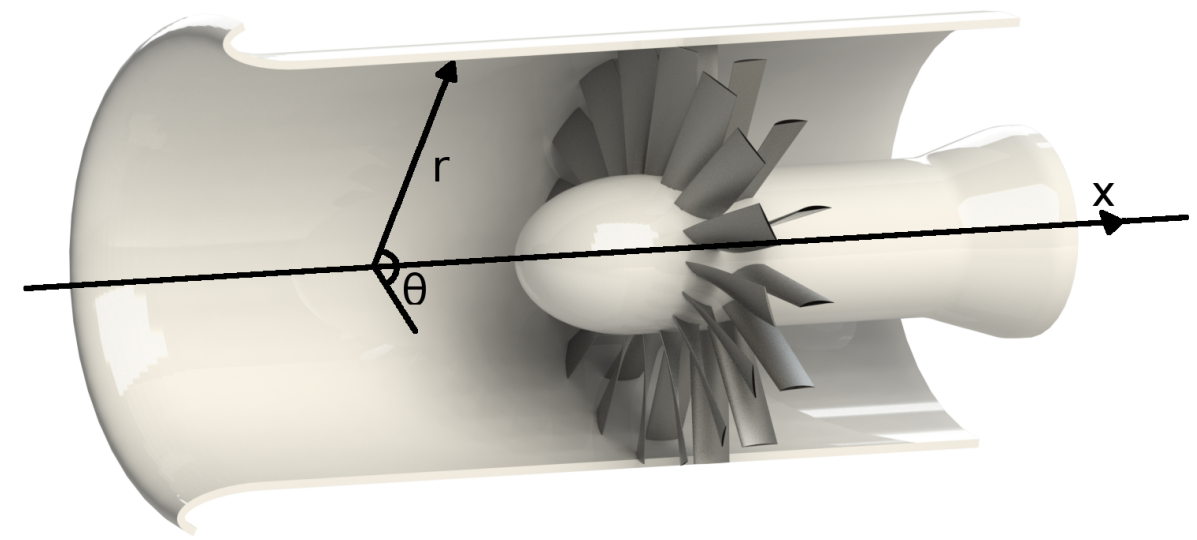

Figure 2.1: Cylindrical coordinate system used on the wave equation solution of a duct with the nacelle and the hub. CAD model of the NASA's Active Noise Control Fan rig.

is defined at the rotation axis of the rotor blades and with the constant flow parallel to the $x$ axis. For the sake of simplicity the origin of the system of coordinates is positioned at the same axial position of the rotor blades and stator vanes, also defined as a compact source on the axial direction [64]. Now defining the mean flow vector $\mathbf{u}_{0}=U \hat{\mathbf{i}}$ on equation 2.70, we may obtain the final form of the wave equation that the solution will be obtained. By analyzing this solution, conclusions about the modal behavior of the propagated noise in aeronautical turbofans may be obtained analytically at the considered flow regime:

$$
\frac{1}{c_{0}^{2}} \frac{\partial^{2} p^{\prime}}{\partial t^{2}}+\frac{2 U}{c_{0}^{2}} \frac{\partial}{\partial t}\left(\nabla p^{\prime}\right)+\frac{U^{2}}{c_{0}^{2}} \frac{\partial^{2} p^{\prime}}{\partial x^{2}}-\nabla^{2} p^{\prime}=0
$$

Reducing the first three terms of equation 2.71:

$$
\frac{1}{c_{0}^{2}}\left(\frac{\partial}{\partial t}+U \frac{\partial}{\partial x}\right)^{2} p^{\prime}-\nabla^{2} p^{\prime}=0
$$

Defining the $\nabla$ operator in cylindrical coordinates:

$$
\nabla=\frac{\partial}{\partial r} \mathbf{r}+\frac{1}{r} \frac{\partial}{\partial \theta} \boldsymbol{\theta}+\frac{\partial}{\partial x} \mathbf{x}
$$

Taking the divergent of equation 2.73 to obtain the laplacian differential operator:

$$
\nabla \cdot \nabla=\nabla^{2}=\frac{\partial^{2}}{\partial x^{2}}+\frac{\partial^{2}}{\partial r^{2}}+\frac{1}{r} \frac{\partial}{\partial r}+\frac{1}{r^{2}} \frac{\partial^{2}}{\partial \theta^{2}} .
$$

Applying the laplacian in the wave equation 2.71, we have:

$$
\frac{1}{c_{0}{ }^{2}}\left(\frac{\partial}{\partial t}+U \frac{\partial}{\partial x}\right)^{2} p^{\prime}-\left(\frac{\partial^{2}}{\partial x^{2}}+\frac{\partial^{2}}{\partial r^{2}}+\frac{1}{r} \frac{\partial}{\partial r}+\frac{1}{r^{2}} \frac{\partial^{2}}{\partial \theta^{2}}\right) p^{\prime}=0 .
$$


Equation 2.75 is the wave equation in its more general form that its general solution will be obtained and its behavior assessed. The Mach number on the axial direction of the mean velocity flow field is defined as:

$$
M_{0}=\frac{U}{c_{0}}
$$

Expanding equation 2.75 and rearranging its terms in the following way:

$$
\frac{1}{c_{0}^{2}} \frac{\partial^{2} p^{\prime}}{\partial t^{2}}+\frac{2 M_{0}}{c_{0}} \frac{\partial}{\partial t}\left(\frac{\partial p^{\prime}}{\partial x}\right)+M_{0}^{2} \frac{\partial^{2} p^{\prime}}{\partial x^{2}}=\frac{\partial^{2} p^{\prime}}{\partial x^{2}}+\frac{\partial^{2} p^{\prime}}{\partial r^{2}}+\frac{1}{r} \frac{\partial p^{\prime}}{\partial r}+\frac{1}{r^{2}} \frac{\partial^{2} p^{\prime}}{\partial \theta^{2}} .
$$

To obtain the solution of equation 2.77, the method of separation of variables [62] will be used, where the solution will have the following form:

$$
p^{\prime}(x, r, \theta, t)=X(x) R(r) \Theta(\theta) T(t)
$$

Applying the above solution in equation 2.77, we obtain:

$$
\begin{aligned}
\frac{1}{c_{0}^{2}} X R \Theta T^{\prime \prime}+\frac{2 M_{0}}{c_{0}} X^{\prime} R \Theta T^{\prime}+M_{0}^{2} & X^{\prime \prime} R \Theta T \\
& =X^{\prime \prime} R \Theta T+X R^{\prime \prime} \Theta T+\frac{1}{r} X R^{\prime} \Theta t+\frac{1}{r^{2}} X R \Theta^{\prime \prime} T,
\end{aligned}
$$

where the apostrophe $\left({ }^{\prime}\right)$ indicates the function derivative in respect to its variable. Dividing equation 2.79 by $X R \Theta T$ and rearranging its terms:

$$
\frac{1}{c_{0}^{2}} \frac{T^{\prime \prime}}{T}+\frac{2 M_{0}}{c_{0}} \frac{X^{\prime} T^{\prime}}{X T}+\left(M_{0}^{2}-1\right) \frac{X^{\prime \prime}}{X}=\frac{R^{\prime \prime}}{R}+\frac{1}{r} \frac{R^{\prime}}{R}+\frac{1}{r^{2}} \frac{\Theta^{\prime \prime}}{\Theta} .
$$

The equations of each variable will be separated by its own variable, which implies that the terms of equation with the same variable, will be a separated equation. To obtain the general solutions by separation of variables, the mean flow will be firstly considered equal to zero, then we will assess the mean flow effect on the resulting solution. By making $M_{0}=0$, we have:

$$
\frac{1}{c_{0}^{2}} \frac{T^{\prime \prime}}{T}-\frac{X^{\prime \prime}}{X}=\frac{R^{\prime \prime}}{R}+\frac{1}{r} \frac{R^{\prime}}{R}+\frac{1}{r^{2}} \frac{\Theta^{\prime \prime}}{\Theta},
$$

by doing this way we have four linear differential equations, where the solution of three of them is trivial:

- $X(x)$ :

$$
\frac{X^{\prime \prime}}{X}=-K_{x}^{2}
$$


- $\Theta(\theta)$ :

$$
\frac{\Theta^{\prime \prime}}{\Theta}=-K_{\theta}^{2}
$$

- $T(t)$ :

$$
\frac{T^{\prime \prime}}{T}=-K_{t}^{2}
$$

- $R(r)$ :

$$
\frac{R^{\prime \prime}}{R}+\frac{1}{r} \frac{R^{\prime}}{R}+\frac{1}{r^{2}} \frac{\Theta^{\prime \prime}}{\Theta}=-K_{r}^{2}
$$

where $-K^{2}$ is the separation constant, or wave number of each of the differential equations. The general solution of the three first differential equations is:

$$
F(f)=A e^{i K_{f} f}+B e^{-i K_{f} f}
$$

where $A$ and $B$ are constants evaluated based on the initial and boundary conditions of the problem.

There are other types of solutions, but their nature is not an oscillatory one [62], where the oscillatory type of solution is the one desired due to cyclic and wave like phenomena found in turbofan ducts. The other types of solutions refer to exponential decay or amplification only of the pressure fluctuation or the linear solution. Each term of the solution represents the wave signal traveling in each direction of its independent variable. Naturally the decay and amplification solutions will appear later on with the oscillatory solution that will dictate the modal propagation along the duct.

The mathematical relation for the wave number in the axial direction will be the last to be obtained because it is a function of the wave numbers from the other separable differential equations. Boundary conditions and source characterization allow relations for the wave numbers of the angular coordinate $\theta$ and the time coordinate $t$. The simplest wave number relation to be obtained is the angular coordinate for it is an intrinsic condition of the coordinate, where continuity between the angles $\theta=0$ and $\theta=2 \pi$ is needed, since physically these points are on the same position:

$$
p^{\prime}(x, \theta=0, r, t)=p^{\prime}(x, \theta=2 \pi, r, t) \text { or } \Theta(\theta=0)=\Theta(\theta=2 \pi) .
$$

subsituting equation 2.87 into equation of the general solution 2.86:

$$
\Theta_{1} e^{i K_{\theta} 0}+\Theta_{2} e^{-i K_{\theta} 0}=\Theta_{1} e^{i K_{\theta} 2 \pi}+\Theta_{2} e^{-i K_{\theta} 2 \pi},
$$


taking only the real part of the solution and substituting it in Euler's formula:

$$
A e^{i f}=A \cos (f)+A i \operatorname{sen}(f),
$$

we then obtain the implicit boundary condition of the angular coordinates:

$$
\begin{aligned}
& \Theta_{1} \cos \left(K_{\theta} 0\right)+\Theta_{2} \cos \left(K_{\theta} 0\right)+i\left(\Theta_{1} \operatorname{sen}\left(K_{\theta} 0\right)-\Theta_{2} \operatorname{sen}\left(K_{\theta} 0\right)\right)= \\
& \Theta_{1} \cos \left(K_{\theta} 2 \pi\right)+\Theta_{2} \cos \left(K_{\theta} 2 \pi\right)+i\left(\Theta_{1} \operatorname{sen}\left(K_{\theta} 2 \pi\right)-\Theta_{2} \operatorname{sen}\left(K_{\theta} 2 \pi\right)\right) .
\end{aligned}
$$

Arranging the equations according to the integration constant $\Theta$ and with their real or imaginary condition:

$$
\begin{aligned}
\Theta_{1}\left(\cos \left(K_{\theta} 0\right)-\cos \left(K_{\theta} 2 \pi\right)\right) & =0, \\
\Theta_{2}\left(\cos \left(K_{\theta} 0\right)-\cos \left(K_{\theta} 2 \pi\right)\right) & =0, \\
i \Theta_{1}\left(\operatorname{sen}\left(K_{\theta} 0\right)-\operatorname{sen}\left(K_{\theta} 2 \pi\right)\right) & =0, \\
i \Theta_{2}\left(\operatorname{sen}\left(K_{\theta} 0\right)-\operatorname{sen}\left(K_{\theta} 2 \pi\right)\right) & =0 .
\end{aligned}
$$

The permissible values for the wave number $K_{\theta}$ are integers $m$, positive or not. The physical representation of this restriction of the allowed set of solutions is about the kind of wave that will take part in these solutions of the $\theta$ coordinate. Only waves of integer periods are allowed in these solutions, making the first modal characteristic of the noise propagated in turbofan ducts. We then define these integer values modes $m$ as the circumferential modes, which will have great importance in the noise generation and propagation along the duct.

We then obtain the general solution of equation 2.83 in the circumferential direction:

$$
\Theta(\theta)=\Theta_{1} e^{i m \theta}+\Theta_{2} e^{-i m \theta}
$$

Another solution that is simply obtained is of the temporal coordinate (equation 2.84). Similar to the angular coordinate differential equation, we have the following solution for the temporal coordinate:

$$
T(t)=T_{1} e^{i \omega t}+T_{2} e^{-i \omega t}
$$

where the exponential argument $\omega$ is basically the angular frequency of the time oscillation of the noise generated by the rotor blades.

The solution from equation 2.85 comes from the Frobenius' series, where the Bessel and Neumann equations are derived [62] and are directly related to the circumferential mode $m$ 
which given by the solution of the angular coordinate differential equation. By combining equation 2.85 with equation 2.83 we have:

$$
\frac{R^{\prime \prime}}{R}+\frac{1}{r} \frac{R^{\prime}}{R}+\left(K_{r}^{2}-\frac{m^{2}}{r^{2}}\right)=0
$$

Equation 2.94 is the characteristic equation with eigenvalues $K_{r}$ to be obtained from the boundary conditions on the duct walls at the $r$ coordinate. These eigenvalues may be supressed by the substitution [62] $x=K_{r} r$ and $R(r)=y\left(\frac{x}{K_{r}}\right)$ :

$$
\frac{d^{2} y}{d\left(\frac{x}{K_{r}}\right)^{2}}+\frac{1}{\left(\frac{x}{K_{r}}\right)} \frac{d y}{d\left(\frac{x}{K_{r}}\right)}+\left(K_{r}{ }^{2}-\frac{m^{2}}{\left(\frac{x}{K_{r}}\right)^{2}}\right) y=0,
$$

we then obtain the Bessel differential equation of order $m$ :

$$
\frac{d^{2} y}{d x^{2}}+\frac{1}{x} \frac{d y}{d x}+\left(1-\frac{m^{2}}{x^{2}}\right) y=0
$$

Its general solution is given by:

$$
R(r)=R_{1} J_{m}\left(K_{r} r\right)+R_{2} N_{m}\left(K_{r} r\right),
$$

that is basically the linear combination of the Bessel and Neumann function of order $m$ with their respective integration constants $R_{1}$ and $R_{2}$. The order $m$ of these functions is the same integer that represens the circumferential mode $m$ from the solution of the angular coordinate.

The boundary conditions to be applied now are related to the wall characteristics from the hub and the nacelle when an acoustic wave interacts with its surface. The most usual boundary condition is the hardwall condition, where its mathematical representation is the pressure fluctuation derivative equal zero at the wall locations (Neumann boundary condition):

$$
\left.\frac{d p^{\prime}}{d r}\right|_{r=R_{i}}=\left.\frac{d p^{\prime}}{d r}\right|_{r=R_{o}}=0,
$$

where $R_{i}$ and $R_{o}$ are the hub and nacelle radius respectively. Now defining the following relation between the eigenvalue $K_{r}$ and the relative positions where the boundary conditions are applied:

$$
\sigma_{m n}=K_{r} R_{o}
$$


Also defining the ratio between the internal and external radii of the geometry:

$$
\sigma=\frac{R_{i}}{R_{o}}
$$

where $\sigma=1$ at the nacelle wall.

Substitutingg the general solution 2.97 and equations 2.99 and 2.100 into equation of the boundary condition 2.98, we have:

$$
\left\{\begin{array}{l}
R_{1} J_{m}^{\prime}\left(\sigma_{m n} \sigma\right)+R_{2} N_{m}^{\prime}\left(\sigma_{m n} \sigma\right)=0 \\
R_{1} J_{m}^{\prime}\left(\sigma_{m n}\right)+R_{2} N_{m}^{\prime}\left(\sigma_{m n}\right)=0
\end{array}\right.
$$

By solving the obtained linear system, the following relation is obtained:

$$
\frac{\frac{d}{d r}\left(N_{m}\left(\sigma_{m n} \sigma\right)\right)}{\frac{d}{d r}\left(J_{m}\left(\sigma_{m n} \sigma\right)\right)}-\frac{\frac{d}{d r}\left(N_{m}\left(\sigma_{m n}\right)\right)}{\frac{d}{d r}\left(J_{m}\left(\sigma_{m n}\right)\right)}=0 .
$$

Unfortunately the roots of equation 2.102 cannot be obtained analytically and their eigenvalues must be obtained numerically. For each circumferential mode $m$ there will be more than one root, according to the oscillatory behavior of the Bessel and Neumann equations. As a consequence, we define the radial modes $n$, that are the roots of equation 2.97 numerically obtained. By taking equation 2.97 in the following form that relates $R_{1}$ and $R_{2}$ from equation 2.101:

$$
R(r)=R_{1}\left[J_{m}\left(K_{r} r\right)-\frac{J_{m}^{\prime}\left(K_{r} R_{o}\right)}{N_{m}^{\prime}\left(K_{r} R_{i}\right)} N_{m}\left(K_{r} r\right)\right] .
$$

Up to this point the obtained analytical solution is the following:

$$
\begin{array}{r}
p^{\prime}(x, r, \theta, t)=\left(X_{1} e^{i K_{x} x}+X_{2} e^{-i K_{x} x}\right)\left(\Theta_{1} e^{i m \theta}+\Theta_{2} e^{-i m \theta}\right)\left(T_{1} e^{i \omega t}+T_{2} e^{-i \omega t}\right) \\
\left(R_{1}\left[J_{m}\left(K_{r} r\right)-\frac{J_{m}^{\prime}\left(K_{r} R_{o}\right)}{N_{m}^{\prime}\left(K_{r} R_{i}\right)} N_{m}\left(K_{r} r\right)\right]\right) . \\
p^{\prime}(x, r, \theta, t)=X \Theta T e^{i\left(-\omega t+K_{x} x+m \theta\right)} R_{1}\left[J_{m}\left(K_{r} r\right)-\frac{J_{m}^{\prime}\left(K_{r} R_{o}\right)}{N_{m}^{\prime}\left(K_{r} R_{i}\right)} N_{m}\left(K_{r} r\right)\right]
\end{array}
$$

All the needed relations for the wave number $K_{x}$ were obtained for the case with no axial flow. Substituting on equation 2.81 the obtained solutions for all the coordinates:

- $T(t)$ :

$$
\frac{1}{c_{0}^{2}} \frac{T^{\prime \prime}}{T}=\frac{(-i \omega)^{2}}{c_{0}^{2}}=-\frac{\omega^{2}}{c_{0}^{2}}=-k_{t}^{2} .
$$


- $X(x)$ :

$$
\frac{X^{\prime \prime}}{X}=\left(-i K_{x}\right)^{2}=-K_{x}^{2}
$$

- $R(r)$ :

$$
\frac{R^{\prime \prime}}{R}+\frac{1}{r} \frac{R^{\prime}}{R}+\frac{1}{r^{2}} \frac{\Theta^{\prime \prime}}{\Theta}=-K_{r}^{2} .
$$

By combining the equations and isolating the term $K_{x}$, the relation that defines the wave number in the axial direction is obtained:

$$
K_{x}=\sqrt{\frac{\omega^{2}}{c_{0}^{2}}-K_{r}^{2}}=\sqrt{k_{t}{ }^{2}-k_{r}{ }^{2}} .
$$

For the case where there is uniform flow in the axial direction, and with the same idea of substituting the solutions in the separable variables equations, the following expression for the remaining terms of equation 2.80 is obtained:

$$
\begin{array}{r}
\frac{2 M_{0}}{c_{0}} \frac{X^{\prime} T^{\prime}}{X T}=\frac{2 M_{0}}{c_{0}}\left(-i \omega i K_{x}\right)=\frac{2 M_{0}}{c_{0}} \omega K_{x}, \\
\left(M_{0}^{2}-1\right) \frac{X^{\prime \prime}}{X}=\left(M_{0}{ }^{2}-1\right)\left(i K_{x}\right)^{2}=\left(1-M_{0}{ }^{2}\right) K_{x}{ }^{2} .
\end{array}
$$

Finally, we combine all the terms from the solutions of each coordinate to obtain the wave number $K_{x}$ for the case with axial uniform flow:

$$
K_{r}^{2}+K_{x}^{2}=\left(\frac{\omega}{c_{0}}\right)^{2}-2 M_{0} \frac{\omega}{c_{0}} K_{x}+M_{0}^{2} K_{x}^{2}=\left(\frac{\omega}{c_{0}}-M_{0} K_{x}\right)^{2} .
$$

By isolating the wave number term $K_{x}$ and by applying the sign convention from the solutions exponential arguments $X(x)$ [65]:

$$
\pm K_{x}=\frac{\omega / c_{0}}{\left(1-M_{0}^{2}\right)}\left[-M_{0} \pm \sqrt{1-\left(1-M_{0}^{2}\right)\left(\frac{K_{r} c_{0}}{\omega}\right)^{2}}\right] .
$$

The wave number sign from equation 2.113 refers to the causality condition; the positive sign of $K_{x}$ refers to the wave propagation in the positive axial direction and the negative sign refers to the wave propagation on the negative axial direction. From equation 2.105 we then define the general solution of the wave equation in cylindrical coordinates as the sum of all the particular solutions:

$$
p^{\prime}(x, r, \theta, t)=\sum_{m=-\infty}^{+\infty} \sum_{n=1}^{+\infty} P_{m, n}\left[J_{m}\left(\frac{\sigma_{m n} r}{R_{0}}\right)-\frac{J_{m}^{\prime}\left(\sigma_{m n}\right)}{N_{m}^{\prime}\left(\frac{\sigma_{m n} R_{i}}{R_{0}}\right)} N_{m}\left(\frac{\sigma_{m n} r}{R_{0}}\right)\right] e^{i\left(-\omega t+K_{x} x+m \theta\right)},
$$

where $P_{m, n}$ is the product of all the single coordinates solutions amplitudes, related to the circumferential and radial modes $m$ and $n$ respectively. 


\subsubsection{Subsonic Acoustic Source Characterization}

One of the largest noise sources of an aircraft, the fan noise, is essentially aerodynamically generated noise. Appendix II presents the derivation of the Ffowcs-Williams and Hawkings equation for the mathematical interpretation of the noise sources in volume and surfaces integrals through the polar expansion applied to the free field propagation solution. The resulting noise source terms are due to fluid displacement, pressure distribution and other consequences on the fluid of an immersed solid body moving through it. Such interpretation may be also applied to the noise of the rotor itself and its interaction with the stator $[66,67]$.

\subsubsection{Rotor Blades and Stator Vanes Interaction Generated Noise}

The turbofan rotor and stator interaction noise sources characterized in the appendix II are valid for the flight regimes of a subsonic aircraft. An intense aerodynamic interaction occurs between both parts apart from the rotor and stator themselves generating noise separately.

The aerodynamic noise generated by the rotor blades is mainly of a fundamental frequency and its harmonics, defined by the rotor passing frequency, super-imposed on the broadband content. Among the main noise sources, the most important are the blade thickness associated noise (monopole), blade loading noise (dipole) and the turbulent content associated noise (quadrupole). Operating conditions also contribute to generated noise, like noise due to ingestion of atmospheric turbulence. Different operating conditions, among others may modify the turbofan acoustic signature [66, 67].

The rotor and stator interaction noise is one of the largest noise sources of the turbofan. All the terms of equation II.15 are responsible for noise generated inside the engine duct; the interaction of the flow generated pressure fields produced by the rotor and stator induces loading noise on both. Vorticity wakes produced by the rotor blades impinges on the stator vanes in a cyclic way, modifies the stator surface loading, influencing the monopole and dipole kind noise sources. The main result of the aerodynamic interaction between the rotor and stator is the production of the radial and circumferential (Tyler and Sofrin) modes [69] composing the tonal noise. Directly related to the blade passing fundamental 
frequency and its harmonics, the interaction of the rotor and stator will produce a set of modes, that under certain conditions, will propagate in the upstream and the downstream axial direction.

By modeling the pressure field generated by a rotor alone configuration and making use of the general solution of the wave equation developed in section 2.2.2, the following equation is obtained:

$$
p^{\prime}(x, r, \theta, t)=\sum_{m=-\infty}^{+\infty} \sum_{n=1}^{+\infty} P_{m, n}\left[J_{m}\left(\frac{\sigma_{m n} r}{R_{0}}\right)-\frac{J_{m}^{\prime}\left(\sigma_{m n}\right)}{N_{m}^{\prime}\left(\frac{\sigma_{m n} R_{i}}{R_{0}}\right)} N_{m}\left(\frac{\sigma_{m n} r}{R_{0}}\right)\right] e^{i\left(-\omega t+K_{x} x+m \theta\right)} .
$$

Regarding the generated modes, we now consider only the temporal, radial, and angular parts of the particular solution, where the temporal angular velocity $\omega$ and the circumferential mode $m$ are the values to be obtained due to the rotor and stator interaction and the rotor blade passage fundamental frequency:

$$
p^{\prime}(x, r, \theta, t)=P^{\prime}(r, x) P_{m, n} e^{i(-\omega t+m \theta)},
$$

where $P^{\prime}(r, x)$ is the oscillatory functions of the radial and axial directions. Consider now the passage of several rotor blades through a fixed point, for example, next to a stator vane. Near this point the rotor passage will produce perturbations of the pressure field, where in certain conditions, remains constant if the same point rotates together with the rotor blades. According to the wave equation solution, the value of the pressure fluctuations amplitude is not calculated, because it depends on more complex factors, like the rotor blades and stator vanes geometry description and the flow around them, which occurs in a cyclic way every time a rotor blade passes by a single stator vane. Taking these things into consideration, we may characterize the time term of the exponential argument from the wave equation solution for a fixed point in space:

$$
\omega=h B \Omega
$$

where $h$ is the harmonics of the blade passing frequency, $B$ is the number of rotor blades of the turbofan and $\Omega$ is the angular rotation rate of the rotor shaft, also given by $\Omega=2 \pi f$, where $f$ is the rotation frequency in complete turns per second of the rotor shaft. Consider that the time pressure field solution of the wave equation may be given by the sum of all 
of its harmonics of the rotor turning frequency:

$$
p^{\prime}(x, r, \theta, t)=\sum_{h=1}^{+\infty} P^{\prime}(r, x) P_{m, n, h} e^{i\left(-h B \Omega t+m \theta+\phi_{m, h}\right)},
$$

where $\phi_{m, h}$ is the exponential phase related to the rotor position and their respective harmonic.

In the general wave equation solution, any values for $m$ are considered according with the angular coordinate implicit boundary condition. Consider that at a given time $t$ the rotor position $\theta$ relative to the stator vanes produces a pressure field from their interaction that is equivalent to equation 2.118. Following the same line of thought, the rotor blades and stator vanes are positioned at the same angular distance from its neighbors. Considering that the angular distance between two consecutive stator vanes is given by:

$$
\Delta \theta=\frac{2 \pi}{V}
$$

where $\mathrm{V}$ is the number of stator vanes of the turbofan. The needed amount of time for a pressure state from a particular rotor position to repeat again, when the following rotor blade occupies the same position of its predecessor is given by:

$$
\Delta t=\frac{2 \pi}{V \Omega} .
$$

From the solution of equation 2.118 the pressure field between the reference angular position $\theta$, at the reference time $t$ and after the rotor assumed the angular position $\theta+\Delta \theta$ at the given time $t+\Delta t$, the pressure field will be the same at $t$ and $t+\Delta t$ :

$$
\sum_{h=1}^{+\infty} P^{\prime}(r, x) P_{m, n, h} e^{i\left(-h B \Omega t+m \theta+\phi_{m, h}\right)}=\sum_{h=1}^{+\infty} P^{\prime}(r, x) P_{m, n, h} e^{i\left(-h B \Omega(t+\Delta t)+m(\theta+\Delta \theta)+\phi_{m, h}\right)} .
$$

Rearranging the variation terms $\Delta \theta$ and $\Delta t$ :

$$
\sum_{h=1}^{+\infty} P^{\prime}(r, x) P_{m, n, h} e^{i\left(-h B \Omega t+m \theta+\phi_{m, h}\right)}=\sum_{h=1}^{+\infty} P^{\prime}(r, x) P_{m, n, h} e^{i\left(-h B \Omega t+m \theta+\phi_{m, h}\right)} e^{i(-h B \Omega \Delta t+m \Delta \theta)},
$$

as a consequence, the second exponential term of the right hand side must be equal to one:

$$
e^{i(-h B \Omega \Delta t+m \Delta \theta)}=1
$$

The possible solutions are:

$$
e^{i 2 \pi k}=1
$$


where $k$ is any integer value, positive or negative. Substituting the definitions of $\Delta \theta$ and $\Delta t$ into the equations above:

$$
i(-h B \Omega \Delta t+m \Delta \theta)=i\left(-h B \Omega \frac{2 \pi}{V \Omega}+m \frac{2 \pi}{V}\right)=i 2 \pi k
$$

and finally isolating the $m$ term:

$$
m=h B+k V \text { where } k=-\infty, \ldots,-1,0,1, \ldots,+\infty \mid k \in \mathbb{Z}
$$

The values obtained for $m$ from equation 2.126 are the possible values for the circumferential mode $m$ related to the $\Delta \theta$ and $\Delta t$ cyclic conditions. Such circumferential modes are commonly known as the Tyler and Sofrin interaction modes. These are all the modes obtained by the interaction between the rotor blades and the stator vanes associated with their fundamental frequency and its harmonics. There are special cases for the equation 2.126, for example, when there are no stator vanes, where the generated modes are solely multiples of the number of rotor blades and a single mode per harmonic $(m=B h)$. Another example is the case where there are no rotor blades and only stator vanes, where mathematically circumferential modes may exist according to equation 2.126, but physically inconsistent because no frequency is associated with the stator vanes only configuration.

Substituting equation 2.126 into the wave equation general solution 2.2.2, it is obtained:

$$
\begin{aligned}
& p^{\prime}(x, r, \theta, t)= \\
& \quad \sum_{h=1}^{+\infty} \sum_{n=1}^{+\infty} \sum_{k=-\infty}^{+\infty} P_{m, n}\left[J_{m}\left(\frac{\sigma_{m n} r}{R_{0}}\right)-\frac{J_{m}^{\prime}\left(\sigma_{m n}\right)}{N_{m}^{\prime}\left(\frac{\sigma_{m n} R_{i}}{R_{0}}\right)} N_{m}\left(\frac{\sigma_{m n} r}{R_{0}}\right)\right] e^{i\left(-h B \Omega t+K_{x} x+\theta(h B+k V)\right)} .
\end{aligned}
$$

Equation 2.127 is the general solution for the case where the tonal noise is generated by the rotor and stator interaction at the axial coordinate origin, where the acoustic waves propagate in the positive and negative axial directions. The Tyler and Sofrin mode generation rule makes the selection of the generated circumferential modes. Unfortunately there is not a mathematical relation that selects which radial modes will be generated by the rotor and stator interaction, or even for the case where there are only the rotor blades. The amplitude of the circumferential modes, along with its radial mode counterpart depends on the aerodynamic properties of the rotor and stator and must be obtained by numerical 
or experimental means for their propagation analysis [68]. The physical solution will be considered as the real part of the above equation.

\subsubsection{Tonal Modal Noise Propagation Through the Nacelle-Hub Duct}

Based on the solution of the wave equation with convective effects in cylindrical coordinates, and considering the set of solutions that follows the Tyler and Sofrin circumferential modes selection rule, it is possible to characterize the generated tonal noise and its propagating behavior through the nacelle-hub duct. With the duct propagation theory, the set of modes that effectively composes the noise becomes more restricted, where only generated and propagated modes will irradiate outside the duct.

\subsubsection{Propagating Modes Definition}

By modeling the turbofan nacelle and hub as a circular uniform duct with constant axial mean flow and with the hardwall boundary condition, equation 2.113 is the relation that will dictate mode behavior at these flow conditions. The generated modes are associated with circumferential and radial characteristics that will compose each harmonic of the tonal noise.

According to equation 2.127, the modes of a given particular solution will propagate in an oscillatory way without changes of its amplitude, if all the spatial and temporal variables and arguments of the exponential terms are real. This assertion will hold true for the wave number terms from the circumferential, temporal and radial wave numbers, considering that the Bessel and Neumann eigenvalues are real values. The wave number associated with the axial direction and argument of the exponential may be of complex type.

Taking the wave number equation of the axial direction 2.113, repeated here for the sake of clarity and remembering that the sign change implies the wave propagation direction in the axial direction:

$$
\pm K_{x}=\frac{\omega / c_{0}}{\left(1-M_{0}^{2}\right)}\left[-M_{0} \pm \sqrt{1-\left(1-M_{0}^{2}\right)\left(\frac{K_{r} c_{0}}{\omega}\right)^{2}}\right] .
$$


Both real and imaginary values may be obtained from the square root of equation 2.128. Real values for the wave number induces in the solution oscillatory behavior only, but when these arguments have complex values, the solution of the wave equation will have a decaying or amplifying effect on the wave propagation.

Taking into account the wave equation solution and remebering the signal convention for both the wave equation solution and the axial wave number equation, only complex arguments related to wave decay are allowed, keeping the physical coherence in the thermodynamic point of view. Now isolating the square root argument from equation 2.128, we have the following conditions for mode propagation:

$$
\left(1-M_{0}^{2}\right)\left(\frac{K_{r} c_{0}}{h B \Omega}\right)^{2}=\left\{\begin{array}{l}
<1 \text { cut on, } \\
=1 \text { critical value } \\
>1 \text { cut off. }
\end{array}\right.
$$

We now define that when the value of the above expression is lower than one, the square root of equation 2.113 will be of real type and there will be no dampening of the propagating wave on equation 2.127, we will define the propagating mode to be cut-on. When the expression above has values higher than one, the square root will be of imaginary type and the exponential decay term will appear in equation 2.127; the generated modes will cut-off. There are the critical modes, that are also of cut-on type, when equation 2.129 is equal to unity.

According to equation 2.129, the most influential factors on mode propagating behavior are basically thermodynamic (sound speed $c_{0}$ ), operational conditions of the aircraft (axial Mach number) and values that are related to the engine design (number of rotor blades and stator vanes, rotor shaft angular speed and hub and nacelle radii). The turbofan configuration is directly related to the radial wave number, that must be obtained numerically for given radial $n$ and circumferential $m$ modes that were given by equation 2.126), and then compared with the critical value. Substituting equation 2.99 into equation 2.129 on the critical condition and isolating the critical eigenvalue term $\sigma_{m n, c}$ :

$$
\sigma_{m n, c}=\frac{R_{o} \Omega h}{c_{0}\left(1-M_{0}^{2}\right)^{1 / 2}} .
$$

A kinematic analysis may help the understanding of the cut-on and cut-off aerodynami- 
cally generated modes due to rotor and stator interaction. Taking the exponential argument of the general solution of the wave equation 2.127, with the generated modes from the rotor and stator interaction and considering the argument as a constant, we have the isovalue of the resulting wavefront of the circumferential, axial and temporal solutions [66]:

$$
-h B \Omega t+K_{x} x+\theta(h B+k V)=\phi .
$$

By isolating the $\theta$ term and differentiating it in respect to time to obtain the circumferential phase velocity at a fixed plane perpendicular to the $x$ axis we have:

$$
(h B+k V) \frac{d \theta}{d t}=h B \Omega .
$$

Now multiplying both sides of the above expression by the external radius (nacelle inner wall), we define the phase velocity:

$$
R_{o} \frac{d \theta}{d t}=U_{m}=\frac{h B U_{R_{o}}}{h B+k V}
$$

Rearranging:

$$
\frac{U_{m}}{U_{R_{o}}}=\frac{h B}{h B+k V}
$$

Equation 2.134 is the ratio of the circumferential speeds of a given circumferential mode $m$ and the rotor blades circumferential speed. Such expression implies that for a given harmonic, the condition to a given mode spin rate in the same rate, the ratio of the number of lobes of this rotor only generated mode and the one from the interaction must be the same phase speeds. As a consequence, the interaction generated modes that have lower values than the rotor only generated modes have higher circumferential phase speeds that can be higher than the sound velocity itself.

Combining equations 2.134 and 2.129 at the critical condition, yields:

$$
U_{m, c}=\left(1-M_{0}^{2}\right)^{1 / 2} \frac{\sigma_{m n}}{m} c_{0}
$$

equation 2.135 expresses the needed circumferential phase speed the mode $m$ must have to be of cut-on type. Equation 2.135 can also be simplified for no flow conditions $M_{0}=0$ and now taking the ratio $\sigma_{m n} / m$ as near one for large $m$ values, the following expression is obtained:

$$
U_{m, c}=c_{0} .
$$


As a rule-of-thumb, equation 2.136 says that for a given circumferential mode, it will propagate only if it has at least sonic phase speed. This also implies that the propagation condition for a rotor only generated mode is that the rotor must spin at least sonic tip speed. But in the case between the rotor and the stator, according to equation 2.134, the rotor blades do not necessarily have sonic phase speed to generate propagating modes, because some interaction generated modes may have supersonic phase speed to keep their respective harmonic frequency.

\subsubsection{Modal Propagation Angles}

Similar to the linearization process was done on the governing equations to obtain analytical results concerning the modal propagation in ducts, its geometric characteristics may be obtained through simple geometric analysis of the results from the wave equation solution.

Again regarding constant cross section ducts, we consider that the wave fronts that compose the circumferential $m$ modes behave like plane waves, that propagate with a given angle $\phi_{i}$ in respect to a given $i$ coordinate. With simple geometrical manipulations, we may obtain this wave front angle with the $i$ coordinate [70]:

$$
\cos \left(\phi_{i}\right)=\frac{K_{i}}{\sqrt{\sum_{i} K_{i}^{2}}} .
$$

In the cylindrical duct case from the previous sections, we have the following wave numbers: $K_{i}=K_{r}, K_{x}$. The wave front angle with the axial coordinated is given by:

$$
\cos \left(\phi_{x}\right)=\frac{K_{x}}{\sqrt{K_{r}^{2}+K_{x}^{2}}} .
$$

Substituting the denominator of equation 2.138 with the wave numbers from the coordinates given by equation 2.112 and by the definition of the temporal wave number, i.e., $K_{t}=\omega / c_{0}$ yields:

$$
\cos \left(\phi_{x}\right)=\frac{K_{x}}{K_{t}-M_{0} K_{x}}
$$

Now substituting the respective wave numbers:

$$
\cos \left(\phi_{ \pm x}\right)=\frac{-M_{0} \pm \sqrt{1-\left(1-M_{0}^{2}\right)\left(K_{r} / K_{t}\right)^{2}}}{1 \mp M_{0} \sqrt{1-\left(1-M_{0}^{2}\right)\left(K_{r} / K_{t}\right)^{2}}}
$$


The following form of equation 2.140 as the source defined for the wave number $K_{t}$ and with the eigenvalues calculated for $\sigma_{m n}$ may be obtained:

$$
\cos \left(\phi_{ \pm x}\right)=\frac{-M_{0} \pm \sqrt{1-\left(1-M_{0}^{2}\right)\left(\frac{\sigma_{m n} c_{0}}{R_{o} B \Omega h}\right)^{2}}}{1 \mp M_{0} \sqrt{1-\left(1-M_{0}^{2}\right)\left(\frac{\sigma_{m n} c_{0}}{R_{o} B \Omega h}\right)^{2}}} .
$$

\subsubsection{Two Dimensional Cascade Approximation}

More simplifications will be used on the modal analysis to provide a better validation of the numerical results of this work. We will consider the two dimensional simplification of the numerical cases, where the radial element of the flow can be removed, where the lack of source modeling and information may interfere with the validation process, without losing much of the physical insight.

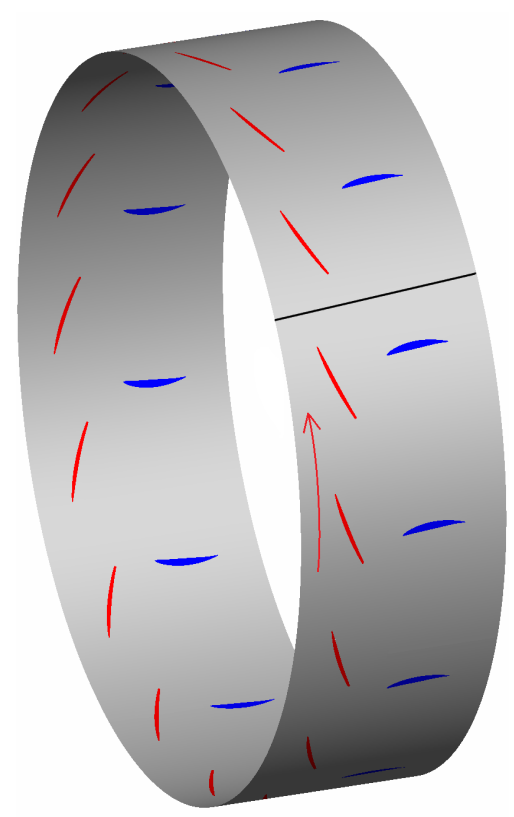

Figure 2.2: Thin annular duct approximation.

The proposed approximation is carried out by taking the limit $R_{i} \rightarrow R_{o}$, i.e., making the hub radius almost as large as the inner nacelle wall radius, thus eliminating any higher modal radial content from the propagation mechanics, resulting in the thin annular duct condition $[71,72]$, where the flow is basically two dimensional. Such approximation corresponds directly with the Cartesian two dimensional case with cyclic boundary conditions at the 
upper and lower positions of the domain.

The mathematical consequences on the wave equation solution 2.127 result in a simplification of the eigenvalues [72] of the linear system of equations 2.101:

$$
\left\{\begin{array}{l}
\sigma_{m n}=m \text { for } n=1, \\
\sigma_{m n} \gg \sigma_{m n, c} \text { for } n>1 .
\end{array}\right.
$$

The radial modes $n$ associated with the circumferential modes $m$ that are larger than one will necessarily be of cut-off type. This means that the thin annular duct approximation is equal to a two dimension case of cascades. The calculated eigenvalues are shown in tables I.1 and I.2 in the appendix I.

\subsubsection{Circumferential Modes Measurement by Doppler Effect}

Regarding ducts and thin annular ducts, the circumferential mode measurement of all its harmonics structure is possible by the use of the Doppler effect on the pressure signal measurement of the modal propagation field.

According to equation 2.134, each mode associated with a given harmonic will have its phase speed given by the ratio the number of rotor blades with the circumferential mode value and with its respective harmonic number. From the exponential argument of equation 2.127, we have:

$$
e^{i\left(-h B \Omega t+K_{x} x+\theta m\right)}=e^{i\left(-h B \Omega t+\theta m+\phi_{x}\right)} .
$$

This part of the wave equation solution represents the time oscillation at a given position in space. The time varying pressure signal will be given at same circumferential positions of the angular coordinate $\theta$ at the plane perpendicular to a fixed axial position, where the difference from two points will be due to phase angle $\phi_{x}$. Making the angular position of the pressure signal measurement to change with time at a constant angular velocity $\frac{d \theta(t)}{d t}=\dot{\theta}$, and by defining this velocity as a fraction of the rotor blades angular frequency $\varphi=\frac{\Omega}{\dot{\theta}}$, we have:

$$
e^{i\left(-h B \Omega t+\theta(t)+\phi_{x}\right)}=e^{i\left(-h B \Omega t+\dot{\theta} m t+\phi_{x}\right)}=e^{i\left(\left(-h B \Omega+\frac{\Omega m}{\varphi}\right) t+\phi_{x}\right)} .
$$

By applying the Fourier transform of equation 2.144:

$$
\mathcal{F}\left(e^{i\left(\left(-h B \Omega+\frac{\Omega m}{\varphi}\right) t+\phi_{x}\right)}\right)=\sqrt{2 \pi} \delta\left(-h B \Omega+\frac{\Omega m}{\varphi}\right) .
$$


The result obtained shows that the exponential time argument will result in a Dirac delta function at the modulus of the frequency itself. By doing this, there will be a shift in

the frequency of the measured pressure signal $\frac{\Omega m}{\varphi}$ around its corresponding harmonic [73, 74]. As a consequence, the frequency shift of a pressure measurement probe that spins at the same angular direction as the rotor will have a decrease in its frequency for a given circumferential mode $m$ with positive value, while a mode $m$ with negative value will have an increase in its frequency. Certain care must be taken into account for determining the value of $\dot{\theta}$ in the case where two consecutive harmonics have decomposed modes that may intersect each other, mixing the measurement. The value of $\dot{\theta}$ also must be fast enough to make possible the distinction between the modes of a given harmonic.

\subsection{Noise Generation by Supersonic Sources and Prop- agation in Ducts}

With the introduction of the turbofan engines in the 1970s and the increase of the fan bypass ratio, the fan tip speeds also increased, reaching supersonic levels during some flight phases (take-off and climb). This produces a system of shock-waves and expansion waves fixed to the rotor blades that propagates in the upstream direction that finally radiates to the environment as the prevalent source of noise in these conditions, affecting both the community and cabin noise levels.

In nominal operating and perfect manufacturing conditions the noise produced by supersonic blades has a single tone noise related to the blade passing fundamental frequency and its harmonics that is associated with a circumferential pressure profile that resembles a sawtooth pattern, shown in figure 2.3. But in realistic conditions, due to manufacturing processes and extended usage, minimal blade to blade differences are produced in the stagger angle, camber, thickness and blade spacing, slightly affecting the system of shock and expansion waves in the rotor blades region, as shown in figure 2.4. The mechanisms of this particular noise generation and the shock-waves behavior in ducts have been addressed theoretically and experimentally since the 1970 s $[75,72,77,78,79]$, and are phenomenologically well understood. 
a)

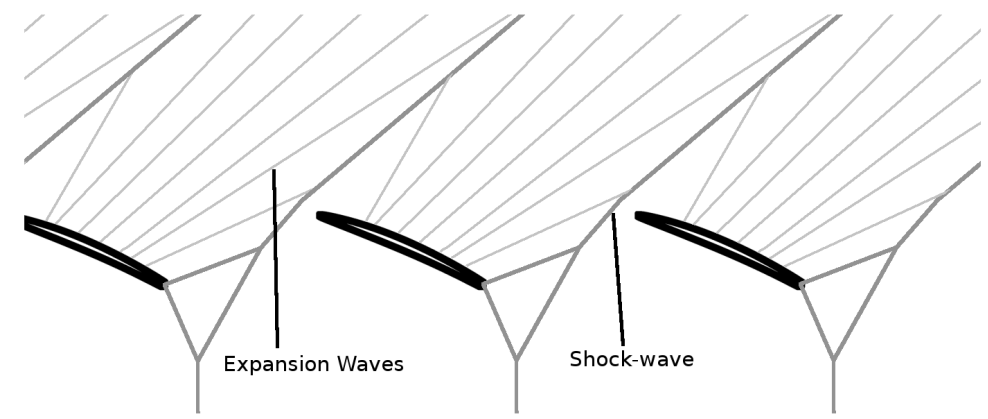

b)

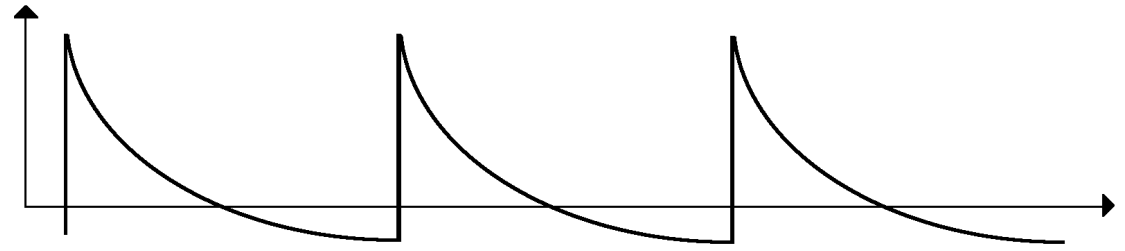

Figure 2.3: Uniform system of shock and expansion waves generated by the supersonic tangential velocity of the rotor tips. (a) Shock and expansion waves pattern around the rotor blades. (b) Circumferential pressure profile near the rotor blades leading edges.

a)

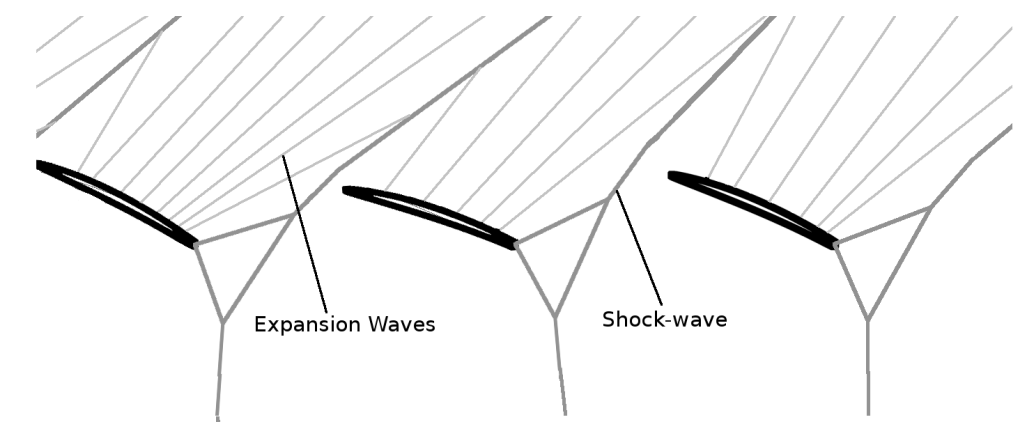

b)

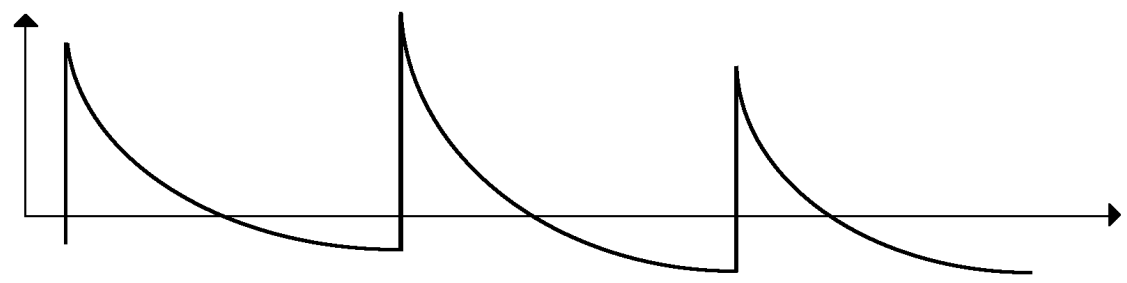

Figure 2.4: Shock-waves and expansion waves distortions due to rotor blade to blade nonuniformity. (a) Shock and expansion waves pattern around the rotor blades. (b) Circumferential pressure profile near the rotor blades leading edges.

Due to the non-uniform pressure pattern at each blade stage, the fundamental tonal frequency changes to the rotor shaft rotation frequency, where the resultant pressure pattern repeats only after a full shaft turn. The perceived noise resultant from this interaction is commonly referred to as the multiple tone noise or buzz-saw noise, for its resemblance to 
the sound produced by a circular buzz-saw. These effects vary from engines of the same model due to these slight rotor to rotor blades differences, making it difficult to obtain an ultimate method to predict this kind of noise for a given turbofan engine.

\subsubsection{Regular Shock-waves Propagation in Ducts}

Several methodologies have been applied to predict the sound pattern at the end of an engine duct. For regular sawtooth wave patterns propagating through ducts, nonlinear analytical methods were proposed [75, 79, 72].

Initially consider the analytic relations that describe the decay rate of a sawtooth shockwave, as shown in figure 2.5. An analytical relation [72] for a sawtooth wave traveling in the medium, where it is considered a weak shock in a perfect gas and that describes at a fixed time the decay of a sawtooth wave pattern along a duct, is given by:

$$
\frac{d(1 / z)}{d t}=\frac{(\gamma+1)}{2 \gamma} \frac{c_{0}}{\lambda}
$$

where $\mathrm{z}$ is the non-dimensional pressure jump of the sawtooth wave $\Delta p / p_{0}, \gamma$ is the gas specific heat ratio and $\lambda$ is the wave length of the sawtooth wave generated by the supersonic rotor blades. Integrating equation 2.146 with respect to time and considering for the integration interval the shock-wave amplitude $z_{i}$ for an initial time $t_{0}$, where we will consider $t_{0}=0$, up to an arbitrary time $t$ yields:

$$
\frac{\Delta p(t)}{p_{0}}=z(t)=\left[\frac{1}{z_{i}}+\frac{c_{0} t}{\lambda} \frac{(\gamma+1)}{2 \gamma}\right]^{-1} .
$$

The needed values for equation 2.147 are the initial shock-wave amplitude $z_{i}$ and its wave length $\lambda$ located at the plane perpendicular to the flow direction, upstream to the rotor blades, and the shock-wave propagation time $t$.

We may use equation 2.147 for a regular shock-wave system propagating in a duct, i.e., the turbofan nacelle and hub duct. At these conditions, the wave fronts will propagate through an helicoidal path along the duct and its propagation will have a retarded effect due to the incoming flow.

From figure 2.6, we obtain the following relation between the flow angles:

$$
\phi+\beta+\mu=\pi
$$




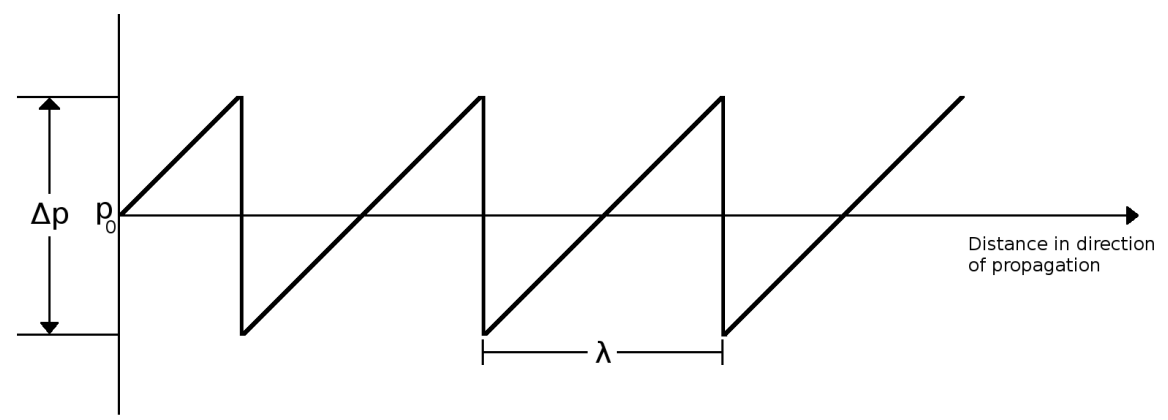

Figure 2.5: Repeated sawtooth wave pattern generated by supersonic rotor blade cascades.

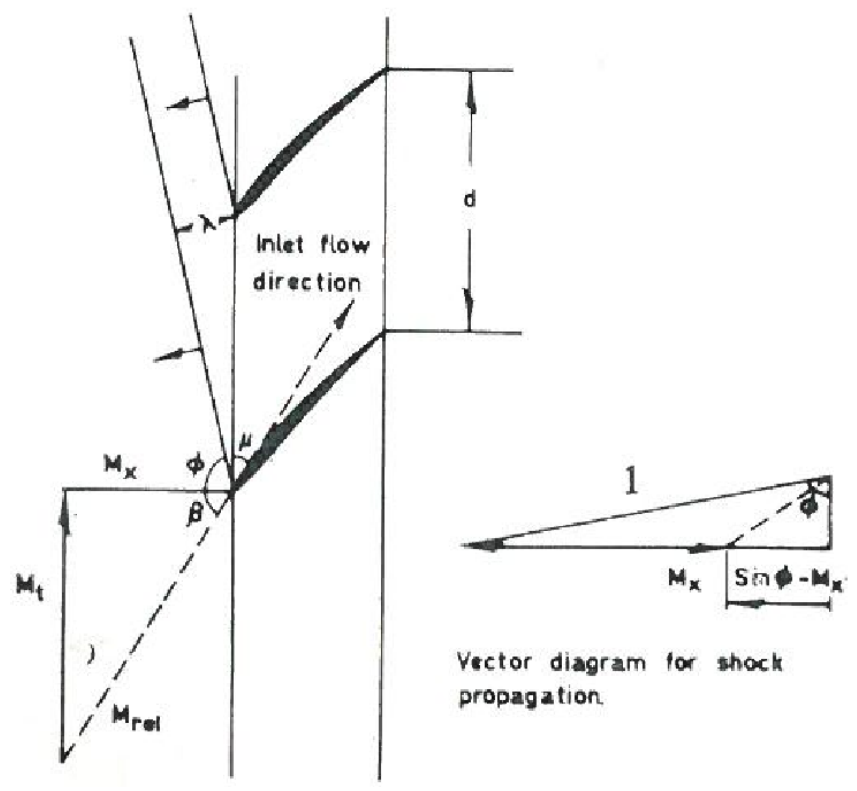

Figure 2.6: Geometrical details of the system of regular sawtooth shock-waves at the upstream rotor region [76].

where $\phi$ is the angle between the shock-wave and the duct axis, $\beta$ is the angle between the flow relative to the rotor blade with the duct axis and $\mu$ is the Mach angle of the shock-wave. By applying the sine on equation 2.148 and with the mathematical identity of the sum of the angles of the sine argument, we have:

$$
\sin (\phi)=\sin (\mu) \cos (\beta)+\cos (\mu) \sin (\beta) .
$$

Also applying the same idea as above but with the cosine on equation 2.148, we have:

$$
\cos (\phi)=\sin (\mu) \sin (\beta)-\cos (\mu) \cos (\beta)
$$

The two obtained expressions above will be useful to define a relation for the propagation time of the shock-wave and the traveled axial distance $x(t)$. 
From figure 2.6 we have the following relations:

$$
\begin{aligned}
& \cos (\beta)=\frac{M_{x}}{M_{r e l}}, \\
& \sin (\mu)=\frac{1}{M_{r e l}},
\end{aligned}
$$

where $M_{x}$ is the axial Mach number of the flow and $M_{r e l}$ is the Mach number of the flow relative to the rotor blades, given by:

$$
M_{r e l}^{2}=M_{x}^{2}+M_{t}^{2}
$$

where $M_{t}$ is the Mach number of the tangential speed of the rotor blades. By combining equations 2.151 and 2.152 into equations 2.149 and 2.150 , we have respectively:

$$
\begin{aligned}
& \sin (\phi)=\frac{1}{M_{r e l}^{2}}\left[M_{x}+M_{t}\left(M_{r e l}^{2}-1\right)^{1 / 2}\right], \\
& \cos (\phi)=\frac{1}{M_{r e l}^{2}}\left[M_{t}-M_{x}\left(M_{r e l}^{2}-1\right)^{1 / 2}\right] .
\end{aligned}
$$

According to figure 2.6, we may define the Mach number of the shock-wave relative to the duct:

$$
M_{u}=\sin (\phi)-M_{x}
$$

Again, according to figure 2.6 we may associate the wave length $\lambda$ with the tangential distance $d$ of two equivalent and neighboring blade points:

$$
\lambda=d \cos (\phi)
$$

Also defining the relation between the axial distance $x(t)$ traveled by the shock-wave and the propagation time of that distance:

$$
x(t)=M_{u} c_{0} t
$$

by rearranging and multiplying both sides by the wave length $\lambda$, we have:

$$
\frac{\lambda}{c_{0} t}=\frac{M_{u} \lambda}{x}
$$

Substituting equations 2.154 and 2.155 into equation 2.157:

$$
\frac{\lambda}{c_{0} t}=\frac{d}{x} \cos (\phi)\left(\sin (\phi)-M_{x}\right)
$$


Now defining the following term $F\left(M_{r e l}, M_{x}\right)$ :

$$
F\left(M_{r e l}, M_{x}\right)=\cos (\phi)\left(\sin (\phi)-M_{x}\right)
$$

By substituting equation 2.153 into equation 2.159 and rearranging its terms, we obtain:

$$
F\left(M_{r e l}, M_{x}\right)=\frac{\left(M_{r e l}^{2}-1\right)^{1 / 2}}{M_{r e l}^{4}}\left[M_{x}\left(M_{r e l}^{2}-1\right)^{1 / 2}-M_{t}\right]^{2},
$$

where:

$$
\frac{\lambda}{c_{0} t}=\frac{d}{x} F\left(M_{r e l}, M_{x}\right)
$$

Substituting equation 2.161 into equation 2.147:

$$
\frac{\Delta p(t)}{p_{0}}=\left[\frac{1}{z_{i}}+\frac{x}{F\left(M_{r e l}, M_{x}\right) d} \frac{(\gamma+1)}{2 \gamma}\right]^{-1} .
$$

Equation 2.162 states the decay of a rotor blade generated shock-wave that propagates at an axial distance along the duct [76], a function of the flow variables $F\left(M_{r e l}, M_{x}\right)$. Note that equation 2.162 is relevant to shock-wave propagation through the duct only, the generation process and shock-wave amplitudes must be obtained from other means, such as from CFD or experiment.

\subsubsection{Non-Regular Shock-waves Propagation in Ducts}

In the case studied in section 2.3.1 the realistic buzzsaw noise signature was not considered. When the rotor blades show slight geometrical differences between them, some slight differences between the shock-waves are also produced, resulting in differences at their propagation speeds and propagation angles. In this case, some phenomena may occur when a neighboring shock-wave travels at a higher velocity than its counterparts. If the duct is long enough and the mean values of the shock-waves pressure jump [58], shock coalescence between faster and slower shock-waves may occur. Two neighboring shock-waves become one shock-wave with a distinct propagation speed and decay rate.

Modifications can be made to the theory of propagation and decay of shock-waves in a duct, as defined in section 2.3.1, where it is possible to predict the propagation of a system of non-regular sawtooth shock-waves, which corresponds to the noise signal produced by supersonic rotor blades with geometrical differences. Algorithms for prediction of these 
system of non-regular shock-waves are available in literature [57, 58, 59]. Some of these algorithms are applied on the time domain $[75,58]$ or on the frequency domain $[59,60]$.

Consider a frame of reference traveling at the sound speed given by the mean temperature of the inlet region and at an helicoidal path through the duct. The propagation angle $\phi$, defined by equations 2.153 and that is used under the following form:

$$
\phi=\operatorname{acos}\left[\frac{1}{M_{r e l}^{2}}\left(M_{t}-M_{x}\left(M_{r e l}^{2}-1\right)^{1 / 2}\right)\right],
$$

where $\phi$ is the angle of propagation between a given shock-wave and the duct axial direction, given by the flow inlet conditions and the rotor blades tangential speed.

Now consider that the expansion waves between the shock-waves result in equal gradients among them as time passes, the modulus of the decay rate of the maximum value of the shock-waves and the growth rate of its minimum value are equal [58]. As a consequence, the mean pressure of the shock-waves pressure jump remains constant while the propagation occurs. When a sawtooth shock-wave has a mean pressure that differs from the flow mean pressure, it propagates at a constant but different speed from the system of coordinates:

$$
v\left(p_{m}\right)=\frac{(\gamma+1) c_{0}}{2 \gamma p_{0}} \Delta p_{m}
$$

where the subscripts 0 reffer to the duct inlet values and $\Delta p_{m}$ is the mean pressure difference of the shock-wave $p_{m}$ and the inlet duct pressure $p_{0}$. A useful form for equation 2.164 is the difference of the two neighboring shock-waves propagation speeds:

$$
\Delta v\left(p_{m 1}, p_{m 2}\right)=\frac{(\gamma-1) c_{0}}{2 \gamma p_{0}}\left(p_{m 1}-p_{m 2}\right)
$$

where the mean pressure of each shock-wave is $p_{m 1}$ and $p_{m 2}$.

If one of two neighboring shock-waves have different mean pressure and the pursuer shock has higher mean pressure than the followed shock, inevitably at a given coalescence time $t_{c}$ they will merge into a new shock-wave, that will propagate on the same conditions of the other shocks before the coalescence. The coalescence time is given by:

$$
t_{c}=\frac{\lambda}{\Delta v\left(p_{m 1} p_{m 2}\right)} .
$$

Another expression needed to describe the propagation and coalescence phenomena of sawtooth shock-waves, is the axial distance equivalent to its propagation time. Already defined 
by equation 2.161, repeated here for the sake of clarity:

$$
\frac{\lambda}{c_{0} t}=\frac{d}{x} F\left(M_{r e l}, M_{x}\right)
$$

The pressure jump from the resulting shock-wave of two coalescing ones is simply the sum of both at the coalescing time and the resulting mean pressure is the coalescing shocks weighted average where the pressure jumps are their respective weights.

To become useful so that comparisons can be made with the numerical results, the circumferential components [75] of the velocities and all the other variables are used:

$$
\begin{array}{r}
v_{y}\left(p_{m}\right)=\frac{v\left(p_{m}\right)}{\cos (\phi)}, \\
\Delta v_{y}\left(p_{m 1}, p_{m 2}\right)=\frac{\Delta v\left(p_{m 1}, p_{m 2}\right)}{\cos (\phi)}, \\
t_{c}=\frac{\lambda}{\Delta v\left(p_{m 1}, p_{m 2}\right)}=\frac{d}{\Delta v_{y}\left(p_{m 1}, p_{m 2}\right)} .
\end{array}
$$

The decay of each sawtooth shock-wave is the same one used in the case where the shock distribution is totally homogeneous. Note that the case where there are only identical shock-waves both in mean pressure and shock amplitude reduces to the use of equation 2.147 only.

The input data of the analytical method is the shock-wave circumferential distribution before the rotor, at a given position where the shock-waves are already weak. The circumferential distance distribution between the shocks is approximately the same, where they can be regarded as the rotor-to-rotor circumferential distance. The main difference between them is the mean pressure and shock amplitude.

With all the needed relations derived, it is possible to make a simple algorithm that will describe the pressure field of the propagating shock-waves in the inlet direction. The main idea of this algorithm is that at a given initial circumferential shock-wave condition the prediction of the lowest shock coalescence must be made to be able to assemble a new circumferential shock-wave distribution. This distribution is now considered as the new initial condition and their decay and coalescence time is again calculated until the desired axial distance is reached. This method was implemented in a purely serial code in Matlab R2013b for Linux 64 bits and the execution time to achieve the circumferential shock 
distribution for the farthest axial distance to be compared with the numerical simulation took less than a second. 


\section{Chapter 3}

\section{Non-linear Gas Dynamics with Expansional Viscosity}

This chapter describes the expansional viscosity model [20], regarding a non-Stokesian fluid, where the state of thermodynamic equilibrium is not instantaneously achieved. Dissipative effects related to the dilatation and contraction of the fluid have strong influence on the equilibrium of forces and energy conservation on the medium. The model is described and its nondimensional parameters are identified. A one dimensional linear stability analysis is carried out in the frequency domain as a preliminary study for the model behavior in numerical simulations.

\subsection{The Stress Tensor}

Consider the constitutive equation for a stress tensor that describes a viscous Newtonian fluid in the most general form [18]:

$$
\sigma_{i j}=-p_{0} \delta_{i j}+\tau_{i j}
$$

where $p_{0}$ in this context is the equilibrium pressure or the thermodynamic pressure and $\tau_{i j}$ is the nonequilibrium part or the flow part of the stress tensor given by the generalized Newton viscosity law [18]:

$$
\tau_{i j}=\eta_{i j k l} D_{k l},
$$


where $D_{k l}$ is the rate of strain tensor:

$$
D_{i j}=\frac{1}{2}\left(\frac{\partial u_{j}}{\partial x_{i}}+\frac{\partial u_{i}}{\partial x_{j}}\right) .
$$

Here we will consider $\eta_{i j k l}$ as an isotropic fourth order viscosity tensor where its most general form is given by [61]:

$$
\eta_{i j k l}=\lambda \delta_{i j} \delta_{k l}+\beta \delta_{i k} \delta_{j l}+\gamma \delta_{i l} \delta_{j k}
$$

where $\lambda, \beta$ and $\gamma$ are material constants of a homogeneous Newtonian fluid.

By substituting equation 3.4 into equation 3.2, and taking into account the symmetry of the rate of strain tensor $D_{i j}$, we have:

$$
\tau_{i j}=\lambda \delta_{i j} D_{k k}+(\beta+\gamma) D_{i j}
$$

Defining $\beta+\gamma=2 \mu$ at the shearing strain term:

$$
\tau_{i j}=\lambda \delta_{i j} D_{k k}+2 \mu D_{i j}
$$

remembering that the term $D_{k k}$ is the velocity divergent $\nabla \cdot \mathbf{u}$ and that it physically represents the rate of dilatation or contraction of the fluid element per unit of volume.

Now we define $\mu$ as the shear viscosity of the Newtonian fluid and $\lambda$ as its bulk or expansional modulus. Substituting equation 3.6 into equation 3.1, we have:

$$
\sigma_{i j}=-p_{0} \delta_{i j}+\lambda \delta_{i j} \nabla \cdot \mathbf{u}+2 \mu D_{i j}
$$

\subsubsection{Mechanical Pressure}

We may define the flow mechanical pressure from the trace of the tensor $\sigma_{i j}[18]$ :

$$
p=-\frac{\operatorname{tr}(\sigma)}{3} \text {. }
$$

By combining equation 3.8 with equation 3.7 , we have:

$$
p=p_{0}-\lambda \nabla \cdot \mathbf{u}-\frac{2}{3} \mu \nabla \cdot \mathbf{u},
$$

rearranging the obtained equation:

$$
p=p_{0}-\left(\lambda+\frac{2}{3} \mu\right) \nabla \cdot \mathbf{u} .
$$


We now define the term $\kappa=\lambda+\frac{2}{3} \mu$ as the second viscosity coefficient or expansional viscosity that in the constitutive equation for the mechanical pressure is given by:

$$
p=p_{0}-\kappa \nabla \cdot \mathbf{u}
$$

Equation 3.11 shows that the difference between the mechanical pressure, or flow pressure, and the thermodynamic pressure, or equilibrium pressure, is directly related to the second viscosity coefficient $\kappa$. The stated equilibrium deviation due to the presence of the flow is given by $\nabla \cdot \mathbf{u}$, that occurs in compressible flows with high frequencies or dilatation and contraction amplitudes, like for exemple, the acoustic propagation of waves with high frequencies, high frequency oscillation of bubles in a liquid fluid or yet wave propagation at large distancies.

\subsubsection{The Stokesian Fluid}

In Newtonian and Stokesian fluids it is considered that the deviation from the thermodynamic equilibrium induced on a fluid by the flow or some excitation is too small, when compared with the dilatation or contraction rate of the same characteristic time of the flow. This is a typical laboratory condition and is usually found in most of the low compressible flow cases, where:

$$
\kappa \approx 0
$$

resulting in:

$$
\lambda=-\frac{2}{3} \mu
$$

in equation 3.11. This condition is the Stokes' hypothesis, where the mechanical pressure is equal the thermodynamic pressure:

$$
p=p_{0}
$$

and equation 3.7 results in the following form:

$$
\sigma_{i j}=-p_{0} \delta_{i j}-\frac{2}{3} \mu \delta_{i j} \nabla \cdot \mathbf{u}+2 \mu D_{i j}=-p_{0} \delta_{i j}+2 \mu\left(D_{i j}-\frac{1}{3} \nabla \cdot \mathbf{u}\right) .
$$

Equation 3.15 is the final form for a Newtonian compressible and Stokesian fluid. This form is the most common fluid model used in CFD, along with the continuity, momentum and energy equations. 


\subsubsection{The Non-Stokesian Fluid}

The non-Stokesian fluid is an even more general case that may be reduced to the Stokesian fluid. In this case, we may write $\sigma_{i j}$ in the following form of the second viscosity coefficient:

$$
\sigma_{i j}=-p_{0} \delta_{i j}+\kappa \delta_{i j} \nabla \cdot \mathbf{u}+2 \mu\left(D_{i j}-\frac{1}{3} \delta_{i j} \nabla \cdot \mathbf{u}\right),
$$

that is the constitutive equation that describes flows with dissipative effects related to compressibility, where the thermodynamic non-equilibrium effects are not negligible. At these conditions, the time of response or relaxation time of the dilating fluid are larger than the excitation time. The flow itself becomes an irreversible process with a certain deviation from the equilibrium with $\kappa \neq 0$.

For inviscid barotropic flows under high frequencies, equation 3.16 is reduced to the following form:

$$
\sigma_{i j}=-p_{0} \delta_{i j}+\kappa \delta_{i j} \nabla \cdot \mathbf{u}
$$

where $\lambda=\kappa$ in this case. Now we apply the constitutive equation 3.17 into Cauchy's equation without body forces, which is the representation of Newton's second law for a continuous media [61]:

$$
\rho \frac{D \mathbf{u}}{D t}=\nabla \cdot \sigma
$$

we then have:

$$
\rho \frac{D \mathbf{u}}{D t}=-\nabla p_{0}+\nabla(\kappa \nabla \cdot \mathbf{u})
$$

\subsubsection{Energy Considerations for the General Newtonian Stress Tensor}

From the energy equation 2.10 in its differential and conservative form for a generalized Newtonian fluid, we have:

$$
\frac{\left(\partial \rho e_{t}\right)}{\partial t}+\nabla \cdot\left(\rho \mathbf{u} e_{t}\right)=\nabla \cdot(-\mathbf{q}+\tau \cdot \mathbf{u}-p \mathbf{I} \cdot \mathbf{u})+\rho \mathbf{f}_{e} \cdot \mathbf{u}
$$

and by its non-conservative equivalent form:

$$
\rho \frac{D}{D t}\left(\frac{u^{2}}{2}+e\right)=\nabla \cdot(-\mathbf{q}+\sigma \cdot \mathbf{u})+\rho \mathbf{f}_{e} \cdot \mathbf{u},
$$


where $\sigma \cdot \mathbf{u}=\tau \cdot \mathbf{u}-p \mathbf{I} \cdot \mathbf{u}$. By defining the body forces through a potencial field, we have:

$$
\rho \mathbf{f}_{e} \cdot \mathbf{u}=-\rho \nabla \phi \cdot \mathbf{u}=-\rho \frac{D \phi}{D t}
$$

and with the following vector identity:

$$
\nabla \cdot(\sigma \cdot \mathbf{u})=\mathbf{u} \cdot \nabla \cdot \sigma+\sigma: \nabla \mathbf{u}
$$

Substituting equations 3.22 and 3.23 into equation 3.21, we have:

$$
\rho \frac{D}{D t}\left(\frac{u^{2}}{2}+e\right)=-\nabla \cdot \mathbf{q}+\mathbf{u} \cdot \nabla \cdot \sigma+\sigma: \nabla \mathbf{u}-\rho \nabla \phi \cdot \mathbf{u}
$$

or in an equivalent form:

$$
\rho \frac{D}{D t}\left(\frac{u^{2}}{2}+e+\phi\right)-\mathbf{u} \cdot \nabla \cdot \sigma=-\nabla \cdot \mathbf{q}+\sigma: \nabla \mathbf{u}
$$

In equation 3.25 it is implicit the mechanical energy equation (mathematical derivation in appendix III):

$$
\rho \frac{D}{D t}\left(\frac{u^{2}}{2}+\phi\right)=\mathbf{u} \cdot \nabla \cdot \sigma
$$

where the resulting equation is the Kirchoff's equation of internal energy:

$$
\rho \frac{D e}{D t}=-\nabla \cdot \mathbf{q}+\sigma: \nabla \mathbf{u}
$$

Now we consider the stress tensor for a non-Stokesian Newtonian fluid (equation 3.16), repeated here:

$$
\sigma=-p_{0} \mathbf{I}+\kappa \nabla \cdot \mathbf{u}+2 \mu\left(\mathbf{D}-\frac{1}{3}(\nabla \cdot \mathbf{u}) \mathbf{I}\right) .
$$

Taking into account that the above tensor is symmetric $\left(\sigma_{i j}=\sigma_{j i}\right)$, the second term of the right hand side from equation 3.27 may be split into the symmetric and antisymmetric parts:

$$
\sigma: \nabla \mathbf{u}=\sigma: \mathbf{D}+\sigma: \mathbf{W}
$$

where the antisymmetric part is equal to zero, then we have:

$$
\sigma: \nabla \mathbf{u}=\sigma: \mathbf{D}
$$

By substituting equation 3.16 into equation 3.30:

$$
\sigma: \nabla \mathbf{u}=-p_{0} \nabla \cdot \mathbf{u}+\left(\kappa-\frac{2}{3} \mu\right)(\nabla \cdot \mathbf{u})^{2}+2 \mu \mathbf{D}: \mathbf{D}
$$


Now by taking the entropic form of the second law of thermodynamics equation, we have:

$$
T d s=d e+p_{0} d\left(\frac{1}{\rho}\right)=d e-\frac{p_{0}}{\rho^{2}} d \rho .
$$

Differentiating equation 3.32 in respect to time:

$$
\rho T \frac{D s}{D t}=\rho \frac{D e}{D t}-\frac{p_{0}}{\rho} \frac{D \rho}{D t}
$$

Substituting the second term of the right hand side of equation 3.33 by its counterpart from continuity equation results in:

$$
\rho T \frac{D s}{D t}=\rho \frac{D e}{D t}+p_{0} \nabla \cdot \mathbf{u}
$$

Substituting equations 3.27 and 3.31 into equation 3.34 and using the material derivative notation:

$$
\rho \frac{D s}{D t}=\frac{1}{T}\left[-p_{0}(\nabla \cdot \mathbf{u})+\left(\kappa-\frac{2}{3} \mu\right)(\nabla \cdot \mathbf{u})^{2}+2 \mu \mathbf{D}: \mathbf{D}+p_{0}(\nabla \cdot \mathbf{u})-\nabla \cdot \mathbf{q}\right],
$$

resulting in:

$$
\rho \frac{D s}{D t}=\frac{1}{T}\left[\left(\kappa-\frac{2}{3} \mu\right)(\nabla \cdot \mathbf{u})^{2}+2 \mu \mathbf{D}: \mathbf{D}-\nabla \cdot \mathbf{q}\right]
$$

Equation 3.36 states the second law of thermodynamics under the entropic form where the right hand side terms of the equation are the entropy generation sources. The first term is the expansional viscosity effect associated with the fluid dilatation and contraction. The second term is the shear viscosity effect acting on a shearing fluid. The third term is the effect of thermal condution on the fluid.

Note that if the fluid is Stokesian, in the first term of the right hand side, expression 3.13 will be valid in equation 3.36 and it will be equal to zero and as a consequence there will not be entropy growth due to dilatation and contraction effects on the fluid.

\subsubsection{Second Viscosity Coefficient for an Inviscid Non-Stokesian Fluid}

The second viscosity coefficient may be modeled after phenomena that oscillate between the equilibrium and non-equilibrium states. The magnitude of the expansional viscosity is usually the same as the shear viscosity, but in some cases, the expansional viscosity 
magnitude may surpass the shear viscosity magnitude [20]. As shown in equation 3.17, the second viscosity coefficient is multiplied by the velocity divergent, which represents the dilatation and contraction rate per unit of volume of a fluid element. In cases where there is a fast dilatation or contraction rate of the fluid, it leaves the thermodynamic equilibrium, and then internal processes occur so that the fluid goes back to the equilibrium condition. In cases where there is acoustic propagation, the dilatation or contraction of the fluid occurs at a characteristic frequency. The second viscosity coefficient then acts with this dilatation or contraction and as a consequence, the effect of the second viscosity coefficient is directly related with not only the material but also with the flow itself, in the same way that happens with the shear viscosity.

Now we consider a given phenomenon where $\xi$ denotes an arbitrary state of the fluid from a given physical quantity, and that $\xi_{0}$ is the same physical quantity but at thermodynamic equilibrium. We also consider that like any thermodynamic quantity, $\xi$ depends on this case of the fluid temperature and density. If the fluid is not at thermodynamic equilibrium, $\xi$ will change with time towards the equilibrium value $\xi_{0}$.

We now take a Taylor series expanding $\xi_{0}$ around its equilibrium state, relative to the reference time $t_{0}$, and the same property $\xi$ but out of the equilibrium state, which is evaluated at a physical time $t$. Also consider that the relaxation time fluid, that is the time needed to go from the non-equilibrium to the equilibrium condition $\tau=t-t_{0}$, where large values for $\tau$ means that the fluid will go back to the state of equilibrium slower than small relaxation times:

$$
\xi(t)=\xi_{0}\left(t_{0}\right)-\left.\left(t-t_{0}\right) \frac{d \xi}{d t}\right|_{t=t_{0}}+O\left(\tau^{2}\right)
$$

taking only the linear terms of the Taylor series, we have:

$$
\xi(t)=\xi_{0}\left(t_{0}\right)-\tau \dot{\xi}
$$

rearranging the terms and isolating the derivative:

$$
\dot{\xi}=-\frac{\left(\xi-\xi_{0}\right)}{\tau} .
$$

Now we consider physical processes where the fluid goes under periodic and adiabatic dilatation or contraction. This implies that the density and other thermodynamic variables will also be subjected to temporal periodic changes. As a consequence, the equilibrium 
state $\xi_{0}$ may be written as:

$$
\xi_{0}=\xi_{00}+\xi_{0}^{\prime}
$$

where $\xi_{00}$ is the mean value and is non-time dependent, and $\xi_{0}^{\prime}$ is the fluctuation of the equilibrium state subjected to the periodic oscillation $e^{-i \omega t}$. By the same idea the nonequilibrium state may be defined by its fluctuating and mean variables, we then have:

$$
\xi=\xi_{00}+\xi^{\prime}
$$

By combining equations 3.40 and 3.41 with equation 3.39 , we have the non-equilibrium relaxation time derivative related to its fluctuation terms, given by:

$$
\dot{\xi}=\frac{d}{d t}\left(\xi_{00}+\xi^{\prime}\right)=-\frac{\xi_{00}+\xi^{\prime}-\xi_{00}-\xi_{0}^{\prime}}{\tau}
$$

or yet:

$$
\dot{\xi}^{\prime}=-\frac{\xi^{\prime}-\xi_{0}^{\prime}}{\tau}
$$

Equation 3.43 states that if the fluctuations are function of a certain periodic fluctuation:

$$
\begin{aligned}
\xi^{\prime} & =\hat{\xi} e^{-i \omega t} \\
\xi_{0}^{\prime} & =\hat{\xi}_{0} e^{-i \omega t}
\end{aligned}
$$

where $\hat{\xi}$ and $\hat{\xi}_{0}$ are the amplitudes of the terms in and out the thermodynamic equilibrium, respectively, for the $\xi$ fluctuation. By substituting equations 3.44 and 3.45 into equation 3.43, we have:

$$
-i \omega \hat{\xi} e^{-i \omega t}=-\frac{\hat{\xi} e^{-i \omega t}-\hat{\xi_{0}} e^{-i \omega t}}{\tau} .
$$

Arranging the terms from equation 3.46:

$$
\hat{\xi}=\frac{\hat{\xi}_{0}}{1-i \tau \omega} .
$$

Equation 3.47 may also be expressed from the mathematical point of view as the frequency domain transform, where the resulting equation is the amplitude fluctuation of the equilibrium states as a function of thermodynamic states (relaxation time $\tau$ ) and flow variables (excitation frequency $\omega$ ). The main idea here is to obtain a constitutive model for the second viscosity coefficient in the frequency domain, where it can be brought to the time 
domain. By using the Fourier transform property for derivatives [62], we may define the following operators of their own domain:

$$
\begin{aligned}
-i \omega & \leftrightarrow \frac{\partial}{\partial t}, \text { order } 1 \\
\omega^{2} & \leftrightarrow \frac{\partial^{2}}{\partial t^{2}}, \text { order } 2 \\
\vdots & \\
(-i \omega)^{n} & \leftrightarrow \frac{\partial^{n}}{\partial t^{n}}, \text { order } \mathrm{n} .
\end{aligned}
$$

Consider now the total derivative of the pressure in respect to the fluid density and the thermodynamic property $\xi$, where $p=p(\rho, \xi(\rho))$, we then have:

$$
\frac{d p}{d \rho}=\left(\frac{\partial p}{\partial \rho}\right)_{\xi}+\left(\frac{\partial p}{\partial \xi}\right)_{\rho} \frac{\partial \xi}{\partial \rho} .
$$

Substituting equation 3.47 on the second term of the right hand side of equation 3.49 results in:

$$
\frac{\partial \xi}{\partial \rho}=\frac{\partial}{\partial \rho}\left(\frac{\hat{\xi_{0}}}{1-i \omega \tau}\right)=\frac{1}{1-i \omega \tau} \frac{\partial \hat{\xi}_{0}}{\partial \rho}
$$

substituting equation 3.50 into equation 3.49:

$$
\frac{d p}{d \rho}=\left(\frac{\partial p}{\partial \rho}\right)_{\xi}+\frac{1}{1-i \omega \tau}\left(\frac{\partial p}{\partial \xi}\right)_{\rho} \frac{\partial \hat{\xi}_{0}}{\partial \rho} .
$$

Equation 3.51 may also have the following form:

$$
\frac{d p}{d \rho}=\frac{1}{1-i \omega \tau}\left[\left(\frac{\partial p}{\partial \rho}\right)_{\xi}+\left(\frac{\partial p}{\partial \xi}\right)_{\rho} \frac{\partial \hat{\xi}_{0}}{\partial \rho}-i \omega \tau\left(\frac{\partial p}{\partial \rho}\right)_{\xi}\right] .
$$

The first two terms of the right hand side of equation 3.52 are the total derivative of the pressure for physical processes that are slow enough so that they are in thermodynamic equilibrium:

$$
\frac{d p}{d \rho}=\frac{1}{1-i \omega \tau}\left[\left(\frac{d p}{d \rho}\right)_{e q}-i \omega \tau\left(\frac{\partial p}{\partial \rho}\right)_{\xi}\right] .
$$

On equation 3.53, we have two distinct conditions for the pressure $p$. The first one is the pressure at the thermodynamic equilibrium state $p_{0}$ and the second one the pressure out of the thermodynamic equilibrium. The pressure at the equilibrium $p_{0}$ is easily obtained by the usual equation of state (equation 2.13), which may be entirely defined, for example, when density and entropy are known variables. The pressure in non-equilibrium or mechanical 
pressure is different from $p_{0}$, being a function of variables also outside the equilibrium state $(\hat{\xi})$.

We consider now a change of $\delta \rho$ on the fluid. As a consequence, we have the following changes for the equilibrium and non-equilibrium states:

- Equilibrium state:

$$
\delta p_{0}=\left(\frac{d p}{d \rho}\right)_{e q} \delta \rho .
$$

- Non equilibrium state by combining equations 3.54 and 3.52 :

$$
\delta p=\left(\frac{d p}{d \rho}\right) \delta \rho=\left(\frac{1}{1-i \omega \tau}\left[\left(\frac{d p}{d \rho}\right)_{e q}-i \omega \tau\left(\frac{\partial p}{\partial \rho}\right)_{\xi}\right]\right) \delta \rho .
$$

We may also define the pressure deviation outside the equilibrium state (mechanical pressure) as a reference to the thermodynamic pressure, function of the variation of the thermodynamic state of $\rho+\delta \rho$ :

$$
\delta p-\delta p_{0}=\left[\frac{d p}{d \rho}-\left(\frac{d p}{d \rho}\right)_{e q}\right] \delta \rho .
$$

Substituting equation 3.55 into equation 3.56, we have:

$$
\begin{aligned}
\delta p-\delta p_{0} & =\left[\frac{1}{1-i \omega \tau}\left[\left(\frac{d p}{d \rho}\right)_{e q}-i \omega \tau\left(\frac{\partial p}{\partial \rho}\right)_{\xi}\right]-\left(\frac{d p}{d \rho}\right)_{e q}\right] \delta \rho, \\
& =\frac{i \omega \tau}{1-i \omega \tau}\left[\left(\frac{d p}{d \rho}\right)_{e q}-\left(\frac{\partial p}{\partial \rho}\right)_{\xi}\right] \delta \rho .
\end{aligned}
$$

The continuity equation may be given by the following form:

$$
\frac{D}{D t}(\delta \rho)=-\rho \nabla \cdot \mathbf{u}
$$

The above expression represents the rate of volume change per unit of time of a given fluid element $\delta \rho$. We now apply the Fourier transform on equation 3.58:

$$
i \omega \delta \rho=\rho \nabla \cdot \mathbf{u}
$$

Now, isolating the term $\delta \rho$ of equation 3.59 we have:

$$
\delta \rho=\left(\frac{\rho}{i \omega}\right) \nabla \cdot \mathbf{u} .
$$


By substituting equation 3.60 into equation 3.55, we obtain:

$$
\delta p-\delta p_{0}=\frac{\rho \tau}{1-i \omega \tau}\left[\left(\frac{\partial p}{\partial \rho}\right)_{e q}-\left(\frac{\partial p}{\partial \rho}\right)_{\xi}\right] \nabla \cdot \mathbf{u} .
$$

Also, note that:

$$
\delta p-\delta p_{0}=\left(p-p_{\infty}\right)-\left(p_{0}-p_{\infty}\right)=p-p_{0}
$$

For a barotropic fluid $(p=p(\rho))$, the speed of sound at equilibrium and at nonequilibrium are:

$$
\begin{aligned}
& \left(\frac{\partial p}{\partial \rho}\right)_{e q}=c_{0}^{2} \\
& \left(\frac{\partial p}{\partial \rho}\right)_{\xi}=c_{\infty}^{2} .
\end{aligned}
$$

Substituting equations 3.63 and 3.64 into equation 3.61 we have:

$$
-\left(p-p_{0}\right)=\frac{\rho \tau}{1-i \omega \tau}\left(c_{\infty}^{2}-c_{0}^{2}\right) \nabla \cdot \mathbf{u}
$$

By comparing equation 3.65 with the constitutive equation 3.11, which defines the mechanical pressure for a compressible fluid under rapid dilatation and contraction, we obtain an equation for the second viscosity coefficient, or expansional viscosity, given by:

$$
\kappa=\frac{\rho \tau}{1-i \omega \tau}\left(c_{\infty}^{2}-c_{0}^{2}\right)
$$

or in an alternative form:

$$
\kappa=\frac{\rho \tau c_{0}^{2}}{1-i \omega \tau}\left(r_{M}^{2}-1\right),
$$

where the term $r_{M}$ is then defined as:

$$
r_{M}=\frac{c_{\infty}}{c_{0}}=\frac{U_{0}}{c_{0}} \frac{c_{\infty}}{U_{0}}=\frac{M_{0}}{M_{\infty}}
$$

it is also a valid interpretation of the ratio of Mach numbers that are related to the thermodynamic equilibrium and non-equilibrium.

The momentum equation with the derived model of the second viscosity coefficient is given by:

$$
\rho\left(\frac{\partial \mathbf{u}}{\partial t}+\mathbf{u} \cdot \nabla \mathbf{u}\right)=-\nabla p_{0}+\nabla(\kappa \nabla \cdot \mathbf{u})
$$


For the closure of the system of equations we will use the continuity equation and a general barotropic relation for the pressure in thermodynamic equilibrium:

$$
p_{0}=\beta \rho^{\gamma}
$$

where $\beta$ and $\gamma$ are material constants of the fluid.

By organizing the governing equations and their closure expressions, we have:

- Continuity equation:

$$
\frac{\partial \rho}{\partial t}+\rho \nabla \cdot \mathbf{u}+\mathbf{u} \cdot \nabla \rho=0
$$

- Linear momentum equation:

$$
\rho\left(\frac{\partial \mathbf{u}}{\partial t}+\mathbf{u} \cdot \nabla \mathbf{u}\right)+\nabla p_{0}-\nabla(\kappa \nabla \cdot \mathbf{u})=0
$$

- Expansional viscosity equation in frequency domain:

$$
\kappa=\frac{\rho \tau c_{0}^{2}}{1-i \omega \tau}\left(r_{M}^{2}-1\right) .
$$

- Barotropic equation:

$$
p_{0}(\rho)=\beta \rho^{\gamma} .
$$

\subsection{Linear Stability Analisys of the Governing Equations}

The system of governing equations obtained in section 3.1.5 (equations 3.71 to 3.74 ), is composed of nonlinear partial differential equations and in most cases, their solution is nonexistent or may not satisfy the boundary conditions. A numerical methodology is needed for approximate solutions. Before the proposal of a numerical methodology to solve the system of governing equations, a linear stability analysis will be carried out by introducing perturbations into the linearized governing equations.

\subsubsection{Non-Dimensional Form of the Governing Equations}

In order to identify the non-dimensional parameters that represent the physical phenomena of the governing equations, we propose the following expressions to obtain the 
non-dimensional form of the governing equations, where the superscript ${ }^{*}$ indicates a nondimensional value:

$$
x^{*}=\frac{x}{L_{0}}, \quad u^{*}=\frac{u}{U_{0}}, \quad t^{*}=\frac{t}{L_{0} / U_{0}}, \quad \rho^{*}=\frac{\rho}{\rho_{0}}, \quad p^{*}=\frac{p}{\rho_{0}\left(U_{0}\right)^{2}},
$$

where the variables with subscript 0 are reference values that are on thermodynamic equilibrium. From this point on the governing equations we will assume the one dimensional unsteady form: $\rho=\rho(x, t), u=u(x, t)$ e $p=p(x, t)$.

By substituting equations 3.75 into the continuity equation 3.71, we have:

$$
\left(\frac{U_{0} \rho_{0}}{L_{0}}\right) \frac{\partial \rho^{*}}{\partial t^{*}}+\left(\frac{U_{0} \rho_{0}}{L_{0}}\right) \rho^{*} \frac{\partial u^{*}}{\partial x^{*}}+\left(\frac{U_{0} \rho_{0}}{L_{0}}\right) u^{*} \frac{\partial \rho^{*}}{\partial x^{*}}=0
$$

that is equivalent to the dimensional form, after cancelling any non-dimensional terms:

$$
\frac{\partial \rho^{*}}{\partial t^{*}}+\rho^{*} \frac{\partial u^{*}}{\partial x^{*}}+u^{*} \frac{\partial \rho^{*}}{\partial x^{*}}=0
$$

Consider the one dimensional form of equation 3.72 :

$$
\frac{\partial u}{\partial t}+u \frac{\partial u}{\partial x}+\frac{1}{\rho} \frac{\partial p}{\partial x}-\frac{1}{\rho} \frac{\partial}{\partial x}\left(\kappa(\rho) \frac{\partial u}{\partial x}\right)=0
$$

Opening the third term from the left hand side of equation 3.78 and applying the chain rule for $\kappa(\rho)$, we have:

$$
\frac{\partial u}{\partial t}+u \frac{\partial u}{\partial x}+\frac{1}{\rho} \frac{\partial p}{\partial x}-\frac{1}{\rho} \frac{\partial \kappa}{\partial \rho} \frac{\partial \rho}{\partial x} \frac{\partial u}{\partial x}-\frac{\kappa}{\rho} \frac{\partial^{2} u}{\partial u^{2}}=0
$$

Substituing equation 3.73 into equation 3.79 , we then have:

$$
\frac{\partial u}{\partial \rho}+u \frac{\partial u}{\partial x}+\frac{1}{\rho} \frac{\partial p_{0}}{\partial x}-\left[\frac{\tau c_{0}^{2}\left(r_{M}^{2}-1\right)}{1-i \omega \tau}\right] \frac{\partial^{2} u}{\partial x^{2}}-\frac{1}{\rho}\left[\frac{\tau c_{0}^{2}\left(r_{M}^{2}-1\right)}{1-i \omega \tau}\right] \frac{\partial \rho}{\partial x} \frac{\partial u}{\partial x}=0
$$

Substituting the non-dimensional expressions 3.75 into equation 3.80 and rearranging:

$$
\frac{\partial u^{*}}{\partial t^{*}}+u^{*} \frac{\partial u^{*}}{\partial x^{*}}+\frac{1}{\rho^{*}} \frac{\partial p_{0}^{*}}{\partial x^{*}}-\left[\frac{\tau c_{0}^{2}}{U_{0} L_{0}}\right]\left(\frac{\left(r_{M}^{2}-1\right)}{1-i \omega \tau}\right) \frac{\partial u^{2}}{\partial x^{2}}-\frac{1}{\rho^{*}}\left[\frac{\tau c_{0}^{2}}{U_{0} L_{0}}\right]\left(\frac{\left(r_{M}^{2}-1\right)}{1-i \omega \tau}\right) \frac{\partial \rho^{*}}{\partial x^{*}} \frac{\partial u^{*}}{\partial x^{*}}=0 .
$$

The term between brackets on equation 3.81 may be identified as the Reynolds number of the expansional viscosity:

$$
R e_{\kappa}=\frac{L_{0} U_{0}}{\tau c_{0}^{2}}=\frac{L_{0} M_{0}}{\tau c_{0}}=\frac{\left(\frac{L_{0}}{U_{0}}\right)}{\tau} \frac{U_{0}^{2}}{c_{0}^{2}}=\frac{t_{0} M_{0}^{2}}{\tau},
$$


where $M_{0}$ is the Mach number based on the reference or equilibrium velocity:

$$
M_{0}=\frac{U_{0}}{c_{0}}
$$

The given interpretation for the non-dimensional group 3.82 is the mathematical relation between the effects of equilibrium $\left(U_{0}\right)$ and non-equilibrium $(\tau)$, directly associated with compressibility of the flow $\left(M_{0}\right)$ and the characteristic length of the flow $\left(L_{0}\right)$. Another interpretation that may be given is the ratio of the characteristic times of the flow in equilibrium $\left(t_{0}\right)$, the compressibility effects and the fluid relaxation time $(\tau)$. If the compressibility or the flow effects have a larger time than the effects in non-equilibrium, the momentum dissipation due to the fluid dilatation and contraction will happen much faster and its effects will be negligible. If the time associated with the dissipation due to the expansional viscosity is larger than the flow characteristic time or compressibility effects, there will be a larger influence of the expansional viscosity and its dissipation effects will not be negligible.

Substituting equation 3.82 into equation 3.81 we have:

$$
\frac{\partial u^{*}}{\partial t^{*}}+u^{*} \frac{\partial u^{*}}{\partial x^{*}}+\frac{1}{\rho^{*}} \frac{\partial p_{0}^{*}}{\partial x^{*}}-\frac{1}{R e_{\kappa}}\left(\frac{\left(r_{M}^{2}-1\right)}{1-i \omega \tau}\right) \frac{\partial u^{2}}{\partial x^{2}}-\frac{1}{\rho^{*} R e_{\kappa}}\left(\frac{\left(r_{M}^{2}-1\right)}{1-i \omega \tau}\right) \frac{\partial \rho^{*}}{\partial x^{*}} \frac{\partial u^{*}}{\partial x^{*}}=0 .
$$

Now applying the chain rule on the third term of equation 3.84 and according to the barotropic equation 3.74 , where $p_{0}^{*}=p_{0}^{*}\left(\rho^{*}\left(x^{*}\right)\right)$ :

$$
\frac{\partial p_{0}^{*}}{\partial x^{*}}=\frac{\partial p_{0}^{*}}{\partial \rho^{*}} \frac{\partial \rho^{*}}{\partial x^{*}} .
$$

The first derivative on the right hand side of equation 3.85 may be evaluated in the barotropic equation 3.74 in its non-dimensional form:

$$
\frac{\partial p_{0}^{*}}{\partial \rho^{*}}=\beta^{*} \gamma^{*} \rho^{\gamma^{*}-1}=\beta^{*} \gamma^{*} \frac{\rho^{* \gamma^{*}}}{\rho^{*}}=\gamma^{*} \frac{p_{0}^{*}}{\rho^{*}}
$$

By substituting equation 3.86 into equation 3.84, we have the following nonlinear form of the momentum equation:

$$
\frac{\partial u^{*}}{\partial t^{*}}+u^{*} \frac{\partial u^{*}}{\partial x^{*}}+\gamma^{*} \frac{p_{0}^{*}}{\rho^{* 2}} \frac{\partial \rho^{*}}{\partial x^{*}}-\frac{1}{R e_{\kappa}}\left(\frac{\left(r_{M}^{2}-1\right)}{1-i \omega \tau}\right)\left(\frac{\partial^{2} u^{*}}{\partial x^{* 2}}+\frac{\partial \rho^{*}}{\partial x^{*}} \frac{\partial u^{*}}{\partial x^{*}}\right)=0
$$

The non-dimensional and final form of the system of governing equations is given by (suppressing the superscript ${ }^{*}$ from now on): 
- Continuity equation:

$$
\frac{\partial \rho}{\partial t}+\rho \frac{\partial u}{\partial x}+u \frac{\partial \rho}{\partial x}=0
$$

- Momentum equation:

$$
\frac{\partial u}{\partial t}+u \frac{\partial u}{\partial x}+\gamma \frac{p_{0}}{\rho^{2}} \frac{\partial \rho}{\partial x}-\frac{1}{R e_{\kappa}}\left(\frac{\left(r_{M}^{2}-1\right)}{1-i \omega \tau}\right)\left(\frac{\partial^{2} u}{\partial x^{2}}+\frac{\partial \rho}{\partial x} \frac{\partial u}{\partial x}\right)=0 .
$$

- Barotropic equation:

$$
p_{0}=\beta \rho^{\gamma},
$$

resulting in a system of three equations with their non-dimensional groups and three unknown variables: $u, p_{0}$ and $\rho$.

\subsubsection{Linear Form of the Governing Equations}

The decomposition into mean (equilibrium) values and fluctuations of non-equilibrium of any flow variable is given by:

$$
f=f_{0}+f^{\prime}
$$

In the linear stability analysis context, the fluctuation terms $f^{\prime}$ are much smaller than their equilibrium or mean counterparts, i.e., $\left|f^{\prime}\right| /\left|f_{0}\right| \ll 1$. As a consequence, the second order or superior fluctuation terms $\left(f^{\prime 2}\right)$ will be neglected in this analysis. In this case the mean or equilibrium values will be regarded as constant in space and time permanent. By applying the decomposition 3.91 for the density and velocity variables $\rho$ e $u$, we have:

$$
\begin{aligned}
& \rho(x, t)=1+\rho^{\prime}(x, t), \\
& u(x, t)=1+u^{\prime}(x, t) .
\end{aligned}
$$

Now applying the decompositions 3.92 and 3.93 into the continuity equation 3.88 :

$$
\frac{\partial}{\partial t}\left(1+\rho^{\prime}\right)+\left(1+\rho^{\prime}\right) \frac{\partial}{\partial x}\left(1+u^{\prime}\right)+\left(1+u^{\prime}\right) \frac{\partial}{\partial x}\left(1+\rho^{\prime}\right)=0 .
$$

Developing equation 3.94 and neglecting the superior order terms of fluctuation:

$$
\frac{\partial \rho^{\prime}}{\partial t}+\frac{\partial u^{\prime}}{\partial x}+\frac{\partial \rho^{\prime}}{\partial x}=0
$$


Applying the density fluctuation equation 3.92 into the baratropic equation 3.90, we have:

$$
p_{0}=\beta \rho^{\gamma}=\beta\left(1+\rho^{\prime}\right)^{\gamma} .
$$

Equation 3.96 may be put in a linear form from Newton's binomial series:

$$
(1+x)^{n}=1+n x+\frac{n(n-1)}{2 !} x^{2}+O\left(x^{3}\right),
$$

where the neglected arguments are the second order $O\left(x^{2}\right)$ and higher terms. By combining equation 3.97 with equation 3.96 we have:

$$
\beta\left(1+\rho^{\prime}\right)^{\gamma} \cong\left(1+\gamma \rho^{\prime}\right) \beta
$$

By applying the velocity perturbation 3.92 and density 3.93 terms and the linearized barotropic equation 3.98 into the momentum equation 3.89 we have:

$$
\begin{aligned}
\frac{\partial}{\partial t}\left(1+u^{\prime}\right)+(1 & \left.+u^{\prime}\right) \frac{\partial}{\partial x}\left(1+u^{\prime}\right)+\gamma \beta \frac{\left(1+\gamma \rho^{\prime}\right)}{\left(1+\rho^{\prime}\right)^{2}} \frac{\partial}{\partial x}\left(1+\rho^{\prime}\right) \\
& -\frac{1}{R e_{\kappa}}\left(\frac{\left(r_{M}^{2}-1\right)}{1-i \omega \tau}\right)\left(\frac{\partial^{2}}{\partial x^{2}}\left(1+u^{\prime}\right)+\frac{\partial}{\partial x}\left(1+\rho^{\prime}\right) \frac{\partial}{\partial x}\left(1+u^{\prime}\right)\right)=0 .
\end{aligned}
$$

Now evaluating the derivatives at their respective perturbation terms and neglecting again the fluctuation terms of higher order:

$$
\frac{\partial u^{\prime}}{\partial t}+\frac{\partial u^{\prime}}{\partial x}+\frac{\gamma \beta}{\left(1+\rho^{\prime}\right)^{2}} \frac{\partial \rho^{\prime}}{\partial x}-\frac{1}{R e_{\kappa}}\left(\frac{\left(r_{M}^{2}-1\right)}{1-i \omega \tau}\right) \frac{\partial^{2} u^{\prime}}{\partial x^{2}}=0
$$

Expanding the denominator of the third term from equation 3.100 by using the Newton's binomial series and neglecting the higher order perturbation terms:

$$
\left(1+\rho^{\prime}\right)^{-2} \cong 1-2 \rho^{\prime}
$$

By substituting equation 3.101 into equation 3.100 and again neglecting the superior order perturbation terms, we have:

$$
\frac{\partial u^{\prime}}{\partial t}+\frac{\partial u^{\prime}}{\partial x}+\gamma \beta \frac{\partial \rho^{\prime}}{\partial x}-\frac{1}{R e_{\kappa}}\left(\frac{\left(r_{M}^{2}-1\right)}{1-i \omega \tau}\right) \frac{\partial^{2} u^{\prime}}{\partial x^{2}}=0 .
$$

The linearized non-dimensional form of governing equations system is finally given by:

- Continuity equation:

$$
\frac{\partial \rho^{\prime}}{\partial t}+\frac{\partial u^{\prime}}{\partial x}+\frac{\partial \rho^{\prime}}{\partial x}=0
$$

- Momentum equation:

$$
\frac{\partial u^{\prime}}{\partial t}+\frac{\partial u^{\prime}}{\partial x}+\gamma \beta \frac{\partial \rho^{\prime}}{\partial x}-\frac{1}{R e_{\kappa}}\left(\frac{\left(r_{M}^{2}-1\right)}{1-i \omega \tau}\right) \frac{\partial^{2} u^{\prime}}{\partial x^{2}}=0 .
$$




\subsubsection{The Oscillatory Nature of the Linearized Governing Equations}

By applying the quiescent fluid condition to equations 3.103 and 3.104, the derivatives $\partial \rho^{\prime} / \partial x$ and $\partial u^{\prime} / \partial x$ will be equal to zero in continuity equation 3.103 and momentum equation 3.104, respectively:

- Continuity equation:

$$
\frac{\partial \rho^{\prime}}{\partial t}+\frac{\partial u^{\prime}}{\partial x}=0
$$

- Momentum equation:

$$
\frac{\partial u^{\prime}}{\partial t}+\gamma \beta \frac{\partial \rho^{\prime}}{\partial x}-\frac{1}{R e_{\kappa}}\left(\frac{\left(r_{M}^{2}-1\right)}{1-i \omega \tau}\right) \frac{\partial^{2} u^{\prime}}{\partial x^{2}}=0
$$

Differentiating equation 3.105 in respect to time and equation 3.106 in respect to space coordinate, we have:

- Continuity equation:

$$
\frac{\partial^{2} \rho^{\prime}}{\partial t^{2}}+\frac{\partial^{2} u^{\prime}}{\partial t \partial x}=0
$$

- Momentum equation:

$$
\frac{\partial^{2} u^{\prime}}{\partial x \partial t}+\gamma \beta \frac{\partial^{2} \rho^{\prime}}{\partial x^{2}}-\frac{1}{R e_{\kappa}}\left(\frac{\left(r_{M}^{2}-1\right)}{1-i \omega \tau}\right) \frac{\partial^{3} u^{\prime}}{\partial x^{3}}=0 .
$$

Subtracting equation 3.108 from equation 3.107 and rearranging we have:

$$
\frac{\partial^{2} \rho^{\prime}}{\partial t^{2}}-\gamma \beta \frac{\partial^{2} \rho^{\prime}}{\partial x^{2}}=-\frac{1}{R e_{\kappa}}\left(\frac{\left(r_{M}^{2}-1\right)}{1-i \omega \tau}\right) \frac{\partial^{3} u^{\prime}}{\partial x^{3}}
$$

Mathematically equation 3.109 is of the same oscillatory nature as the wave equation. Obtained for the perturbations outside thermodynamic equilibrium and with the stated simplifications, equation 3.109 has its own characteristics that differ from the traditional wave equation; the term related to the linearized propagation speed of the perturbations $(\gamma \beta)$ and the dissipation and dispersion associated with the expansional viscosity on the right hand side. 


\subsubsection{Infinitesimal Perturbations and Its Modal Temporal Stability}

Regarding the linearized governing equations in the non-dimensional form, there are some conditions where its behavior concerning the amplification or attenuation of the oscillatory solution can be analyzed. This analysis is useful to predict the type of waves that will have its amplitude amplified or attenuated, as well as its dispersion effects on the wave number.

\subsubsection{The Perturbations}

The stability analysis is then applied on the system of linearized governing equations:

- Continuity equation:

$$
\frac{\partial \rho^{\prime}}{\partial t}+\frac{\partial u^{\prime}}{\partial x}+\frac{\partial \rho^{\prime}}{\partial x}=0
$$

- Momentum equation:

$$
\frac{\partial u^{\prime}}{\partial t}+\frac{\partial u^{\prime}}{\partial x}+\gamma \beta \frac{\partial \rho^{\prime}}{\partial x}-\frac{1}{R e_{\kappa}}\left(\frac{\left(r_{M}^{2}-1\right)}{1-i \omega \tau}\right) \frac{\partial^{2} u^{\prime}}{\partial x^{2}}=0 .
$$

The above system of equations is then defined by two equations and two unknown variables. We consider a priori an oscillatory solution for the density and velocity:

$$
\begin{gathered}
u^{\prime}=u_{a} e^{s t+i k x}, \\
\rho^{\prime}=\rho_{a} e^{s t+i k x},
\end{gathered}
$$

where $k$ is the wave number of the $x$ coordinate and $s$ is the exponential argument of the time solution, that may be complex or imaginary. By applying this kind of solution implies the modal temporal analysis [80], where its functionality will be explained later on.

Substituting the solutions 3.112 in the continuity equation 3.110 we have:

$$
\rho_{a} e^{s t+i k x} s+\rho_{a} e^{s t+i k x} i k+u_{a} e^{s t+i k x} i k=0,
$$

arranging its terms, we then have:

$$
u_{a}=-\frac{\rho_{a}(s+i k)}{i k}
$$


Equation 3.114 expresses the relation between the density and velocity fluctuations.

Now by substituting equations 3.112 into equation of momentum 3.111 we obtain:

$$
u_{a} e^{s t+i k x} s+u_{a} e^{s t+i k x} i k+\gamma \beta \rho_{a} e^{s t+i k x} i k-\frac{1}{R e_{\kappa}}\left(\frac{\left(r_{M}^{2}-1\right)}{1-i \omega \tau}\right) u_{a} e^{s t+i k x}(i k)^{2}=0,
$$

arranging the terms again:

$$
u_{a}\left(s+i k+\frac{k^{2}}{R e_{\kappa}}\left(\frac{\left(r_{M}^{2}-1\right)}{1-i \omega \tau}\right)\right)+\rho_{a} \gamma \beta i k=0 .
$$

By substituting equation 3.114 into equation 3.116:

$$
\left(-\frac{\rho_{a}(s+i k)}{i k}\right)\left(s+i k+\frac{k^{2}}{R e_{\kappa}}\left(\frac{\left(r_{M}^{2}-1\right)}{1-i \omega \tau}\right)\right)+\rho_{a} \gamma \beta i k=0,
$$

and now arranging its terms:

$$
(s+i k)\left(s+i k+\frac{k^{2}}{R e_{\kappa}}\left(\frac{\left(r_{M}^{2}-1\right)}{1-i \omega \tau}\right)\right)+\gamma \beta k^{2}=0 .
$$

Manipulating equation 3.118 into terms of $s$ :

$$
s^{2}+s\left(i 2 k+\frac{k^{2}}{R e_{\kappa}}\left(\frac{\left(r_{M}^{2}-1\right)}{1-i \omega \tau}\right)\right)+\left(i \frac{k^{3}}{R e_{\kappa}}\left(\frac{\left(r_{M}^{2}-1\right)}{1-i \omega \tau}\right)-k^{2}+\gamma \beta k^{2}\right)=0 .
$$

\subsubsection{Temporal Modal Analysis}

Equation 3.119 has a quadratic complex solution that will give the eigenvalues $s$ and its dispersion relation for the infinitesimal disturbances as a function of the wave number $k$. As said before, the time angular frequency $s(k)$ was defined as a complex function:

$$
s(k)=\eta(k)-i \Omega(k),
$$

where $\eta(k)$ is defined as the real part and $\Omega$ is the imaginary part of $s(k)$. We will use the negative exponential time argument and the other exponential terms as positive for the sign convention.

Similar to the oscillation spatial functions, the imaginary part of $s(k)$ represents the wave oscillation on the time coordinate, where its real part $\eta$ will define the amplitude modification of the wave according to its physical parameters, the temporal modal stability analysis. Such amplitude change may be amplification if the exponential argument is positive and attenuation of the argument is negative. If a given wave with wave number $k$ 
has amplification on its amplitude with time, the wave is unstable. For the opposite case, if the wave with a given wave number $k$ attenuates its amplitude with time, the wave is stable. A critical value is the case where there is not any change of the wave amplitude in time, i.e., the amplification factor $\eta(k)$ is equal zero. We then define the mode $k$ stability:

$$
e^{[\eta(k)-i \Omega(k)] t+i k x}=\left\{\begin{array}{l}
\text { stable if } \eta(k)<0, \\
\text { critical value (stable) if } \eta(k)=0, \\
\text { unstable if } \eta(k)>0 .
\end{array}\right.
$$

In a similar way, the imaginary part of the exponential argument from equation 3.121 is directly associated with the phase speed of the perturbations, that may by given by the differentiation in respect to time of this term [66] [80], for a constant point in the wave:

$$
\Omega(k) t-k x=\text { constant }
$$

as a consequence:

$$
V(k)=\frac{d x}{d t}=\frac{\Omega(k)}{k} .
$$

Equation 3.119 has analytical solution for $\eta$ due to its quadratic nature. By Bhaskara's quadratic solution:

$$
s=\frac{-b \pm \sqrt{b^{2}-4 a c}}{2 a}
$$

where:

$$
\begin{aligned}
& a=1, \\
& b=i 2 k+\frac{k^{2}}{R e_{\kappa}}\left(\frac{\left(r_{M}^{2}-1\right)}{1-i \omega \tau}\right), \\
& c=i \frac{k^{3}}{R e_{\kappa}}\left(\frac{\left(r_{M}^{2}-1\right)}{1-i \omega \tau}\right)-k^{2}+\gamma \beta k^{2} .
\end{aligned}
$$

Note that the quadratic solution is composed of two solutions; one with a positive sign for the square root argument of equation 3.124 and the other with a negative sign. For both cases the amplification factor $\eta$ will be analyzed, given by the real part of these solutions. A physical interpretation will be given for the physical parameters of the expansional viscosity. By substituting the terms of equation 3.125 into equation 3.124 we then obtain the following 
solutions:

$$
\begin{aligned}
s_{1}= & \frac{k\left(-i k r_{M}^{2}-2 i R e_{\kappa} \omega \tau+i k+2 R e_{\kappa}\right)}{2 R e_{\kappa}(i+\omega \tau)} \\
& +\frac{\left(k \sqrt{-k^{2} r_{M}^{4}+2 k^{2} r_{M}^{2}+4 R e_{\kappa}^{2} \gamma \beta-8 i R e_{\kappa}^{2} \gamma \beta \omega \tau-4 R e_{\kappa}^{2} \gamma \beta \omega^{2} \tau^{2}-k^{2}}\right)}{2 R e_{\kappa}(i+\omega \tau)}, \\
& s_{2}=\frac{k\left(-i k r_{M}^{2}-2 i R e_{\kappa} \omega \tau+i k+2 R e_{\kappa}\right)}{2 R e_{\kappa}(i+\omega \tau)} \\
& -\frac{\left(k \sqrt{-k^{2} r_{M}^{4}+2 k^{2} r_{M}^{2}+4 R e_{\kappa}^{2} \gamma \beta-8 i R e_{\kappa}^{2} \gamma \beta \omega \tau-4 R e_{\kappa}^{2} \gamma \beta \omega^{2} \tau^{2}-k^{2}}\right)}{2 R e_{\kappa}(i+\omega \tau)} .
\end{aligned}
$$

By the amplification factor definition from equation 3.120, we may define the $\eta$ function from the real parts from equations 3.126 and 3.127 :

$$
\begin{aligned}
& \eta_{1}=\operatorname{Re}\left(s_{1}\right) \\
& \eta_{2}=\operatorname{Re}\left(s_{2}\right)
\end{aligned}
$$

As a consequence, the linear combination of the two above solutions is the general solution:

$$
\eta(k)=\eta_{1}(k)+\eta_{2}(k) .
$$

Equation 3.130 has several physical parameters to be evaluated on the linear stability analysis of the amplification factor. Typical values for these parameters that are physically consistent for the expansional viscosity model are:

- Expansional Reynolds Number:

$$
0<R e_{\kappa}<+\infty
$$

- Ratio of speeds $r_{M}$ :

$$
1<r_{M}<+\infty
$$

- Product of the oscillation frequency and the relaxation time:

$$
0<\omega \tau<+\infty
$$

- Space wave number $k$ :

$$
0<k<+\infty
$$


- Product of the material constants from the barotropic equation:

$$
0<\gamma \beta<+\infty
$$

The allowed values for the physical parameters will be the ones physically reasonable. The ratio of speeds (equation 3.132) must be larger than one. The product of the time oscillation frequency with the relaxation time (equation 3.133) may be of any positive value, where we will consider values near unity. The expansional Reynolds number (equation 3.131), similar to the wave number (equation 3.134) may have any positive value. The product of the material constants of the barotropic equation (equation 3.135) must be near unity.

Figure 3.1 shows that when $\kappa$ is equal to zero, there is no decay nor amplification for any value of wave number. As a consequence of the linearization process, there is only transport of the fluctuations out of the thermodynamic equilibrium. The positive sign solution from equation 3.130 has a behavior where the amplification factor decays indefinitely with larger values of $k$. The negative sign solution from equation 3.130 , as $k$ grows to infinity, the amplification factor decays until an asymptotic value is reached.

Figure 3.2 shows the sensitivity of the product between the oscillation $\omega$ and the thermodynamic relaxation time $\tau$. The effect of this parameter on the expansional viscosity is that, for small wave numbers, the amplification factor is larger if $\omega \tau$ has small values. For the positive sign solution the amplification factor decays indefinitely as $k$ increases. For the negative sign solution, the behavior of the amplification factor is of fast decay until a certain value for wave number. For larger values of $k$ the amplification factor tends to an asymptotic value as $k$ tends to infinity.

Figure 3.3 shows the same behavior as figure 3.2; as the expansional Reynolds number gets smaller, the amplification factor gets larger for small wave number values for all shown $R e_{\kappa}$. The same behavior is observed for all $k$ for the positive sign solution, but the opposite happens with large $k$ values for the negative sign solution. In this case there is physical incoherence, as the expansional viscosity effects become more negligible with the Reylnods number, the larger the wave amplitude attenuation becomes.

Figure 3.4 shows that for the parameter $r_{M}$ the same sensitivity can be observed from the previous cases. For small values of wave number $k$ the same behavior is observed for both solutions from equation 3.130. For the negative sign solution, for larger values of $k$, 

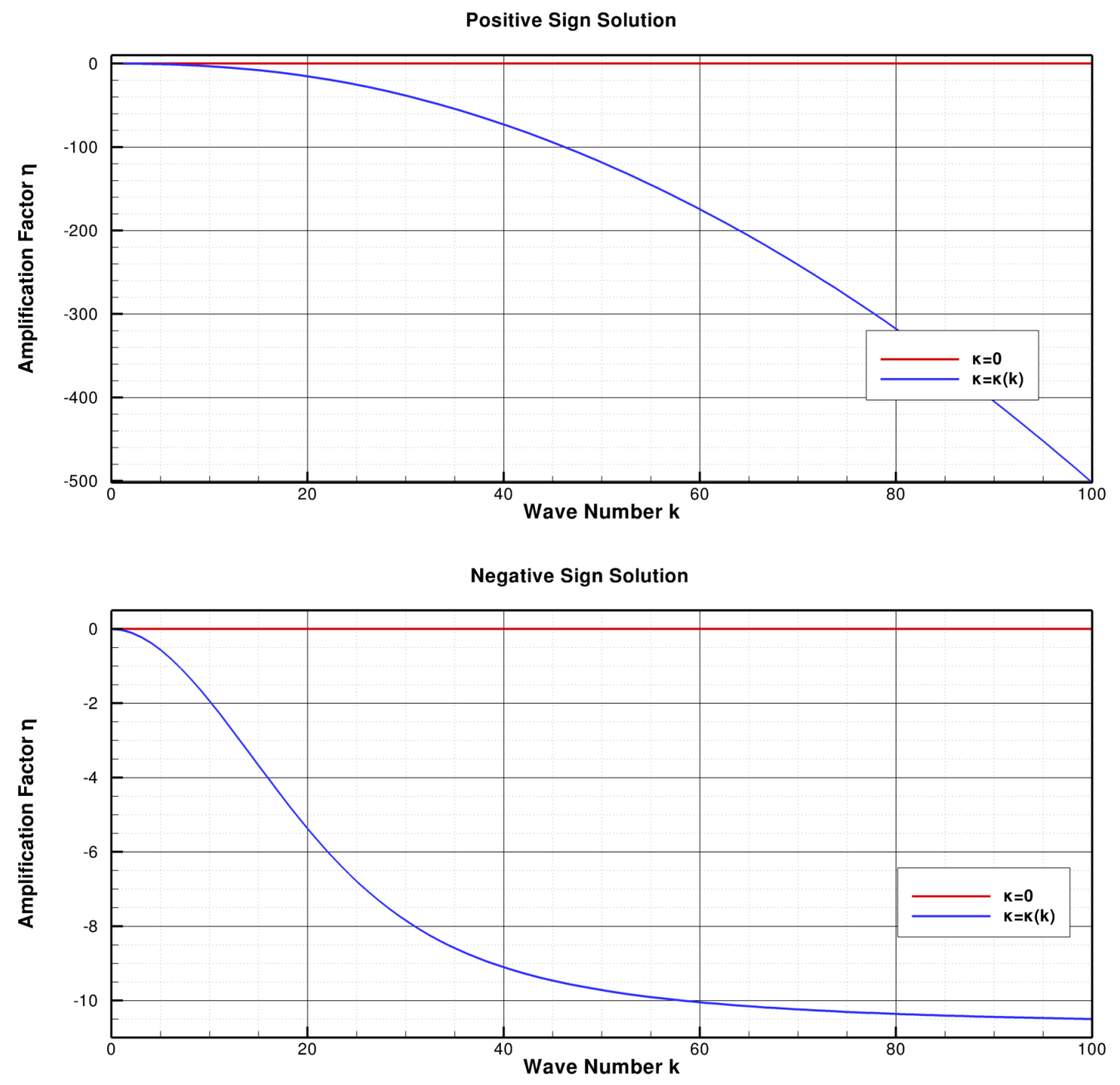

Figure 3.1: Physical consistency test of the expansional viscosity model with the following values for the physical parameters: $R e_{\kappa}=1,0, \gamma \beta=1,1, r_{M}=1,05$ e $\omega \tau=1,0$.

the derivative behavior changes and the amplification factor rises until an asymptotic value is reached as $k$ goes to infinity. The proportion of $\eta$ with the physical paramter $r_{M}$ changes in the same way as the previous case.

Figure 3.5 shows the sensivity of the physical parameter $\gamma \beta$, that is the product of the material constants of the barotropic relation 3.90 on the non-dimensional form. It can be seen that for small values of wave number $k$ there is smaller influence of this parameter for both solutions of the amplification factor $\eta$. For the negative sign solution, stronger effects of the amplification factor are observed for higher values of $\gamma \beta$. In this case an asymptotic behavior of the amplification factor is also observed for large values of $k$. 

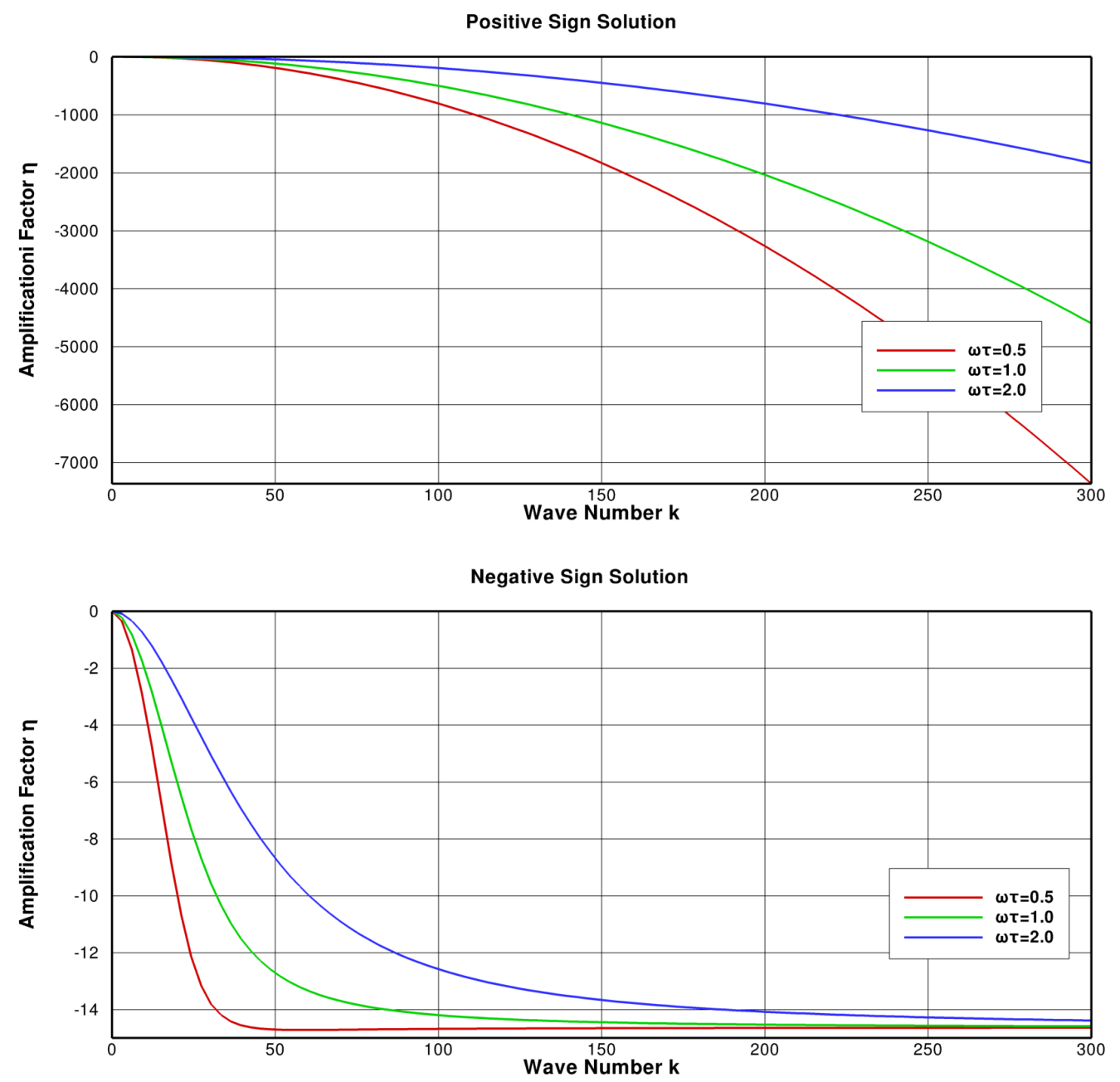

Figure 3.2: $\omega \tau$ sensitivity for the following physical parameters: $R e_{\kappa}=1,0$, $\gamma \beta=1,1, r_{M}=1,05$.

About the sensitivity analysis of the physical parameters from the amplification factor, two main trends were observed according to the solution given by equation 3.130: the behavior of both solutions for small values of wave number is basically the same, because there are no real values in the square root term of these solutions. For certain values of $k$, the behavior of these solutions changes for all the values for the physical parameters. For the positive sign solution, the growth of the amplification factor as $k$ grows is maintained, for the negative sign solution, there is a rapid growth of the amplification factor, then an asymptotic behavior as $k$ tends to infinity. Here, an inversion of the proportion with all the physical parameters can be observed in this region. Physically, the expected behavior of the expansional viscosity is the one observed with the positive sign solution, where waves 

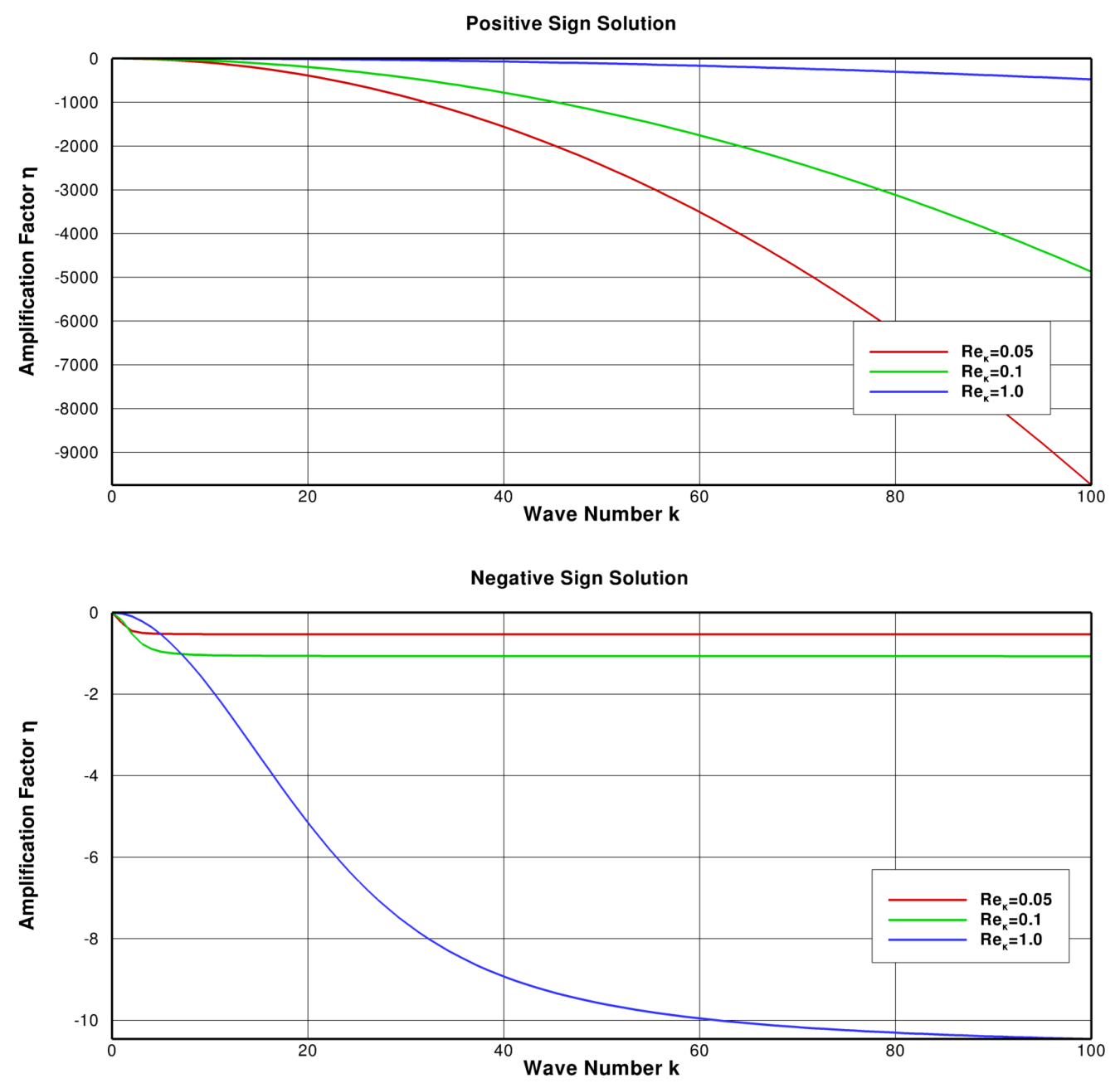

Figure 3.3: Expansional Reynolds number $R e_{\kappa}$ sensitivity for the following physical parameters: $\gamma \beta=1,1, r_{M}=1,05, \omega \tau=1,05$.

with larger wave number suffer attenuation of at least two orders of magnitude difference between the two $\eta$ solutions. On the general solution, that is the linear combination of both solutions, $\eta_{1}$ will be the prevailing term and its parameters behavior will dictate the results. For small values of $k$ both solutions have similar values of $\eta$ and the same behavior regarding the physical parameters can be observed. 

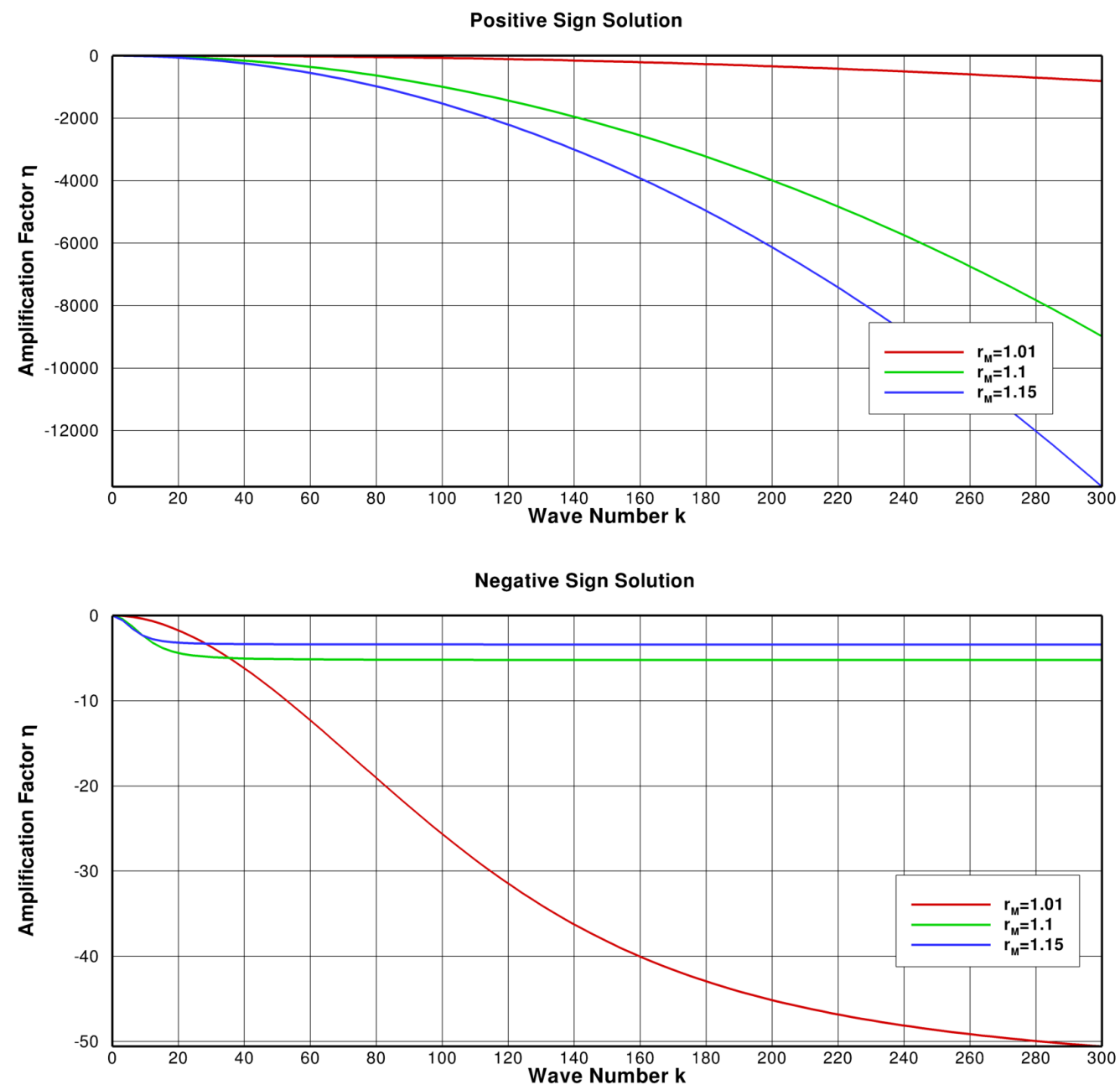

Figure 3.4: Velocity ratio $r_{M}$ sensitivity for the following physical parameters: $\gamma \beta=1,1$, $\operatorname{Re}_{\kappa}=1,0, \omega \tau=1,05$. 

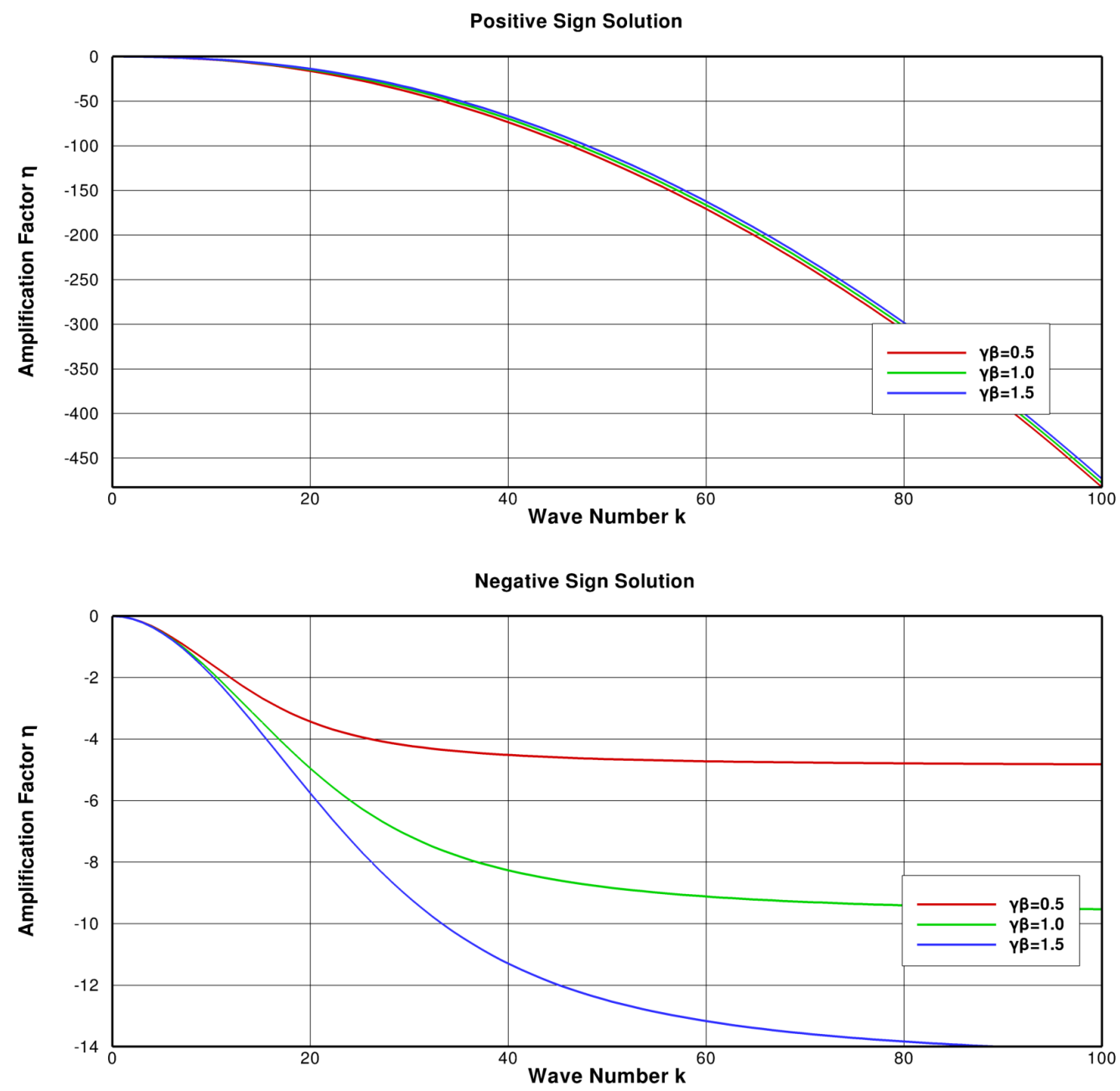

Figure 3.5: Material constants $\gamma \beta$ sensitivity for the following physical parameters: $R e_{\kappa}=1,0, r_{M}=1,05, \omega \tau=1,05$. 


\section{Chapter 4}

\section{Numerical Methodologies}

This Chapter describes the numerical methodologies of this work. A new numerical scheme for the expansional viscosity analysis is proposed and some modifications of the already existing immersed boundary method are also proposed.

The first numerical methodology that is described in this chapter is to assess in unsteady and one dimensional cases the expansional viscosity model described on chapter 3. Spatial discretization scheme of compact high order finite differences and time marching process using an explicit Runge-Kutta method are presented. Numerical stability is achieved by high spatial wave number filtering, from which typically originates the numerical oscillations. The code validation process is done by comparing a discontinuous exact solution of the Burgers equation of the numerical results to modifications on the Euler equations to make an equivalent system of governing equations, enabling a direct comparison of both solutions.

The second numerical methodology described in this chapter is the moving immersed boundary of the VAT (Virtual Aeroacoustic Tunnel) code. The spatial discretization schemes of the finite volume formulation are described along with the time marching process by using an explicit Runge-Kutta method and stabilization process with a numerical viscosity scheme. The most general case of the immersed boundary method is presented with the imposition of the boundary conditions for moving surfaces. 


\subsection{Expansional Viscosity Numerical Model}

The numerical methodology for solving the system of differential equations with the expansional viscosity is presented. A finite difference method for the space discretization is time marched with a Runge-Kutta method, both with very high spatial and temporal precision respectively. A spectral high order filter is used to eliminate the high wave number content to achieve numerical stability.

For the numerical methodology validation, a simplification of the system of equations to the viscous Burgers' equation is made. The numerical results are then compared with the analytical solution of a traveling discontinuity. The numerical precision is then evaluated for several values of the non dimensional parameter. The difference of these solutions is then evaluated for the validation of the numerical scheme and its computational implementation.

\subsubsection{Governing Equations}

For the sake of clarity, we show again the system of equations obtained with the expansional viscosity model from chapter 3 :

- Continuity equation:

$$
\frac{\partial \rho}{\partial t}+\rho \nabla \cdot \mathbf{u}+\mathbf{u} \cdot \nabla \rho=0
$$

- Linear momentum equation:

$$
\rho\left(\frac{\partial \mathbf{u}}{\partial t}+\mathbf{u} \cdot \nabla \mathbf{u}\right)+\nabla p_{0}-\nabla(\kappa \nabla \cdot \mathbf{u})=0
$$

- Expansional viscosity in frequency domain:

$$
\kappa=\frac{\rho \tau c_{0}^{2}}{1-i \omega \tau}\left(r_{M}^{2}-1\right) .
$$

- Barotropic equation:

$$
p_{0}(\rho)=\beta \rho^{\gamma}
$$

Putting the continuity and momentum equations into the one dimensional form: 
- Continuity equation:

$$
\frac{\partial \rho}{\partial t}+\rho \frac{\partial u}{\partial x}+u \frac{\partial \rho}{\partial x}=0 .
$$

- Linear momentum equation:

$$
\rho \frac{\partial u}{\partial t}+\rho u \frac{\partial u}{\partial x}+\frac{\partial p_{0}}{\partial x}-\frac{\partial}{\partial x}\left(\kappa \frac{\partial u}{\partial x}\right)=0 .
$$

- Expansional viscosity in frequency domain:

$$
\kappa=\frac{\rho \tau c_{0}^{2}}{1-i \omega \tau}\left(r_{M}^{2}-1\right) .
$$

- Barotropic equation:

$$
p_{0}(\rho)=\beta \rho^{\gamma} .
$$

In chapter 3 the expansional viscosity model is obtained in the spectral domain. To make it applicable in the time domain, it is necessary to apply the time derivative operator, as stated by equation 3.48. The imaginary oscillation frequency $i \omega$ is then swapped with the time derivative:

$$
-i \omega \kappa=-\frac{\kappa}{\tau}+\rho c_{0}^{2}\left(r_{M}^{2}-1\right),
$$

where the time domain equivalent equation is given by:

$$
\frac{\partial \kappa}{\partial t}=-\frac{\kappa}{\tau}+\rho c_{0}^{2}\left(r_{M}^{2}-1\right) .
$$

Note that the equation 4.10 is the time domain form of the expansional viscosity model, making it an evolution equation in time domain.

By applying the proposed non dimensional terms from section 3.2.1, the continuity, momentum and barotropic equations are then given by:

- Continuity equation:

$$
\frac{\partial \rho^{*}}{\partial t^{*}}+\rho^{*} \frac{\partial u^{*}}{\partial x^{*}}+u^{*} \frac{\partial \rho^{*}}{\partial x^{*}}=0
$$

- Linear momentum equation:

$$
\rho \frac{\partial u^{*}}{\partial t^{*}}+\rho^{*} u^{*} \frac{\partial u^{*}}{\partial x^{*}}+\frac{\partial p_{0}^{*}}{\partial x^{*}}-\frac{1}{R e_{\kappa}} \frac{\partial}{\partial x^{*}}\left(\kappa^{*} \frac{\partial u^{*}}{\partial x^{*}}\right)=0 .
$$


- Barotropic equation:

$$
p_{0}^{*}\left(\rho^{*}\right)=\beta^{*}\left(\rho^{*}\right)^{\gamma}
$$

for the expansional viscosity term $\kappa$ we use the same non dimensional substitutions from the linear stability analysis:

$$
\kappa^{*}=\frac{\kappa}{\rho_{0} c_{0}^{2} \tau}
$$

By applying the non dimensional substitutions into the evolution equation of the expansional viscosity, we have:

$$
\frac{\partial \kappa^{*}}{\partial t^{*}}=\frac{L_{0}}{U_{0} \tau}\left(-\kappa^{*}+\rho^{*}\left(r_{M}^{2}-1\right)\right)
$$

Now defining the relaxation time $\tau$ as a physical parameter, the term $L_{0} /\left(U_{0} \tau\right)$ is then identified as a new non dimensional parameter for the time domain. This parameter can be defined as:

$$
\Psi=\frac{L_{0}}{U_{0} \tau}
$$

This physical parameter is the ratio of the characteristic flow time and the relaxation time related to the thermodynamic equilibrium of the fluid.

The final, non dimensional and conservative form of the system of differential equations where the numerical scheme will be applied is:

- Continuity equation:

$$
\frac{\partial \rho^{*}}{\partial t^{*}}+\frac{\partial}{\partial x^{*}}\left(\rho^{*} u^{*}\right)=0
$$

- Linear momentum equation:

$$
\frac{\partial}{\partial t^{*}}\left(\rho^{*} u^{*}\right)+\frac{\partial}{\partial x^{*}}\left(\rho^{*} u^{*} u^{*}\right)+\frac{\partial p_{0}^{*}}{\partial x^{*}}-\frac{1}{R e_{\kappa}}\left(\frac{\partial \kappa^{*}}{\partial x^{*}} \frac{\partial u^{*}}{\partial x^{*}}+\kappa^{*} \frac{\partial^{2} u^{*}}{\partial x^{* 2}}\right)=0 .
$$

- Time domain expansional viscosity equation:

$$
\frac{\partial \kappa^{*}}{\partial t^{*}}=\Psi\left(-\kappa^{*}+\rho^{*}\left(r_{M}^{2}-1\right)\right)
$$

- Barotropic equation:

$$
p_{0}^{*}\left(\rho^{*}\right)=\beta^{*}\left(\rho^{*}\right)^{\gamma}
$$




\subsubsection{Numerical Method}

From the system of differential equations obtained in section 4.1.1, we start with the Padé scheme [81] for the first order space derivatives:

$$
\beta f_{i-2}^{\prime}+\alpha f_{i-1}^{\prime}+f_{i}^{\prime}+\alpha f_{i+1}^{\prime}+\beta f_{i+2}^{\prime}=c \frac{\left(f_{i+3}-f_{i-3}\right)}{6 \Delta x}+b \frac{\left(f_{i+2}-f_{i-2}\right)}{4 \Delta x}+a \frac{\left(f_{i+1}-f_{i-1}\right)}{2 \Delta x} .
$$

Equation 4.21 defines an implicit algebraic relation between the spatial derivatives $f^{\prime}$ at the computational mesh points and the values of the given unknown variable $f$. A linear system of equations must be solved to obtain the derivatives. The coefficients $\alpha, \beta, a, b$, and $c$ can be obtained according with the desired capabilities of the numerical scheme. Two main characteristics are considered here; the formal order of precision and spectral capabilities. The formal order of precision is the order of the truncating error when the Taylor series are applied on equation 4.21. The spectral capabilities are the restraints applied with the Fourier transform on equation 4.21 to obtain good discretization characteristics for a range of wavenumbers.

The coefficients of equation 4.21 can be regarded as degrees of freedom to be used with the desired characteristics described above. In the numerical scheme used here, two degrees of freedom were used for the formal fourth order of precision and the remaining three degrees of freedom were used for the optimization in the spectral domain. By applying the Taylor series on equation 4.21 and using two degrees of freedom to obtain a fourth order numerical scheme, the following restraints are obtained [81]:

$$
\begin{array}{r}
a+b+c=1+2 \alpha+2 \beta \text { on the order of }(\Delta x)^{0} \\
a+2^{2} b+3^{2} c=2 \frac{3 !}{2 !}\left(\alpha+2^{2} \beta\right) \text { on the order of }(\Delta x)^{2} .
\end{array}
$$

An infinite set of solutions exist when equations 4.22 and 4.23 are used to obtain the coefficients from equation 4.21 for a fourth order scheme. The remaining three degrees of freedom are used for a spectral optimization. By applying the Fourier transform on equation 4.21 the following spectral relation is obtained [81]:

$$
\begin{aligned}
k^{\prime}(k)(1+2 \alpha \cos (2 \pi k \Delta x) & +2 \beta \cos (2 \pi k \Delta x))= \\
& a \sin (2 \pi k \Delta x)+(b / 2) \sin (4 \pi k \Delta x)+(c / 3) \sin (6 \pi k \Delta x)
\end{aligned}
$$


where $k$ is a given numerical wave number of a discretized single wave and $k^{\prime}(k)$ is the modified wavenumber. The following restraints were applied in the spectral domain [81]:

$$
\begin{aligned}
& k^{\prime}\left(2 \pi k \Delta x_{1}\right)=2 \pi k \Delta x_{1}=2.2, \\
& k^{\prime}\left(2 \pi k \Delta x_{2}\right)=2 \pi k \Delta x_{2}=2.3, \\
& k^{\prime}\left(2 \pi k \Delta x_{3}\right)=2 \pi k \Delta x_{3}=2.4,
\end{aligned}
$$

resulting in the following relations:

$$
\begin{aligned}
& 2.2(1+2 \alpha \cos (2.2)+2 \beta \cos (2.2))=a \sin (2.2)+(b / 2) \sin (4.4)+(c / 3) \sin (6.6), \\
& 2.3(1+2 \alpha \cos (2.3)+2 \beta \cos (2.3))=a \sin (2.3)+(b / 2) \sin (4.6)+(c / 3) \sin (6.9), \\
& 2.4(1+2 \alpha \cos (2.4)+2 \beta \cos (2.4))=a \sin (2.4)+(b / 2) \sin (4.8)+(c / 3) \sin (7.2) .
\end{aligned}
$$

With the restraints set in equations $4.22,4.23$, and from 4.26 to 4.28 , the coefficients of the scheme can be obtained. The following coefficients were found in the literature for single precision numerical implementations [81]:

$$
\begin{gathered}
a=1.3025166, \quad b=0.9935500, \quad c=0.03750245, \\
\alpha=0.5771439, \quad \beta=0.0896406 .
\end{gathered}
$$

By solving the resulting linear system of equations, we have the following coefficients for the first order finite difference scheme in double precision:

$$
\begin{gathered}
a=1.294985463981978, \quad b=1.012134117804698, \quad c=0.040558683106297, \\
\alpha=0.581310505308791, \quad \beta=0.092528627137696 .
\end{gathered}
$$

Note that there is some numerical differences in the given coefficients and the calculated ones. The most probable cause was the method used to solve the linear system of equations to obtain the finite difference coefficients. For the double precision form of the coefficients, the program used to solve the linear system was Matlab R2012a for Linux 64 bits.

To calculate the derivatives, a linear system must be solved for each mesh point according with equation 4.21 . For a mesh with $N$ equally spaced points, the following linear system is obtained: 


$$
\begin{aligned}
& {\left[\begin{array}{ccccccccccccccc}
\beta & \alpha & 1 & \alpha & \beta & 0 & 0 & \ldots & 0 & 0 & 0 & 0 & 0 & 0 & 0 \\
0 & \beta & \alpha & 1 & \alpha & \beta & 0 & \ldots & 0 & 0 & 0 & 0 & 0 & 0 & 0 \\
0 & 0 & \beta & \alpha & 1 & \alpha & \beta & \ldots & 0 & 0 & 0 & 0 & 0 & 0 & 0 \\
\vdots & \vdots & \vdots & \vdots & \vdots & \vdots & \vdots & \ddots & \vdots & \vdots & \vdots & \vdots & \vdots & \vdots & \vdots \\
0 & 0 & 0 & 0 & 0 & 0 & 0 & \ldots & \beta & \alpha & 1 & \alpha & \beta & 0 & 0 \\
0 & 0 & 0 & 0 & 0 & 0 & 0 & \ldots & 0 & \beta & \alpha & 1 & \alpha & \beta & 0 \\
0 & 0 & 0 & 0 & 0 & 0 & 0 & \ldots & 0 & 0 & \beta & \alpha & 1 & \alpha & \beta
\end{array}\right]\left[\begin{array}{c}
f_{-1}^{\prime} \\
f_{0}^{\prime} \\
f_{1}^{\prime} \\
\vdots \\
f_{N}^{\prime} \\
f_{N+1}^{\prime} \\
f_{N+2}^{\prime}
\end{array}\right]} \\
& =\left[\begin{array}{c}
c \frac{\left(f_{4}-f_{-2}\right)}{6 \Delta x}+b \frac{\left(f_{3}-f_{-1}\right)}{4 \Delta x}+a \frac{\left(f_{2}-f_{0}\right)}{2 \Delta x} \\
c \frac{\left(f_{5}-f_{-1}\right)}{6 \Delta x}+b \frac{\left(f_{4}-f_{0}\right)}{4 \Delta x}+a \frac{\left(f_{3}-f_{1}\right)}{2 \Delta x} \\
c \frac{\left(f_{6}-f_{0}\right)}{6 \Delta x}+b \frac{\left(f_{5}-f_{1}\right)}{4 \Delta x}+a \frac{\left(f_{4}-f_{2}\right)}{2 \Delta x} \\
\vdots \\
c \frac{\left(f_{N+1}-f_{N-5}\right)}{6 \Delta x}+b \frac{\left(f_{N}-f_{N-4}\right)}{4 \Delta x}+a \frac{\left(f_{N-1}-f_{N-3}\right)}{2 \Delta x} \\
c \frac{\left(f_{N+2}-f_{N-4}\right)}{6 \Delta x}+b \frac{\left(f_{N+1}-f_{N-3}\right)}{4 \Delta x}+a \frac{\left(f_{N}-f_{N-2}\right)}{2 \Delta x} \\
c \frac{\left(f_{N+3}-f_{N-3}\right)}{6 \Delta x}+b \frac{\left(f_{N+2}-f_{N-2}\right)}{4 \Delta x}+a \frac{\left(f_{N+1}-f_{N-1}\right)}{2 \Delta x}
\end{array}\right]
\end{aligned}
$$

Note that the values of variables on nodes with index less than 1 and higher than $N$ must be provided according with the boundary conditions, resulting on the removal of the first two and last two lines of unknown variables of the system of equations 4.31, and thus, reducing the system to $N$ unknown variables. Another boundary condition that is used for the infinite wave is the cyclic boundary condition. Equation 4.21 will result in the following linear system: 


$$
\begin{aligned}
& {\left[\begin{array}{ccccccccccc}
1 & \alpha & \beta & 0 & 0 & \ldots & 0 & 0 & 0 & \beta & \alpha \\
\alpha & 1 & \alpha & \beta & 0 & \ldots & 0 & 0 & 0 & 0 & \beta \\
\beta & \alpha & 1 & \alpha & \beta & \ldots & 0 & 0 & 0 & 0 & 0 \\
\vdots & \vdots & \vdots & \vdots & \vdots & \ddots & \vdots & \vdots & \vdots & \vdots & \vdots \\
0 & 0 & 0 & 0 & 0 & \ldots & \beta & \alpha & 1 & \alpha & \beta \\
\beta & 0 & 0 & 0 & 0 & \ldots & 0 & \beta & \alpha & 1 & \alpha \\
\alpha & \beta & 0 & 0 & 0 & \ldots & 0 & 0 & \beta & \alpha & 1
\end{array}\right]\left[\begin{array}{c}
f_{1}^{\prime} \\
f_{2}^{\prime} \\
f_{3}^{\prime} \\
\vdots \\
f_{N-2}^{\prime} \\
f_{N-1}^{\prime} \\
f_{N}^{\prime}
\end{array}\right]} \\
& =\left[\begin{array}{c}
c \frac{\left(f_{4}-f_{N-3}\right)}{6 \Delta x}+b \frac{\left(f_{3}-f_{N-2}\right)}{4 \Delta x}+a \frac{\left(f_{2}-f_{N-1}\right)}{2 \Delta x} \\
c \frac{\left(f_{5}-f_{N-4}\right)}{6 \Delta x}+b \frac{\left(f_{4}-f_{N-3}\right)}{4 \Delta x}+a \frac{\left(f_{3}-f_{N-2}\right)}{2 \Delta x} \\
c \frac{\left(f_{6}-f_{N-5}\right)}{6 \Delta x}+b \frac{\left(f_{5}-f_{N-4}\right)}{4 \Delta x}+a \frac{\left(f_{4}-f_{N-3}\right)}{2 \Delta x} \\
\vdots \\
c \frac{\left(f_{1}-f_{N-5}\right)}{6 \Delta x}+b \frac{\left(f_{N}-f_{N-4}\right)}{4 \Delta x}+a \frac{\left(f_{N-1}-f_{N-3}\right)}{2 \Delta x} \\
c \frac{\left(f_{2}-f_{N-4}\right)}{6 \Delta x}+b \frac{\left(f_{1}-f_{N-3}\right)}{4 \Delta x}+a \frac{\left(f_{N}-f_{N-2}\right)}{2 \Delta x} \\
c \frac{\left(f_{3}-f_{N-3}\right)}{6 \Delta x}+b \frac{\left(f_{2}-f_{N-2}\right)}{4 \Delta x}+a \frac{\left(f_{1}-f_{N-1}\right)}{2 \Delta x}
\end{array}\right]
\end{aligned}
$$

A similar numerical scheme can be obtained for the space discretization of the second order derivative. We use the general compact formulation:

$$
\begin{aligned}
& \beta f_{i-2}^{\prime \prime}+\alpha \beta f_{i-1}^{\prime \prime}+f_{i}^{\prime \prime}+\alpha f_{i+1}^{\prime \prime}+\beta f_{i+2}^{\prime \prime}= \\
& c \frac{\left(f_{i+3}-2 f_{i}+f_{i-3}\right)}{9 \Delta x^{2}}+b \frac{\left(f_{i+2}-2 f_{i}+f_{i-2}\right)}{4 \Delta x^{2}}+a \frac{\left(f_{i+1}-2 f_{i}+f_{i-1}\right)}{\Delta x^{2}}
\end{aligned}
$$

The main idea behind the calculation of the coefficients for the second order spatial derivative is the same for the first order case: two degrees of freedom for the formal precision of the scheme and the remaining three for the spectral optimization, where the formal fourth order of numerical precision is also kept. By applying the Taylor series in equation 4.33 to obtain the restraints up to the fourth order of formal precision [81]:

$$
\begin{aligned}
a+b+c & =1+2 \alpha+2 \beta \text { on the order of }(\Delta x)^{0}, \\
a+2^{2} b+3^{2} c & =\frac{4 !}{2 !}\left(\alpha+2^{2} \beta\right) \text { on the order of }(\Delta x)^{2} .
\end{aligned}
$$

On the same way that was done with the first order equation 4.21, the Fourier transform 
is applied on equation 4.33 and the following spectral relation is obtained [81]:

$$
\begin{aligned}
k^{\prime \prime}(k)(1+2 \alpha \cos (k)+2 \beta \cos (2 k)) & \\
& =2 a(1-\cos (k))+(b / 2)(1-\cos (2 k))+(2 c / 9)(1-\cos (3 k)) .
\end{aligned}
$$

With the following restraints of the spectral domain:

$$
\begin{aligned}
& k^{\prime \prime}\left(2 \pi k \Delta x_{1}\right)=2 \pi k \Delta x_{1}=2.0^{2}, \\
& k^{\prime \prime}\left(2 \pi k \Delta x_{2}\right)=2 \pi k \Delta x_{2}=2.4^{2}, \\
& k^{\prime \prime}\left(2 \pi k \Delta x_{3}\right)=2 \pi k \Delta x_{3}=2.6^{2},
\end{aligned}
$$

the following relations are obtained:

$$
\begin{aligned}
& 2.0^{2}\left(1+2 \alpha \cos \left(2.0^{2}\right)+2 \beta \cos \left(2 * 2.0^{2}\right)\right) \\
& \quad=2 a\left(1-\cos \left(2.0^{2}\right)\right)+(b / 2)\left(1-\cos \left(2 * 2.0^{2}\right)\right)+(2 c / 9)\left(1-\cos \left(3 * 2.0^{2}\right)\right), \\
& 2.4^{2}\left(1+2 \alpha \cos \left(2.4^{2}\right)+2 \beta \cos \left(2 * 2.4^{2}\right)\right) \\
& \quad=2 a\left(1-\cos \left(2.4^{2}\right)\right)+(b / 2)\left(1-\cos \left(2 * 2.4^{2}\right)\right)+(2 c / 9)\left(1-\cos \left(3 * 2.4^{2}\right)\right), \\
& 2.6^{2}\left(1+2 \alpha \cos \left(2.6^{2}\right)+2 \beta \cos \left(2 * 2.6^{2}\right)\right) \\
& \quad=2 a\left(1-\cos \left(2.6^{2}\right)\right)+(b / 2)\left(1-\cos \left(2 * 2.6^{2}\right)\right)+(2 c / 9)\left(1-\cos \left(3 * 2.6^{2}\right)\right) .
\end{aligned}
$$

With two restraints for the formal order of precision and three in the spectral domain, a resulting linear system of equations is obtained with equations $4.34,4.35$, and from 4.38 to 4.40. The following coefficients for the second order in single precision are given by [81]:

$$
\begin{gathered}
a=0.21564935, \quad b=1.7233220, \quad c=0.17659730 \\
\alpha=0.50209266, \quad \beta=0.05569169,
\end{gathered}
$$

the values for double precision calculated here are:

$$
\begin{gathered}
a=0.215649631004306, \quad b=1.723321721695239, \quad c=0.176597105958252 \\
\alpha=0.502092570454933, \quad \beta=0.055691658873965 .
\end{gathered}
$$


In a similar way, a linear system of equations must be solved to obtain the second order derivatives:

$$
\begin{aligned}
& {\left[\begin{array}{ccccccccccccccc}
\beta & \alpha & 1 & \alpha & \beta & 0 & 0 & \ldots & 0 & 0 & 0 & 0 & 0 & 0 & 0 \\
0 & \beta & \alpha & 1 & \alpha & \beta & 0 & \ldots & 0 & 0 & 0 & 0 & 0 & 0 & 0 \\
0 & 0 & \beta & \alpha & 1 & \alpha & \beta & \ldots & 0 & 0 & 0 & 0 & 0 & 0 & 0 \\
\vdots & \vdots & \vdots & \vdots & \vdots & \vdots & \vdots & \ddots & \vdots & \vdots & \vdots & \vdots & \vdots & \vdots & \vdots \\
0 & 0 & 0 & 0 & 0 & 0 & 0 & \ldots & \beta & \alpha & 1 & \alpha & \beta & 0 & 0 \\
0 & 0 & 0 & 0 & 0 & 0 & 0 & \ldots & 0 & \beta & \alpha & 1 & \alpha & \beta & 0 \\
0 & 0 & 0 & 0 & 0 & 0 & 0 & \ldots & 0 & 0 & \beta & \alpha & 1 & \alpha & \beta
\end{array}\right]\left[\begin{array}{c}
f_{-1}^{\prime \prime} \\
f_{0}^{\prime \prime} \\
f_{1}^{\prime \prime} \\
\vdots \\
f_{N}^{\prime \prime} \\
f_{N+1}^{\prime \prime} \\
f_{N+2}^{\prime \prime}
\end{array}\right]} \\
& =\left[\begin{array}{c}
c \frac{\left(f_{4}-2 f_{i}+f_{-2}\right)}{6 \Delta x}+b \frac{\left(f_{3}-2 f_{i}+f_{-1}\right)}{4 \Delta x}+a \frac{\left(f_{2}-2 f_{i}+f_{0}\right)}{2 \Delta x} \\
c \frac{\left(f_{5}-2 f_{i}+f_{-1}\right)}{6 \Delta x}+b \frac{\left(f_{4}-2 f_{i}+f_{0}\right)}{4 \Delta x}+a \frac{\left(f_{3}-2 f_{i}+f_{1}\right)}{2 \Delta x} \\
c \frac{\left(f_{6}-2 f_{i}+f_{0}\right)}{6 \Delta x}+b \frac{\left(f_{5}-2 f_{i}+f_{1}\right)}{4 \Delta x}+a \frac{\left(f_{4}-2 f_{i}+f_{2}\right)}{2 \Delta x} \\
\vdots \\
c \frac{\left(f_{N+1}-2 f_{i}+f_{N-5}\right)}{6 \Delta x}+b \frac{\left(f_{N}-2 f_{i}+f_{N-4}\right)}{4 \Delta x}+a \frac{\left(f_{N-1}-2 f_{i}+f_{N-3}\right)}{2 \Delta x} \\
c \frac{\left(f_{N+2}-2 f_{i}+f_{N-4}\right)}{6 \Delta x}+b \frac{\left(f_{N+1}-2 f_{i}+f_{N-3}\right)}{4 \Delta x}+a \frac{\left(f_{N}-2 f_{i}+f_{N-2}\right)}{2 \Delta x} \\
c \frac{\left(f_{N+3}-2 f_{i}+f_{N-3}\right)}{6 \Delta x}+b \frac{\left(f_{N+2}-2 f_{i}+f_{N-2}\right)}{4 \Delta x}+a \frac{\left(f_{N+1}-2 f_{i}+f_{N-1}\right)}{2 \Delta x}
\end{array}\right] .
\end{aligned}
$$

The first two lines and the last two lines of the linear system must be replaced by the boundary conditions with the derivatives and solution variables known a priori. The cyclic boundary condition results in the following linear system of second order derivatives as unknown variables: 


$$
\begin{aligned}
& {\left[\begin{array}{ccccccccccc}
1 & \alpha & \beta & 0 & 0 & \ldots & 0 & 0 & 0 & \beta & \alpha \\
\alpha & 1 & \alpha & \beta & 0 & \ldots & 0 & 0 & 0 & 0 & \beta \\
\beta & \alpha & 1 & \alpha & \beta & \ldots & 0 & 0 & 0 & 0 & 0 \\
\vdots & \vdots & \vdots & \vdots & \vdots & \ddots & \vdots & \vdots & \vdots & \vdots & \vdots \\
0 & 0 & 0 & 0 & 0 & \ldots & \beta & \alpha & 1 & \alpha & \beta \\
\beta & 0 & 0 & 0 & 0 & \ldots & 0 & \beta & \alpha & 1 & \alpha \\
\alpha & \beta & 0 & 0 & 0 & \ldots & 0 & 0 & \beta & \alpha & 1
\end{array}\right]\left[\begin{array}{c}
f_{1}^{\prime \prime} \\
f_{2}^{\prime \prime} \\
f_{3}^{\prime \prime} \\
\vdots \\
f_{N-2}^{\prime \prime} \\
f_{N-1}^{\prime \prime} \\
f_{N}^{\prime \prime}
\end{array}\right]} \\
& =\left[\begin{array}{c}
c \frac{\left(f_{4}-2 f_{i}+f_{N-3}\right)}{6 \Delta x}+b \frac{\left(f_{3}-2 f_{i}+f_{N-2}\right)}{4 \Delta x}+a \frac{\left(f_{2}-2 f_{i}+f_{N-1}\right)}{2 \Delta x} \\
c \frac{\left(f_{5}-2 f_{i}+f_{N-4}\right)}{6 \Delta x}+b \frac{\left(f_{4}-2 f_{i}+f_{N-3}\right)}{4 \Delta x}+a \frac{\left(f_{3}-2 f_{i}+f_{N-2}\right)}{2 \Delta x} \\
c \frac{\left(f_{6}-2 f_{i}+f_{N-5}\right)}{6 \Delta x}+b \frac{\left(f_{5}-2 f_{i}+f_{N-4}\right)}{4 \Delta x}+a \frac{\left(f_{4}-2 f_{i}+f_{N-3}\right)}{2 \Delta x} \\
\vdots \\
c \frac{\left(f_{1}-2 f_{i}+f_{N-5}\right)}{6 \Delta x}+b \frac{\left(f_{N}-2 f_{i}+f_{N-4}\right)}{4 \Delta x}+a \frac{\left(f_{N-1}-2 f_{i}+f_{N-3}\right)}{2 \Delta x} \\
c \frac{\left(f_{2}-2 f_{i}+f_{N-4}\right)}{6 \Delta x}+b \frac{\left(f_{1}-2 f_{i}+f_{N-3}\right)}{4 \Delta x}+a \frac{\left(f_{N}-2 f_{i}+f_{N-2}\right)}{2 \Delta x} \\
c \frac{\left(f_{3}-2 f_{i}+f_{N-3}\right)}{6 \Delta x}+b \frac{\left(f_{2}-2 f_{i}+f_{N-2}\right)}{4 \Delta x}+a \frac{\left(f_{1}-2 f_{i}+f_{N-1}\right)}{2 \Delta x}
\end{array}\right] .
\end{aligned}
$$

For the time marching process the Runge-Kutta explicit method with nine steps and of fifth order of precision [82] was used. The name of the scheme is RK5(4)9(2R+)M. According to the Runge-Kutta formulation for an initial value problem, we have:

$$
\frac{d U_{i}}{d t}=F(t, U(t))
$$

where for the governing equations 4.17 to 4.20 , we have the calculation of the time derivative to be solved as an initial value problem and the function $F$ is solved by the other space discretization schemes described before, for the first and second order derivatives.

By following the idea of the Runge-Kutta method, from the initial step $U^{(n)}$ for the numerical time step of $U^{(n+1)}$, with a physical time step of $\Delta t$, we have:

$$
\begin{array}{r}
F^{(i)}=F^{(i)}\left(t^{(i)}, U^{(i)}\right), \\
U^{(i)}=U^{(n)}+\Delta t \sum_{j=1}^{i-1} a_{i j} F^{(j)}, \\
U^{(n+1)}=U^{(n)}+\Delta t \sum_{j=1}^{9} b_{j} F^{(j)},
\end{array}
$$


where the coefficients of the scheme RK5(4)9(2R+)M are given by table 4.1.

\begin{tabular}{lc|lc}
\hline \hline$a_{21}$ & $+\frac{5573095071601}{11304125995793}$ & $b_{1}$ & $\frac{549666665015}{5899839355879}$ \\
\hline$a_{32}$ & $+\frac{315581365608}{4729744040249}$ & $b_{2}$ & $-\frac{548816778320}{9402908589133}$ \\
\hline$a_{43}$ & $+\frac{8734064225157}{30508564569118}$ & $b_{3}$ & $\frac{1672704946363}{13015471661974}$ \\
\hline$a_{54}$ & $+\frac{6457785058448}{14982850401353}$ & $b_{4}$ & $\frac{1025420337373}{5970204766762}$ \\
\hline$a_{65}$ & $+\frac{5771559441664}{18187997215013}$ & $b_{5}$ & $\frac{1524419752016}{6755273790179}$ \\
\hline$a_{76}+\frac{1906712129266}{6681214991155}$ & $b_{6}$ & $-\frac{10259399787359}{43440802207630}$ \\
\hline$a_{87}+\frac{311585568784}{2369973437185}$ & $b_{7}$ & $\frac{4242280279850}{10722460893763}$ \\
\hline$a_{98}$ & $-\frac{4840285693886}{7758383361725}$ & $b_{8}$ & $\frac{1887552771913}{6099058196803}$ \\
\hline \hline
\end{tabular}

Table 4.1: Runge-Kutta coefficients for scheme RK5(4)9(2R+)M.

The numerical stabilization is achieved by a high spectral order explicit filter [25]. The filter removes the high frequency waves of the solution that could result in an unstable code, due to the known high wavenumber numerically unstable content. The filter is applied on the conservative variables of the Runge-Kutta steps after a certain number of iterations were completed. The filter used in this scheme is a 7-point stencil filter. It is symmetric to avoid amplification in all the wave number spectra. The most general form of a seven point stencil filter is given by:

$$
\hat{f}_{i}=A\left(f_{i+3}+f_{i-3}\right)+B\left(f_{i+2}+f_{i-2}\right)+C\left(f_{i+1}+f_{i-1}\right)+D f_{i},
$$

where the filter is applied over the solution variable $f$ and $\hat{f}$ is the resulting filtered variable.

To obtain the filter coefficients $A, B, C$ and $D$, we apply the inverse Fourier transform into equation 4.47 [25]:

$$
F(k)=2 A \cos (3 k)+2 B \cos (2 k)+2 C \cos (k)+D,
$$

where $F(k)$ is the transfer function of the filtered values over the wave number spectrum $(0, \pi)$. The transfer function represents the fraction of the original value that is conserved when the filter is applied on the variable $f_{i}$ at the mesh node $i$. For a numerically well resolved wavenumber spectrum, for example around $k=(0,1)$, the filter almost does not change the original values of the variable $f$, but for the remainder of the spectrum $(k=$ 
$(1, \pi))$, the filtered content is a fraction of the original content, as can be seen in figure 4.1 for the filter that was used in this numerical scheme.

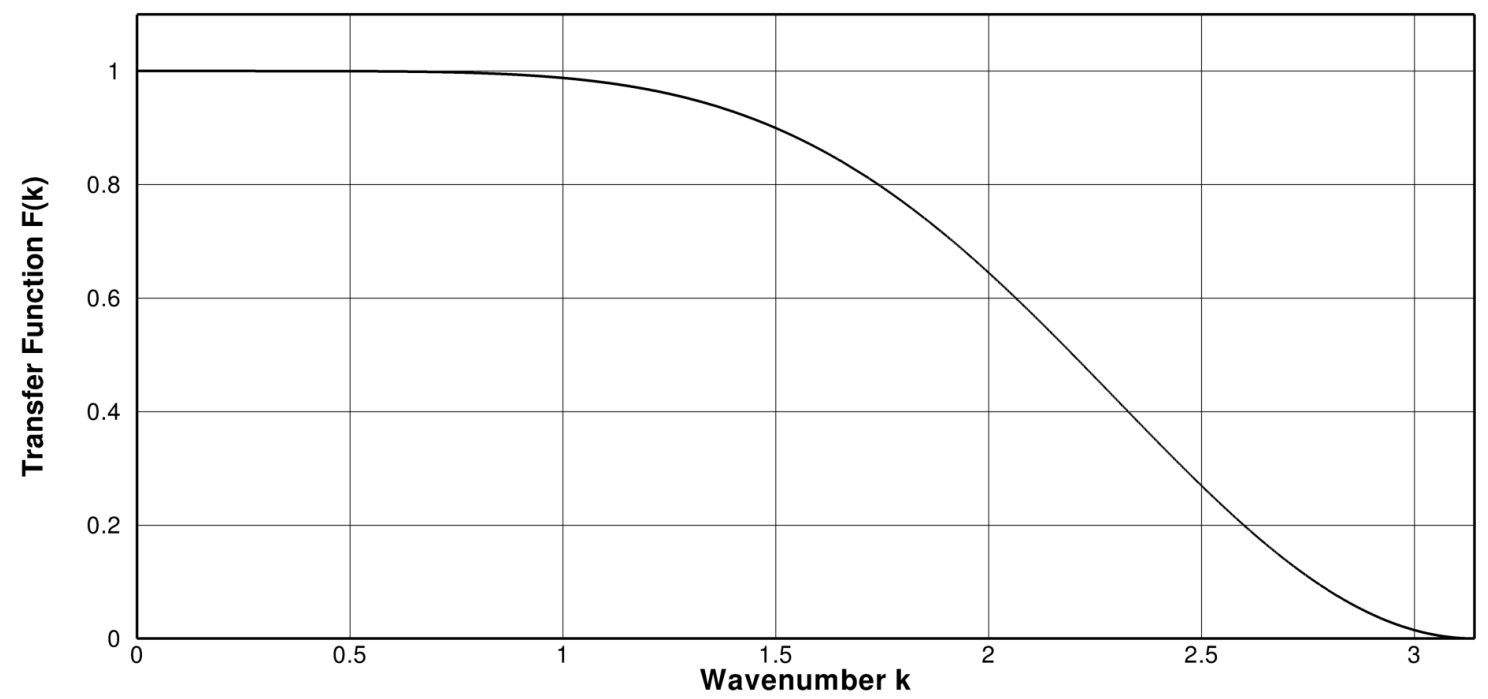

Figure 4.1: Transfer function $F(k)$ used for filtering of the poorly resolved wavenumber content.

In the 7-point stencil filter 4.47, four restraints can be used to define the spectral behavior of the filter. The following constraints are applied on equation 4.48 to obtain the low pass filter [25] shown in figure 4.1:

$$
\begin{gathered}
F(0)=2 A+2 B+2 C+D=1, \\
\frac{\partial^{2} F(0)}{\partial k^{2}}=18 A+8 B+2 C=0, \\
\frac{\partial^{4} F(0)}{\partial k^{4}}=162 A+32 B+2 C=0, \\
F(\pi)=-2 A+2 B-2 C+D=0 .
\end{gathered}
$$

By solving the resulting linear system, we obtain the following rational coefficients:

$$
A=\frac{1}{64}, \quad B=-\frac{3}{32}, \quad C=\frac{15}{64}, \quad D=\frac{11}{16} .
$$

The filter equation is then given by:

$$
\hat{f}_{i}=\frac{1}{64}\left(f_{i+3}+f_{i-3}\right)-\frac{3}{32}\left(f_{i+2}+f_{i-2}\right)+\frac{15}{64}\left(f_{i+1}+f_{i-1}\right)+\frac{11}{16} f_{i} .
$$




\subsubsection{Numerical Method Validation}

A validation process is needed to evaluate the capabilities of the proposed numerical scheme. Unfortunately the system of differential governing equations from section 4.1.1 does not have an analytic solution to be compared with the numerical solution. A physically and mathematically similar equation that has an analytical solution can be used to validate the proposed numerical scheme. The Burgers' equation is the best choice:

$$
\frac{\partial u}{\partial t}+u \frac{\partial u}{\partial x}=\nu \frac{\partial^{2} u}{\partial x^{2}}
$$

The above equation has two main characteristics that are also present in the system of governing equations from section 4.1.1: the unsteady term, the non linear convection term, and the linear second order derivative diffusion term. Physically the Burgers' equation describes the time evolution of a nonlinear wave that is dependent of the non dimensional parameter. Evanescent wave or discontinuity solutions may occur.

The Burger's equation may be defined as a special case of the system of governing equations from section 4.1.1. Considering that the fluid density is constant and has unity value, in the momentum equation the density related derivatives will be equal to zero. We then have:

$$
\frac{\partial u^{*}}{\partial t^{*}}+u^{*} \frac{\partial u^{*}}{\partial x^{*}}-\frac{1}{R e_{\kappa}}\left(\frac{\partial \kappa^{*}}{\partial x^{*}} \frac{\partial u^{*}}{\partial x^{*}}+\kappa^{*} \frac{\partial^{2} u^{*}}{\partial x^{* 2}}\right)=0 .
$$

Now we also consider that the expansional viscosity coefficient has constant value, thus eliminating the $\kappa$ related derivative in equation 4.56 :

$$
\frac{\partial u^{*}}{\partial t^{*}}+u^{*} \frac{\partial u^{*}}{\partial x^{*}}-\frac{\kappa^{*}}{R e_{\kappa}}\left(\frac{\partial^{2} u^{*}}{\partial x^{* 2}}\right)=0
$$

Now we define a numeric Reynolds number for the numerical evaluation of the obtained equation:

$$
R e=\frac{R e_{\kappa}}{\kappa^{*}} .
$$

As a result, the Burgers' equation in the non dimensional form and with the viscous terms is obtained:

$$
\frac{\partial u^{*}}{\partial t^{*}}+u^{*} \frac{\partial u^{*}}{\partial x^{*}}=\frac{1}{R e} \frac{\partial^{2} u^{*}}{\partial x^{* 2}}
$$

or in the conservative form for better numerical results [23]:

$$
\frac{\partial u^{*}}{\partial t^{*}}=-\frac{1}{2} \frac{\partial u^{* 2}}{\partial x^{*}}+\frac{1}{R e} \frac{\partial^{2} u^{*}}{\partial x^{* 2}}
$$


remembering that the usual interpretation of the parameter $1 / R e$ is the same for the NavierStokes equations; a scale relation between the convection and diffusive terms.

The analytical solution to be compared with the numerical solution is the traveling wave with discontinuity. The error from the numerical solution will assess two main issues: the dispersion (wave phase changes) and the diffusion (wave amplitude changes). The traveling wave with discontinuity analytical solution with the Burgers' equation is [83]:

$$
u(x, t)=\frac{1}{1+e^{\left(\frac{R e(x-t / 2)}{2}\right)}} .
$$

For the numerical implementation, the initial condition of the wave is the above solution with $t=0$. The boundary conditions at the ghost nodes outside the mesh are the analytical solution known values of $u, \frac{\partial u}{\partial x}$ and $\frac{\partial^{2} u}{\partial x^{2}}$. The analytical and numerical solutions will be compared after a certain physical time. The difference error is the modulus of the largest difference between the numerical and analytical solutions in the discontinuity region.

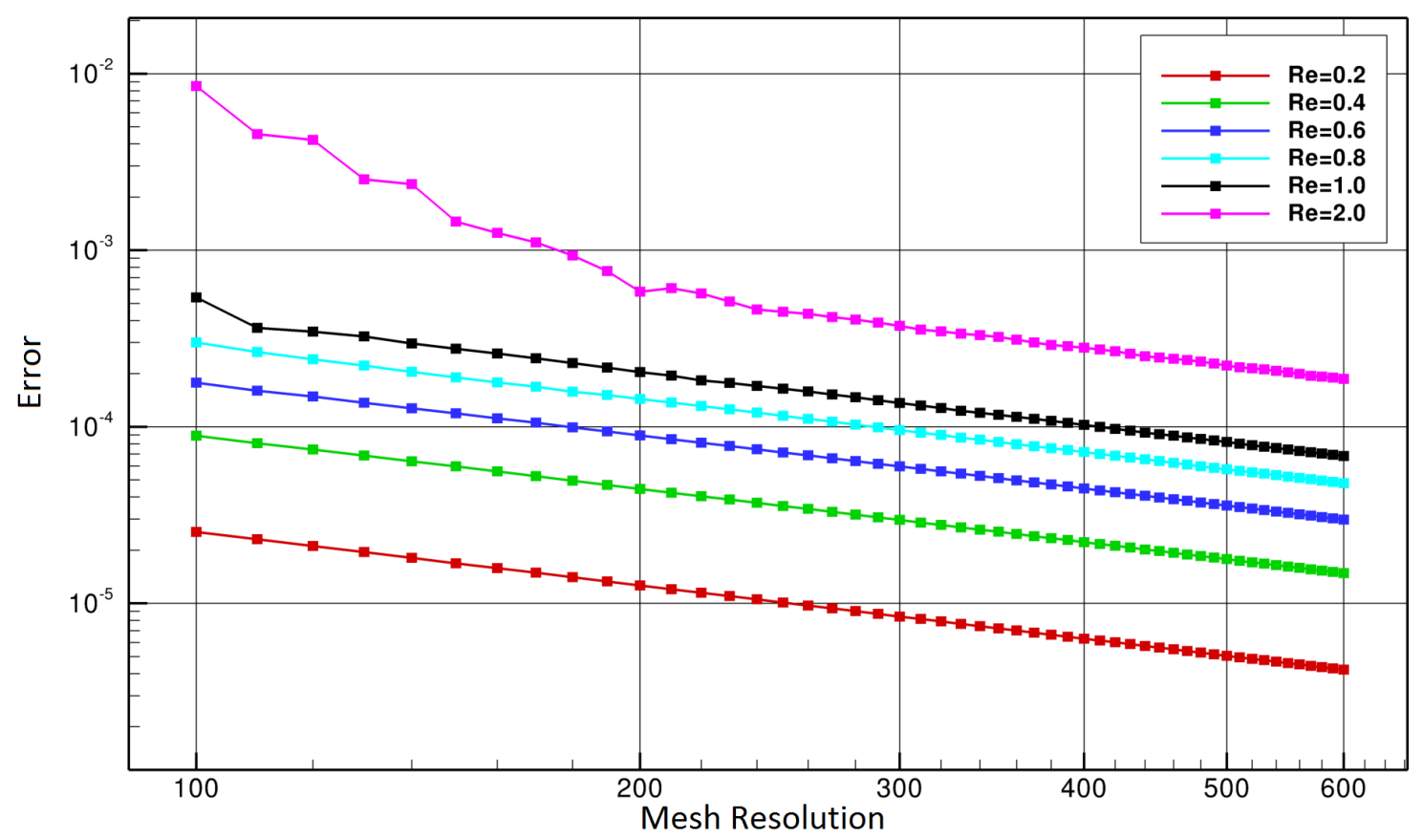

Figure 4.2: Mesh convergence of the proposed numerical scheme for the traveling wave with discontinuity solution of the Burgers' equation for several Reynolds numbers.

The value for the CFL condition used in this error assessment was 0.8 and the physical simulation time was $t^{*}=10$. The computational domain was 220 characteristic lengths. 
For this validation case, the boundary conditions on both ends of the computational mesh is the derivative equal zero for the first and second order. The imposition of the boundary conditions is automatically satisfied when the derivatives are calculated from the linear system of equation 4.21 .

Note that in figure 4.2 a well defined convergence of the numerical mesh for the studied Reynolds numbers can be observed. With higher Reynolds numbers in the traveling wave solution the solution jump tends to a discontinuity. As a consequence, less mesh points are used to describe this region with steep gradients, thus explaining the higher numerical error for higher Reynolds numbers.

\subsubsection{Implementation Details of the One Dimensional Expansional Viscosity Numerical Code}

Being a one dimensional numerical scheme, the computational cost is not as high as the other numerical scheme used in this work (VAT code). A simple personal computer was enough for the simulations.

The computational code was implemented in Fortran serial computation. For the solving of the linear systems related to equations 4.21 and 4.33 the open source numerical libraries LAPACK [84] and BLAS [85] along with the PGI compiler were used. The operating system was Linux openSUSE 12.1 x64.

\subsection{The Virtual Aeroacoustic Tunnel Numerical Methodology}

The term immersed boundary method was first used in reference to a method developed to simulate cardiac mechanics and associated blood flow [86]. The distinguishing feature of this method was that the entire simulation was carried out on a Cartesian grid, which did not conform to the geometry of the heart, and a novel procedure was formulated for imposing the effect of the immersed boundary on the flow. Since the method was introduced, numerous modifications and refinements have been proposed and a number of variants of 
this approach now exist. Initially, the immersed boundary methods were developed for incompressible viscous flows.

Immersed boundary methods for compressible viscous flows have been developed in the last years $[87,88,89,90,91,92]$. Most of these codes the resulting numerical schemes are of second-order accurate in space and time. Higher order schemes were developed to be applied on highly compressible flows [93], aerodinamically generated noise in both nonlinear [94] and linearized governing equations [95], and featured with more sophisticated discretization schemes [96, 97]. The aplication of the immersed boundary method is also suitable for high Reynolds numbers where turbulence plays a major role in certain flows. Turbulence models have been successfully applied along with the immersed boundary method: sub-grid scale models [98, 99] and full scale turbulence modelling based on the RANS equations [100]. To overcome the relative lack of resolution in the vicinity of the wall region, wall modelling for turbulence has been applied on the immersed boundary method [101, 102].

Only recently the immersed boundary method has been used in realistic simulations; oscillating cascade in turbomachinery [103], fast pitching airfoils [104], motion of a Hydro Turbine[105], real vehicle simulations [106], solid rocket motors [107].

The first version of the immersed boundary method used in this work was validated for aerodynamic cases; viscous laminar flow around a flat plate, supersonic laminar flow around a double wedge airfoil and a supersonic turbulent flow around a cylinder [108].

This metodology was validated [109] with the modal aeroacoustic prediction of a stator cascade under a rotor like linearized gust [71], generating the Tyler and Sofrin interaction modes [69] and comparing its results with the linear subsonic theory from chapter 2.

The moving version of the moving immersed boundary [111] method imposes the rotor blades movement through a regular cartesian mesh that also includes static surfaces, i.e., the stator vanes cascade. This methodology is an alternative to the classic method of sliding meshes, where there are two different computational meshes. There is one for the rotor cascade and the other static for the remaining physical domain, with a relative movement between these two meshes. A lack of resolution is always present in the interface of meshes. By using the moving immersed boundary method, low resolution regions are not in the domain, the rotor and stator cascades are both immersed in the same computational mesh. 


\subsubsection{Governing Equations}

In the proposed numerical method, the nonlinear and unsteady Euler equations will be used. It is assumed that the turbulence effects do not have a major influence on the Tyler and Sofrin mode generation process. The region that is most influenced from turbulence is usually the near wall region (boundary layer) and the wake from the rotor blades and stator vanes. For flows with high Reynolds numbers this region represents only a fraction of the interaction region between the rotor and stator, and this simplification is assumed to not have too much impact on the validation process.

For a non-inertial frame of reference, can be written in a conservation-law form as:

$$
\begin{gathered}
\frac{\partial \rho}{\partial t}+\frac{\partial}{\partial x_{i}}\left(\rho u_{i}\right)=0 \\
\frac{\partial}{\partial t}\left(\rho u_{i}\right)+\frac{\partial}{\partial x_{j}}\left(\rho u_{i} u_{j}\right)=-\frac{\partial p}{\partial x_{i}}+f_{i} \\
\frac{\partial}{\partial t}\left(\rho e_{t}\right)+\frac{\partial}{\partial x_{i}}\left(\rho e_{t} u_{i}\right)=-\frac{\partial}{\partial x_{i}}\left(p u_{i}\right)+f_{i} u_{i},
\end{gathered}
$$

where the body forces are used for the domain accelaration from an initial steady state to the reference inflow velocity. The non dimensional variables used in the above equations are defined as:

$$
\begin{gathered}
x^{*}=\frac{x}{L}, \quad y^{*}=\frac{y}{L}, \quad z^{*}=\frac{z}{L}, \quad t^{*}=\frac{t}{L / U_{\mathrm{ref}}}, \quad u^{*}=\frac{u}{U_{\mathrm{ref}}}, \quad v^{*}=\frac{v}{U_{\mathrm{ref}}}, \quad w^{*}=\frac{w}{U_{\mathrm{ref}}}, \\
p^{*}=\frac{p}{\rho_{\mathrm{ref}}\left(U_{\mathrm{ref}}\right)^{2}}, \quad \rho=\frac{\rho}{\rho_{\mathrm{ref}}}, \quad T^{*}=\frac{T}{T_{\mathrm{ref}}}, \quad e=\frac{e}{\left(U_{\mathrm{ref}}\right)^{2}}, \quad f^{*}=\frac{f}{\rho_{\mathrm{ref}}\left(U_{\mathrm{ref}}\right)^{2} / L} .
\end{gathered}
$$

The superscript ${ }^{*}$ represents non dimensional quantities and the subscript ref represents reference values. The fluid total energy is given by the sum of the internal and kinetic energies

$$
e_{t}=e+e_{k}=c_{v} T+\frac{u_{i} u_{i}}{2}
$$

and the Mach number is given by:

$$
M_{\mathrm{ref}}=\frac{U_{\mathrm{ref}}}{c_{\mathrm{ref}}}=\frac{U_{\mathrm{ref}}}{\sqrt{\gamma \mathcal{R} T_{\mathrm{ref}}}} .
$$

For a thermally and calorically perfect gas, the equation of state can be written as:

$$
p=(\gamma-1) \rho e
$$


and

$$
T=\frac{\gamma M_{\mathrm{ref}}^{2} p}{\rho}
$$

A pseudo body force $\left(f_{i}\right)$ and its pseudo body force work $\left(f_{i} u_{i}\right)$ are then introduced on the right hand side of the momentum and energy equations to continuously accelerate the flow by using a non-inertial reference frame, from the stagnation condition to the geometry reference condition, during a given acceleration time $t_{a}$. After this acceleration time, the value of the pseudo-force and pseudo-work are equal to zero, i.e., $f_{i}=f_{i} u_{i}=0$, and the governing equations recovers the form used for an inertial frame of reference. This acceleration is used to avoid excessive numerical oscillations that would eventually make the code unstable if the inital condition is the reference operating conditions. For an undisturbed flow condition $\mathbf{U}_{\infty}$, the pseudo body forces are given by [108]:

$$
\begin{aligned}
f_{x}^{*} & =\frac{f_{x}}{\rho_{\infty}\left(U_{\infty}\right)^{2} / L}=\frac{\rho\left(U_{\infty} \operatorname{sen}(\pi / 2-\beta) \cos (\alpha) / t_{a}\right)}{\rho_{\infty}\left(U_{\infty}\right)^{2} / L} \\
& =\left(\frac{\rho}{\rho_{\infty}}\right)\left(\frac{L / U_{\infty}}{t_{a}}\right) \operatorname{sen}(\pi / 2-\beta) \cos (\alpha) \\
& =\frac{\rho \operatorname{sen}(\pi / 2-\beta) \cos (\alpha)}{t_{a}}, \\
f_{y}^{*} & =\frac{f_{y}}{\rho_{\infty}\left(U_{\infty}\right)^{2} / L}=\frac{\rho\left(U_{\infty} \operatorname{sen}(\pi / 2-\beta) \operatorname{sen}(\alpha) / t_{a}\right)}{\rho_{\infty}\left(U_{\infty}\right)^{2} / L} \\
& =\left(\frac{\rho}{\rho_{\infty}}\right)\left(\frac{L / U_{\infty}}{t_{a}}\right) \operatorname{sen}(\pi / 2-\beta) \operatorname{sen}(\alpha) \\
& =\frac{\rho \operatorname{sen}(\pi / 2-\beta) \operatorname{sen}(\alpha)}{t_{a}}, \\
f_{z}^{*} & =\frac{f_{z}}{\rho_{\infty}\left(U_{\infty}\right)^{2} / L}=\frac{\rho\left(U_{\infty} \cos (\pi / 2-\beta) / t_{a}\right)}{\rho_{\infty}\left(U_{\infty}\right)^{2} / L} \\
& =\left(\frac{\rho}{\rho_{\infty}}\right)\left(\frac{L / U_{\infty}}{t_{a}}\right) \cos (\pi / 2-\beta) \\
& =\frac{\rho \operatorname{sen}(\beta)}{t_{a}} .
\end{aligned}
$$




\subsubsection{Numerical Method}

In order to be solved numerically using a finite-volume formulation, the governing equations are written in the following vector form:

$$
\frac{\partial \mathbf{U}}{\partial t}+\frac{\partial \mathbf{E}}{\partial x}+\frac{\partial \mathbf{F}}{\partial y}+\frac{\partial \mathbf{G}}{\partial z}=\mathbf{R}
$$

Defining tensor $\Pi$ as:

$$
\Pi=\mathbf{E} \otimes \mathbf{i}+\mathbf{F} \otimes \mathbf{j}+\mathbf{G} \otimes \mathbf{k},
$$

Equation (4.73) is rewritten as:

$$
\frac{\partial \mathbf{U}}{\partial t}+\nabla \cdot \Pi=\mathbf{R}
$$

For the Euler equations, the conservative variables vector $\mathbf{U}$, and flux vectors $\mathbf{E}, \mathbf{F}, \mathbf{G}$ are given by:

$$
\mathbf{U}=\left[\begin{array}{c}
\rho \\
\rho u \\
\rho v \\
\rho w \\
\rho e_{t}
\end{array}\right], \mathbf{E}=\left[\begin{array}{c}
\rho u \\
\rho u u+p \\
\rho u v \\
\rho u w \\
\left(\rho e_{t}+p\right) u
\end{array}\right], \mathbf{F}=\left[\begin{array}{c}
\rho v \\
\rho v u \\
\rho v v+p \\
\rho v w \\
\left(\rho e_{t}+p\right) v
\end{array}\right], \mathbf{G}=\left[\begin{array}{c}
\rho w \\
\rho w u \\
\rho w v \\
\rho w w+p \\
\left(\rho e_{t}+p\right) w
\end{array}\right] .
$$

The volume pseudo-force and pseudo-work vector $\mathbf{R}$ is given by:

$$
\mathbf{R}=\left[\begin{array}{c}
0 \\
f_{x} \\
f_{y} \\
f_{z} \\
f_{x} u+f_{y} v+f_{z} w
\end{array}\right]
$$

By integrating equation 4.75 over the control volume $V$, and applying the divergence theorem to the first term of the right-hand side, we obtain:

$$
\frac{\partial}{\partial t} \int_{V} \mathbf{U} d V=-\int_{V}(\nabla \cdot \Pi) d V+\int_{V} \mathbf{R} d V=-\oint_{S}(\Pi \cdot \mathbf{n}) d S+\int_{V} \mathbf{R} d V .
$$

Defining the volumetric mean of vectors $\overline{\mathbf{U}}$ and $\overline{\mathbf{R}}$ in the control volume $V$ as:

$$
\overline{\mathbf{U}} \equiv \frac{1}{V} \int_{V} \mathbf{U} d V, \quad \overline{\mathbf{R}} \equiv \frac{1}{V} \int_{V} \mathbf{R} d V
$$


equation (4.78) is written as:

$$
\frac{\partial \overline{\mathbf{U}}}{\partial t}=-\frac{1}{V} \oint_{S}(\Pi \cdot \mathbf{n}) d S+\overline{\mathbf{R}}
$$

Evaluating the previous equation for a hexahedral control volume yields:

$$
\begin{aligned}
\left(\frac{\partial \overline{\mathbf{U}}}{\partial t}\right)_{i, j, k}=-\frac{1}{V_{i, j, k}} & {\left[\int_{S_{i+1 / 2}}(\Pi \cdot \mathbf{n}) d S+\int_{S_{i-1 / 2}}(\Pi \cdot \mathbf{n}) d S+\right.} \\
& \int_{S_{j+1 / 2}}(\Pi \cdot \mathbf{n}) d S+\int_{S_{j-1 / 2}}(\Pi \cdot \mathbf{n}) d S+ \\
& \left.\int_{S_{k+1 / 2}}(\Pi \cdot \mathbf{n}) d S+\int_{S_{k-1 / 2}}(\Pi \cdot \mathbf{n}) d S\right]+\overline{\mathbf{R}}_{i, j, k}
\end{aligned}
$$

where $S_{i+1 / 2}, S_{i-1 / 2}, S_{j+1 / 2}, S_{j-1 / 2}, S_{k+1 / 2}$ e $S_{k-1 / 2}$ are the surfaces that define the hexahedral control volume and $S_{i+1 / 2}$ is the common surface between volume $(i, j, k)$ and volume $(i+$ $1, j, k)$.

Considering the value of tensor $\Pi$ as constant over each of the control surfaces, it is possible to write a finite-volume, discrete-time approximation of the previous equation as:

$$
\begin{aligned}
\frac{\Delta \overline{\mathbf{U}}_{i, j, k}}{\Delta t}=-\frac{1}{V_{i, j, k}} & (\Pi \cdot \mathbf{S})_{i+1 / 2}+(\Pi \cdot \mathbf{S})_{i-1 / 2}+ \\
& (\Pi \cdot \mathbf{S})_{j+1 / 2}+(\Pi \cdot \mathbf{S})_{j-1 / 2}+ \\
& \left.(\Pi \cdot \mathbf{S})_{k+1 / 2}+(\Pi \cdot \mathbf{S})_{k-1 / 2}\right]+\overline{\mathbf{R}}_{i, j, k}
\end{aligned}
$$

using time steps of size $\Delta t$.

Defining $\mathcal{F}_{i, j, k}$ as a function of the flux of tensor $\Pi$ over the surfaces of finite volume $V_{i, j, k}$ during time step $\Delta t$ as:

$$
\begin{array}{r}
\mathcal{F}_{i, j, k}=\frac{\Delta t}{V_{i, j, k}}\left[(\Pi \cdot \mathbf{S})_{i+1 / 2}+(\Pi \cdot \mathbf{S})_{i-1 / 2}+(\Pi \cdot \mathbf{S})_{j+1 / 2}+\right. \\
\left.(\Pi \cdot \mathbf{S})_{j-1 / 2}+(\Pi \cdot \mathbf{S})_{k+1 / 2}+(\Pi \cdot \mathbf{S})_{k-1 / 2}\right],
\end{array}
$$

and $\mathcal{R}_{i, j, k}$ as a function of the effect of body forces acting over the same finite volume during the same time step, we have:

$$
\mathcal{R}_{i, j, k}=\Delta t \cdot \overline{\mathbf{R}}_{i, j, k}
$$

a finite-volume, discrete-time approximation of equation 4.82 is given by:

$$
\Delta \overline{\mathbf{U}}_{i, j, k}=-\mathcal{F}_{i, j, k}+\mathcal{D}_{i, j, k}+\mathcal{R}_{i, j, k}
$$


In equation 4.85, the explicit artificial dissipation $\mathcal{D}_{i, j, k}$ is added in the right-hand side in order to control the discretization error generated by the calculation of the flux function $\mathcal{F}_{i, j, k}$

In order to advance equation 4.85 with a high temporal resolution a third-order accurate Runge-Kutta method [112] is used, resulting in the following three steps:

$$
\begin{gathered}
\overline{\mathbf{U}}_{i, j, k}^{1}=\overline{\mathbf{U}}_{i, j, k}^{n}-\left(\mathcal{F}_{i, j, k}^{n}-\mathcal{D}_{i, j, k}^{n}-\mathcal{R}_{i, j, k}^{n}\right], \\
\overline{\mathbf{U}}_{i, j, k}^{2}=\frac{3}{4} \overline{\mathbf{U}}_{i, j, k}^{n}+\frac{1}{4} \overline{\mathbf{U}}_{i, j, k}^{1}-\frac{1}{4}\left(\mathcal{F}_{i, j, k}^{1}-\mathcal{D}_{i, j, k}^{1}-\mathcal{R}_{i, j, k}^{1}\right), \\
\overline{\mathbf{U}}_{i, j, k}^{n+1}=\frac{1}{3} \overline{\mathbf{U}}_{i, j, k}^{n}+\frac{2}{3} \overline{\mathbf{U}}_{i, j, k}^{2}-\frac{2}{3}\left(\mathcal{F}_{i, j, k}^{2}-\mathcal{D}_{i, j, k}^{2}-\mathcal{R}_{i, j, k}^{2}\right) .
\end{gathered}
$$

In order to calculate $\mathcal{F}_{i, j, k}$ in any of the previous steps, the flux of tensor $\Pi$ over each of the control surfaces must be calculated. For the control surface $S_{i+1 / 2}$, this flux is given by:

$$
(\Pi \cdot \mathbf{S})_{i+1 / 2}=\left[\begin{array}{c}
(\Pi \cdot \mathbf{S})_{1} \\
(\Pi \cdot \mathbf{S})_{2} \\
(\Pi \cdot \mathbf{S})_{3} \\
(\Pi \cdot \mathbf{S})_{4} \\
(\Pi \cdot \mathbf{S})_{5}
\end{array}\right]_{i+1 / 2}
$$

The first component of the vector defined by the above equation is associated to the continuity equation and given by:

$$
(\Pi \cdot \mathbf{S})_{1}=\rho_{i+1 / 2}\left(q_{s}\right)_{i+1 / 2}
$$

where the volumetric flux is:

$$
\left(q_{s}\right)_{i+1 / 2}=\mathbf{u}_{i+1 / 2} \cdot \mathbf{S}_{i+1 / 2}=u_{i+1 / 2}\left(s_{x}\right)_{i+1 / 2}+v_{i+1 / 2}\left(s_{y}\right)_{i+1 / 2}+w_{i+1 / 2}\left(s_{z}\right)_{i+1 / 2} .
$$

The second, third, and fourth components are associated with the three components of the momentum equation and the fifth component is associated with the energy equation. For the Euler equations, those components are given by from equation 4.92 to equation 4.95:

$$
\begin{aligned}
& (\Pi \cdot \mathbf{S})_{2}=(\rho u)_{i+1 / 2}\left(q_{s}\right)_{i+1 / 2}+p_{i+1 / 2}\left(s_{x}\right)_{i+1 / 2}, \\
& (\Pi \cdot \mathbf{S})_{3}=(\rho v)_{i+1 / 2}\left(q_{s}\right)_{i+1 / 2}+p_{i+1 / 2}\left(s_{y}\right)_{i+1 / 2},
\end{aligned}
$$




$$
\begin{aligned}
& (\Pi \cdot \mathbf{S})_{4}=(\rho w)_{i+1 / 2}\left(q_{s}\right)_{i+1 / 2}+p_{i+1 / 2}\left(s_{z}\right)_{i+1 / 2}, \\
& (\Pi \cdot \mathbf{S})_{5}=\left(\rho e_{t}\right)_{i+1 / 2}\left(q_{s}\right)_{i+1 / 2}+p_{i+1 / 2}\left(q_{s}\right)_{i+1 / 2} .
\end{aligned}
$$

In order to calculate the flux $(\Pi \cdot \mathbf{S})$ according to equations 4.90 to 4.95 , it is necessary to approximate the values of the variables at the control surface $\mathbf{S}_{i+1 / 2}$ from the mean values of the conservative variables in the control volumes, given by the vector:

$$
\overline{\mathbf{U}}_{i, j, k}=\left[\begin{array}{c}
\bar{\rho} \\
\overline{\rho u} \\
\overline{\rho v} \\
\overline{\rho w} \\
\overline{\rho e_{t}}
\end{array}\right]_{i, j, k} .
$$

In order to obtain the momentum and energy primitive variables, the Favre mean is used to calculate the mass-averaged momentum and energy primitive variables as:

$$
\widetilde{u}=\frac{\overline{\rho u}}{\bar{\rho}}, \quad \widetilde{v}=\frac{\overline{\rho v}}{\bar{\rho}}, \quad \widetilde{w}=\frac{\overline{\rho w}}{\bar{\rho}}, \quad \widetilde{e_{t}}=\frac{\overline{\rho e_{t}}}{\bar{\rho}} .
$$

The mean of the total energy is given by:

$$
\widetilde{e_{t}}=\widetilde{e}+\widetilde{e_{k}}=\widetilde{e}+\frac{\widetilde{u} \widetilde{u}+\widetilde{v}+\widetilde{w} \widetilde{w}}{2}
$$

so the mean internal energy is calculated as:

$$
\widetilde{e}=\widetilde{e_{t}}-\widetilde{e_{k}}=\widetilde{e_{t}}-\frac{\widetilde{u} \widetilde{u}+\widetilde{v} \widetilde{v} \widetilde{w}}{2}
$$

and the mean pressure and temperature in the control volume is calculated using the state equation as:

$$
\widetilde{p}=(\gamma-1) \bar{\rho} \widetilde{e}, \quad \widetilde{T}=\frac{\gamma M_{\mathrm{ref}}^{2} \widetilde{p}}{\bar{\rho}}
$$

It is important to note that the first terms in the right-hand side of equations 4.90 , $4.92,4.93,4.94$, and 4.95 are the fluxes of mass, momentum and total energy through surface $\mathbf{S}_{i+1 / 2}$ and the other terms are fluxes that are functions of the right-hand sides of the momentum and total energy equations.

In order to calculate the fluxes of mass, momentum and total energy through surface $\mathbf{S}_{i+1 / 2}$, an approach for interpolating the values of the conservative and primitive variables 
at the control surfaces, while mantaining the spectral resolution and stability with a formal fourth order interpolation is proposed. Starting with the compact interpolation scheme [81]:

$$
\begin{aligned}
\beta f_{i-2}+\alpha f_{i-1}+ & f_{i}+\alpha f_{i+1}+\beta f_{i+2}= \\
& \frac{c}{2}\left(f_{i+5 / 2}-f_{i-5 / 2}\right)+\frac{b}{2}\left(f_{i+3 / 2}+f_{i-3 / 2}\right)+\frac{a}{2}\left(f_{i+1 / 2}-f_{i-1 / 2}\right),
\end{aligned}
$$

a general form for a centered compact numerical interpolation scheme with stencil sizes $N$ and $M$, for the left and right hand sides respectively, is given by:

$$
\sum_{m=0}^{M} \frac{\alpha_{m}}{2}\left(f_{i+m}+f_{i-m}\right)=\sum_{n=1}^{N} \frac{a_{n}}{2}\left(f_{i+n-1 / 2}+f_{i-n+1 / 2}\right) .
$$

By applying the following Taylor expansion to the above equation around the face $i$ location, we have:

$$
f(x)=\sum_{i=0}^{\infty} \frac{\partial^{i}}{\partial x^{i}} f\left(x_{0}\right) \frac{\left(x-x_{0}\right)^{i}}{i !},
$$

where for the left-hand side of equation 4.102 we have for regular cartesian meshes:

$$
\left(x-x_{0}\right)^{i}=( \pm \Delta x m)^{i}
$$

and for the right hand side of the same equation, we then have:

$$
\left(x-x_{0}\right)^{i}=[ \pm(\Delta x(n-1 / 2))]^{i},
$$

the expanded form of equation 4.102 is obtained as:

$$
\begin{aligned}
& \sum_{m=0}^{M} \frac{\alpha_{m}}{2}\left(\sum_{i=0}^{\infty} \frac{\partial^{i}}{\partial x^{i}} f\left(x_{0}\right) \frac{(\Delta x m)^{i}}{i !}+\sum_{i=0}^{\infty} \frac{\partial^{i}}{\partial x^{i}} f\left(x_{0}\right) \frac{(-\Delta x m)^{i}}{i !}\right)= \\
& \quad \sum_{n=1}^{N} \frac{a_{n}}{2}\left(\sum_{i=0}^{\infty} \frac{\partial^{i}}{\partial x^{i}} f\left(x_{0}\right) \frac{[\Delta x(n-1 / 2)]^{i}}{i !}+\sum_{i=0}^{\infty} \frac{\partial^{i}}{\partial x^{i}} f\left(x_{0}\right) \frac{[-(\Delta x(n-1 / 2))]^{i}}{i !}\right) .
\end{aligned}
$$

Rearranging equation 4.106 , the expanded form can be written as:

$$
\begin{aligned}
\sum_{m=0}^{M} \frac{\alpha_{m}}{2}\left(\sum_{i=0}^{\infty} \frac{\partial^{i}}{\partial x^{i}} f\left(x_{0}\right) \frac{1}{i !}\left[(\Delta x m)^{i}+(-\Delta x m)^{i}\right]\right)= \\
\quad \sum_{n=1}^{N} \frac{a_{n}}{2}\left(\sum_{i=0}^{\infty} \frac{\partial^{i}}{\partial x^{i}} f\left(x_{0}\right) \frac{1}{i !}\left[(\Delta x(n-1 / 2))^{i}+(-(\Delta x(n-1 / 2)))^{i}\right]\right)
\end{aligned}
$$

and it is automatically fulfilled for odd-order derivatives, since all the the terms in the brackets are equal to zero. 
Now we consider only the even terms for the previous equation:

$$
\sum_{m=0}^{M} \alpha_{m}\left(\sum_{i=0}^{\infty} \frac{\partial^{2 i}}{\partial x^{2 i}} f\left(x_{0}\right) \frac{1}{2 i !}(\Delta x m)^{2 i}\right)=\sum_{n=1}^{N} a_{n}\left(\sum_{i=0}^{\infty} \frac{\partial^{2 i}}{\partial x^{2 i}} f\left(x_{0}\right) \frac{1}{2 i !}(\Delta x(n-1 / 2))^{2 i}\right) .
$$

Using equation 4.108 , it is possible to build numerical schemes with the maximum possible precision by combining the same derivative order terms on the left and right hand sides. For each even formal order to be satisfied, its equation must be fulfilled for increasing even precision orders beginning from zero. Considering a regular Cartesian mesh with constant $\Delta x$, the maximum possible precision is given by:

$$
\sum_{m=0}^{M} \alpha_{m}(m)^{2 i}=\sum_{n=1}^{N} a_{n}(n-1 / 2)^{2 i} \text { on the order of }(\Delta x)^{2 i}
$$

For a numerical scheme to have the highest formal order, a $(M \times N)-1$ system of equations must be solved from the expression above to obtain all the coefficients $\alpha_{m}$ and $a_{n}$. Each equation will give a constraint to obtain a desired formal order. Note that the procedure above can also be used for derivatives regarding finite differences and filtering schemes for removal of short length scales.

For computational aeroacoustics, good wave propagation properties are desired for the numerical scheme and wave domain analysis is required for such. Applying the inverse Fourier transform:

$$
f(x)=\frac{1}{\sqrt{2 \pi}} \int_{-\infty}^{+\infty} F(k) e^{j 2 \pi k x} d k
$$

on equation 4.102, we obtain:

$$
\begin{gathered}
\sum_{m=0}^{M} \alpha_{m}\left(\int_{-\infty}^{+\infty} F(k)\left[e^{j 2 \pi k(x+\Delta x m)}+e^{j 2 \pi k(x-\Delta x m)}\right] d k\right)= \\
\sum_{n=1}^{N} a_{n}\left(\int_{-\infty}^{+\infty} \hat{F}(k)\left[e^{j 2 \pi k(x+\Delta x(m-1 / 2))}+e^{j 2 \pi k(x-\Delta x(m-1 / 2))}\right] d k\right) .
\end{gathered}
$$


By applying the Euler formula to the above equation, we have:

$$
\begin{gathered}
\sum_{m=0}^{M} \alpha_{m}\left(\int_{-\infty}^{+\infty} F(k)[\cos (2 \pi k \Delta x m)+j \sin (2 \pi k \Delta x m)\right. \\
\quad+\cos (-2 \pi k \Delta x(m+1 / 2))+j \sin (-2 \pi k \Delta x(m+1 / 2))] d k)= \\
\sum_{n=1}^{N} a_{n}\left(\int_{-\infty}^{+\infty} \hat{F}(k)[\cos (2 \pi k \Delta x(m-1 / 2))+j \sin (2 \pi k \Delta x(m-1 / 2))\right. \\
\quad+\cos (-2 \pi k \Delta x(m+1 / 2))+j \sin (-2 \pi k \Delta x(m+1 / 2))] d k)
\end{gathered}
$$

we then obtain the following relation:

$$
\int_{-\infty}^{+\infty} F(k) \sum_{m=0}^{M} \alpha_{m} \cos (2 \pi k \Delta x m) d k=\int_{-\infty}^{+\infty} \hat{F}(k) \sum_{n=1}^{N} a_{n} \cos (2 \pi k \Delta x(n-1 / 2)) d k .
$$

Defining a transfer function for each given wavenumber $k$ as:

$$
T(k)=\frac{F(k)}{\hat{F}(k)}=\frac{\sum_{n=1}^{N} a_{n} \cos (2 \pi k \Delta x(n-1 / 2))}{\sum_{m=0}^{M} \alpha_{m} \cos (2 \pi k \Delta x m)},
$$

it is possible to evaluate the numerical error associated with each wavenumber $k$, represented by the numerical solution along with the calculated interpolation coefficients $a_{n}$ and $\alpha_{m}$. The product $2 \pi k \Delta x$ is the phase angle associated with the number of points used to define a given wavenumber, where it covers the domain $(-\pi, \pi)$ for a given length $L$.

For transfer functions with value $T(k)=1$, there is no numerical error, i.e., wavenumber $k$ will propagate with no damping nor amplification. On the other hand, for transfer functions with values $T(k)<1$, the numerical error has damping characteristics and for $T(k)>1$, the numerical error associated to wavenumber $k$ has amplifying characteristics.

For a regular Cartesian grid with $x$-direction resolution $\Delta x$ and length $L$, the shortest wavelength that can be represented is $\lambda_{\min }=2 \Delta x$ and the largest one is $\lambda_{\max }=L$. For the associated wavenumber, $k=1 / \lambda$, the biggest wavenumber that can represented is $k_{\max }=1 /(2 \Delta x)$, while the smallest value is $k_{\min }=1 / L$. For a regular Cartesian mesh we have:

$$
\Delta x=\frac{L}{N_{v}},
$$


where $N_{v}$ if the number of finite volumes along the length $L$ and, therefore, the minimum wavenumber $k_{\min }$ is given by:

$$
k_{\min }=\frac{1}{N_{v} \Delta x} .
$$

The equation above can be regarded as the minimum wavenumber obtainable for the given mesh resolution.

Rearranging equation 4.116 and substituting into equation 4.114 we have:

$$
T(k)=\frac{\sum_{n=1}^{N} a_{n} \cos \left(\frac{2 \pi}{N_{v}}(n-1 / 2)\right)}{\sum_{m=0}^{M} \alpha_{m} \cos \left(\frac{2 \pi}{N_{v}} m\right)} .
$$

The expression obtained is a variation from equation 4.114 where the number of points is the variable to estimate the error of the lesser wavenumber $k$ that the given volume mesh can define.

For a given numerical interpolation stencil, compact or not, several assumptions can be made to determine its coefficients; equations 4.109 and 4.114 can be combined to produce schemes with desired formal precision and spectral characteristics. Tam[113] and Webb built explicit (non-compact) finite difference schemes (Dispersion-Relation-Preserving schemes) by using an optimization technique to achieve optimal spectral resolution using some of the stencil degrees of freedom and maintaining a formal precision order with the remaining ones. Lele[81] used a combination of implicit (compact) formal precision and non optimized spectral defined relations for finite difference and finite volume methods to achieve a "spectral-like" resolution scheme where a formal precision order and good spectral behavior were obtained.

In a previous work to the moving immersed boundary methodology, the skew-symmetric scheme from Ducros [114] was the one used, given by:

$$
f_{i+1 / 2, j, k}=\frac{2}{3}\left(f_{i, j, k}+f_{i+1, j, k}\right)-\frac{1}{12}\left(f_{i-1 . j . k}+f_{i, j, k}+f_{i+1, j, k}+f_{i+2, j, k}\right),
$$

for the surface interpolation of all the primitive and conservative variables. In this work, a fourth-order and non compact (explicit) interpolation scheme is proposed, using equation 
4.109 to obtain the stencil coefficients, by solving the following linear system:

$$
\begin{aligned}
a_{0} & =\alpha_{1}+\alpha_{2} \quad \text { on the order of }(\Delta x)^{0}, \\
0 & =\frac{1}{4} \alpha_{1}+\frac{9}{4} \alpha_{2} \quad \text { on the order of }(\Delta x)^{2} .
\end{aligned}
$$

Since $\alpha_{0}=1$ can be made arbitrarily, solving this linear system for $a_{1}$ and $a_{2}$ we obtain:

$$
\alpha_{1}=\frac{9}{8}, \quad \alpha_{2}=-\frac{1}{8}
$$

By substituting these coefficients in equation 4.102 we obtain the interpolation scheme:

$$
f_{i+1 / 2, j, k}=\frac{9}{16}\left(f_{i+1, j, k}+f_{i, j, k}\right)-\frac{1}{16}\left(f_{i+2, j, k}+f_{i-1, j, k,}\right) .
$$

Applying both schemes into equation 4.114, their spectral resolution can be assessed.

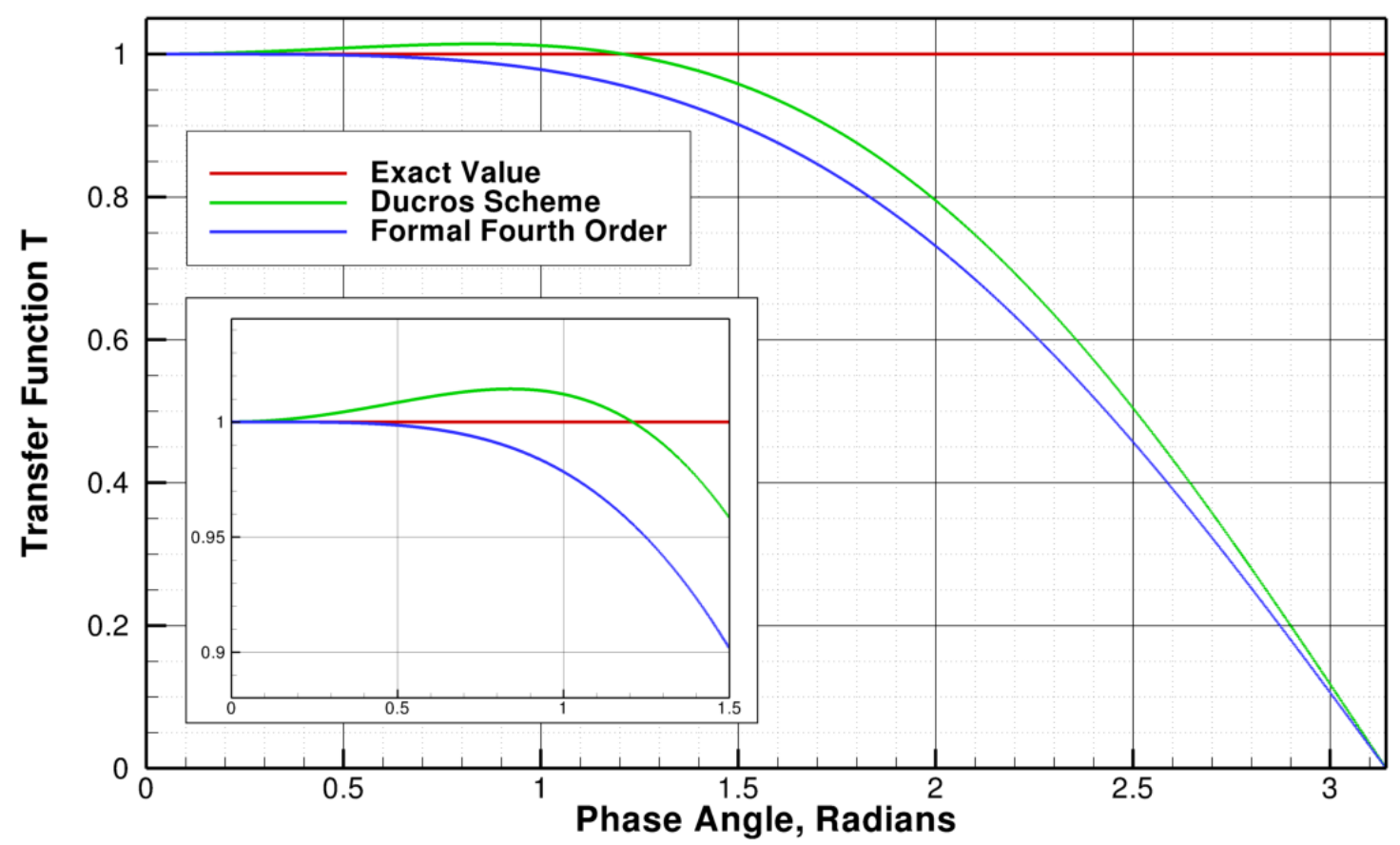

Figure 4.3: Spectral resolution of several numerical interpolation schemes. Note the amplification region from the Ducros' scheme, while the formal fourth order scheme has an asymptotic behavior around the unity value.

Like the dispersion relation preserving and spectral-like schemes, the former numerical scheme [114] used in VAT shows amplification (see figure 4.3) at some region of the spectral domain, indicating that at that region the code might be generating higher wavenumber content than there actually is from the exact solution. This introduces instabilities to the 
numerical calculation due to a non-physical amplification. A hypothesis that can be made about this instability case. The oscillation of the spectral behavior of the scheme around the unity value of the transfer function, brings instability to the discretization process and makes harder to achieve a stable and converged solution. When the maximum formal order is obtained for an interpolation scheme an asymptotically behavior around the unity value for the transfer function gives the best stability and higher precision order.

Even having less spectral accuracy, the formal fourth-order scheme still has great resolution for the cases presented in this work. For a second order spatial accuracy code, a wavelength is finely propagated by 35 points [115], or volumes considering the finite volume discretization. According to equation 4.117 and considering a second order formal explicit interpolation, where the interpolated value at surface $\mathbf{S}_{i+1 / 2}$ is the mean of the neighbouring volumes $\left(a_{1}=1\right.$ in equation 4.109), the transfer function has a value of $0.995975=T(N=35)$ or an error of about $0.4 \%$ of the exact value. Considering the simulation cases of this work, where we have 300 volumes along a characteristic length of 0.2393 meters, that gives about $8 * 10 E-04$ meters of volume width. For a sound wave traveling in the quiescent media at about 347 meters per second, dividing it by the volume width and by the human hearing upper threshold of $22 \mathrm{kHz}$, we have 19.7159 volumes, we round up to 20 control volumes to define the wave at $22 \mathrm{kHz}$. For the wavenumber to be calculated by using the proposed formal fourth order scheme at the highest frequency of human hearing and by using at least 20 volumes to define it, according to equation 4.117 we have a value for the transfer function of 0.999773 or a numerical error of about $0.02 \%$ for the worst resolved waves, concluding that the explicit fourth order formal accuracy interpolation scheme is more than enough to define with very high resolution the whole spectra of human hearing.

Now by applying the explicit formal fourth order scheme on the primitive variables we have:

$$
\begin{gathered}
u_{i+1 / 2}=\frac{9}{16}\left(\widetilde{u}_{i, j, k}+\widetilde{u}_{i+1, j, k}\right)-\frac{1}{16}\left(\widetilde{u}_{i-1, j, k}+\widetilde{u}_{i+2, j, k}\right)+O\left(\Delta x^{4}\right), \\
v_{i+1 / 2}=\frac{9}{16}\left(\widetilde{v}_{i, j, k}+\widetilde{v}_{i+1, j, k}\right)-\frac{1}{16}\left(\widetilde{v}_{i-1, j, k}+\widetilde{v}_{i+2, j, k}\right)+O\left(\Delta x^{4}\right), \\
w_{i+1 / 2}=\frac{9}{16}\left(\widetilde{w}_{i, j, k}+\widetilde{w}_{i+1, j, k}\right)-\frac{1}{16}\left(\widetilde{w}_{i-1, j, k}+\widetilde{w}_{i+2, j, k}\right)+O\left(\Delta x^{4}\right),
\end{gathered}
$$

and

$$
p_{i+1 / 2}=\frac{9}{16}\left(\widetilde{p}_{i, j, k}+\widetilde{p}_{i+1, j, k}\right)-\frac{1}{16}\left(\widetilde{p}_{i-1, j, k}+\widetilde{p}_{i+2, j, k}\right)+O\left(\Delta x^{4)}\right.
$$


In this work, the same scheme used for the primitive variables is also used for the conservative variables $\rho, \rho u, \rho v, \rho w$ and $\rho e_{T}$. Using this approach, the value of the conservatives variables at surface $\mathbf{S}_{i+1 / 2}$ is given by:

$$
\begin{gathered}
\rho_{i+1 / 2}=\frac{9}{16}\left(\bar{\rho}_{i, j, k}+\bar{\rho}_{i+1, j, k}\right)-\frac{1}{16}\left(\bar{\rho}_{i-1, j, k}+\bar{\rho}_{i+2, j, k}\right)+O\left(\Delta x^{4}\right), \\
(\rho u)_{i+1 / 2}=\frac{9}{16}\left(\overline{\rho u}_{i, j, k}+\overline{\rho u}_{i+1, j, k}\right)-\frac{1}{16}\left(\overline{\rho u}_{i-1, j, k}+\overline{\rho u}_{i+2, j, k}\right)+O\left(\Delta x^{4}\right), \\
(\rho v)_{i+1 / 2}=\frac{9}{16}\left(\overline{\rho v}_{i, j, k}+\overline{\rho v}_{i+1, j, k}\right)-\frac{1}{16}\left(\overline{\rho v}_{i-1, j, k}+\overline{\rho v}_{i+2, j, k}\right)+O\left(\Delta x^{4}\right), \\
(\rho w)_{i+1 / 2}=\frac{9}{16}\left(\overline{\rho w}_{i, j, k}+\overline{\rho w}_{i+1, j, k}\right)-\frac{1}{16}\left(\overline{\rho w}_{i-1, j, k}+\overline{\rho w}_{i+2, j, k}\right)+O\left(\Delta x^{4}\right),
\end{gathered}
$$

and

$$
\left(\rho e_{t}\right)_{i+1 / 2}=\frac{9}{16}\left({\overline{\rho e_{t i, j, k}}}+{\overline{\rho e_{t i+1, j, k}}}\right)-\frac{1}{16}\left({\overline{\rho e_{t-1, j, k}}}+{\overline{\rho e_{t i+2, j, k}}}\right)+O\left(\Delta x^{4}\right) .
$$

The scheme proposed from equation 4.122 to equation 4.130 is non-monotonic, and therefore, an explicit artificial viscosity was previously included in equation 4.85 . In this work we use the basic idea of the the artificial dissipation model [116] and some modifications were made to improve the numerical stability for rotor-stator interaction case, we have then the numerical dissipation operator:

$$
\mathcal{D}_{i, j, k}=\left(d_{i+1 / 2}-d_{i-1 / 2}\right)+\left(d_{j+1 / 2}-d_{j-1 / 2}\right)+\left(d_{k+1 / 2}-d_{k-1 / 2}\right)
$$

where for surface $\mathbf{S}_{i+1 / 2}$ we have:

$$
d_{i+1 / 2}=\epsilon_{i+1 / 2}^{(2)}\left[\overline{\mathbf{U}}_{i+1, j, k}-\overline{\mathbf{U}}_{i, j, k}\right]-\epsilon_{i+1 / 2}^{(4)}\left[\overline{\mathbf{U}}_{i+2, j, k}-3 \overline{\mathbf{U}}_{i+1, j, k}+3 \overline{\mathbf{U}}_{i, j, k}-\overline{\mathbf{U}}_{i-1, j, k}\right]
$$

The first and second terms of the right-hand side of equation 4.132 are the second-order and fourth-order dissipation operators, respectively, and the coefficients for both operators are:

$$
\epsilon_{i+1 / 2}^{(2)}=K^{(2)} \max \left(\Psi_{i}, \Psi_{i+1}\right), \quad \epsilon_{i+1 / 2}^{(4)}=\max \left[0,\left(K^{(4)}-\epsilon_{i+1 / 2}^{(2)}\right)\right]
$$

and the pressure based sensor $\Psi_{i}$, given by:

$$
\Psi_{i}=\frac{\left|\widetilde{p}_{i+1, j, k}-2 \widetilde{p}_{i, j, k}+\widetilde{p}_{i-1, j, k}\right|}{\left|\widetilde{p}_{i+1, j, k}\right|+\left|2 \widetilde{p}_{i, j, k}\right|+\left|\widetilde{p}_{i-1, j, k}\right|} .
$$


For a better numerical stability of the moving immersed boundary method, a vorticity based sensor,

$$
\left(\Psi_{\text {rot }}\right)_{i+1 / 2}=K_{\text {rot }}^{(2)} \cdot|\nabla \times \widetilde{\mathbf{u}}|_{i+1 / 2}
$$

and a divergent based sensor,

$$
\left(\Psi_{\mathrm{div}}\right)_{i+1 / 2}=K_{\mathrm{div}}^{(2)} \cdot|\nabla \cdot \widetilde{\mathbf{u}}|_{i+1 / 2}
$$

were proposed previously [111], substituting the pressure based sensor for the second order numerical dissipation quantity, resuting in:

$$
\epsilon_{i+1 / 2}^{(2)}=\max \left[\left(\Psi_{\text {rot }}\right)_{i+1 / 2},\left(\Psi_{\text {div }}\right)_{i+1 / 2}\right]
$$

The previous sensors are derived from the velocity field. In order to have an even better stability for the numerical scheme to handle the intense gradients and other non linearities in the rotor and stator cascades interaction region, two new sensors based on the thermodynamic field are proposed. The first one is a pressure gradient based sensor, given by:

$$
\left(\Psi_{\mathrm{prs}}\right)_{i+1 / 2}=K_{\mathrm{prs}}^{(2)} \cdot|\nabla \widetilde{p}|_{i+1 / 2}
$$

and the second sensor is based on the density, given by:

$$
\left(\Psi_{\mathrm{den}}\right)_{i+1 / 2}=K_{\mathrm{den}}^{(2)} \cdot|\nabla \bar{\rho}|_{i+1 / 2}
$$

by modifying the second order dissipation sensor, given by equation 4.137 , we have:

$$
\epsilon_{i+1 / 2}^{(2)}=\max \left[\left(\Psi_{\mathrm{rot}}\right)_{i+1 / 2},\left(\Psi_{\mathrm{div}}\right)_{i+1 / 2},\left(\Psi_{\mathrm{prs}}\right)_{i+1 / 2},\left(\Psi_{\mathrm{den}}\right)_{i+1 / 2}\right]
$$

In order to calculate the spatial derivatives from the hydrodynamic and thermodynamic solutions to be used in the artificial viscosity, the divergence theorem in the form proposed by Hirsch [23] is used. The integrating volume is not the same as the finite volume discretization used in the simulations. The control volume $V_{p}$ used for the calculation of derivatives states the order of spatial precision that the derivatives will have. By applying the divergence theorem on the velocity component $u$, we have:

$$
\int_{V_{p}}(\nabla u) d V_{p}=\oint_{S_{p}} u d \mathbf{s}_{p}
$$


Evaluating the integration for an hexaedric control volume, we have:

$$
\int_{V_{p}}\left[\left(\frac{\partial u}{\partial x}\right) \mathbf{i}+\left(\frac{\partial u}{\partial y}\right) \mathbf{j}+\left(\frac{\partial u}{\partial z}\right) \mathbf{k}\right] d V_{p}=\oint_{S_{p}}\left[\left(u d s_{p x}\right) \mathbf{i}+\left(u d s_{p y}\right) \mathbf{j}+\left(u d s_{p z}\right) \mathbf{k}\right],
$$

we may arrange the components to an explicit form of the derivatives at each direction:

$$
\int_{V_{p}}\left(\frac{\partial u}{\partial x_{i}}\right) d V_{p}=\int_{S_{p}} u d s_{p x_{i}}
$$

Now defining the volumetric mean of the derivative for the integrating control volume:

$$
\overline{\left(\frac{\partial u}{\partial x_{i}}\right)} \equiv \frac{1}{V_{p}} \int_{p}\left(\frac{\partial u}{\partial x_{i}}\right) d V_{p}
$$

By combining equations 4.143 and 4.144 we obtain:

$$
\overline{\left(\frac{\partial u}{\partial x_{i}}\right)}=\frac{1}{V_{p}} \int_{S_{p}} u d s_{p x_{i}}
$$

Defining the net flux of the velocity component $u$ through the surface vector component $i$ as:

$$
f(u)_{x_{i}} \equiv \int_{S_{p}} u d s_{p x_{i}},
$$

substituting into equation 4.145 we have:

$$
\overline{\left(\frac{\partial u}{\partial x_{i}}\right)_{i, j, k}}=\frac{1}{V_{p_{i, j, k}}}\left[f(u)_{x_{i}}\right]_{i, j, k}
$$

To maintain the derivative calculation with the same fourth order as the flux calculation, a four control volumes numerical stencil is used to calculate the derivatives as shown in figure 4.4. The derivatives are calculated at the vertex $i, j$ with equations 4.146 and 4.147. Following the same idea of the finite volume method we have, for example, the value of $f(u)_{x_{i}}$ to cartesian volume meshes in the $x$ direction:

$$
\begin{aligned}
{\left[f(u)_{x}\right]_{i, j, k} } & =\left[f(u)_{x}\right]_{i+1 / 2, j, k}+\left[f(u)_{x}\right]_{i+1 / 2, j-1, k}+\left[f(u)_{x}\right]_{i-1 / 2, j, k} \\
& +\left[f(u)_{x}\right]_{i-1 / 2, j-1, k}+\left[f(u)_{x}\right]_{i+1 / 2, j, k-1}+\left[f(u)_{x}\right]_{i+1 / 2, j-1, k-1} \\
& +\left[f(u)_{x}\right]_{i-1 / 2, j, k-1}+\left[f(u)_{x}\right]_{i-1 / 2, j-1, k-1} .
\end{aligned}
$$




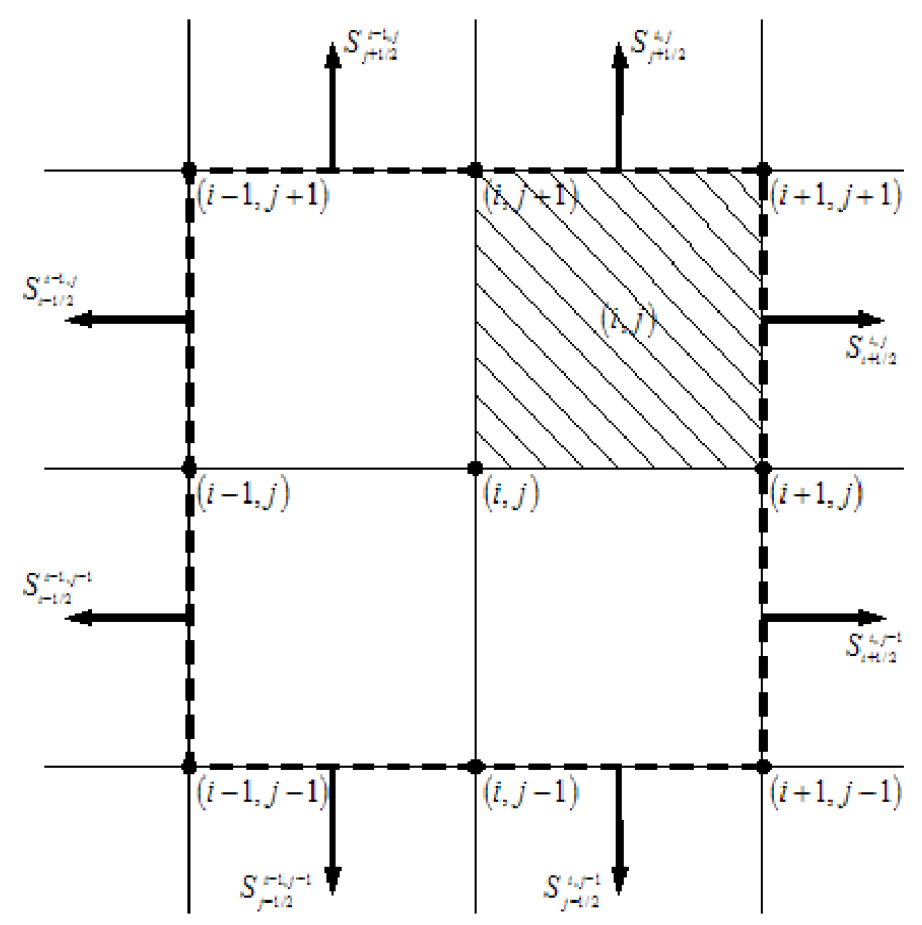

Figure 4.4: Mesh stencil for derivative calculation on vertex $i, j$ [117].

In the same way for $y$ and $z$ directions:

$$
\begin{aligned}
{\left[f(u)_{y}\right]_{i, j, k} } & =\left[f(u)_{y}\right]_{i, j+1 / 2, k}+\left[f(u)_{y}\right]_{i-1, j+1 / 2, k}+\left[f(u)_{y}\right]_{i, j-1 / 2, k} \\
& +\left[f(u)_{y}\right]_{i-1, j-1 / 2, k}+\left[f(u)_{y}\right]_{i, j+1 / 2, k-1}+\left[f(u)_{y}\right]_{i-1, j+1 / 2, k-1} \\
& +\left[f(u)_{y}\right]_{i, j-1 / 2, k-1}+\left[f(u)_{y}\right]_{i-1, j-1 / 2, k-1}
\end{aligned}
$$

and finally:

$$
\begin{aligned}
{\left[f(u)_{z}\right]_{i, j, k} } & =\left[f(u)_{z}\right]_{i, j, k+1 / 2}+\left[f(u)_{z}\right]_{i-1, j, k+1 / 2}+\left[f(u)_{z}\right]_{i, j, k-1 / 2} \\
& +\left[f(u)_{z}\right]_{i-1, j, k-1 / 2}+\left[f(u)_{z}\right]_{i, j-1, k+1 / 2}+\left[f(u)_{z}\right]_{i-1, j-1, k+1 / 2} \\
& +\left[f(u)_{z}\right]_{i, j-1 / 2, k-1 / 2}+\left[f(u)_{z}\right]_{i-1, j-1, k-1 / 2} .
\end{aligned}
$$

Each term of the previous equation may be obtained with the face values calculated with arithmetic mean:

$$
\left[f(u)_{x}\right]_{i+1 / 2, j, k}=u_{i+1 / 2, j, k}\left(\mathbf{i} \cdot \mathbf{S}_{x}\right)_{i+1 / 2, j, k}
$$


and in the other directions:

$$
\begin{aligned}
& {\left[f(u)_{y}\right]_{i, j+1 / 2, k}=u_{i, j+1 / 2, k}\left(\mathbf{j} \cdot \mathbf{S}_{y}\right)_{i, j+1 / 2, k},} \\
& {\left[f(u)_{z}\right]_{i, j, k+1 / 2}=u_{i, j, k+1 / 2}\left(\mathbf{k} \cdot \mathbf{S}_{z}\right)_{i, j, k+1 / 2}}
\end{aligned}
$$

where the face values may be evaluated through arithmetic mean from the volumes that share the same face:

$$
u_{i+1 / 2, j, k}=\frac{1}{2}\left(\widetilde{u}_{i, j, k}+\widetilde{u}_{i+1, j, k}\right) .
$$

Now with the derivative values calculated for the vertex $i, j, k$, the derivative values for the faces may again be calculated through arithmetic mean from the values in the vertexes:

$$
\begin{aligned}
\overline{\left(\frac{\partial u}{\partial x}\right)_{i+1 / 2, j, k+1 / 2}=} & \frac{1}{4}\left[\overline{\left(\frac{\partial u}{\partial x}\right)_{i+1, j, k}}+\overline{\left(\frac{\partial u}{\partial x}\right)_{i+1, j+1, k}}\right. \\
& \left.+\overline{\left(\frac{\partial u}{\partial x}\right)_{i+1, j, k+1}}+\overline{\left(\frac{\partial u}{\partial x}\right)_{i+1, j+1, k+1}}\right],
\end{aligned}
$$

where the indexes on the left hand side are evaluated on the faces and the indexes on the right hand side are evaluated at the vertexes. By doing this, the derivatives on the faces of the control volumes may be calculated and then applied on the artificial dissipation scheme to stabilize the code. Since the calculation of the vorticity and divergence of the velocity field, as well as the temperature and density gradients is fourth-order accurate in space, the resulting numerical method is also fourth-order accurate in space and third-order accurate in time.

\subsubsection{Numerical Imposition of Moving Surfaces by Using the Im- mersed Boundary Method}

The approach used in this work for imposing the boundary conditions at the boundary volumes, defined as the control volumes that contain one or more surface-grid points, is a discrete forcing one where the boundary conditions are imposed directly to the boundary volumes. 
In all the control volumes, the mean values of the conservative variables are given by:

$$
\overline{\mathbf{U}}_{i, j, k}=\left[\begin{array}{c}
\bar{\rho} \\
\overline{\rho u} \\
\overline{\rho v} \\
\overline{\rho w} \\
\overline{\rho e_{t}}
\end{array}\right]_{i, j, k}=\left[\begin{array}{c}
\bar{\rho} \\
\bar{\rho} \widetilde{u} \\
\bar{\rho} \widetilde{v} \\
\bar{\rho} \widetilde{w} \\
\bar{\rho} \widetilde{e_{t}}
\end{array}\right]_{i, j, k}
$$

In order to impose the free-slip condition in the boundary volumes for the Euler equations, the velocity of the fluid at the solid surface is decomposed in the normal (subscript $n$ ) and tangential (subscript $t$ ) components as:

$$
\widetilde{\mathbf{u}}=\widetilde{\mathbf{u}}_{n}+\widetilde{\mathbf{u}}_{t}=\widetilde{u}_{n} \mathbf{n}+\widetilde{u}_{t} \mathbf{t}
$$

Since the impenetrability condition in the boundary volumes establishes that the normal component of the fluid velocity must be equal to the normal component of the velocity of the moving body, $u_{n_{B}}$, the normal and tangential components of the velocity in the boundary volumes are given by:

$$
\widetilde{u}_{n}=u_{n}^{b}, \quad \text { e } \quad \widetilde{u}_{t} \neq 0
$$

Is is important to note that for a static surface, $u_{n_{B}}=0$, and for a moving surface the velocity of the moving bodies is equal to the normal component of the velocity of the surface for every point of the solid walls, given by $u_{n_{B}}=\mathbf{v}_{\text {rotor }} \cdot \mathbf{n}$.

For the Euler equations there is no momentum diffusion in the normal direction at the solid walls of the surfaces, yielding:

$$
\frac{\partial \widetilde{\mathbf{u}}}{\partial x_{n}}=0
$$

The boundary condition for the static pressure at the wall is obtained considering the momentum equation in the normal direction,

$$
\frac{\partial}{\partial t}\left(\bar{\rho} \widetilde{u}_{n}\right)+\frac{\partial}{\partial x_{n}}\left(\bar{\rho} \widetilde{u}_{n} \widetilde{u}_{n}\right)+\frac{\partial}{\partial x_{t}}\left(\bar{\rho} \widetilde{u}_{n} \widetilde{u}_{t}\right)=-\frac{\partial \widetilde{p}}{\partial x_{n}}
$$

where the expansion of all the terms of the previous equation results in:

$\widetilde{u}_{n} \frac{\partial \bar{\rho}}{\partial t}+\bar{\rho} \frac{\partial \widetilde{u}_{n}}{\partial t}+\left(\widetilde{u}_{n}\right)^{2} \frac{\partial \bar{\rho}}{\partial x_{n}}+\left(2 \bar{\rho} \widetilde{u}_{n}\right) \frac{\partial \widetilde{u}_{n}}{\partial x_{n}}+\left(\widetilde{u}_{n} \widetilde{u}_{t}\right) \frac{\partial \bar{\rho}}{\partial x_{t}}+\left(\bar{\rho} \widetilde{u}_{t}\right) \frac{\partial \widetilde{u}_{n}}{\partial x_{t}}+\left(\bar{\rho} \widetilde{u}_{n}\right) \frac{\partial \widetilde{u}_{t}}{\partial x_{t}}=-\frac{\partial \widetilde{p}}{\partial x_{n}}$. 
By Appling the imprenetrability condition to the momentum equation in the normal direction, we have:

$$
u_{n}^{b} \frac{\partial \bar{\rho}}{\partial t}+\bar{\rho} \frac{\partial u_{n}^{b}}{\partial t}+\left(u_{n}^{b}\right)^{2} \frac{\partial \bar{\rho}}{\partial x_{n}}+\left(2 \bar{\rho} u_{n}^{b}\right) \frac{\partial u_{n}^{b}}{\partial x_{n}}+\left(u_{n}^{b} u_{t}\right) \frac{\partial \bar{\rho}}{\partial x_{t}}+\left(\bar{\rho} u_{t}\right) \frac{\partial u_{n}^{b}}{\partial x_{t}}+\left(\bar{\rho} u_{n}^{b}\right) \frac{\partial u_{t}}{\partial x_{t}}=-\frac{\partial p}{\partial x_{n}} .
$$

For a static solid body, $u_{n}^{b}=0$, and the above equation yields:

$$
\frac{\partial \widetilde{p}}{\partial x_{n}}=0
$$

For a moving body, $u_{n_{B}} \neq 0$, if measured by an observer in an inertial system that is stationary. However, static pressure is independent from the velocity of the inertial system of the observer and if the later is moving with the same constant velocity of the moving surface it will also result in $u_{n_{B}}=0$, and using equation 4.163 will also result in:

$$
\frac{\partial \widetilde{p}}{\partial x_{n}}=0
$$

Considering the walls of the moving and static bodies are adiabatic, the boundary condition for the temperature is given by:

$$
\frac{\partial \widetilde{T}}{\partial x_{n}}=0
$$

and differentiating the state equation for an ideal gas with respect to the normal direction,

$$
\frac{\partial \widetilde{p}}{\partial x_{n}}=\frac{\partial}{\partial x_{n}}(\bar{\rho} \mathcal{R} \widetilde{T})=\mathcal{R} \bar{\rho} \frac{\partial \widetilde{T}}{\partial x_{n}}+\mathcal{R} \widetilde{T} \frac{\partial \bar{\rho}}{\partial x_{n}},
$$

results in:

$$
\frac{\partial \bar{\rho}}{\partial x_{n}}=0
$$

Since

$$
\widetilde{e}=\frac{1}{\gamma(\gamma-1) M_{\mathrm{ref}}^{2} \widetilde{T}},
$$

the adiabatic wall condition results in:

$$
\frac{\partial \widetilde{e}}{\partial x_{n}}=0
$$

Since $\mathbf{n}$ is a unit vector, with direction normal to the body wall in and outward sense and cartesian components $\mathbf{n}=n_{x} \mathbf{i}+n_{y} \mathbf{j}+n_{z} \mathbf{k}$, the derivatives of the flow variables $\widetilde{\mathbf{u}}, \widetilde{u}_{n}$, $\bar{\rho}$ and $\widetilde{e}$ in the normal direction can be evaluated as:

$$
\frac{\partial \widetilde{\mathbf{u}}}{\partial x_{n}}=\frac{\partial \widetilde{\mathbf{u}}}{\partial n}=\frac{\partial \widetilde{\mathbf{u}}}{\partial x} \frac{\partial x}{\partial n}+\frac{\partial \widetilde{\mathbf{u}}}{\partial y} \frac{\partial y}{\partial n}+\frac{\partial \widetilde{\mathbf{u}}}{\partial z} \frac{\partial z}{\partial n}=n_{x} \frac{\partial \widetilde{\mathbf{u}}}{\partial x}+n_{y} \frac{\partial \widetilde{\mathbf{u}}}{\partial y}+n_{z} \frac{\partial \widetilde{\mathbf{u}}}{\partial z},
$$




$$
\begin{aligned}
& \frac{\partial \bar{\rho}}{\partial x_{n}}=\frac{\partial \bar{\rho}}{\partial n}=\frac{\partial \bar{\rho}}{\partial x} \frac{\partial x}{\partial n}+\frac{\partial \bar{\rho}}{\partial y} \frac{\partial y}{\partial n}+\frac{\partial \bar{\rho}}{\partial z} \frac{\partial z}{\partial n}=n_{x} \frac{\partial \bar{\rho}}{\partial x}+n_{y} \frac{\partial \bar{\rho}}{\partial y}+n_{z} \frac{\partial \bar{\rho}}{\partial z} \\
& \frac{\partial \widetilde{e}}{\partial x_{n}}=\frac{\partial \widetilde{e}}{\partial n}=\frac{\partial \widetilde{e}}{\partial x} \frac{\partial x}{\partial n}+\frac{\partial \widetilde{e}}{\partial y} \frac{\partial y}{\partial n}+\frac{\partial \widetilde{e}}{\partial z} \frac{\partial z}{\partial n}=n_{x} \frac{\partial \widetilde{e}}{\partial x}+n_{y} \frac{\partial \widetilde{e}}{\partial y}+n_{z} \frac{\partial \widetilde{e}}{\partial z}
\end{aligned}
$$

Depending on the resolution of the cartesian and surface grids, more than one surface point can lie within a boundary volume and, in this case, the mean among all normal unit vectors associated to the grid points that lie within the boundary volume is used.

The derivatives in the normal direction of the boundary volumes (superscript $b$ ) can be evaluated according to the boundary conditions given by equations 4.160, 4.168, 4.170 as:

$$
\begin{aligned}
0 & =n_{x}\left(\frac{\partial \widetilde{\mathbf{u}}}{\partial x}\right)_{i, j, k}^{b}+n_{y}\left(\frac{\partial \widetilde{\mathbf{u}}}{\partial y}\right)_{i, j, k}^{b}+n_{z}\left(\frac{\partial \widetilde{\mathbf{u}}}{\partial z}\right)_{i, j, k}^{b}, \\
0 & =n_{x}\left(\frac{\partial \bar{\rho}}{\partial x}\right)_{i, j, k}^{b}+n_{y}\left(\frac{\partial \bar{\rho}}{\partial y}\right)_{i, j, k}^{b}+n_{z}\left(\frac{\partial \bar{\rho}}{\partial z}\right)_{i, j, k}^{b}, \\
0 & =n_{x}\left(\frac{\partial \widetilde{e}}{\partial x}\right)_{i, j, k}^{b}+n_{y}\left(\frac{\partial \widetilde{e}}{\partial y}\right)_{i, j, k}^{b}+n_{z}\left(\frac{\partial \widetilde{e}}{\partial z}\right)_{i, j, k}^{b} .
\end{aligned}
$$

If $n_{x}>0$, in the regular region of the Cartesian grid the derivative $\frac{\partial \rho}{\partial x}$ in the boundary volumes can be calculated with fourth order spatial precision using a forward finitedifference approach obtained from polynomial fitting proposed by Tanehill [118], given by:

$$
\left(\frac{\partial \bar{\rho}}{\partial x}\right)_{i, j, k}^{b}=\frac{1}{12 \Delta x}\left[-25 \bar{\rho}_{i, j, k}^{b}+48 \bar{\rho}_{i+1, j, k}-36 \bar{\rho}_{i+2, j, k}+16 \bar{\rho}_{i+3, j, k}-3 \bar{\rho}_{i+4, j, k}+O(\Delta x)^{4}\right] .
$$

Defining the difference operator:

$$
\begin{aligned}
D_{i}^{+} \bar{\rho} & =\frac{1}{25}\left(48 \bar{\rho}_{i+1, j, k}-36 \bar{\rho}_{i+2, j, k}+16 \bar{\rho}_{i+3, j, k}-3 \bar{\rho}_{i+4, j, k}\right) \\
D_{j}^{+} \bar{\rho} & =\frac{1}{25}\left(48 \bar{\rho}_{i, j+1, k}-36 \bar{\rho}_{i, j+2, k}+16 \bar{\rho}_{i, j+3, k}-3 \bar{\rho}_{i, j+4, k}\right) \\
D_{k}^{+} \bar{\rho} & =\frac{1}{25}\left(48 \bar{\rho}_{i, j, k+1}-36 \bar{\rho}_{i, j, k+2}+16 \bar{\rho}_{i, j, k+3}-3 \bar{\rho}_{i, j, k+4}\right) \\
D_{i}^{-} \bar{\rho} & =\frac{1}{25}\left(48 \bar{\rho}_{i-1, j, k}-36 \bar{\rho}_{i-2, j, k}+16 \bar{\rho}_{i-3, j, k}-3 \bar{\rho}_{i-4, j, k}\right) \\
D_{j}^{-} \bar{\rho} & =\frac{1}{25}\left(48 \bar{\rho}_{i, j-1, k}-36 \bar{\rho}_{i, j-2, k}+16 \bar{\rho}_{i, j-3, k}-3 \bar{\rho}_{i, j-4, k}\right) \\
D_{k}^{-} \bar{\rho} & =\frac{1}{25}\left(48 \bar{\rho}_{i, j, k-1}-36 \bar{\rho}_{i, j, k-2}+16 \bar{\rho}_{i, j, k-3}-3 \bar{\rho}_{i, j, k-4}\right)
\end{aligned}
$$

Equation 4.177 is written as:

$$
\left(\frac{\partial \bar{\rho}}{\partial x}\right)_{i, j, k}=\frac{25}{12 \Delta x}\left[-\bar{\rho}_{i, j, k}^{b}+D_{i}^{+} \bar{\rho}+O(\Delta x)^{4}\right] .
$$


If $\mathbf{n}=\mathbf{i}\left(n_{x}=1, n_{y}=0\right.$ and $\left.n_{z}=0\right)$, equation 4.175 gives:

$$
0=\left(\frac{\partial \bar{\rho}}{\partial x}\right)_{i, j, k}^{b},
$$

and introducing this result in equation 4.184 we have:

$$
\bar{\rho}_{i, j, k}^{b}=D_{i}^{+} \bar{\rho}+O(\Delta x)^{4} .
$$

Following the same line of reasoning, if $\mathbf{n}=\mathbf{j}\left(n_{x}=0, n_{y}=1\right.$ and $\left.n_{z}=0\right)$,

$$
\bar{\rho}_{i, j, k}^{b}=D_{j}^{+} \bar{\rho}+O(\Delta y)^{4},
$$

and if $\mathbf{n}=\mathbf{k}\left(n_{x}=0, n_{y}=0\right.$ e $\left.n_{z}=1\right)$,

$$
\bar{\rho}_{i, j, k}^{b}=D_{k}^{+} \bar{\rho}+O(\Delta z)^{4} .
$$

For the generalized case, where $\mathbf{n}=n_{x} \mathbf{i}+n_{y} \mathbf{j}+n_{z} \mathbf{k}$, the averaged density is calculated in the boundary volumes as the weighted value from the normal vector components magnitude $\left|n_{x}\right|,\left|n_{y}\right|$ e $\left|n_{z}\right|$,

$$
\bar{\rho}_{i, j, k}^{b}=\frac{\left|n_{x}\right| D_{i} \bar{\rho}+\left|n_{y}\right| D_{j} \bar{\rho}+\left|n_{z}\right| D_{k} \bar{\rho}}{\left|n_{x}\right|+\left|n_{y}\right|+\left|n_{z}\right|} .
$$

Following an analogous procedure, since $\partial \bar{\rho} / \partial x_{n}=\partial \widetilde{e} / \partial x_{n}=\partial \widetilde{\mathbf{u}} / \partial x_{n}=0$, the averaged internal energy and tangential velocity are calculated as the weighted values:

$$
\begin{gathered}
\widetilde{e}_{i, j, k}^{b}=\frac{\left|n_{x}\right| D_{i} \widetilde{e}+\left|n_{y}\right| D_{j} \widetilde{e}+\left|n_{z}\right| D_{k} \widetilde{e}}{\left|n_{x}\right|+\left|n_{y}\right|+\left|n_{z}\right|}, \\
\widetilde{\mathbf{u}}_{i, j, k}^{b}=\frac{\left|n_{x}\right| D_{i} \widetilde{\mathbf{u}}+\left|n_{y}\right| D_{j} \widetilde{\mathbf{u}}+\left|n_{z}\right| D_{k} \widetilde{\mathbf{u}}}{\left|n_{x}\right|+\left|n_{y}\right|+\left|n_{z}\right|} .
\end{gathered}
$$

In the previous equations, the difference operators $\left(D_{i}, D_{j}\right.$ and $\left.D_{k}\right)$ can be in the forward direction $\left(D_{i}^{+}, D_{j}^{+}\right.$and $\left.D_{k}^{+}\right)$, if the values of $n_{x}, n_{y}$ and $n_{z}$ are positive, or in the backward direction $\left(D_{i}^{-}, D_{j}^{-}\right.$and $\left.D_{k}^{-}\right)$, if the values of $n_{x}, n_{y}$ and $n_{z}$ are negative.

Since the normal velocity, $\widetilde{\mathbf{u}}_{n}$, is known for every boundary volume its cartesian components are also known and given by

$$
\left(\widetilde{\mathbf{u}}_{n}\right)_{i, j, k}^{b}=\left(\widetilde{u}_{n_{x}} \mathbf{i}+\widetilde{u}_{n_{y}} \mathbf{j}+\widetilde{u}_{n_{z}} \mathbf{k}\right)_{i, j, k}^{b}
$$

and since

$$
\widetilde{\mathbf{u}}_{i, j, k}^{b}=(\widetilde{\mathbf{u}})_{i, j, k}^{b}=\left(\widetilde{\mathbf{u}}_{n}\right)_{i, j, k}^{b}+\left(\widetilde{\mathbf{u}}_{t}\right)_{i, j, k}^{b},
$$


the cartesian components of of the tangential velocity, $\widetilde{\mathbf{u}}_{t}$, are given by:

$$
\left(\widetilde{u}_{t_{x}}\right)_{i, j, k}^{b}=\left(\widetilde{u}-\widetilde{u}_{n_{x}}\right)_{i, j, k}^{b}, \quad\left(\widetilde{u}_{t_{y}}\right)_{i, j, k}^{b}=\left(\widetilde{v}-\widetilde{u}_{n_{y}}\right)_{i, j, k}^{b}, \quad\left(\widetilde{u}_{t_{z}}\right)_{i, j, k}^{b}=\left(\widetilde{w}-\widetilde{u}_{n_{z}}\right)_{i, j, k}^{b}
$$

In this manner, the conservative variables vector for the boundary volumes are given by

$$
\overline{\mathbf{U}}_{i, j, k}^{b}=\left[\begin{array}{c}
\bar{\rho} \\
\bar{\rho}\left(\widetilde{u}_{t_{x}}+\widetilde{u}_{n_{x}}\right) \\
\bar{\rho}\left(\widetilde{u}_{t_{y}}+\widetilde{u}_{n_{y}}\right) \\
\bar{\rho}\left(\widetilde{u}_{t_{z}}+\widetilde{u}_{n_{z}}\right) \\
\bar{\rho}\left[\widetilde{e}+\frac{1}{2}\left[\left(\widetilde{u}_{t_{x}}+\widetilde{u}_{n_{x}}\right)^{2}+\left(\widetilde{u}_{t_{y}}+\widetilde{u}_{n_{y}}\right)^{2}+\left(\widetilde{u}_{t_{z}}+\widetilde{u}_{n_{z}}\right)^{2}\right]\right]
\end{array}\right]_{i, j, k}^{b} .
$$

\subsubsection{Expansional Viscosity Model Implementation in the VAT Code}

In this work, a first implementation of the expansional viscosity model is done into the VAT code. The numerical formulation is exactly the same from the previous section for the Euler equation. According to equation 4.19, the physical parameter $\Psi$ is related to the ratio of the flow characteristic time and the time relaxation related to the thermodynamic equilibrium of the fluid. We will consider that the relaxation time of the fluid is much smaller than the flow characteristic time:

$$
\frac{L_{0}}{U_{0}} \gg \tau
$$

as a consequence:

$$
\Psi \gg 1
$$

The above relation implies that in equation 4.19 the left hand side, the time rate term of change of the expansional viscosity coefficient, is negligible when compared with the right hand side. The consequence of this statement is that the expansional viscosity coefficient evolution is practically instantaneous. As a consequence the $\kappa$ term is only dependent on the fluid density:

$$
\kappa^{*}\left(\rho^{*}\right)=\rho^{*}\left(r_{M}^{2}-1\right)
$$

By adding the expansional viscosity model into the Euler equation and the work done by the expansional viscosity into the energy equation, the flux vectors 4.76 used on the VAT 
code will be in the following form (non dimensional superscript * already supressed):

$$
\begin{gathered}
\mathbf{U}=\left[\begin{array}{c}
\rho \\
\rho u \\
\rho v \\
\rho w \\
\rho e_{t}
\end{array}\right], \mathbf{E}=\left[\begin{array}{c}
\rho u \\
\rho u u-\sigma_{x x} \\
\rho u v \\
\rho u w \\
\rho v \\
\left(\rho e_{t}-\sigma_{x x}\right) u
\end{array}\right], \\
\mathbf{F}=\left[\begin{array}{c}
\rho w \\
\rho v v-\sigma_{y y} \\
\rho v w \\
\rho w u \\
\rho w v \\
\left(\rho e_{t}-\sigma_{y y}\right) v
\end{array}\right], \mathbf{G}=\left[\begin{array}{c} 
\\
\rho w w-\sigma_{z z} \\
\left(\rho e_{t}-\sigma_{z z}\right) w
\end{array}\right],
\end{gathered}
$$

where:

$$
\sigma_{x x}=\sigma_{y y}=\sigma_{z z}=-p_{0}+\left[\frac{\left(r_{M}^{2}-1\right)}{R e_{\kappa}}\right] \rho \nabla \cdot \mathbf{u} .
$$

Finally, we may define the following physical parameter:

$$
k_{\text {exp }}=\frac{\left(r_{M}^{2}-1\right)}{R e_{\kappa}} .
$$

\subsubsection{VAT Code Implementation Details}

As stated in chapter 1, parallel computing has been in use for several decades, mainly in high performance computing. Currently almost every electronic with computing capabilities, has central processing units with multiple processing cores. The main reason for using multiple cores is that the growth of the clock speed reached its physical limitations, and it was not feasible to increase the processing capability by simply increasing the clock speed. As a result, since around 2005 [119] computer CPUs implemented more cores with a single processor while the clock speed was held constant. Even with dozens of cores in the CPUs, the relative parallelism from the GPUs is far superior. With thousands of cores, the graphic units has parallel processing as their main processing characteristic, and as a consequence, fulfilling Moore's law in computational power.

The use of GPUs for parallel processing in high performance computing initially was impractical. With the advent of the CUDA architecture in 2007 [27], a new environment for 
the development of this type of application was more suitable to scientific and engineering applications with GPUs. At the end of 2009 the first CUDA Fortran compiler was available [30], where language extensions where added for the Fortran language, including memory management over the GPU memory.

The CUDA computing architecture is an hybrid programming model, i.e., some code parts may be executed both on CPU and GPU. Parts of the CUDA Fortran code may be implemented incrementally from the CPU to the GPU for a faster execution. The parallel levels of execution are usually more aggressive when the numerical work is exclusively run on the GPU. Subroutines that run in a GPU may execute more execution threads than on a CPU for the same time. These subroutines are called kernels. A call for a kernel specifies how many parallel instances of this kernel will be simultaneously executed. Each instance will be executed by a single thread. By organizing the execution threads into thread blocks, each thread has a single global index from the computation grid and a local index from its execution block. By using these indexes, execution loops may be executed simultaneously with the launch of a single block into one of the dozens of streaming multiprocessors from the GPU until all the grid blocks are processed.

Being an extremely parallel friendly methodology, the VAT numerical scheme may be implemented with high gains of performance when compared with its execution on CPUs. The usual performance gain with several GPUs with the same numerical methodology, when compared with the serial execution on a single core of CPU was around one hundred times faster.

\subsubsection{The GPU Architecture}

The GPU architecture, specially the NVIDIA branded ones, are built around an scalar array of streaming multiprocessors (SM). Each SM of a GPU is made to support the concurrent executions of hundreds of threads. From the available SMs of a single, it is possible to process thousands of threads at the same time.

When a subroutine (kernel) to be executed in a GPU is called, each thread block, which together constitutes an execution grid, are launched for a SM available for execution. Once scheduled for a SM, the threads of the block are then processed. Several blocks of a grid may 
be scheduled for a single SM that will be processed according to the resources availability of that SM.

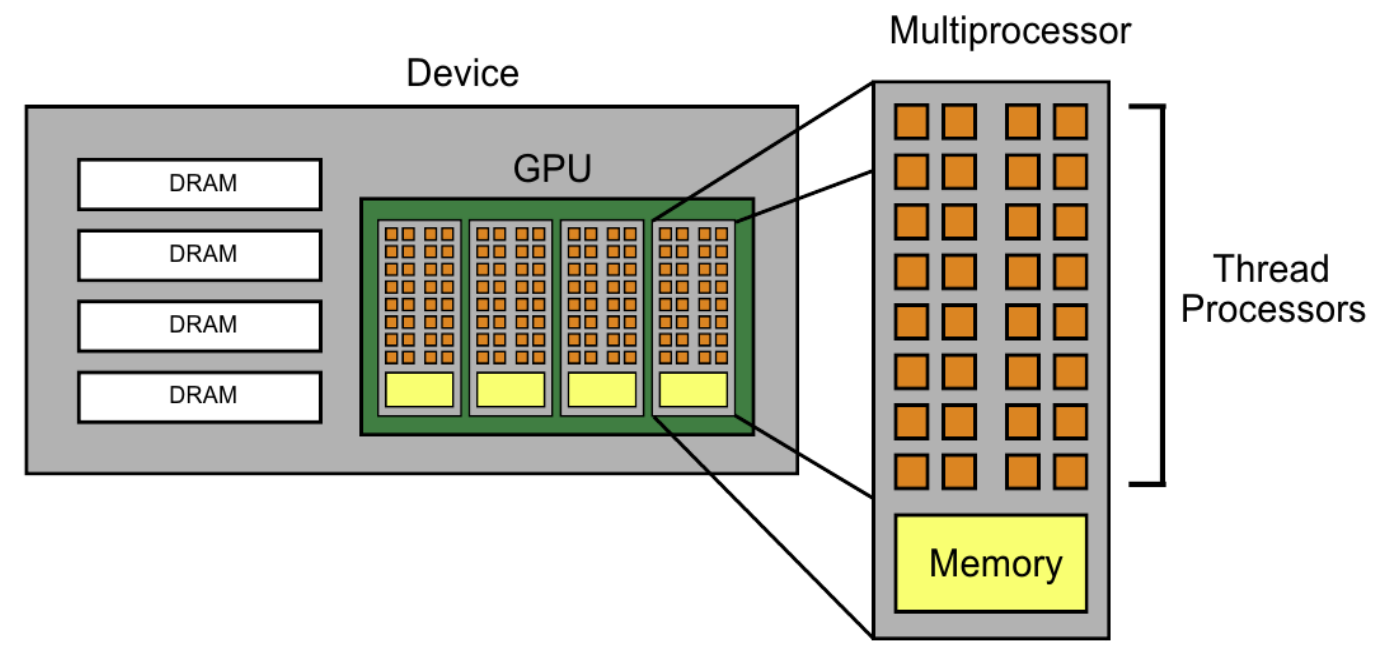

Figure 4.5: Basic architecture of a general purpose graphic computing unit (GPGPU) [119].

The memory hierarchy of the CUDA-Fortran code implementation point of view is defined as follows [120]:

- Global memory: the GPU hardware outermost memory, may be allocated both statically and dynamically in CUDA-Fortan.

- Texture memory: read only memory, has an extremely fast reading when compared with the other memory used for both reading and writing.

- Shared memory: private memory of a single SM, with extremely high reading and writing speeds for use on a single thread block. Its duration is the same of the block.

- Register memory: memory for scalars that are private for a single execution thread. Similar to the shared memory in speed and duration.

The writing of the values through the several memory hierarchies is not always done instantaneously, sometimes writing and reading of wrong values may occur. For an efficient and consistent numerical implementation, the block order execution must be taken into account along with its synchronization in the code. The writing of calculated values into 
the global memory from the shared memory is not done instantaneously. CUDA-Fortran intrinsic synchronization functions [121] like synchthreads and threadfence. The use of these functions guarantees the correct order of writing and reading over the global and shared memories after certain code parts execution.

\subsubsection{CUDA-Fortran Implementation of the VAT Code}

The VAT code has two main kernel subroutines. The most important one, that consumes up to $90 \%$ of the total iteration time, is euler_solver. This kernel performs the numerical calculations required by equations 4.83 to 4.140 . The second one, that consumes up to $5 \%$ of the total iteration time, is immersed_boundary and this kernel performs the numerical calculations required by equations 4.189 to 4.195. Considering other minor kernels, the overall result is that $97 \%$ of the iteration time is spent in the GPU and only $3 \%$ of it is spent in the CPU host. This very small fraction of the total iteration time came from off-loading practically $100 \%$ of the numerical calculations from the CPU host to the GPU device. These execution times were obtained independently by two profiling programs: pgprof (CUDA-Fortran compiling tool) and tau (Tuning and Analysis Utilities) and both programs showed excellent agreement of the results.

In order to off-load the numerical calculations to the GPU, the conservative variables defined by equations 4.126 to 4.130 were allocated by the main program in the GPU global memory using the CUDA Fortran variable qualifier device. Using this approach, the most important variables in the VAT code reside in the GPU global memory and not in the CPU memory, avoiding the communication bottleneck between the GPU and the CPU when the numerical calculations are performed.

With the conservative variables allocated in the GPU global memory, the texture cache of the GPU was used to open a fast and read-only access from the NVIDIA symmetric multiprocessors to the conservative variables using standard Fortran pointers. The last important implementation was to allocate the primitive variables defined by equations 4.122 to 4.125 in the symmetric multiprocessors' shared memory using the variable qualifier shared in the declaration of the primitive variables of the euler_solver kernel.

The CUDA Fortran version of the VAT code was run on a single NVIDIA GeForce GTX 
TITAN GPU, with 896 double-precision cores and 6 gigabytes of RAM memory (CUDA global memory). The time needed for each iteration of the simulated cases of rotor and stator cascades interaction was around 1.1 seconds, where around thirty million control volumes were used in the computational mesh. On the available global memory of the GPU, 32 million control volumes could be simulated on the two dimension version of the code. The operating system that the simulations were carried out was under the Linux distribution openSUSE 12.1 x64.

The computational capacity obtained from a single GPU NVIDIA Titan GTX was equivalent to the CPU parallel version of the VAT code with OpenMP and MPI on 256 CPU cores distributed equally into 8 nodes of the cluster Trestles from the San Diego Supercomputer Center (SDSC). 


\section{Chapter 5}

\section{Numerical Results}

This chapter presents the results of the numerical methodologies described in chapter 4. The first part of this chapter presents the numerical results of a cyclic wave under the effects of expansional viscosity. The theoretical base of results is the linear stability analysis from chapter 3, that will then be compared with the numerical amplification factor.

The second part presents the numerical results from the VAT code, where the numerical validation of the study for the rotor and stator interaction is applied to the subsonic, transonic and supersonic flow regimes. The duct propagation theory and tonal noise characterization are used as the theoretical base of solutions for comparison to the numerical simulations modal response for the subsonic and transonic cases. The shock-wave duct propagation theory and the algorithm of these shock-waves interaction obtained earlier are used for comparison to the numerical results of the supersonic rotor cascade cases.

The third and last part of this chapter is about the synergy of the two lines of research of this work. For the supersonic case, which has high levels of non linearity due to the rotor and stator interaction in supersonic conditions, the expansional viscosity model with instantaneous response is applied to the flow and its effects are evaluated by varying the physical parameter related to the expansional viscosity. 


\subsection{One Dimensional Simulations With the Expansional Viscosity Model}

The numerical simulations of this sections are about the first computational test of the expansional viscosity model applied to the Euler equations in its non linear form with the barotropic model as the closure relation. The numerical methodology described in chapter 4 in used for the discretization process.

The nonlinear form of the governing equations has different behavior compared to the linearized governing equations used in the stability analysis. The proposed problem to be solved numerically is similar the linear stability analysis. A one dimensional wave is the inital condition. Its amplitude decay through time is compared to the decay from the analytical solution of the stability analysis as a validation process for both methods. The physical parameters effects are then assessed upon the expansional viscosity.

For the numerical simulations we will consider the following initial conditions for the velocity, density, in the non dimensional form, and the expansional viscosity on the permanent form:

$$
u(x, t=0)=1+u_{a} e^{i k x}, \quad \rho(x, t=0)=1+\rho_{a} e^{i k x}, \quad \kappa(x, t=0)=\rho\left(r_{M}^{2}-1\right) .
$$

The same numerical value for the oscillation amplitude of the density was used for the oscillation amplitude of the velocity. A cyclic condition is applied at the boundaries of the computational mesh with physical length of $2 \pi / k$ for an infinite wave solution. As as consequence, only one wavelength is numerically solved. The value for the CFL condition is 0.5 , where convergence and stability are guaranteed for all cases. The spectral filter used for the removal of non resolved wave content was applied at every one hundred iterations. The computational mesh resolution is two hundred equally spaced points.

Apart from the linear stability analysis, the amplitudes of the wave variables are now finite in the numerical cases, inducing non linear effects on its behavior. Figure 5.1 shows the case where the expansional viscosity is not considered, where the non linear effects results on the shock formation due to the finite amplitude of the wave. 

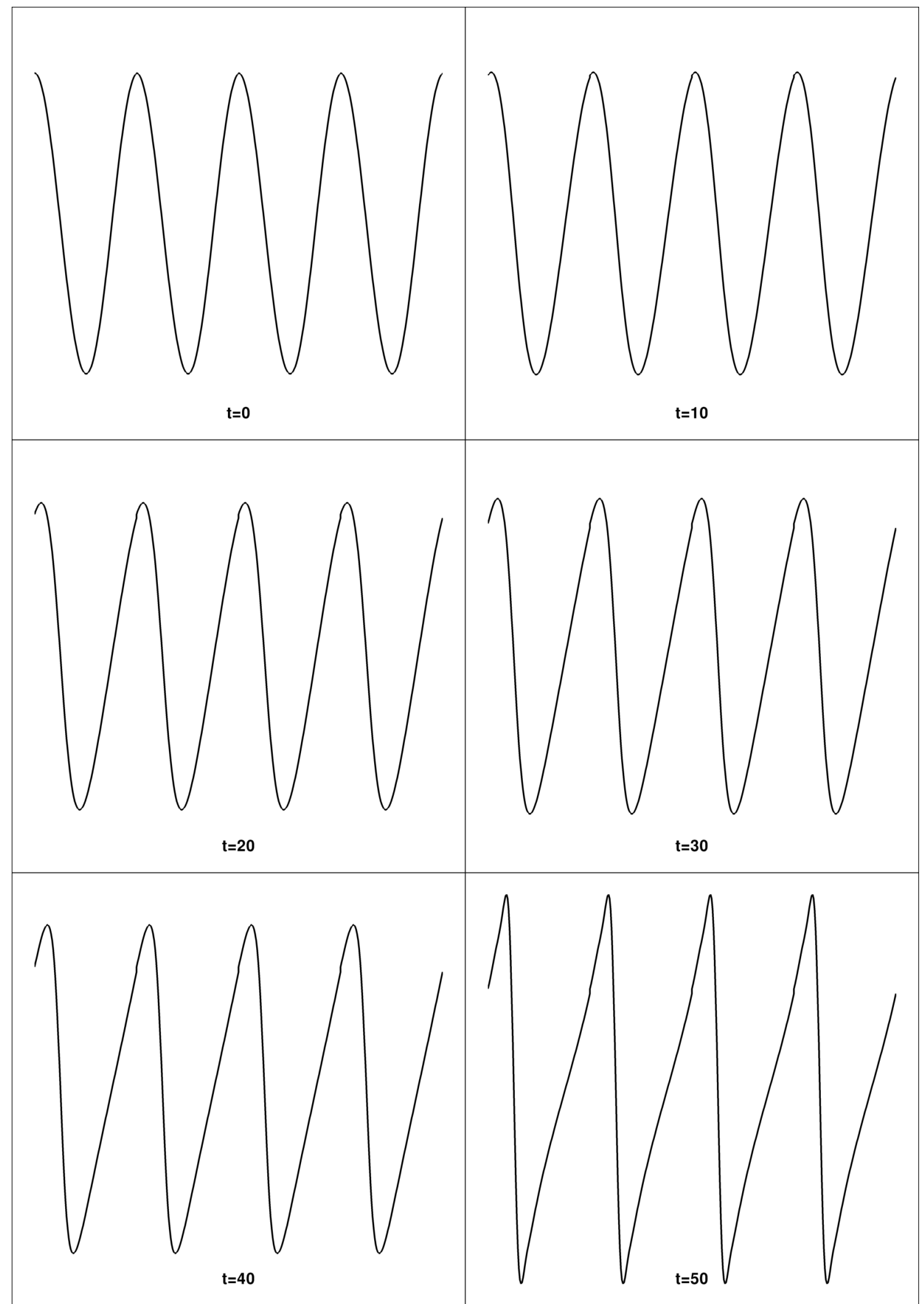

Figure 5.1: Illustrative shockwave formation due to non linear effects directly associated with the wave finite amplitude. The variable shown is the linear momentum $\rho u$. 
For the case depicted in figure 5.1, the positions of the wave have different propagating speeds. Rarefaction and compression points will appear along the wave [20]. The difference in the propagation speeds along the wave induces a change of its shape in time. The compression points move ahead and the rarefaction points retreat. As a result some points along the wave will ultimately tend to non unique values, creating a discontinuity at that point.

The influence of the expansional viscosity is the attenuation of the non linear effects associated with the finite amplitude of the wave. The first test regarding this non linearity is the comparison between the amplification factor obtained from the linear stability analysis and the one from the numerical results. Unlike the condition from the linear stability analysis, the temporal oscillation does not occur naturally in the time domain on the numerical simulations, where a forcing term must be used for this oscillation to occur. By using $\omega=0$ in the stability analysis the same conditions for wave propagation can be obtained for both methods. In the stability analysis, the amplification factor is the solutions of the linear governing equations from chapter 3 , but in the numerical cases the amplification factor must be obtained indirectly from the results. We now consider the following form of the velocity solution in a similar to the stability analysis:

$$
u(x, t)=1+\left(u_{a} e^{\eta t}\right) e^{-i \Omega t+i k x} .
$$

The exponential term outside the parenthesis on equation 5.2 is the oscillatory term with respect to the spatial and temporal point of view in the solution. Now considering the temporal term as equal to zero, only the time decay due to the expansional viscosity will have a time effect on the wave. We also consider that the decay measurement point on the wave, is the wave crest, i.e., the maximum value of speed of the solution as time passes. In this maximum wave value, the term outside the parenthesis in equation 5.2 will be unity, as a consequence the solution at this point will be of the following form:

$$
u(x, t)=1+u_{a} e^{\eta t}
$$

Regarding the numerical solution, from the velocity fluctuation history the wave velocity amplitude $u_{a}$ and the amplification factor $\eta$ may be obtaind and compared with the initial value of $u_{a}$ and the amplification factor obtained from the linear stability analysis. The curve 


\begin{tabular}{c|c}
\hline \hline Theoretical & Numeric \\
\hline 0,00001 & 0,00001 \\
0,00005 & 0,00005 \\
0,0001 & 0,0001 \\
0,0005 & 0,0005 \\
0,001 & 0,0009999 \\
0,005 & 0,004998 \\
0,01 & 0,009993 \\
0,05 & $0,0,04983$ \\
0,1 & 0,9934 \\
0,2 & 0,1975 \\
0,3 & 0,2948 \\
0,4 & 0,3912 \\
0,5 & 0,4869 \\
\hline \hline
\end{tabular}

Table 5.1: Theoretical and numerical comparison of the initial wave amplitude $u_{a}$.

fitting was obtained with the least squares method [124]. The flow conditions considered for this validation case were of $r_{M}=1,05, \Psi=1,0, \beta=1,0, \gamma=1,4, R e_{\kappa}=0,5$ e $k=2$. The simulation physical time interval was of $t=1$, enough to obtain an asymptotic condition at the end of the simulated physical time. The theoretical values for the amplification factor were of $\eta_{1}=-0.41$ and $\eta_{2}=-0.41$, resulting in an effective solution of $\eta=-0.82$ by the linear combination of the two solutions to be compared with the numerical results.

Several values of velocity and density for the initial wave amplitudes were tested and the decay results compared with the linear stability analysis. Table 5.1 shows that the regression values related to the initial wave amplitude agree really well with the actual initial values for the numerical method. Figure 5.2 shows the amplification factor obtained from the linear stability analysis, which is independent of the initial wave amplitude, and the obtained values from the numerical simulations. An excellent agreement between both methods can be observed for wave amplitude values smaller than $10^{-3}$. For larger values of the wave amplitude, the damping effect of the expansional viscosity become smaller due to the afore mentioned non linear effects of momentum accumulation on the wave crest and 


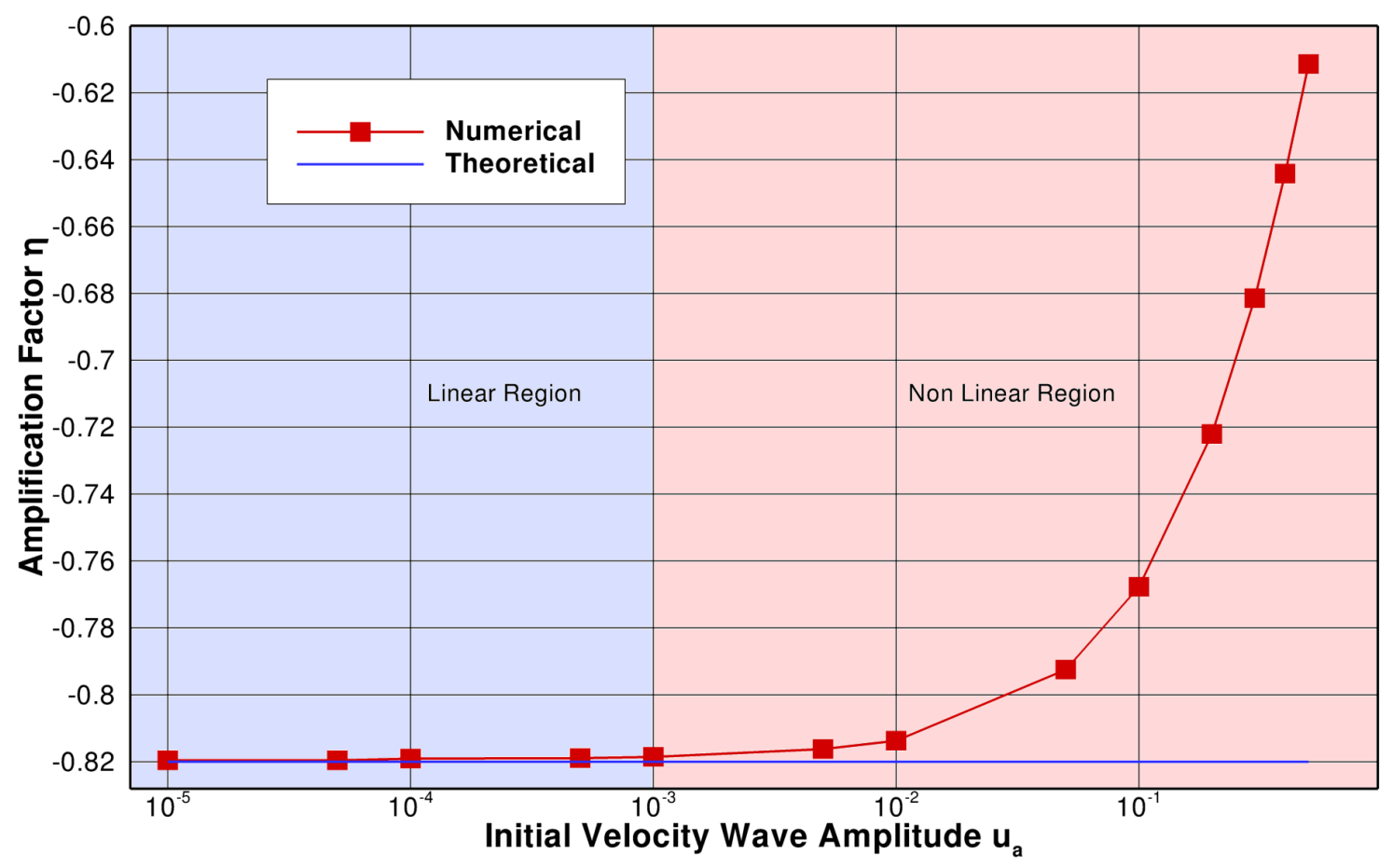

Figure 5.2: Linearity level of the theoretical and numerical amplification factor comparison.

trough as seen in figure 5.1. This momentum accumulation, non existent in the linear cases, reduces the wave decay rate due to the expansional viscosity.

Regarding the physical parameters sensivity analysis, the most important ones related to the expansional viscosity are assessed: the spatial wavenumber $k$, the relaxation time non dimensional parameter $\Psi$, the expansional Reynolds number $R e_{\kappa}$ and the ratio of propagation velocities $r_{M}$. The initial value for the wave amplitudes for velocity and density are $u_{a}=\rho_{a}=10^{-2}$. Figure 5.3 shows the wave velocity amplitude decay sensivity for three order of magnitude for the wavenumber $k$. Identical curves for the time decay for the three cases can be observed. As expected, the expansional viscosity acts in a more intense way for larger values of wavenumber, i.e., waves with higher frequency. For the values shown in figure 5.3, an order of magnitude higher for the wavenumber results in a reduction of two orders of magnitude on the wave amplitude time decay.

The next physical parameter to be assessed is $\Psi$, directly related to the flow characteristic time and the fluid non equilibrium relaxation time, as shown in figure 5.4. The amplitude of velocity does not show any effect due to the $\Psi$ parameter, in all three cases 


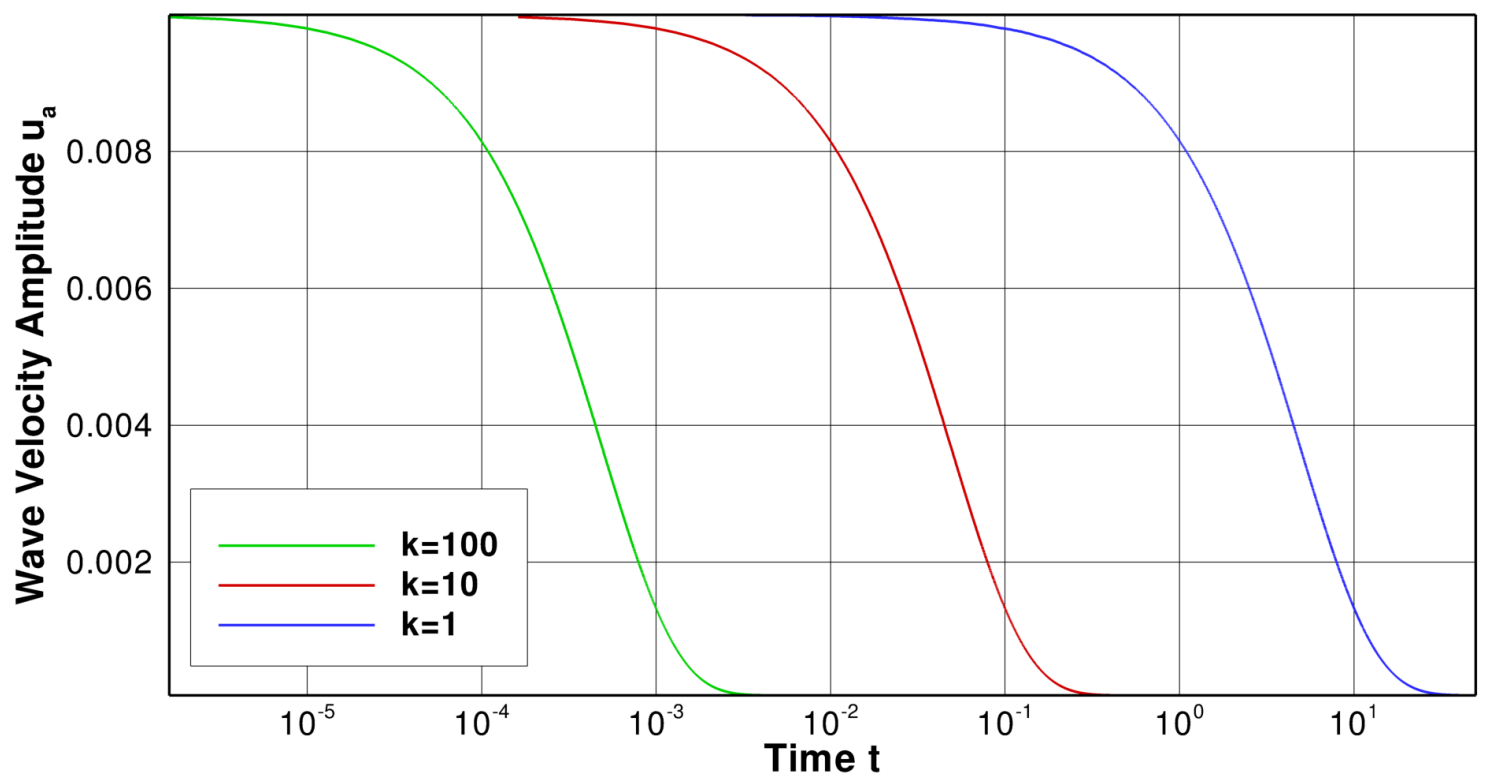

Figure 5.3: Wavenumber $k$ sensivity for three order of magnitude with the following parameters: $R e_{\kappa}=0,5, \Psi=1,0, \beta=1,0, \gamma=1,4$ e $r_{M}=1,05$.

the decay of the velocity amplitude remains the same. For the fluid density a slight effect of the $\Psi$ parameter becomes apparent, even though its effect is minimal, the difference between the curves can be noted in figure 5.4. The most interesting effect of the $\Psi$ parameter is the time evolution of the modulus of the expansional viscosity at the wave crest. As $\Psi$ is proportional with the flow characteristic time and inversely proportional to the fluid relaxation time, the expansional viscosity response observed in the third graph from figure 5.4 shows the behavior of these characteristic times. A large value for $\Psi$ shows a fast response of the expansional viscosity to obtain an asymptotic value. A small value of the $\Psi$ parameter shows a damped oscillation of the expansional viscosity through time until a final value is obtained.

Figures 5.5 and 5.6 show the sensivities of the expansional Reynolds number and the ratio of propagation speeds in and out of the thermodynamic equilibrium, respectively. The sensivity obtained for these physical parameters on the velocity, density and expansional viscosity wave amplitudes follows a fixed trend at their propostions. With the rise of the expansional Reynolds number $R e_{\kappa}$, the density and expasional viscosity values rises, while at the wave crest the velocity gets smaller through time. The opposite effect was 
obtained for the ratio of propagation speeds $r_{M}$, as $r_{M}$ increases the crest wave value for the density and expansional viscosity modulus get smaller and larger values are obtained for the velocity.
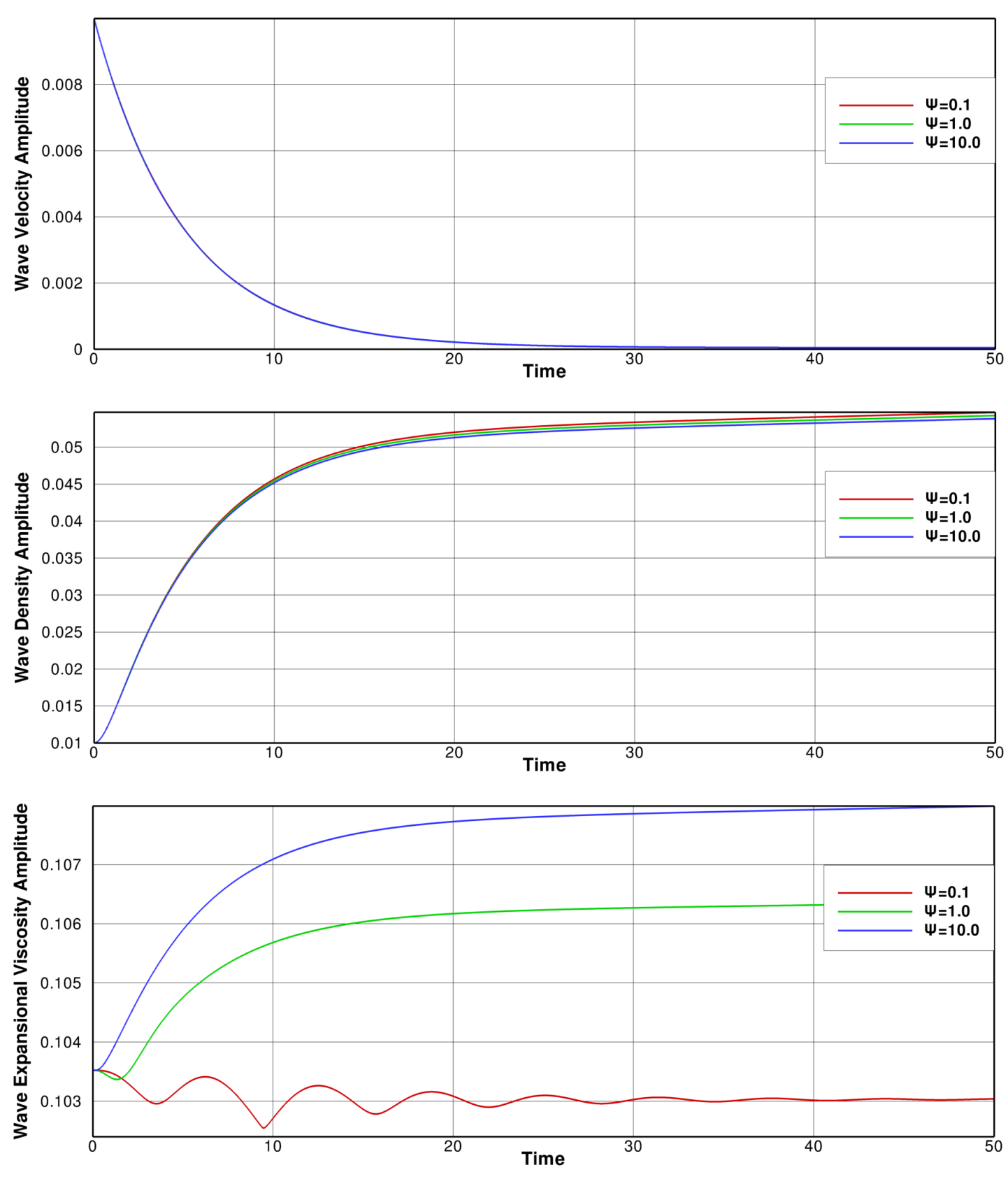

Figure 5.4: Ratio of flow and fluid relaxation characteristic times $\Psi$ sensivity with the following parameters: $R e_{\kappa}=0,5, k=1,0, \beta=1,0, \gamma=1,4$ e $r_{M}=1,05$. 

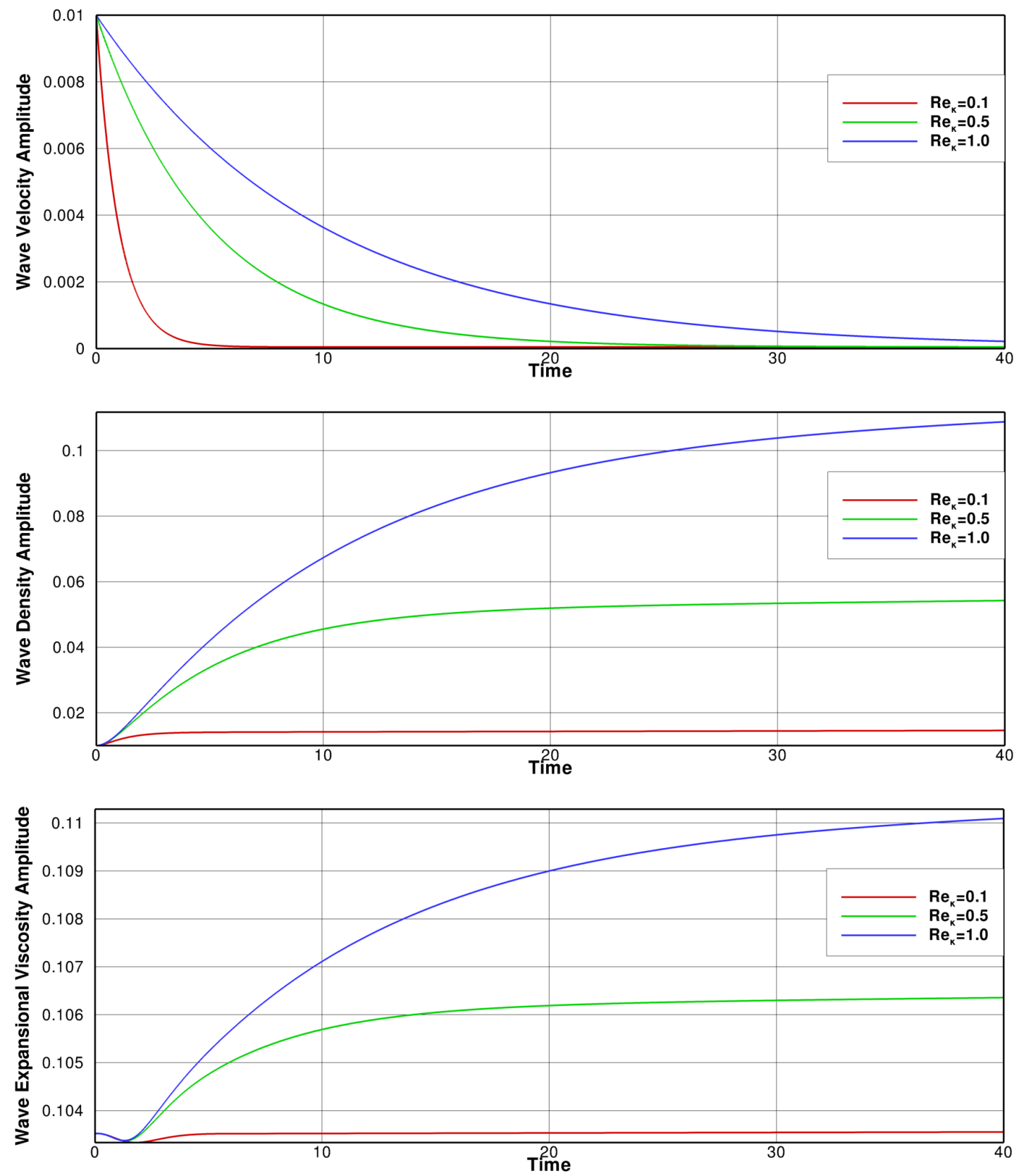

Figure 5.5: Expansional Reynolds number $R e_{\kappa}$ sensivity with the following parameters: $\Psi=1,0, k=1,0, \beta=1,0, \gamma=1,4$ e $r_{M}=1,05$. 

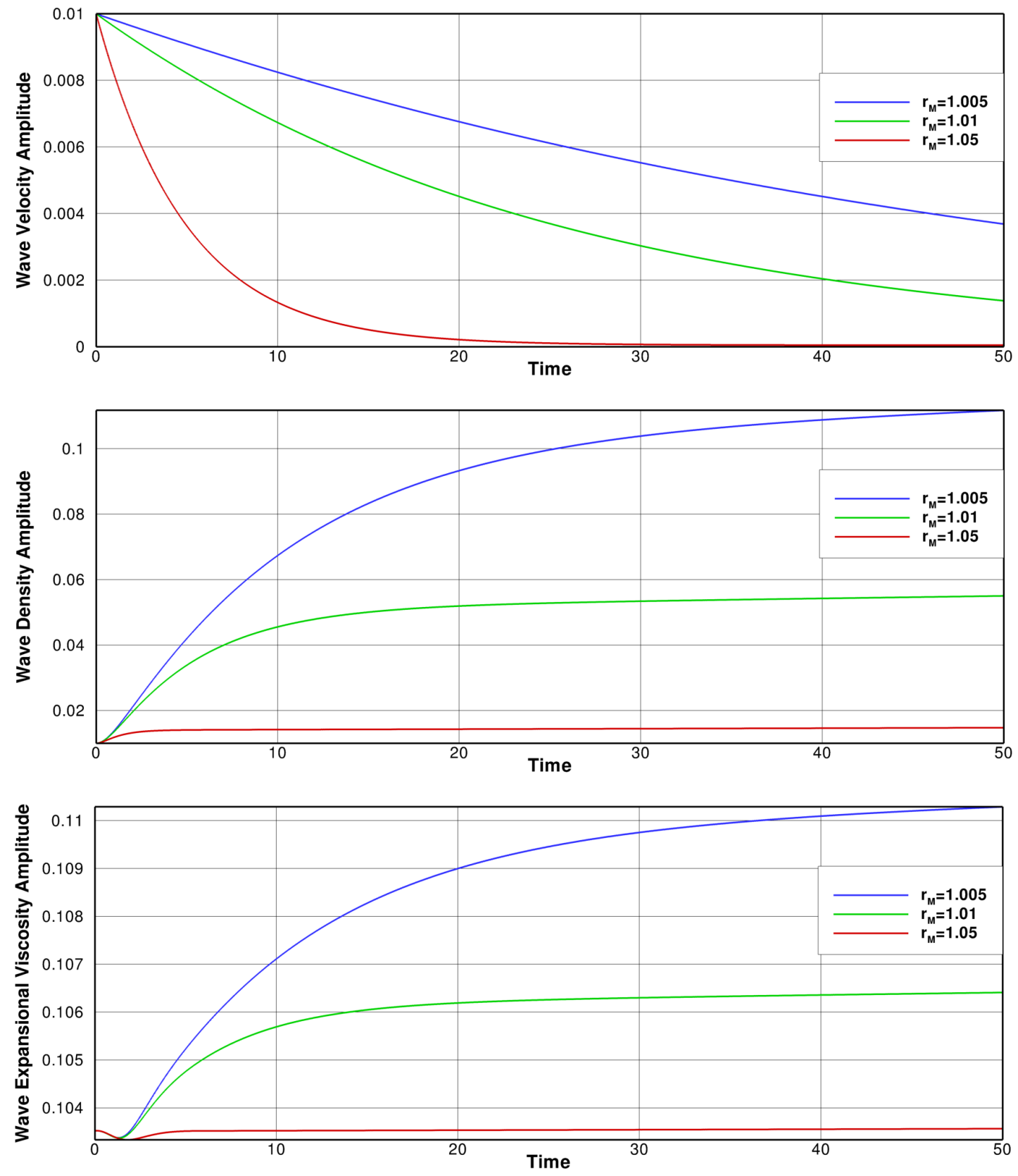

Figure 5.6: Ratio of propagation speeds in and out of the thermodynamic equilibrium $r_{M}$ sensivity with the following parameters: $\Psi=1,0, k=1,0, \beta=1,0, \gamma=1,4$ e $\operatorname{Re}_{\kappa}=0,5$. 


\subsection{Rotor and Stator Cascades Interaction Numerical Simulatons}

\subsubsection{Rotor and Stator Cascades Simulations in Subsonic Regime}

The blade and vane geometries used in the numerical simulations, shown in figure 5.7, corresponds to the profile sections at the tip of the rotor blades and stator vanes of the Advanced Noise Control Fan (ANCF), a fan rig from the Aeroacoustics Branch of the NASA Glenn Research Center.
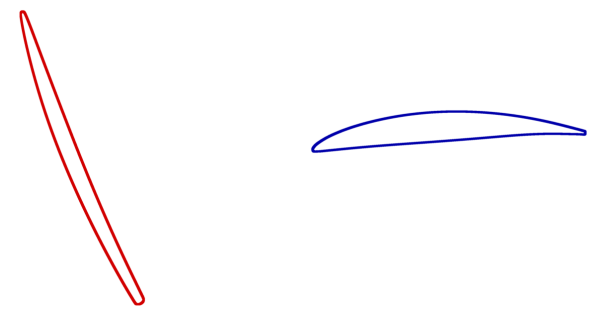

Figure 5.7: Rotor blade (red) and stator vane (blue) geometries.

The extent of the computational domain with a regular Cartesian mesh is presented in figure 5.8, showing a rotor cascade with 16 blades (red) and a stator cascade with 13 vanes (blue), as well as the position of the far-field (black point) and near-field (green point) probes, the horizontal inflow velocity $U_{\infty}$ (blue arrow) and the vertical blade velocity $U_{B}$ (red arrow).

The characteristic length of the setup is the pitch of the blades cascade, $L_{p_{B}}(0.2394 \mathrm{~m})$, so the dimension of the computational domain in the vertical direction is $16 L_{p_{B}}(3.8302$ $\mathrm{m})$ and the dimension in the horizontal direction is $13 L_{p_{B}}(3.1121 \mathrm{~m})$. Since the Cartesian grid has a resolution of 300 control volumes along the pitch of the blades cascade, the total number of control volumes in the regular computational domain is 18,720,000. Adding the control volumes in the stretched regions upstream and downstream of the regular region, the total number of control volumes in the domain is 20,160,000. Figure 5.9 gives an idea of the resolution levels of the Cartesian and surface grids at the leading edge of the blade.

In order to avoid numerical oscillations and wave reflextions, there are 154 control 


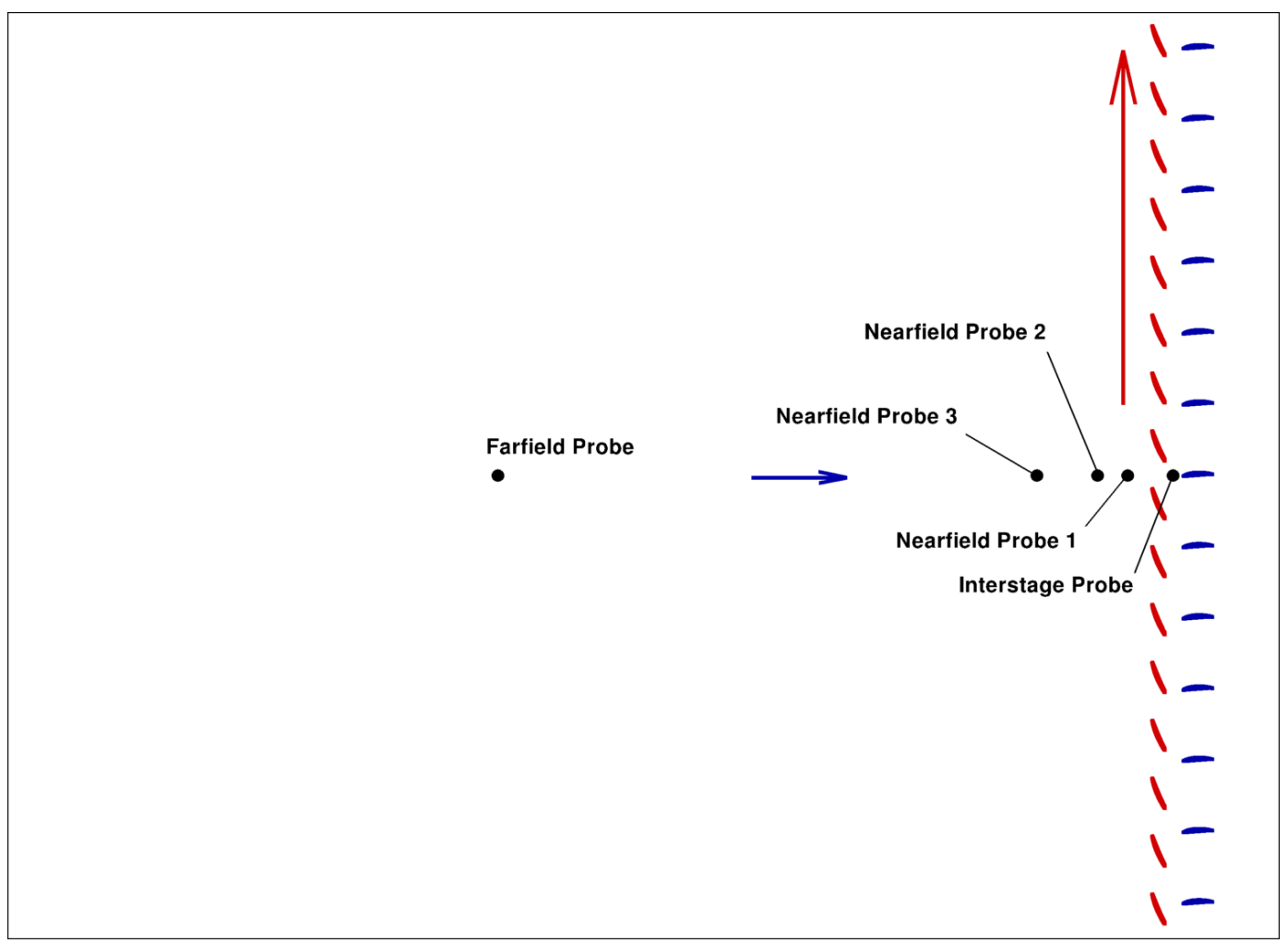

Figure 5.8: Computational domain of the regular cartesian mesh.

volumes on the inlet and outlet mesh boundaries. The first control volume on the non regular region has the same horizontal length as its neighbor in the regular region. The length of the second control volume is 1.05 times of its neighbor closer to the regular region and so on until the last control volume of the non regular part of the computational mesh. The horizontal length of the last control volumes is so larger than the length of the control volumes of the regular region, that any acoustic wave or any other traveling gradient are damped by the lack of resultion of the non-regular mesh and any disturbance will not reflect back to the regular region because the physical time needed is larger than the simulated physical time.

At the left and right ends of the computational mesh, the values from conservative variables vector $\mathbf{U}$ of the mesh edge control volumes are repeated on two ghost control volumes neighboring these control volumes. Due to the large volume of these control volumes when compared to the regular mesh region control volumes, the prescribed reference values are maintained through all the simulation time. At the upper and lower control volumes, a cyclic boundary condition is applied. By using two ghost control volumes at the lower and 
upper edges of the computational mesh, the values of the conservative variables vector $\mathbf{U}$ are repeated from their opposite side counterparts to ensure the cyclic condition.

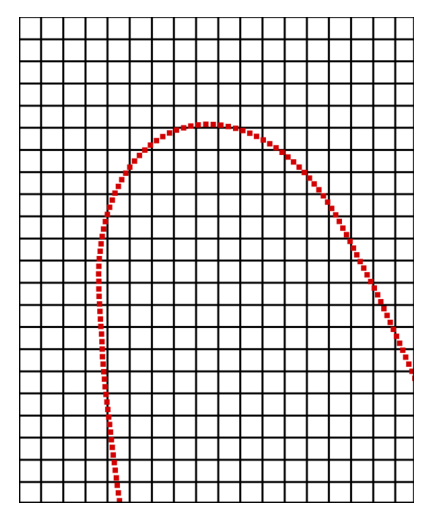

Figure 5.9: Grid resolution at the leading edge of the blade.

The flow conditions used for all cases are very close to the operational conditions of the ANCF rig. The horizontal inflow velocity is $U_{\infty}=41.39 \mathrm{~m} / \mathrm{s}$, the inflow pressure and temperature are $p_{\infty}=101,300 \mathrm{~Pa}$ and $T_{\infty}=300 \mathrm{~K}$, respectively, resulting in an inflow Mach number $M_{\infty}=0.12$. For a shaft angular velocity of $30 \mathrm{rps}$ and a tip radius of $24 \mathrm{in}$, the equivalent vertical velocity of the blade cascade is $U_{B}=114.9 \mathrm{~m} / \mathrm{s}$ with $M_{B}=0.33$, measured from an inertial frame of reference that is fixed to the stator vanes.

The numerical time step $\Delta t$ is given by [23]::

$$
\mathrm{CFL}=\frac{U_{\infty}+c_{\infty}}{\left(\frac{\Delta x}{\Delta t}\right)} .
$$

In the cascade simulation cases of this work, the value for the CFL condition is approximately 0.75 . In all cases enough iterations were run to obtain a complete developed flow, i.e., the perturbations reached all the regular region of the computational mesh and the modal responses are already cyclic. After this state is reached, at least one million of iterations are run for the pressure probes measurement.

In flow visualization figures, the visualization variable $\beta_{T}$ is used. It is based on the magnitude of the temperature gradient as [108]:

$$
\beta_{T}=|\nabla T|^{1 / 10}
$$

This variable is used since it can simultaneously show acoustics waves, that are characteristic of acoustic fields, and vorticity regions, that are characteristic of aerodynamic fields. 
For the Fourier transform of the pressure probes time history, the presented scale of the signal strenght is the root mean square (RMS). Defined as:

$$
p_{r m s}^{\prime}=\sqrt{\frac{1}{t_{2}-t_{1}} \int_{t_{1}}^{t_{2}}\left[p^{\prime}(t)\right]^{2} d t}
$$

where the RMS calculation is done over the fluctuation of the pressure signal $p^{\prime}(t)$ in the integrating time interval $t_{2}-t_{1}$.

\subsubsection{Subsonic Rotor Only}

The rotor alone is a very good test of the moving-body high-order immersed boundary method, since all the cascade blades are moving with the vertical velocity $U_{B}=114.9 \mathrm{~m} / \mathrm{s}$, as shown in figure 5.10. From the acoustics point of view, using the Tyler-Sofrin selection rule (equation 2.126) for the rotor cascade $(V=0)$ yields $m=h B=16 h$, i.e., all the generated modes are hamonics of the number of blades in the cascade.

Solving equation 2.130 for the stator cascade yields $\sigma_{m 1, c}=5.32 h$ and since for cascades $\sigma_{m 1}=m$ (equation 2.142), all the modes generated by the rotor are cut-off since

$$
\sigma_{m 1, c}=5,32 h<16 h=m=\sigma_{m 1}, \quad \text { for } \quad h=1,2,3,4, \ldots \ldots
$$

The spectra of the root mean square (RMS) value of the pressure fluctuation is shown in figure 5.11, where the green curve corresponds to the spectrum for the near-field static probe and the black curve to the spectrum for the far-field static probe. In this figure is possible to see, for the near-field static probe, a very strong tonal peak for the first harmonic of the blade-passing frequency, generated by the moving blades associated to the mode $m=16$ but no sign of propagation is observed into the far-field probe. These results show that the moving-body immersed boundary methodology is capable of generating the correct rotor modes, but they are cut-off as predicted by the linear propagation theory, showing that as in the previous case, no spurious modes are generated and/or propagated.

Figure 5.10 also shows a vorticity wake for each rotor blade. These wakes are not spurious, since they are generated by the pressure-gradient source term of the two-dimensional vorticity equation (equation IV.10 from appendix IV). It is worth noting that there are no signs of vorticity generation in the blades surfaces, since the boundary conditions for the 


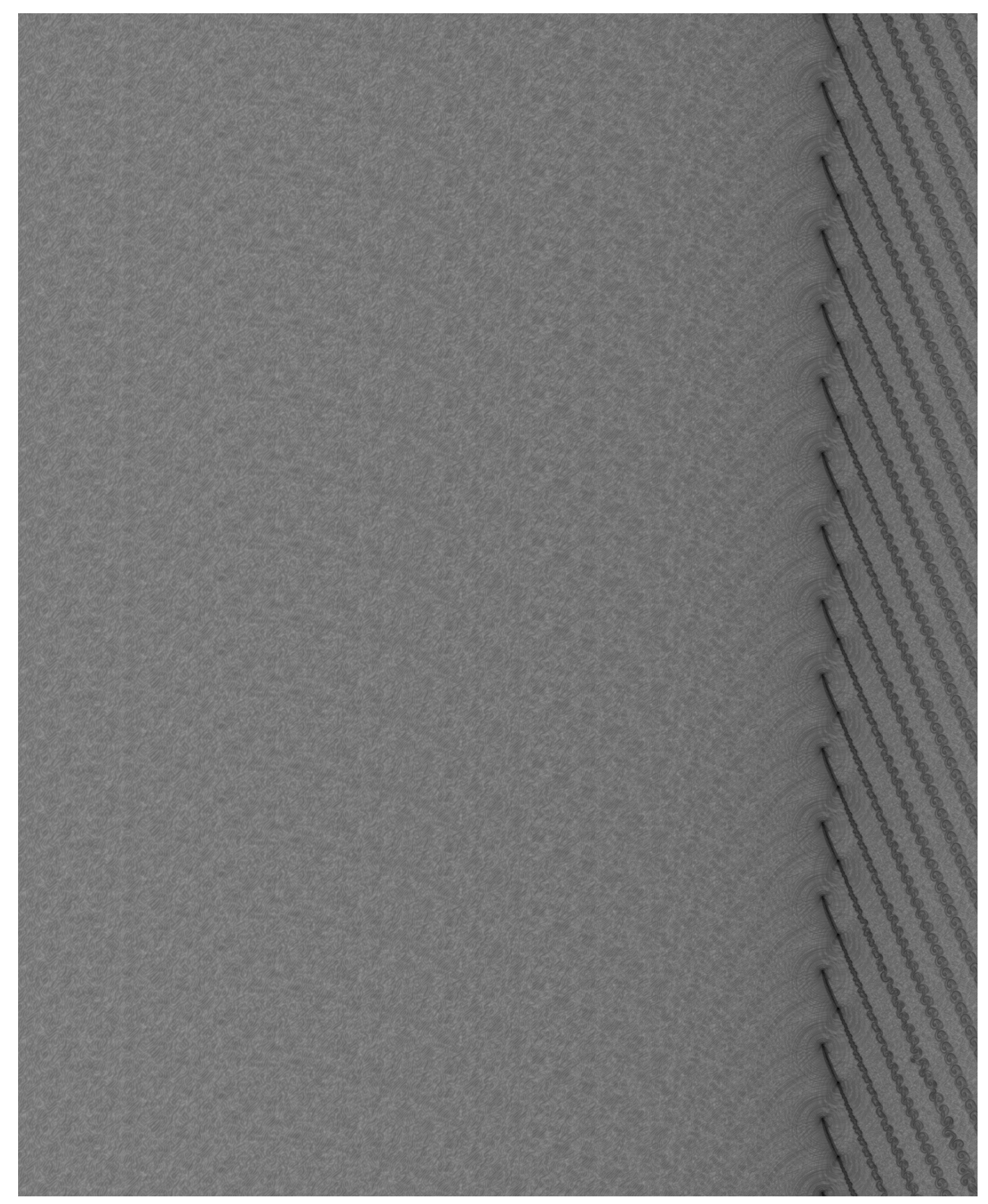

Figure 5.10: Visualization of the instantaneous aeroacoustic field generated by the rotor cascade.

Euler equation specify that $(\nabla p)_{n}$ and $(\nabla \rho)_{n}$ are both zero in the normal direction of the surface, so the only non-zero gradients are those in tangencial direction, $(\nabla p)_{t}$ and $(\nabla \rho)_{t}$ and since they are aligned, the pressure-source term in the vorticity equation is zero over the blades surface. 


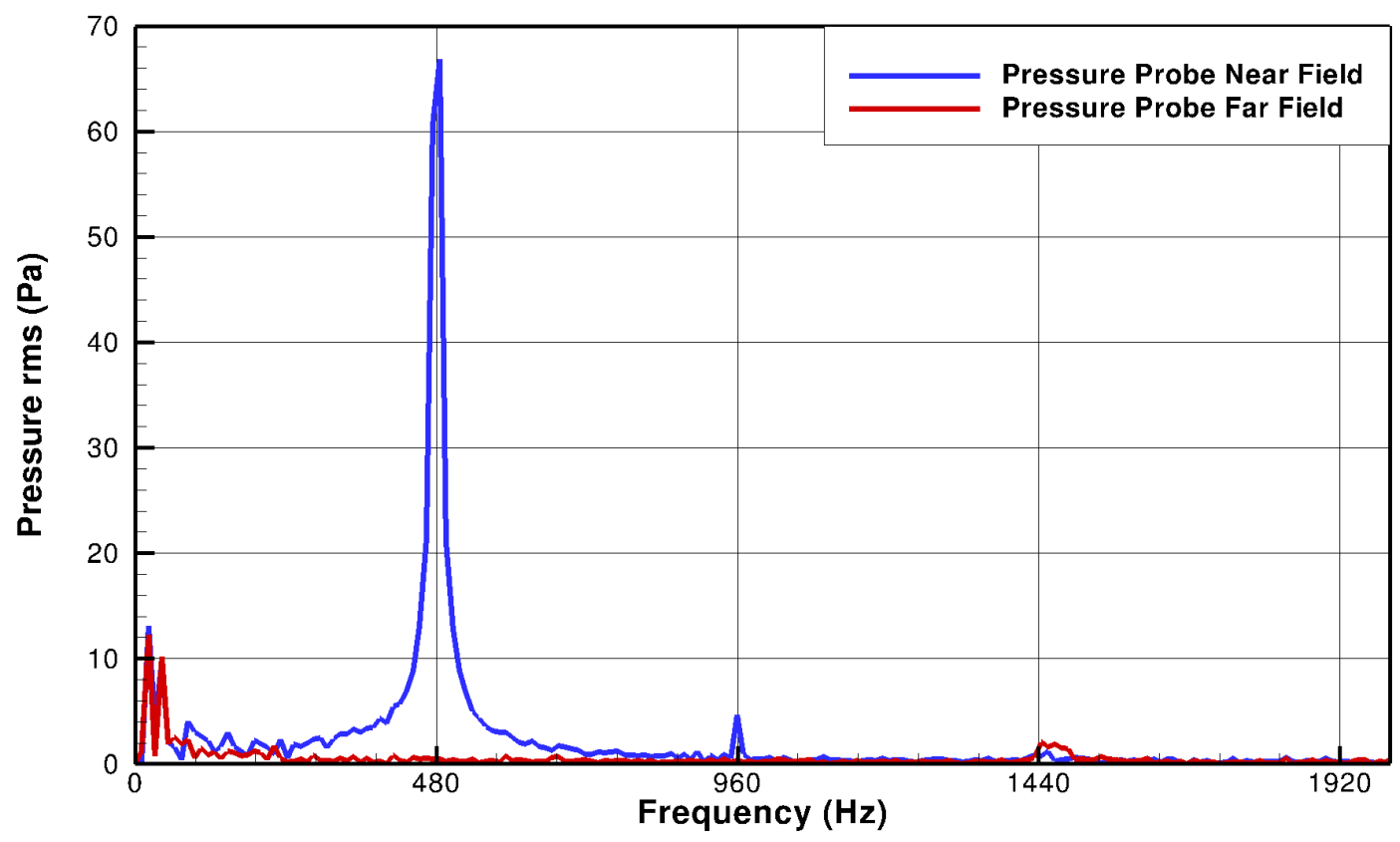

Figure 5.11: Spectra of the RMS value of the pressure fluctuation for the near-field (blue) and far-field (red) static probes.

\subsubsection{Subsonic Rotor and Stator Interaction}

Unlike the previous case where all the generated modes are cut-off, in this case there are cut-on modes, as shown by the numerical results presented in figure 5.12 and by the theoretical results present in table 5.2. In this table the critical values of $\sigma_{m 1}$ are calculated up to the fourth harmonic using equation 2.130. The cut-on modes are defined by initially using the Tyler-Sofrin selection rule, with $B=16$ and $V=13$, to assess which modes are generated. Then using the thin annular duct approximation for cascades $\left(\sigma_{m 1}=m\right)$, the cut-on modes are defined as the ones that comply with $|m|<\sigma_{m 1, c}$. Table 5.2 also shows the modal wave-front angles, $\theta$.

It is important to note that the relation $(\mathrm{m} / \mathrm{h})$ in equation 2.141 defines families of cut-on modes that have the same wave-front angle. From the above table it is possible to see that $\theta=59.44^{\circ}$ for the following mode/harmonic relations:

$$
\frac{m}{h}=\frac{3}{1}=\frac{6}{2}=\frac{9}{3}=\frac{12}{4}=3.0
$$




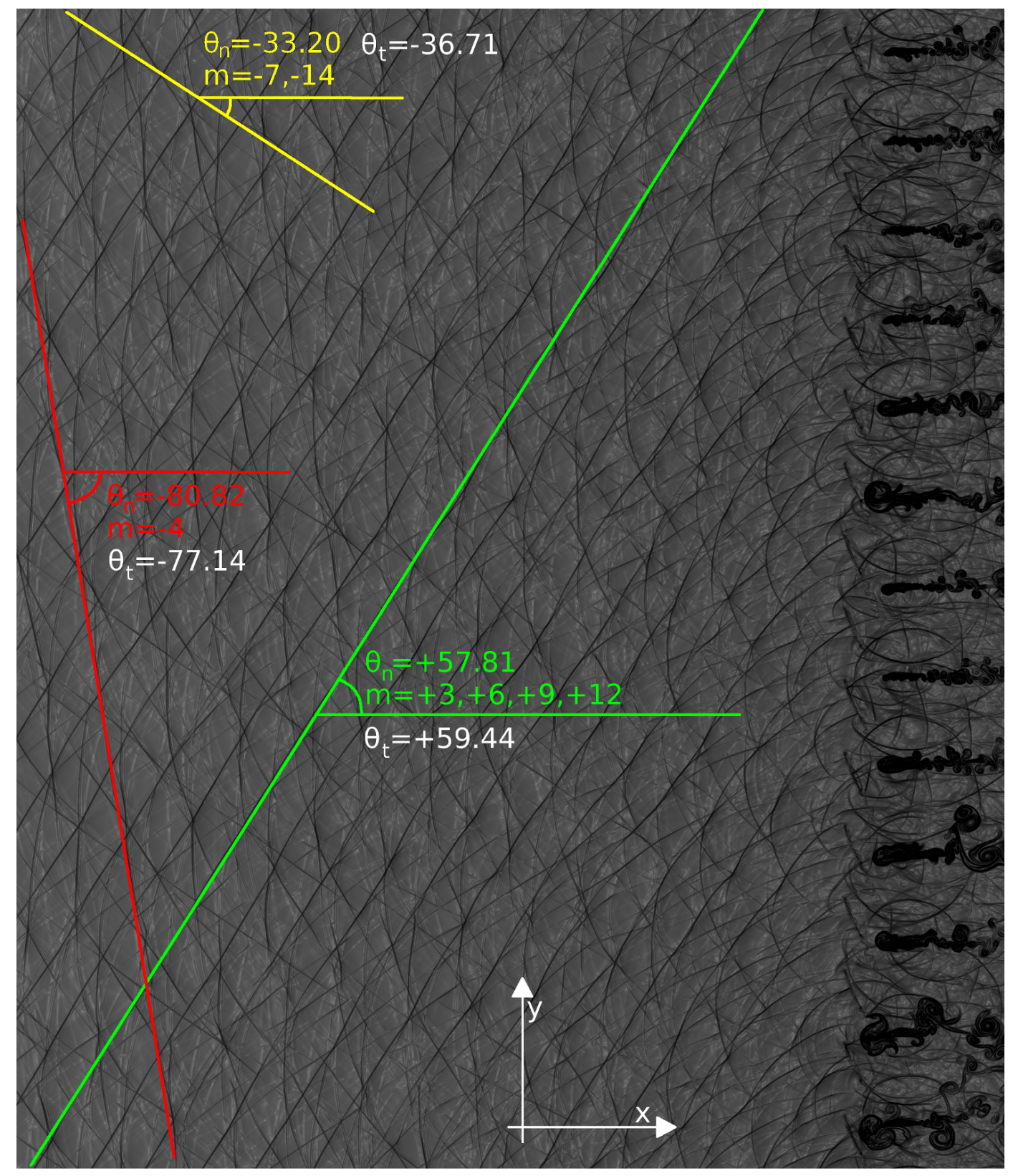

Figure 5.12: Visualization of the instantaneous aeroacoustic field $\beta_{T}$ generated by the interaction of the rotor and stator cascades.

This family is represented in Fig. 5.12 by the green line that was manually drawn parallel to the numerical wave-fronts associated with the above modes. The measured numerical wave-front angle is $\theta_{n}=57.81^{\circ}$ and this value compares very well with the theoretical value given by table 5.2 .

Another family is associated with $\theta=-36.71^{\circ}$, where the harmonic/mode relation is 


\begin{tabular}{c|cccc}
\hline \hline$h$ & 1 & 2 & 3 & 4 \\
\hline$f_{b p, h}$ & $480 \mathrm{~Hz}$ & $960 \mathrm{~Hz}$ & $1440 \mathrm{~Hz}$ & $1920 \mathrm{~Hz}$ \\
\hline$\sigma_{m 1, c}$ & 5,32 & 10,65 & 15,98 & 21,31 \\
\hline$m$ & +3 & $-7,+6$ & $-4,+9$ & $-14,-1,+12$ \\
\hline$\theta$ & $59.44^{\circ}$ & $-36.71^{\circ}, 59.44^{\circ}$ & $-77.14^{\circ}, 59.44^{\circ}$ & $-36.71^{\circ},-82.39^{\circ}, 59.44^{\circ}$ \\
\hline \hline
\end{tabular}

Table 5.2: Theoretical cut-on modes and and wave-front angles from the first to the fourth harmonic.

given by:

$$
\frac{m}{h}=\frac{-7}{2}=\frac{-14}{4}=-3.5 .
$$

This family is represented in figure 5.12 by the blue line drawn parallel to the numerical wave-fronts. The measured numerical wave-front angle is $\theta_{n}=-33.20^{\circ}$ and this value also compares well with the theoretical value. It is important to note that the previous family, $m / h=3.0$, is composed by 4 members and the family $m / h=-3.5$ is composed for only 2 members, so it is natural that the former is more well defined than the later in figure 5.12.

The last visible family in figure 5.12 has only one member and it is associated with $\theta=-77.14^{\circ}$, with a harmonic/mode relation:

$$
\frac{m}{h}=\frac{-4}{3}=-1.3333 \text {. }
$$

This family is represented in Fig. 5.12 by the red line. The measured numerical wave-front angle is $\theta_{n}=-80.82^{\circ}$ and this value compares well with the theoretical value.

Another alternative to identify the numerical cut-on modes is to perform a mode decomposition using the Doppler effect by taking pressure over time measurements with a far-field probe that is moving at a selected vertical speed, $U_{m p}$, that is usually a fraction of the rotor cascade speed, $U_{B}$. Using this approach, the modal frequency shift around a given harmonic is given by Eq. 2.145 .

Figure 5.13 show the spectra of the RMS value of the pressure fluctuation for twodifferent far-field probes: a static (black spectrum) and a moving (blue spectrum) probe. The later is moving with a vertical velocity $U_{m p}=57.45 \mathrm{~m} / \mathrm{s}$, yielding $\delta=U_{B} / U_{m p}=0.5$. In this figure all the spectrum peaks associated with the cut-on modes presented in table 5.2 are identified by the shift in their frequency and, also, all the peaks associated to 
the harmonics are identified. Although the harmonic frequencies slightly differ from the theoretical ones, there is a very good agreement in the modal frequency shift around the first, second and fourth harmonics, and even though the third harmonic is not centered around a single frequency, the tonal shift of its modes is approximately correct.

The numerical results present in figure 5.13 compares very well with the theoretical predictions presented in table 5.2 for the cut-on modes and associated harmonics, showing that the moving-body immersed boundary methodology used in this work is capable of predicting the generation and propagation of acoustics modes due to the subsonic rotorstator interaction in two-dimensional cascades.

From an aerodynamic point of view, it is worth noting that the massive vorticity region around the stator is due to the pressure source term in the vorticity equation. This strong source is associated to the actual movement of the rotor cascade in the computational domain, generating a very strong and unsteady aerodynamic loading over the stator cascade.

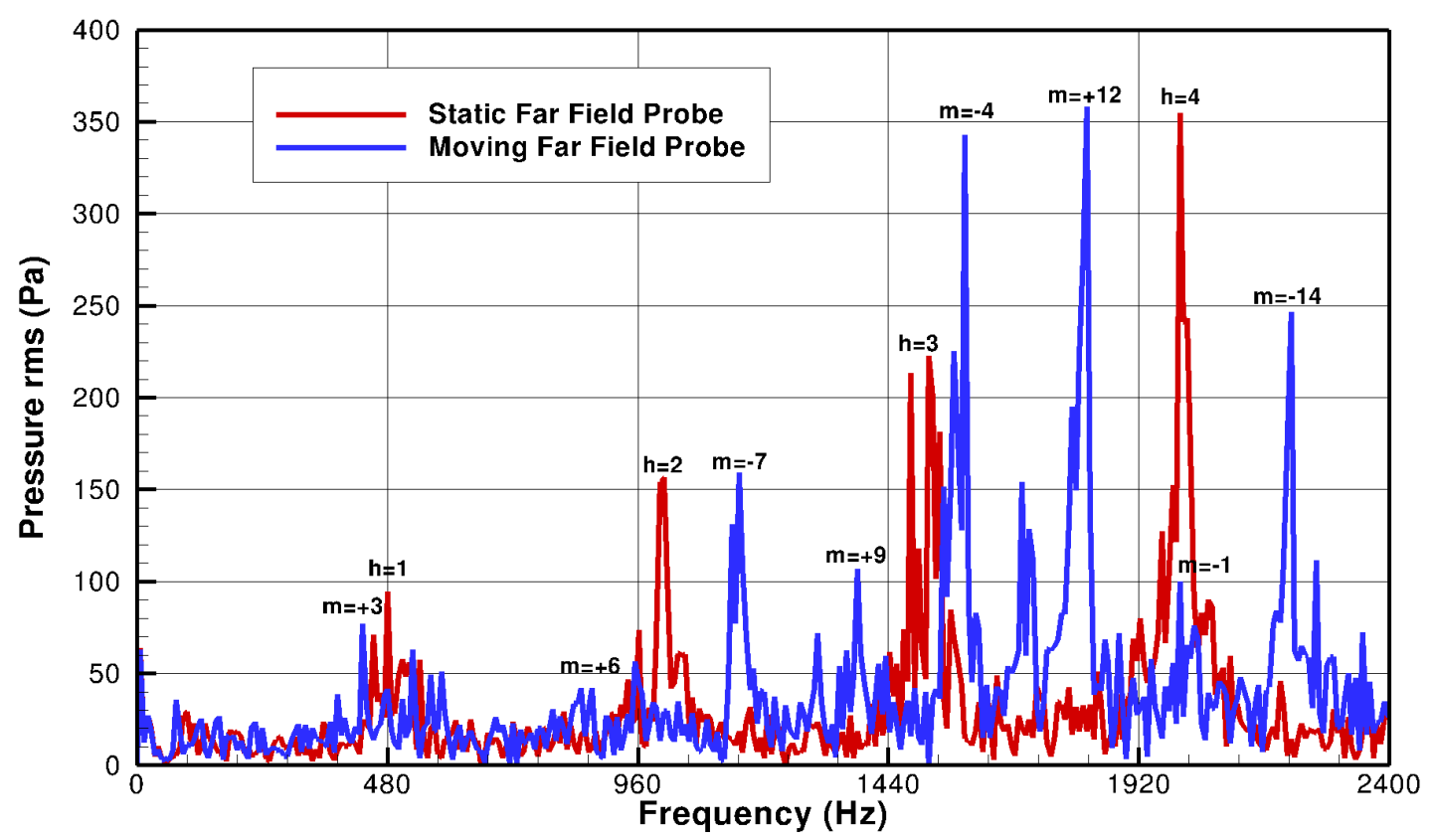

Figure 5.13: Spectra of the RMS value of the pressure fluctuations for a static far-field probe (red) and a moving far-field probe (blue) showing the mode decomposition around each harmonic. 


\subsubsection{Rotor and Stator Cascades Simulations in Transonic Regime}

The rotor blades and stator vanes geometries used in the transonic cascade interaction case is the same used in the subsonic cascade interaction case, following the same proportion for the velocities triangle and keeping geometric similarity. The inlet pressure of the flow is $p_{\infty}=101,300 \mathrm{~Pa}$ and the inlet temperature is $T_{\infty}=300 \mathrm{~K}$. The rotor blades vertical velocity is $U_{B}=312.3 \mathrm{~m} / \mathrm{s}$ with a Mach number of $M_{B}=0.9$, the inlet undisturbed horizontal velocity is $U_{\infty}=112.88 \mathrm{~m} / \mathrm{s}$ with a relative Mach number of $M_{\infty}=0.32$. The corresponding shaft rotation frequency is 81.5 complete turns per second on the equivalent radius of 24 inches $(0.6096 \mathrm{~m})$. The characteristic length is the same as the subsonic case.

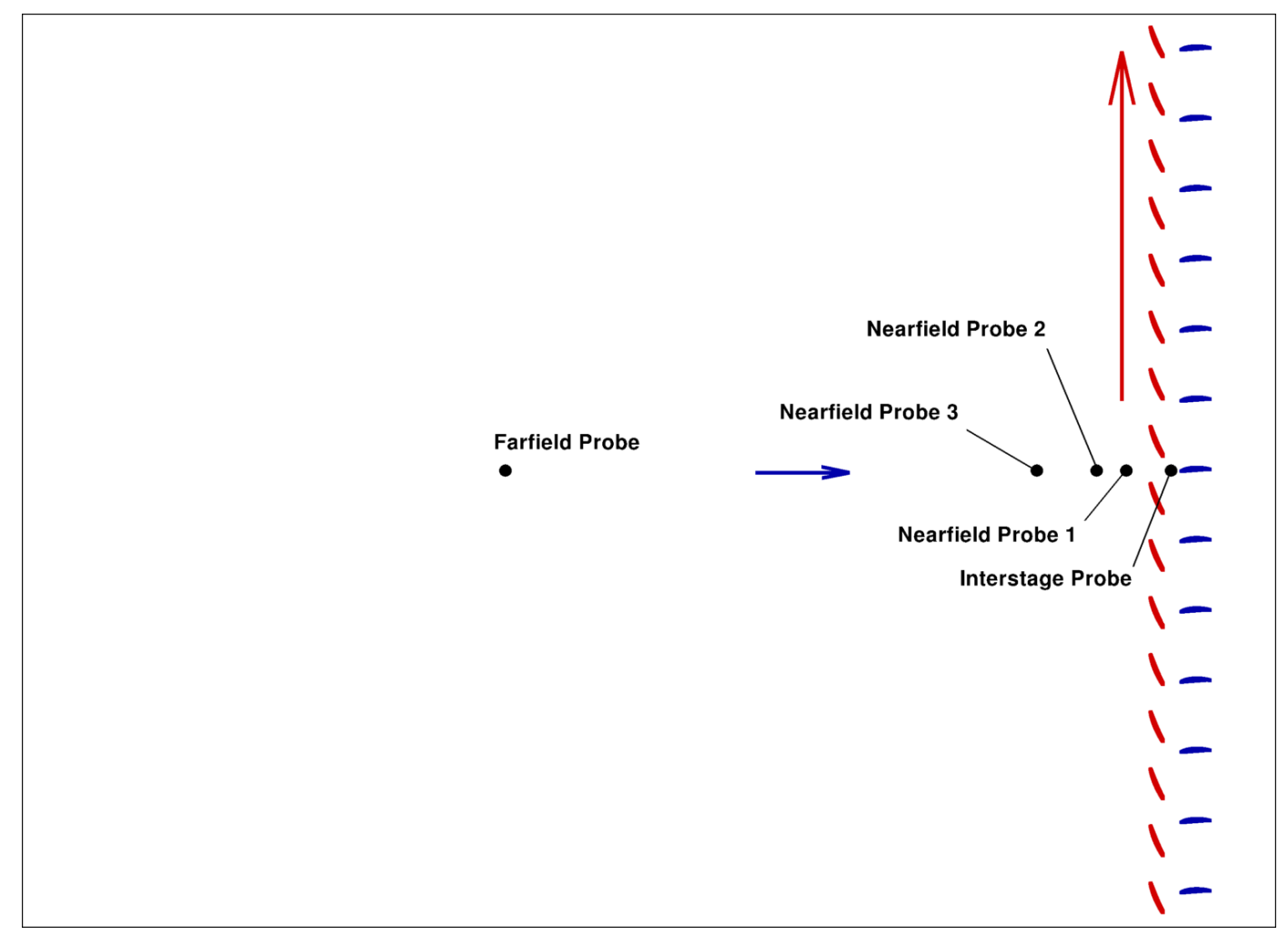

Figure 5.14: Computational domain with a regular Cartesian mesh.

The computational domain was increased after the subsonic case, now with 21 characteristic lengths in the horizontal direction in the regular region of the computational mesh and with the same 16 characteristic lengths in the vertical direction. The same mesh resolution is used here, with 300 control volumes through each characteristic length, resulting in 30,240,000 control volumes in the regular cartesian region and 31,780,400 total of control 
volumes including the stretch region for numerical damping of the reflecting acoustic waves. Figure 5.14 shows the pressure probes positioning through the computational domain. The computational domain with stretched control volumes is exactly the same as the subsonic cases.

\subsubsection{Transonic Rotor Only}

As seen in the subsonic case, the rotor alone is truly a case to validate the analytical theory regarding propagating modes. The case shown in figure 5.15 shows no acoustic modal structure being propagated at all.According to equation 2.130, the critical eigenvalue for each harmonic is $\sigma_{m 1, c}=15.2217 h$ and the Tyler-Sofrin [69] modes generated by the blades are $m=16 h$. When compared to the critical eigenvalue all the blade only modes are cut-off, showing exact phenomenon agreement between the analytical and numerical results.

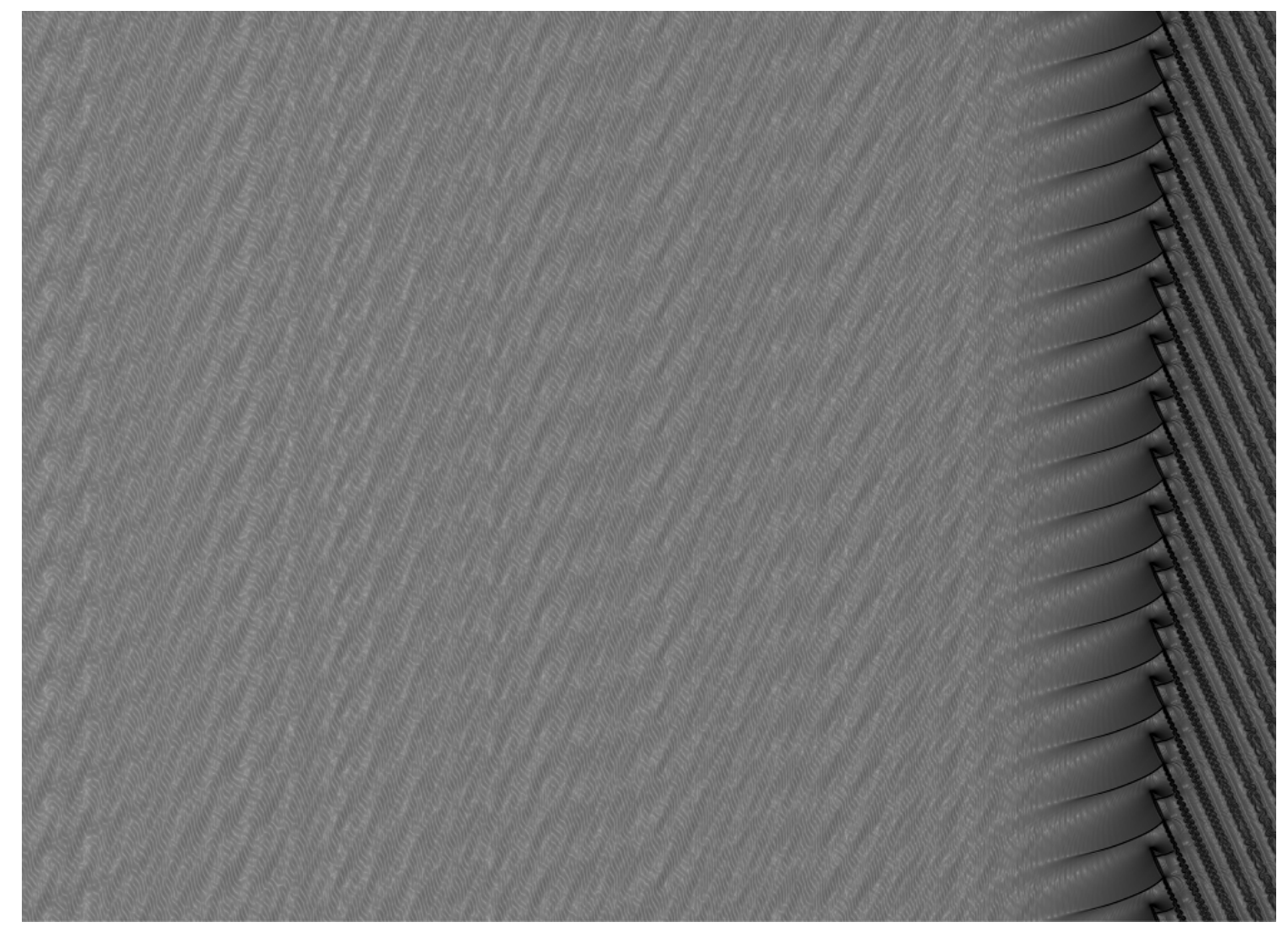

Figure 5.15: Visualization of the instantaneous aeroacoustic field generated by the transonic rotor cascade using the variable $\beta_{T}$.

The near field shows the shock waves at the pressure and suction sides of the rotor where 
it interacts with the wake produced by the neighbor preceding blade. Rotor self noise is produced on the trailing edge of each blade where it is propagated to the farfield.

According to the Tyler-Sofrin rule, the only acoustic modes produced by this case are the rotor only ones $m=16 h$ and the harmonics Figure 5.16 shows the nearfield pressure fluctuations due to the rotor blades passage and the frequency shift of the moving probe at each harmonic shows that there are only the rotor blade generated modes. The blade only generated modes are made mostly of the shock wave pressure jump discontinuity, as seen by the high values of RMS of pressure fluctuation in figure 5.17.

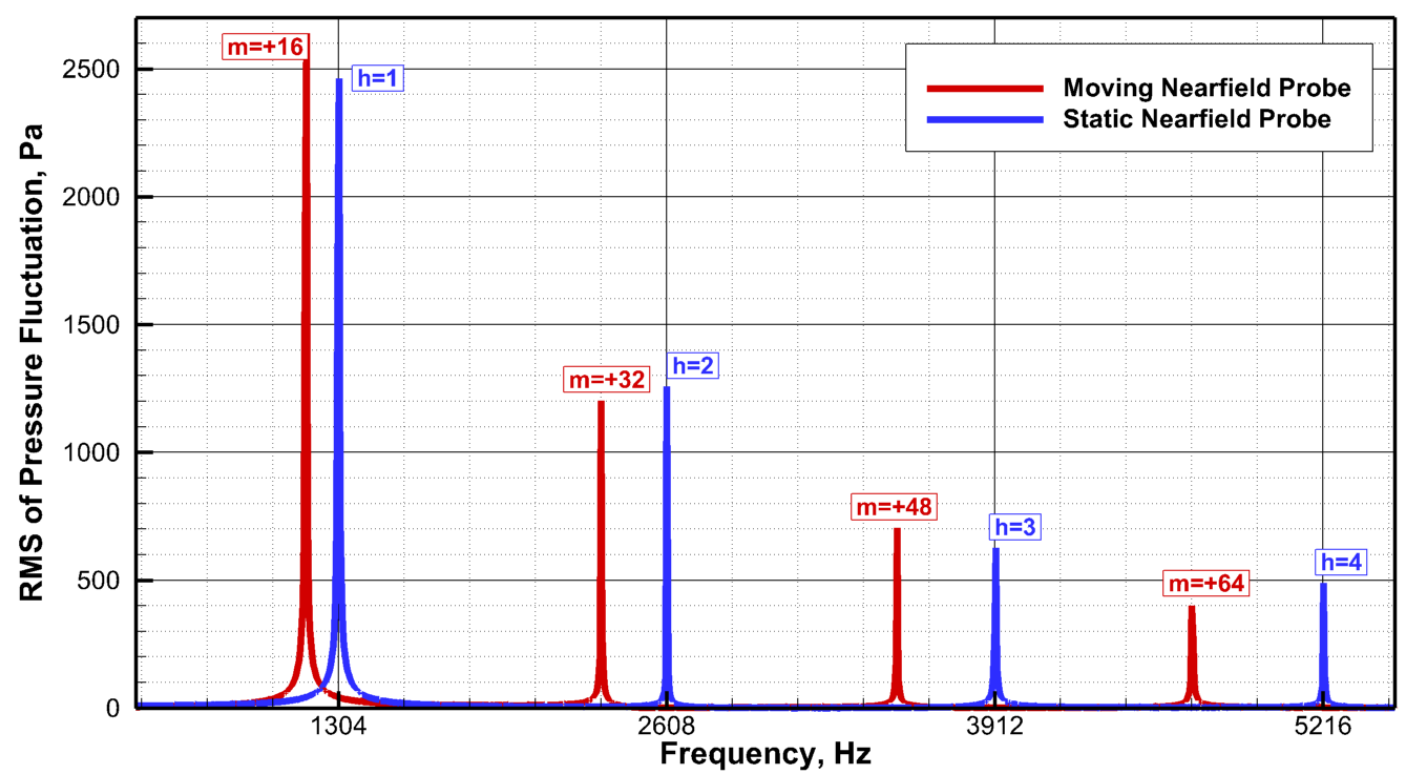

Figure 5.16: Spectra of the RMS value of the pressure fluctuations for the static nearfield probe 1 (blue) and the moving nearfield probe 1 (red) showing the decomposition of the nonlinear modes around each harmonic.

As seen in chapter 2, rotor only generated modes may propagate only if their phase speed, i.e. the rotor vertical speed, is approximately above the speed of sound. Figure 5.17 shows that the even though the RMS of pressure fluctuation amplitude is well above the usual acoustic ones due to the shock wave, there is a strong decay of the RMS of pressure fluctuations, implying that the generated modes are cut-off. 


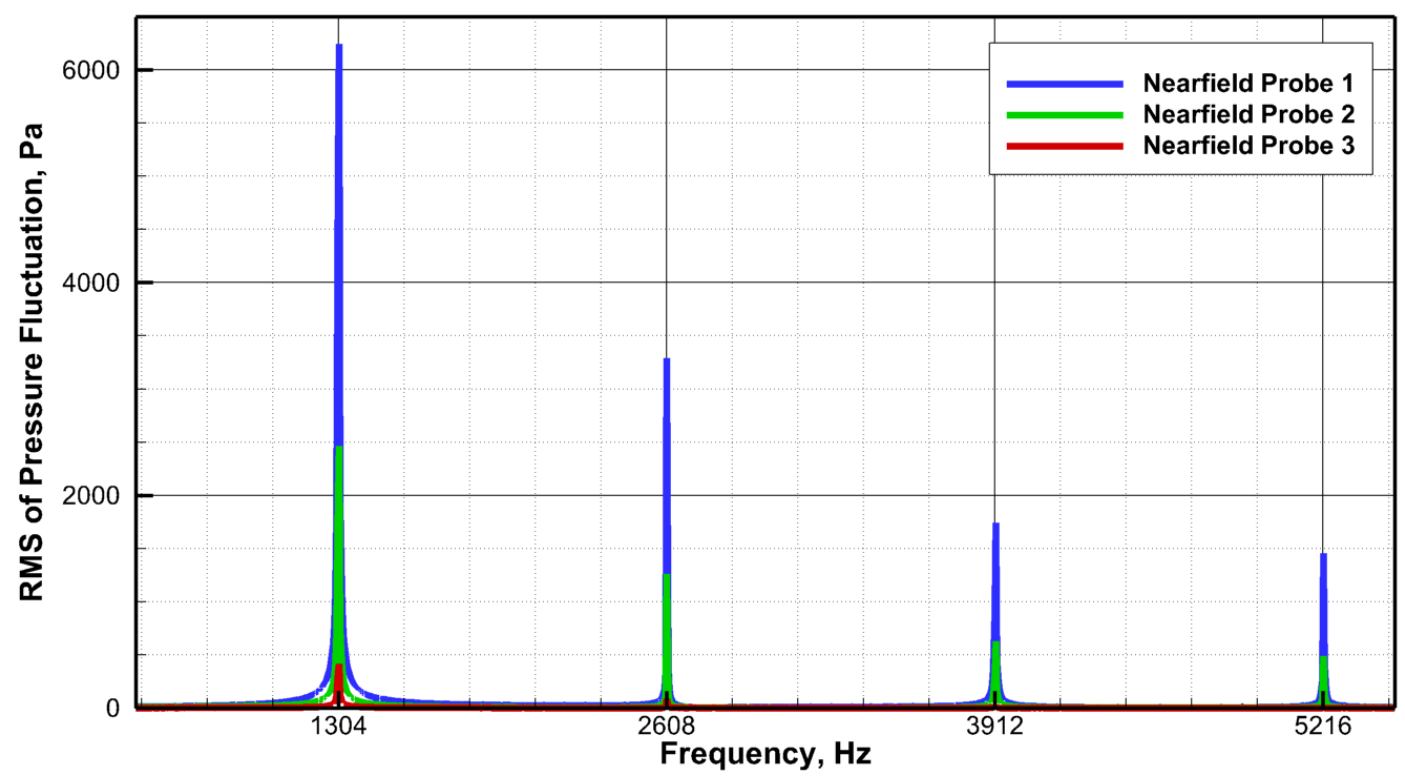

Figure 5.17: Nearfield probe pressure RMS signal decay due to the shock-waves evanescence.

\subsubsection{Transonic Rotor and Stator Interaction}

In order to correctly evaluate all the phenomena found on the transonic rotor-stator interaction case, we must first validate the case aeroacoustically. The mode generation and propagation theory will be used to assess the validity of the numerical results for the farfield, and when validated, the interstage will be analyzed from the viewpoint as a source of sound.

The rotor stator interaction case presents cut-on modes, unlike the rotor only cases, where though generated, the modes do not propagate. By combining the mode selection rule, the critical eigenvalue equation and the thin annular duct approximation, where $\sigma_{m 1}=$ $m$, one is able to know a priori all the modes propagated to the farfield. For the transonic blade with stator interaction case, the cut-on modes are described in table 5.3.

By using the Doppler effect to decompose the modes at each harmonic and comparing with table 5.3, a form of validation can be obtained for the farfield analysis. Figure 5.18 shows the mode decomposition, where the moving probe may decompose the modes that make a given harmonic. The probe velocity is a tenth of the rotor vertical velocity such that it is not fast enough for a mode of a harmonic will not overlap a mode from a neighboring 


\begin{tabular}{c|cccc}
\hline \hline $\mathrm{h}$ & 1 & 2 & 3 & 4 \\
\hline$f_{b p, h}(\mathrm{~Hz})$ & 1304 & 2068 & 3912 & 5216 \\
\hline$\sigma_{m 1, c}$ & 15,2217 & 30,4434 & 45,6651 & 60,8868 \\
\hline$m+$ & +3 & $+6,+19$ & $+9,+22,+35$ & $+12,+25,+38,+51$ \\
\hline$m-$ & -10 & $-20,-7$ & $-43,-30,-17,-4$ & $-53,-40,-27,-14,-1$ \\
\hline \hline
\end{tabular}

Table 5.3: Theoretical cut-on modes from the first to the fourth harmonic for the transonic rotor and stator cascade interaction case.

one.

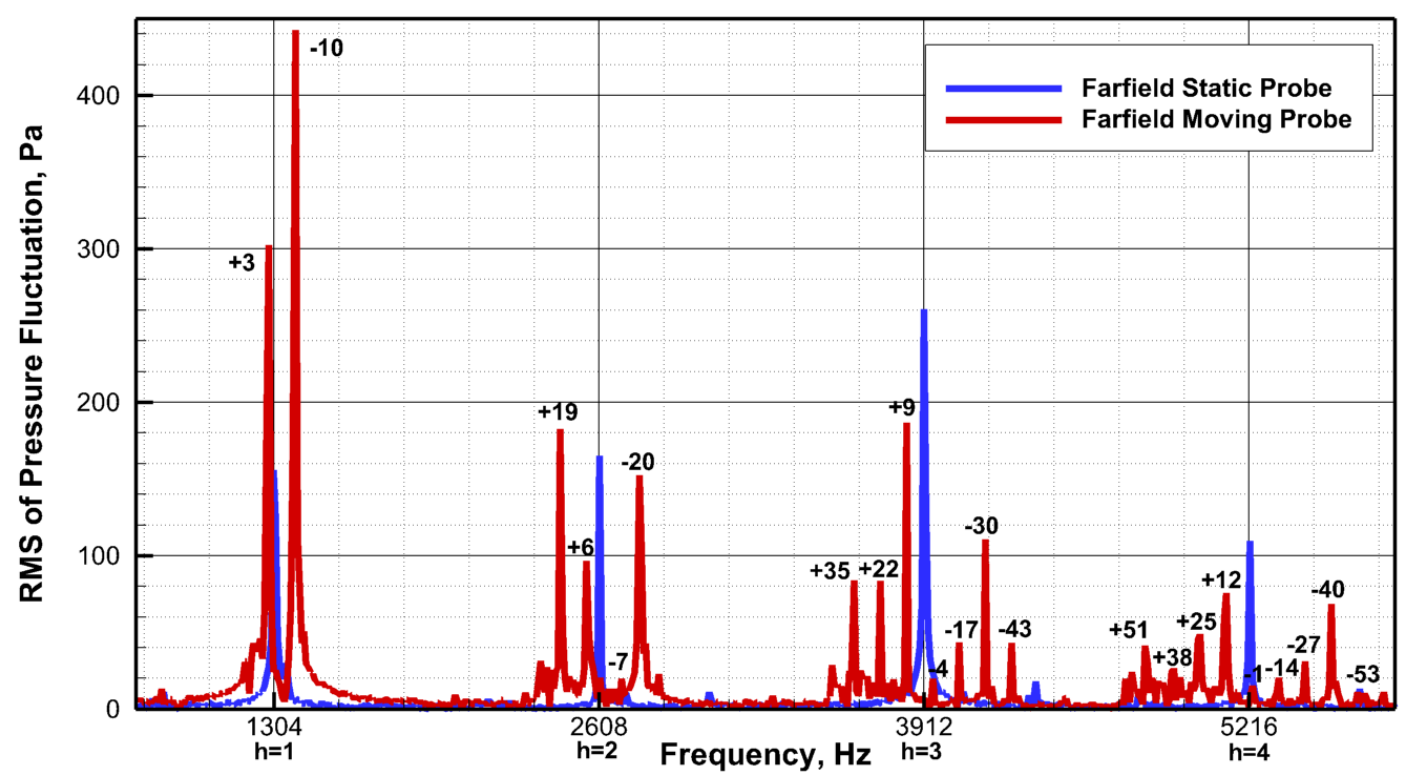

Figure 5.18: Pressure spectra of the farfield moving probe and the static probe, showing the modal decompostions up to the fourth harmonic.

Correct mode generation and propagation can be observed up to the fourth harmonic as shown in figure 5.18. Excellent results appear in the first and third harmonics with all predicted modes amplitudes well above the broadband content. In the second and fourth, some modes are on the level of the broadband with a slight prominence of their tone. It is worth noting that in the first and second harmonics, the modes amplitudes are higher than their respective harmonic, it happens because from the definition of the RMS pressure fluctuation, the modulus of the signal is used, thus not conserving the amplitude composing 
a given harmonic into its modes.

The second methodology used to validate the acoustic modal behavior is the angle measurement of the modes wavefront. The angles of the modes can be calculated theoretically and compared with the measured ones that appears in the $\beta_{T}$ visualization from figure 5.19. Unfortunately only the modes of the modes families $m=+3 h$ and $m=-10 h$ appeared in figure 5.19 due to their high amplitude when compared to the other modes, nonetheless the validation can be carried out. The measured modes angles show outstanding agreement with the theoretical ones, the error is 0.07 degrees for the $m=-10 h$ modes family and 0.02 degrees for the $m=+3 h$ modes family.

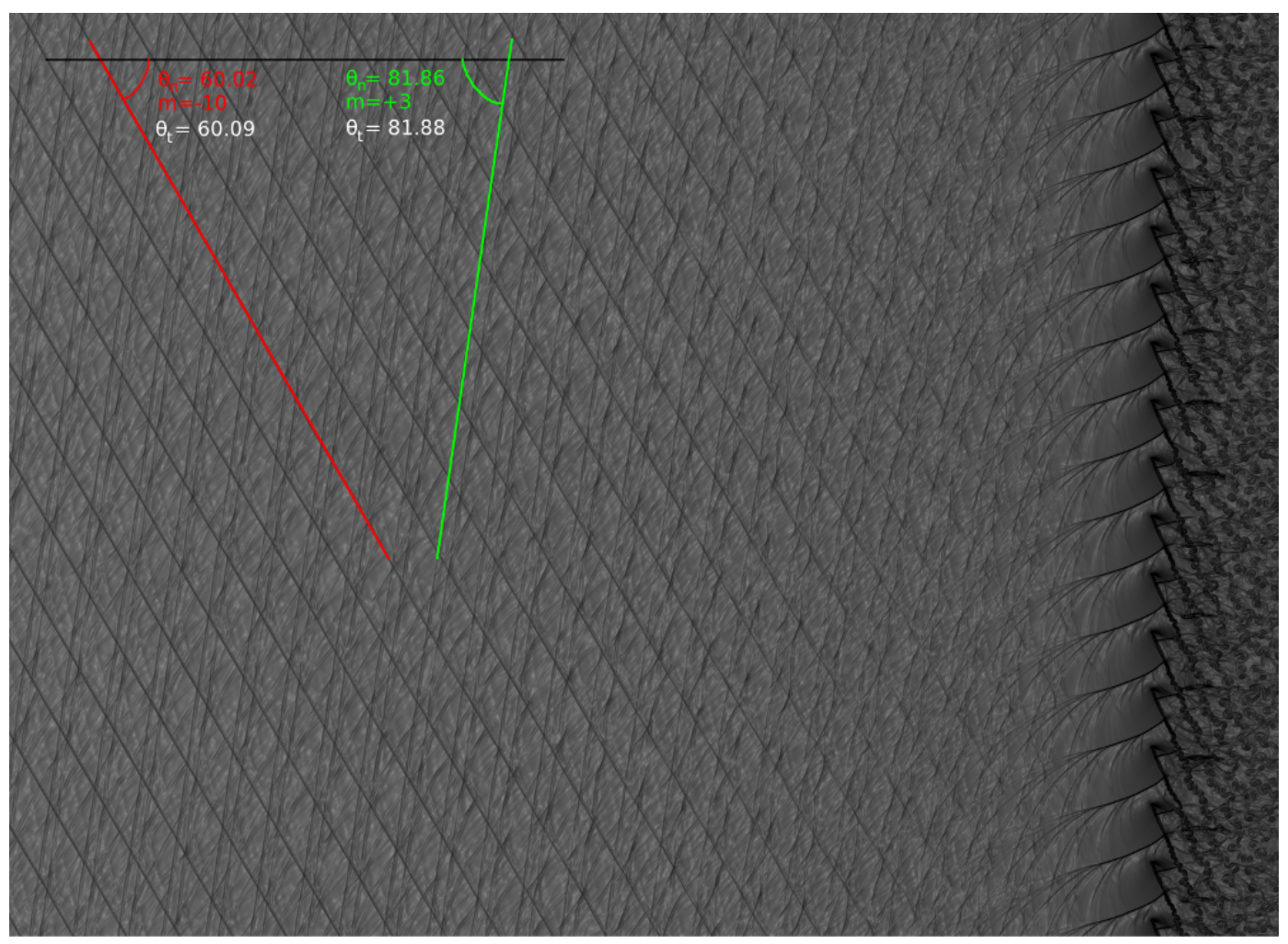

Figure 5.19: $\beta_{T}$ flowfield variable visualization with the modal angle measurement of the propagating wave fronts.

For the farfield mode propagation analysis the modes that were propagated are the correct ones according to the theory, making the sound source one of the correct solutions to tonal sound source of transonic rotor-stator interaction. Now the interstage region can be correctly assessed since it was validated as a correct sound source.

The $\beta_{T}$ field of the numerical solution for the rotor-stator interaction case shown in 
figure 5.19 depicts a very nonlinear and chaotic flow on the nearfield region; shock waves on both suction and pressure sides of the rotor interacting with the wake of the preceding rotor blade and self-noise being produced likewise in the rotor only case. The wakes produced by the rotor cascade and the moving shock waves interacts with the stator vanes cascade that keeps reflecting on the neighbor vanes. Due to the supersonic flow velocity before a shock wave, there is a sound barrier at the opposite flow direction, as can be seen in the rotor trailing edge region, creating a sound propagating channel that resembles a bat wing. Strong vorticity structures are convected with the flow at the downstream region.

Figure 5.20 shows the moving probes located at the rotor-stator interstage and at the nearfield upstream rotor region. Mode decomposition for the interstage probe shows that all the theoretical probes plus the rotor only shock wave modes that decay when propagated as shown in figure 5.18 and some cut-off modes that are generated as well according to the Tyler-Sofrin rule, are mainly negative valued (interaction) modes. The upstream nearfield probe shows that there is a silence region for most of the negative valued modes on the upstream nearfield region. After this silence region all the predicted cut-on modes appear and propagate through the farfield.

An important fact here is that even with a highly nonlinear and chaotic flow region at the interstage, the modes are generated correctly in a nonlinear regime, where the RMS amplitudes are at least an order of magnitude higher than of the modes propagated in farfield region. For a correct rotor-stator interaction tonal noise, it is not necessary that the terms regarding the flow in the interaction region must be in the linear range. Tonal modal response may exist in a source where shock-waves and high amplitude pressure fluctuations are part of the solution, as can be seen in figure 5.21. We therefore may classify the source region as nonlinear and the propagation region after this silence region as linear, considering mode amplitudes. The modes generated by the transonic rotor-stator interaction are even better defined than the ones found in the subsonic rotor-stator interaction. 


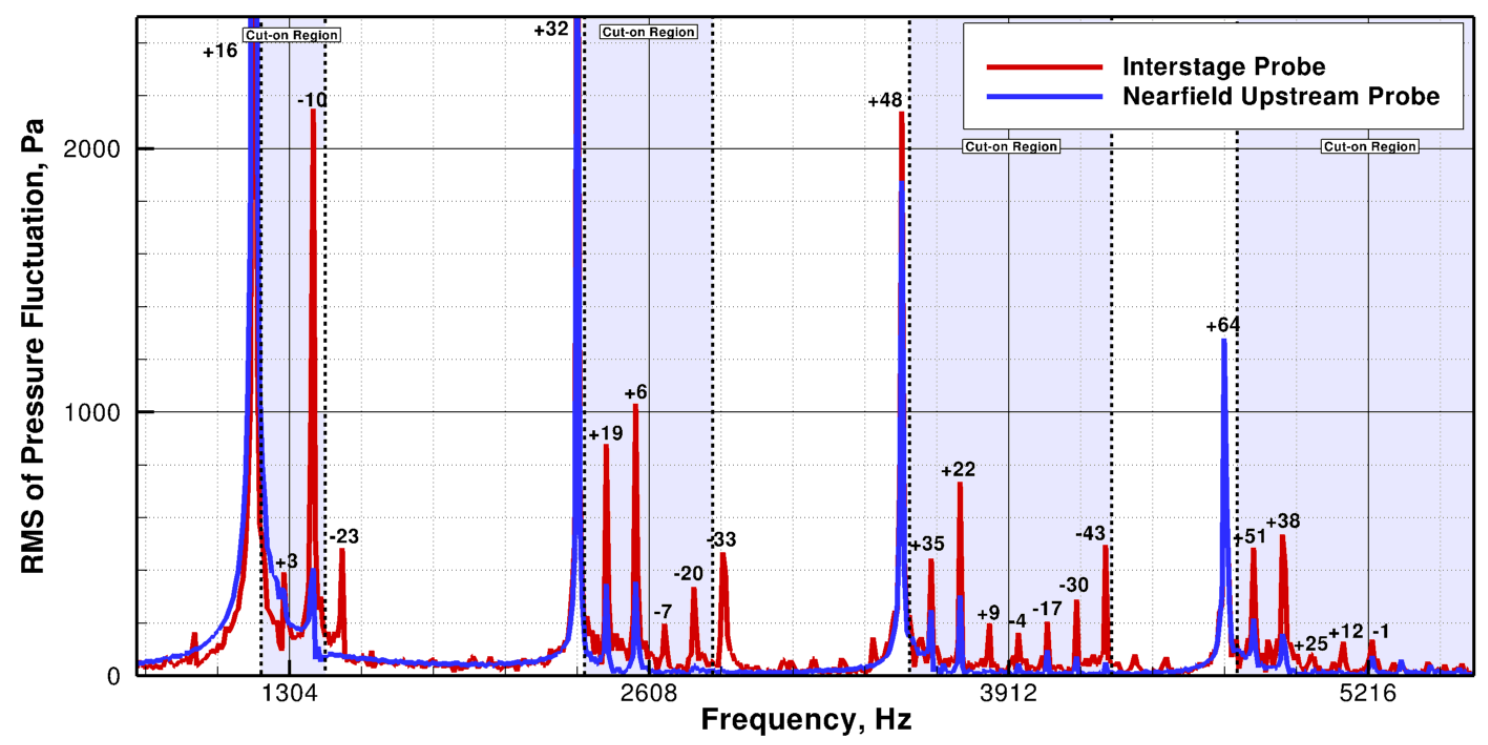

Figure 5.20: Spectra of the RMS value of the pressure fluctuations for the moving interstage probe (red) and the moving nearfield probe 1 (blue) showing the mode decomposition around each harmonic, being all the identifiable modes given by the Tyler-Sofrin rule. The light-blue regions delimited by dashed lines are the cut-on regions around each harmonic. 


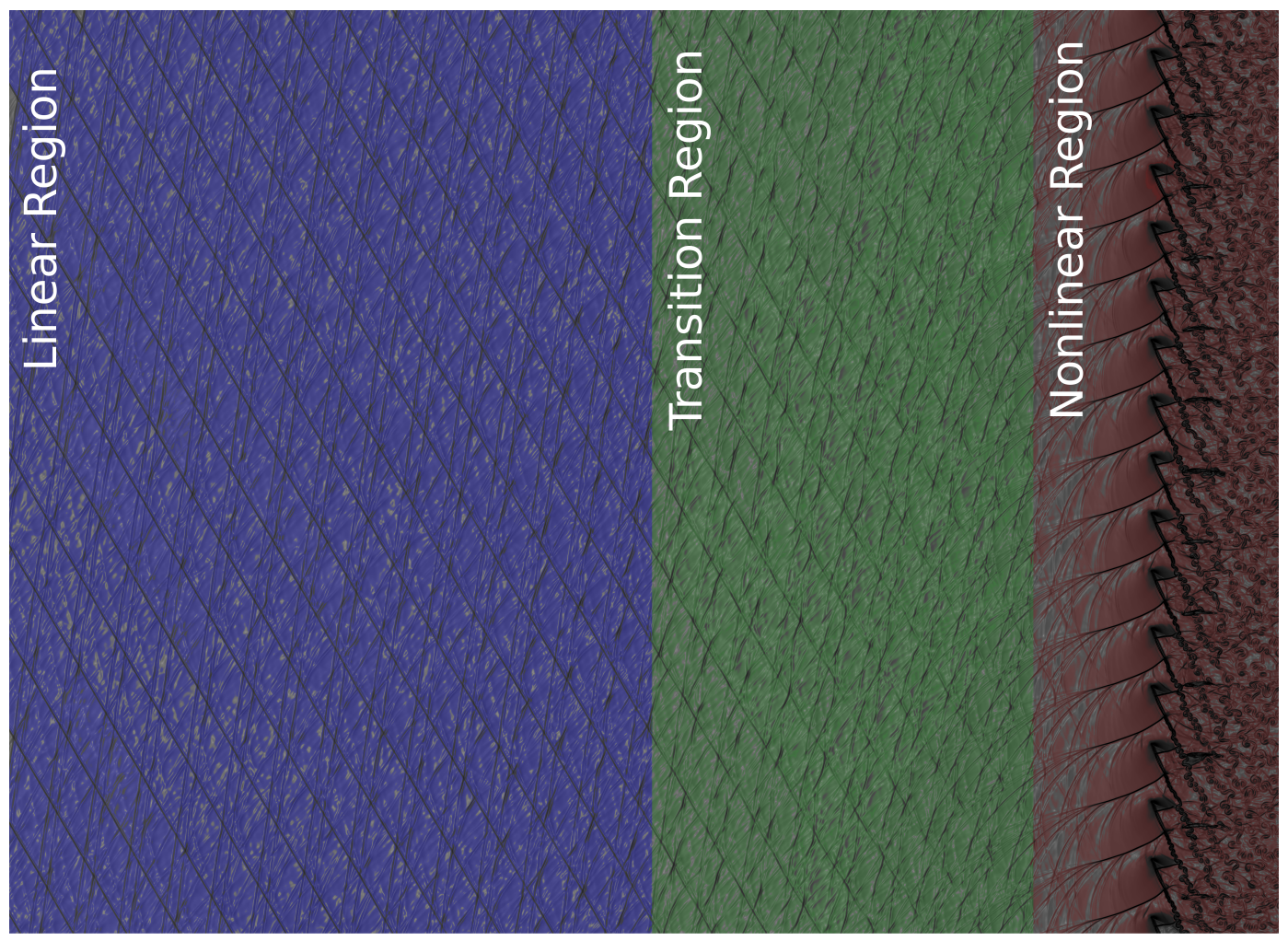

Figure 5.21: Linear, transition and nonlinear regions of the computational domain. The dominant linear modes $\mathrm{m}=-10$ and $\mathrm{m}=+3$, associated to the first harmonic, are easily identified in the linear region. In the transition region it is possible to observe the superposition phenomena of nonlinear waves that will result in the linear modes. Transonic shocks and shock-wake interactions are observed in the nonlinear region. 


\subsubsection{Rotor and Stator Cascade Simulations in Supersonic Regime}

Since the buzz-saw noise can be regarded as a two dimensional phenomenon through the duct, i.e. there are no effects in the radial direction, its flow can be analyzed in the region near the duct wall [79]. The approach used in this work is the two-dimensional rotor blades and stator vanes cascades flow simulation then remains valid.

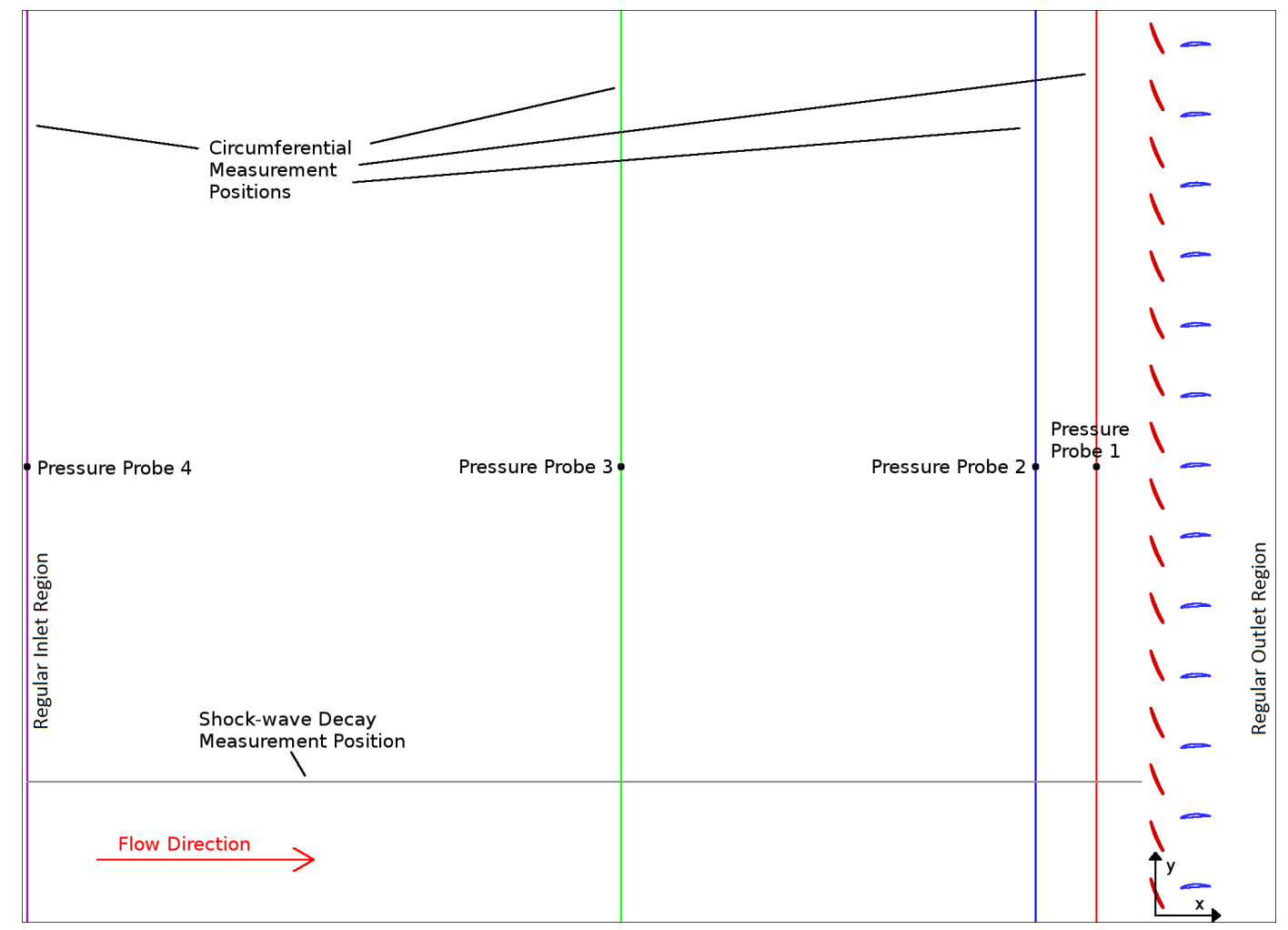

Figure 5.22: Computational domain of regular control volumes showing the rotor blades and stator vanes cascades positioning, the pressure probes and circumferential measuring locations.

The computational domain for the supersonic cases is exactly the same used for the transonic cases. The flow conditions used in all cases are the following: the horizontal flow velocity is $U_{\infty}=137.97 \mathrm{~m} / \mathrm{s}$, the rotor blades upward velocity is $U_{B}=381.7 \mathrm{~m} / \mathrm{s}$, which gives a blade passing frequency of $1594 \mathrm{~Hz}$. The flow inlet pressure is of $p_{\infty}=101,300 \mathrm{~Pa}$ and the flow inlet temperature is $T_{\infty}=300 \mathrm{~K}$. The resulting Mach numbers are $M_{\infty}=$ 0.3972 and $M_{B}=1.1$. All these flow conditions are from an inertial reference frame fixed 
at the stator vanes cascade.

In the vertical (circumferential) direction there are 16 units and 21 on the horizontal (axial) direction. The probes (black dots) and circumferential measurements (colored lines) are shown in figure 5.22, they are at 1, 2, 9 and 19 units of distance in the horizontal direction from the rotor blades cascade. The cascades configuration used in the numerical simulations are of 16 rotor blades and 13 stator vanes and the origin of the coordinate system is at mid chord of the rotor blades cascade.

\subsubsection{Supersonic Rotor Alone in Nominal Conditions}

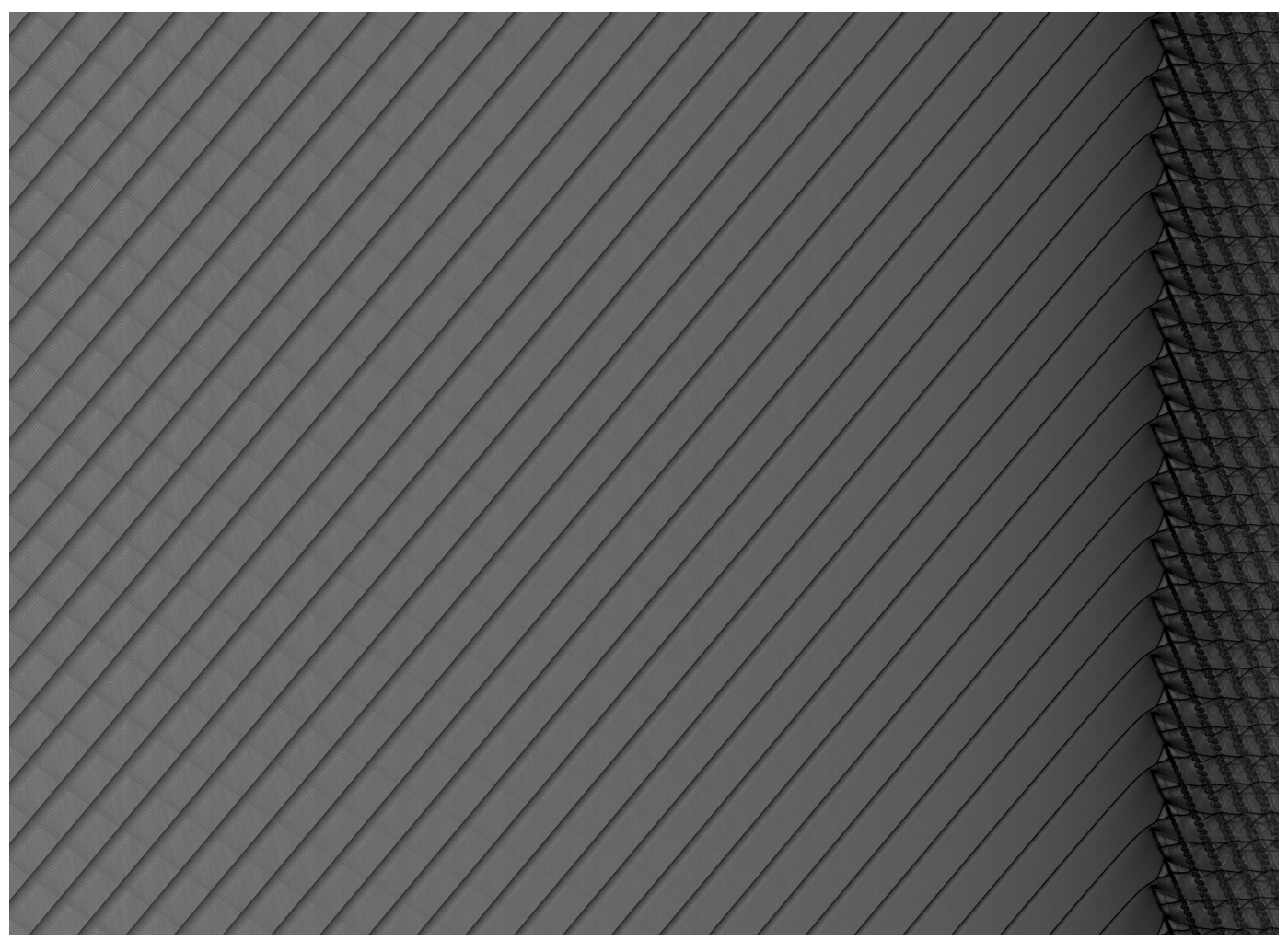

Figure 5.23: The $\beta_{T}$ flow visualization of the supersonic nominal rotor blade cascade alone configuration.

The flow visualization in a developed flow state is shown in figure 5.23. At the flow outlet there is a region of interaction between the rotor blades vorticity, generated by compressibility effects, explained in appendix IV, and the system of rotor locked shockwaves. At the inlet direction there is a system of shock-waves that moves along with the 
rotor blades cascade and that decays rapidly at the horizontal direction.

The first validation of the rotor blades cascade at supersonic condition is the pressure probes analysis. No signs of blade passing frequency decomposition into rotor shaft turning frequency may appear in the pressure probes. Indeed it does not happen as shown in the spectra of the four pressure probes in figure 5.24. The tonal noise is distributed solely on the blade passing frequencies, concluding that in a time sense the pressure distribution and shock-wave propagation at each rotor blade is perfectly even.
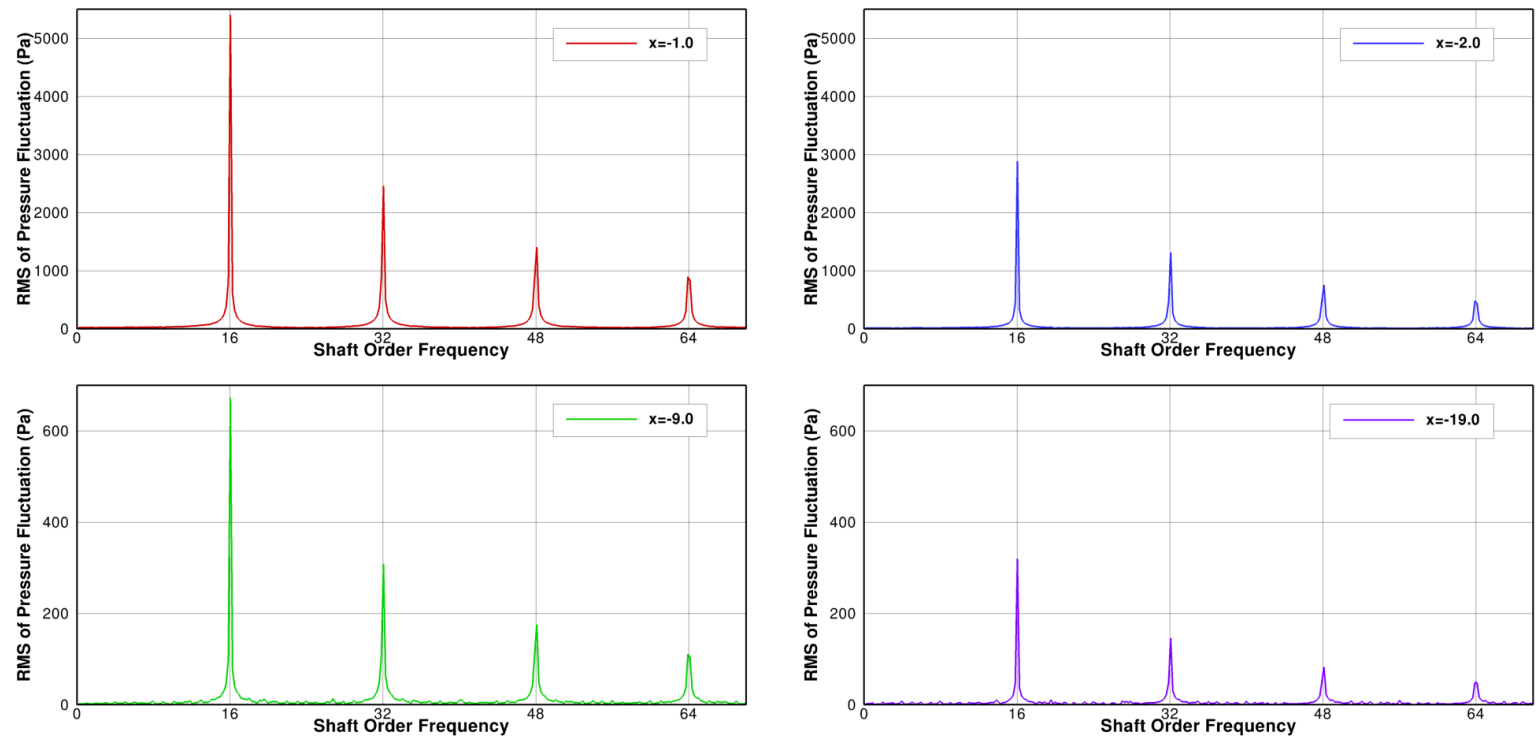

Figure 5.24: Spectra up to the fourth harmonic of pressure probes from the supersonic nominal rotor blades cascade only case.

The second validation for the moving immersed boundary methodology for supersonic cascades can be regarded as the spatial correctness of the flow. There can be absolutely no flow distortions, nor differences between the flow pattern at each rotor blade in the system of shock-waves if there are no rotor blade-to-blade geometrical differences. The pressure coefficients extracted from the circumferential stations 1 to 4 are shown in figure 5.25. The pressure coefficient distribution for the circumferential station 1 shows strong sawtooth pressure discontinuities due to the shock-waves passage, where each pressure profile is exactly the same of its neighbors. This trend remains the same for the whole inlet flow-field until the fourth circumferential station, where decay is present but the pressure profile remains the same for all the blade-to-blade shock-waves. 

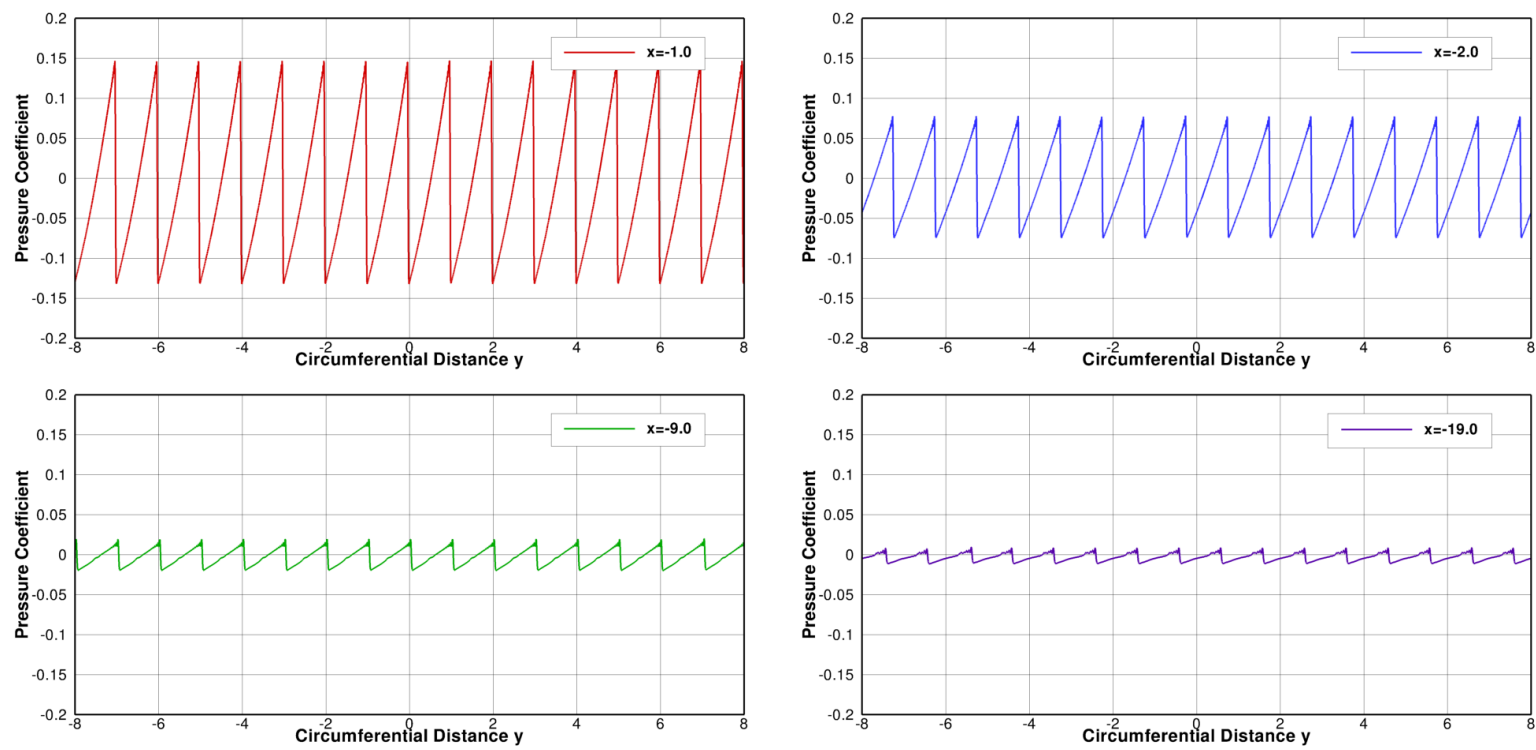

Figure 5.25: Pressure coefficient profiles of the circumferential stations for the supersonic nominal rotor blades cascade only configuration.

The last validation of this case is the sawtooth pattern decay along the axial direction. According to equation 2.147 there is a pressure decay of the sawtooth wave pattern along the duct axis proportional to $1 / x$ at large distances from the rotor. The pressure coefficient distribution along the horizontal direction from the rotor blades cascade towards the inlet is shown in figure 5.26 with the theoretical scaled decay. There is an excellent agreement of the sawtooth wave amplitude between the numerical result and the analytical theory, up to the end of the regular mesh region. The shape of the sawtooth wave also shows good agreement with the theoretical shape.

With a qualitative and quantitative validation of the immersed boundary methodology, concerning the behavior of the flow field with rotor blades at supersonic speeds, more realistic cases are verified and their more complex physics is assessed with the numerical simulations. The first one is the case where the rotor blades are still identical and the stator vanes cascade interacting with the rotor blades cascade is added. In the second case there are small geometrical differences between the rotor blades and finally the case where the rotor blades cascade with geometrical differences interacts with the stator vanes cascade. 


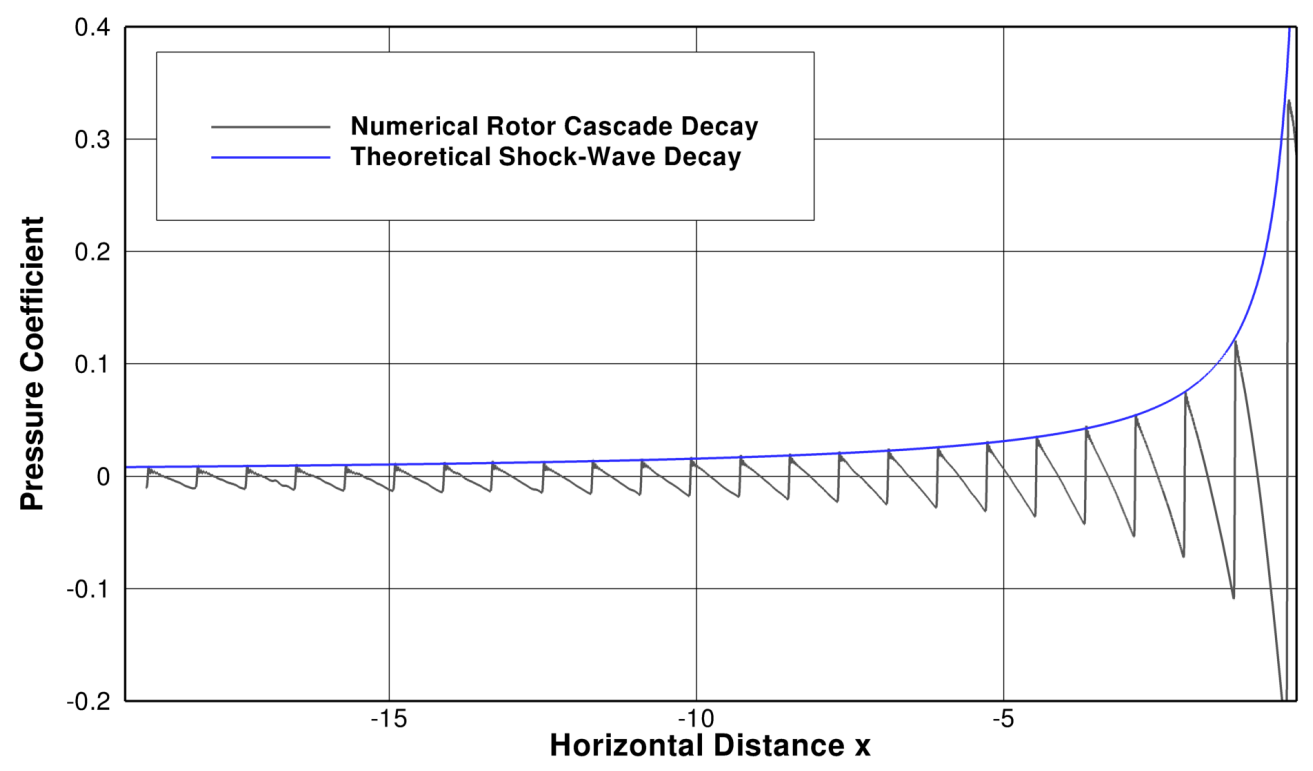

Figure 5.26: Pressure coefficient decay of the sawtooth pattern in the $\mathrm{x}$ direction for the supersonic nominal rotor blades only configuration.

\subsubsection{Supersonic Rotor and Stator Interaction in Nominal Conditions}

The following case presented here is the interaction between the rotor blades and stator vanes cascade. Comparing figures 5.23 and 5.27 the general characteristics of the flow remains almost the same but with slight differences. The system of shock-waves generated by the rotor blades supersonic tangential speed still has its perfectly ordered spacing and decay. The general shape of the shock-waves near the rotor blades also remains the same. Some differences now arise from the stator vanes and rotor blades interaction. The wake of the rotor blades interact with the stator vanes similar to the subsonic and transonic flow conditions. Strong shock-wave reflections between the stator vanes are also present in the flow outlet region. Between the propagating shock-waves at the inlet direction, there are signs of the effects of rotor blades and stator vanes interaction, where some modal response perpendicular to the shock-waves can be observed.

Considering the spectra of the time pressure probes from the first validation case with the rotor blades and stator vanes cascades case in figures 5.24 and 5.28 it can be seen that there is not any differences in the probes signal. The shock-waves intensity remains almost 
the same between both cases and the tonal blade passing frequency remains the only content on the signal.

By comparing the pressure coefficient extracted from the circumferential stations 1 to 4 of the nominal rotor blades cascade alone and the case with the stator vanes cascade in figure 5.29, some slight differences occur between cases. Although not seen in the spectra of the time pressure probes, there is some small amplitude variations between each sawtooth wave. On stations at $x=-9$ and $x=-19$ there is some degeneration of the sawtooth wave shape, caused by the rotor blades and stator vanes interaction. The influence of the stator vanes on the sawtooth waves does not changes its amplitude, nor the signal phase, but adds some wave-like content to the signal. While the stator vanes cascade presence causes some degeneration of the expansion waves between the shock-waves, its influence is not sufficient to produce disturbances on the shock-waves signature to cause multiple pure tone noise and its related phenomena such as shock coalescence.

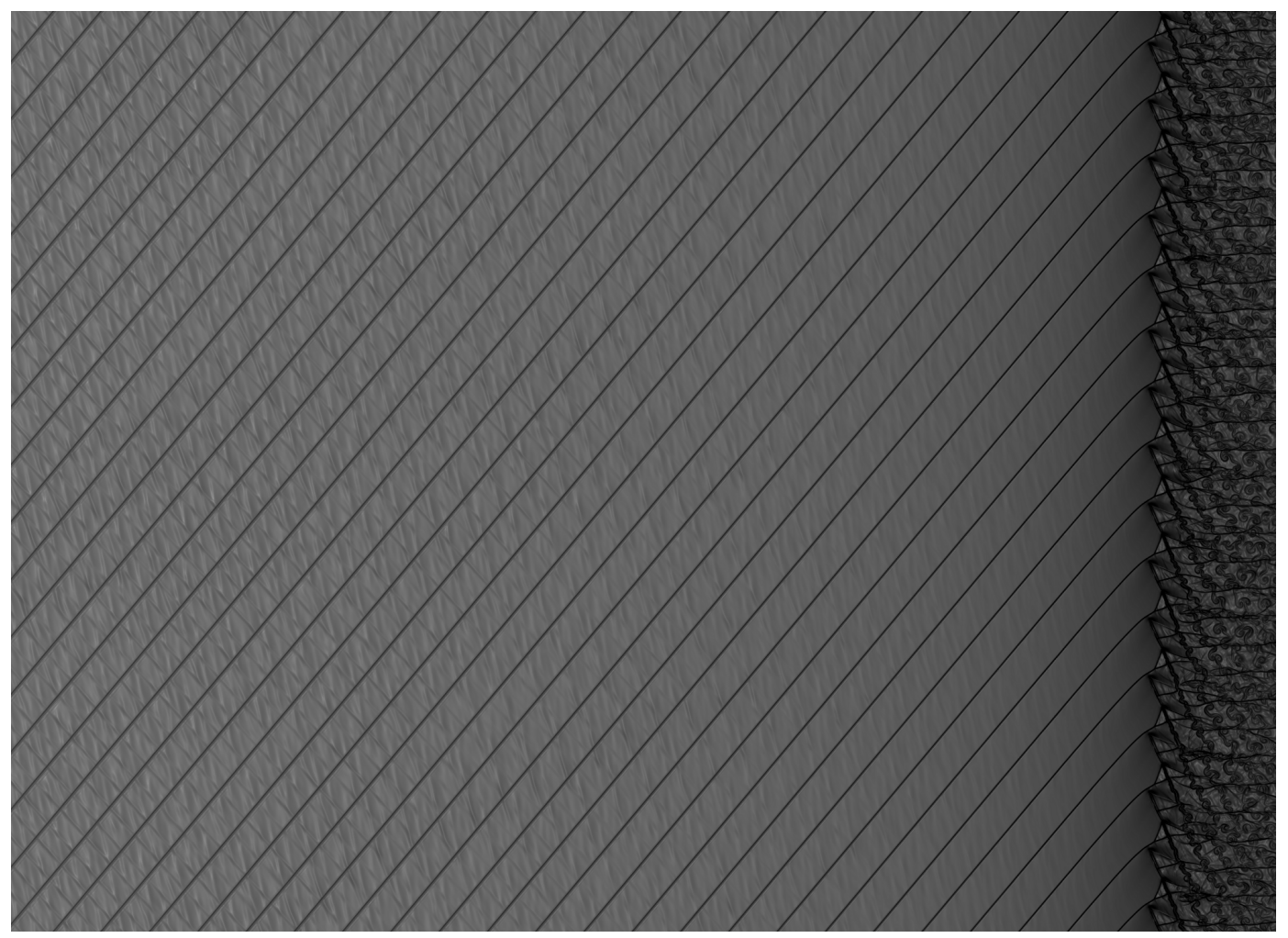

Figure 5.27: The $\beta_{T}$ visualization of the supersonic nominal rotor blades and stator vanes cascades configuration.

Figure 5.30 shows the comparison between the theoretical and numerical decay of the 

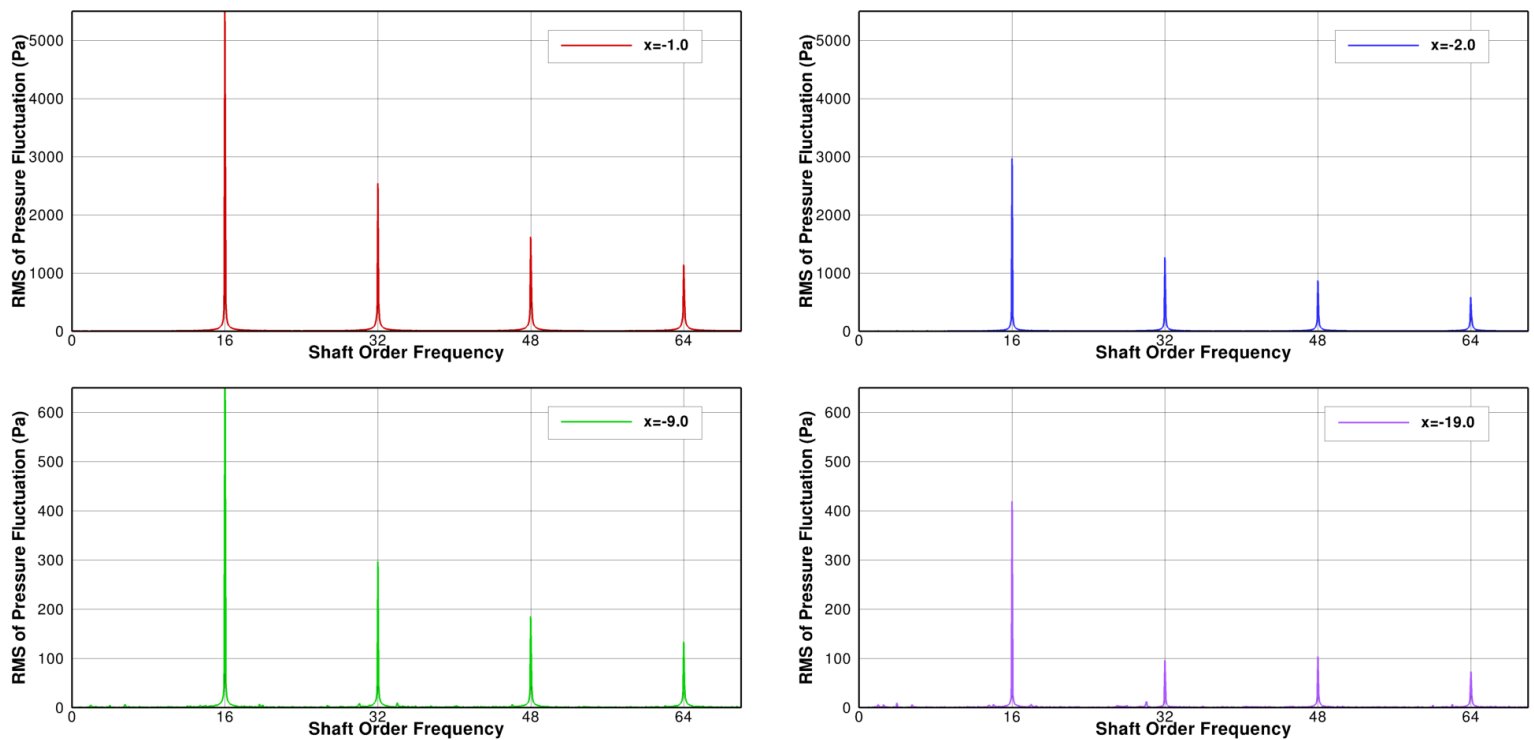

Figure 5.28: Spectra up to the fourth harmonic of pressure probes from the supersonic nominal rotor blades and stator vanes cascades case.
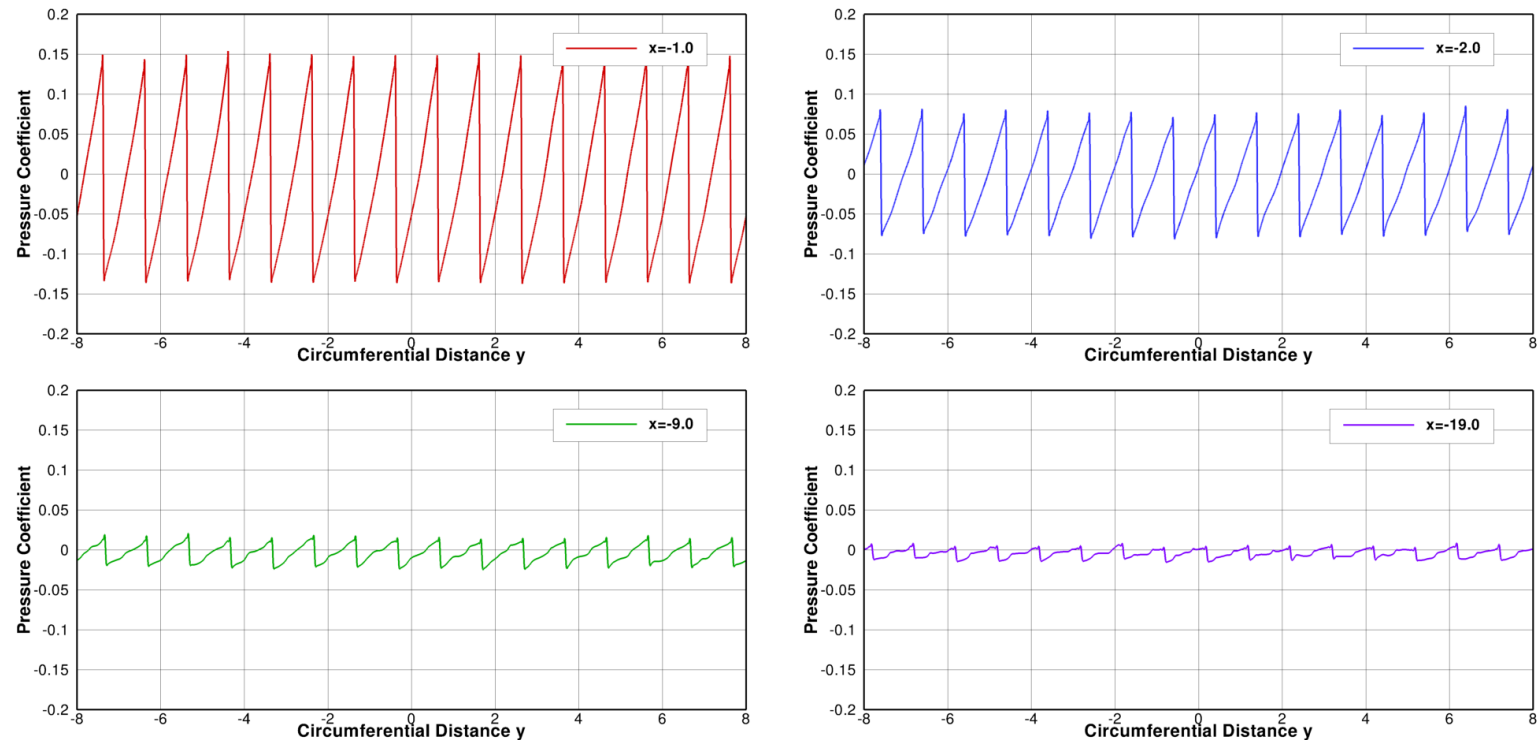

Figure 5.29: Pressure coefficient profiles of the circumferential stations for the supersonic nominal rotor blades and stator vanes cascades configuration.

sawtooth wave amplitude in the axial direction. It is seen that there is still an excellent agreement between the numerical and theoretical results. The influence of the stator vanes cascade may be noted on the slight degeneration of the sawtooth waves shape, specially when the sawtooth wave achieves a slow decrease on its amplitude around $x=-5.0$. 


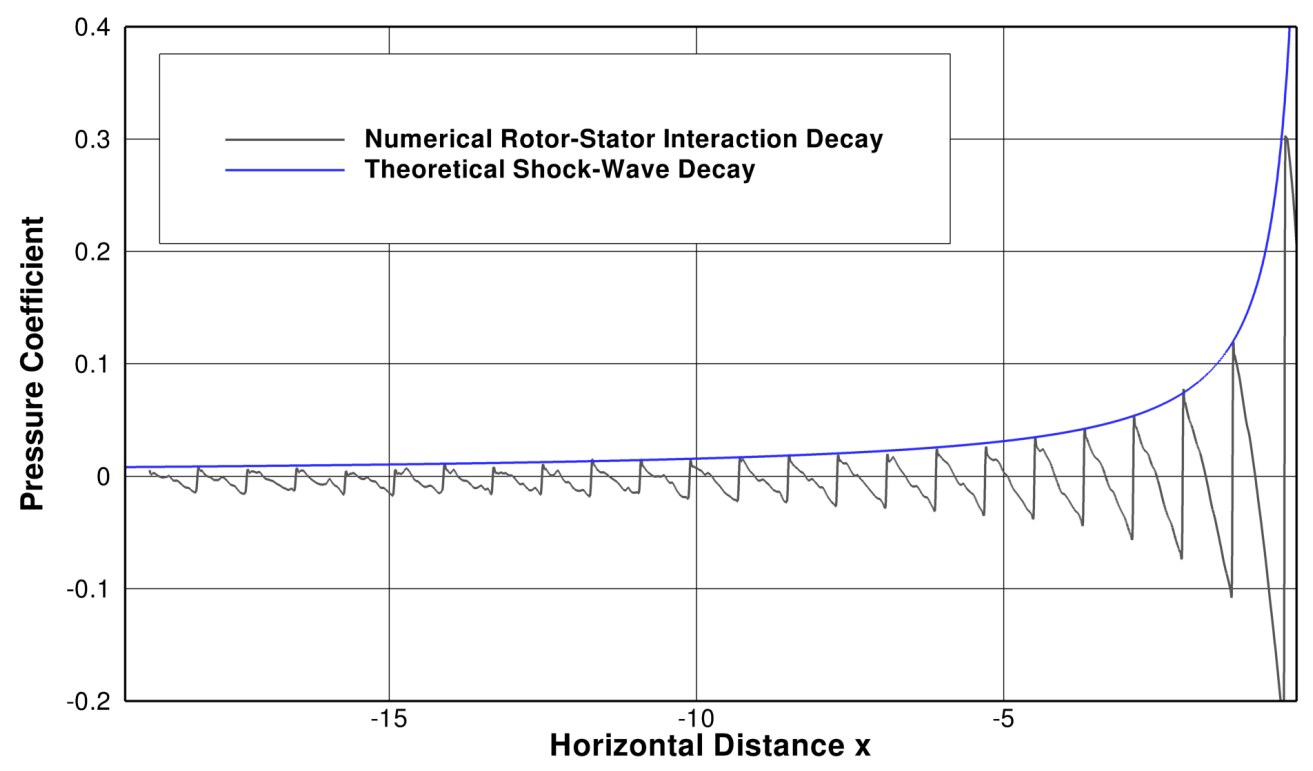

Figure 5.30: Pressure coefficient decay of the sawtooth pattern in the $\mathrm{x}$ direction for the supersonic nominal rotor blades and stator vanes cascades configuration.

\subsubsection{Supersonic Rotor Alone in Realistic Conditions}

In the rotor blades cascades with nominal conditions considered in previous sections, the generated and propagated sawtooth pressure field from the supersonic rotor blades cascade is identically repeated from blade to blade, with the consequence of having a fixed tonal frequency related to each passing of the rotor blades on the generated noise. We now consider the realistic case where due to extended use and manufacturing characteristics there are some geometrical non-uniformities at blade-to-blade on the rotor row. These differences may affect the stagger angle, camber, thickness and blade spacing. Theoretical parametric studies [79] were carried out, where the effect of blade-to-blade changes in stagger angle and pitch distance within manufacturing tolerances was assessed. He found out that the variation of stagger angle was the most significant geometric non-uniformity to induce the MPT noise. A variation of \pm 0.75 degrees more or less for the stagger angle error was sufficient to produce well noted MPT noise in the pressure spectrum and changes in spatial pressure profiles as well.

The modification on the stagger angles for each rotor blade is shown in table 5.4. The 


\begin{tabular}{c|ccccccccccccccccc}
\hline \hline Blade Number & 1 & 2 & 3 & 4 & 5 & 6 & 7 & 8 & 9 & 10 & 11 & 12 & 13 & 14 & 15 & 16 \\
\hline Stagger Variation & - & + & + & - & + & - & - & - & + & + & + & - & + & - & + & - \\
\hline \hline
\end{tabular}

Table 5.4: Randomly generated stagger error distribution for the rotor blades cascade in realistic conditions.

plus sign means that a value of 0.75 degrees is added to the nominal stagger angle and the negative sign means that 0.75 degrees is subtracted from the nominal stagger angle. The distribution is randomly generated and evenly distributed between positive and negative values.

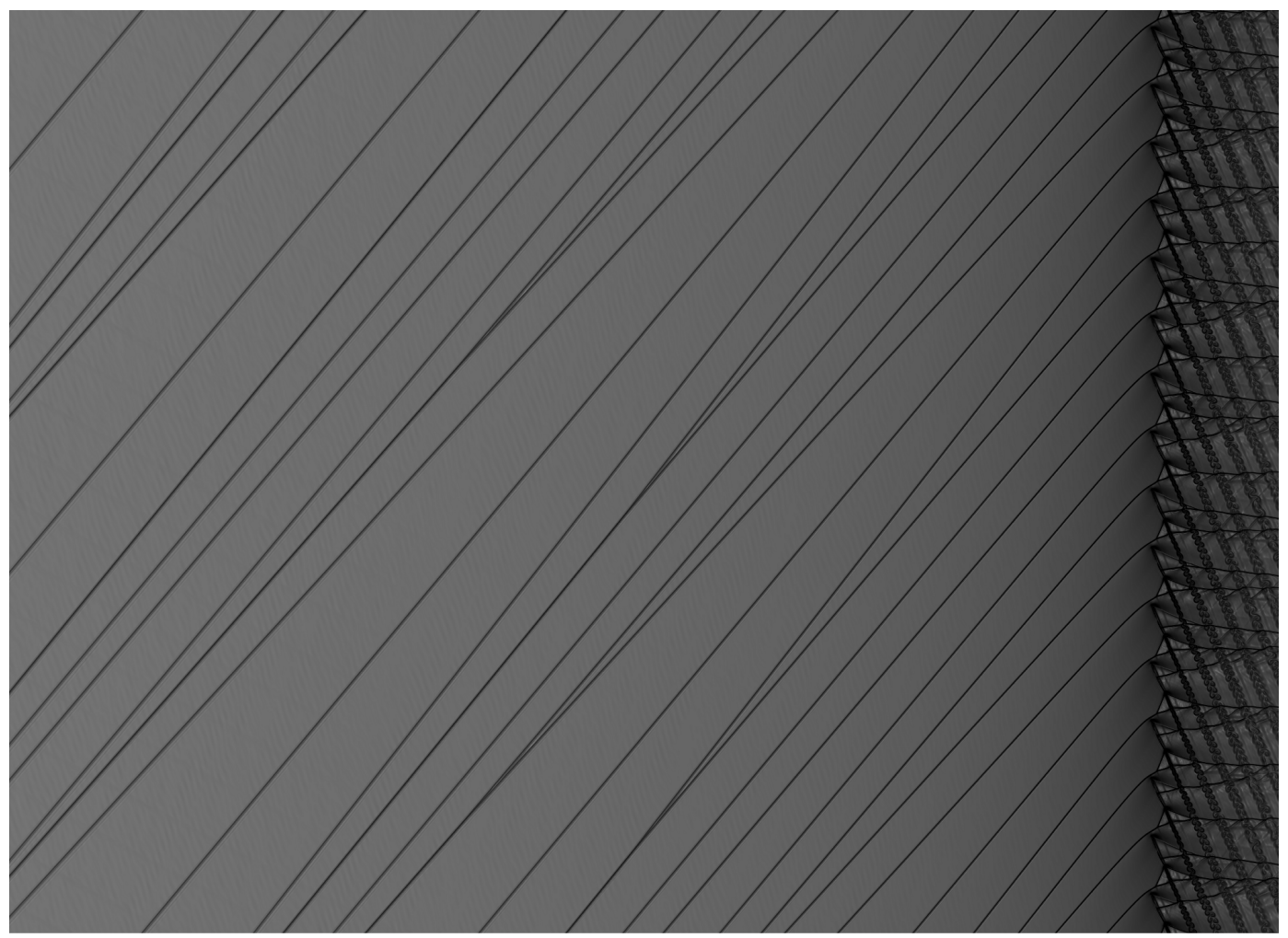

Figure 5.31: The $\beta_{T}$ visualization of the supersonic realistic rotor blades cascade configuration.

The first result concerning the effects of stagger error distribution between rotor blades is shown in figure 5.31. Visually, near the rotor blades in the upstream direction there is not much influence of the imposed stagger error. As the generated shock-waves propagate through the flow in the inlet direction, the difference of the shock-waves of each rotor blade grows until they encounter and merge with each other, resulting in a single shock-wave. 
This trend continues to occur until the end of the computational regular region, depicting the buzz-saw effect on the flow field.
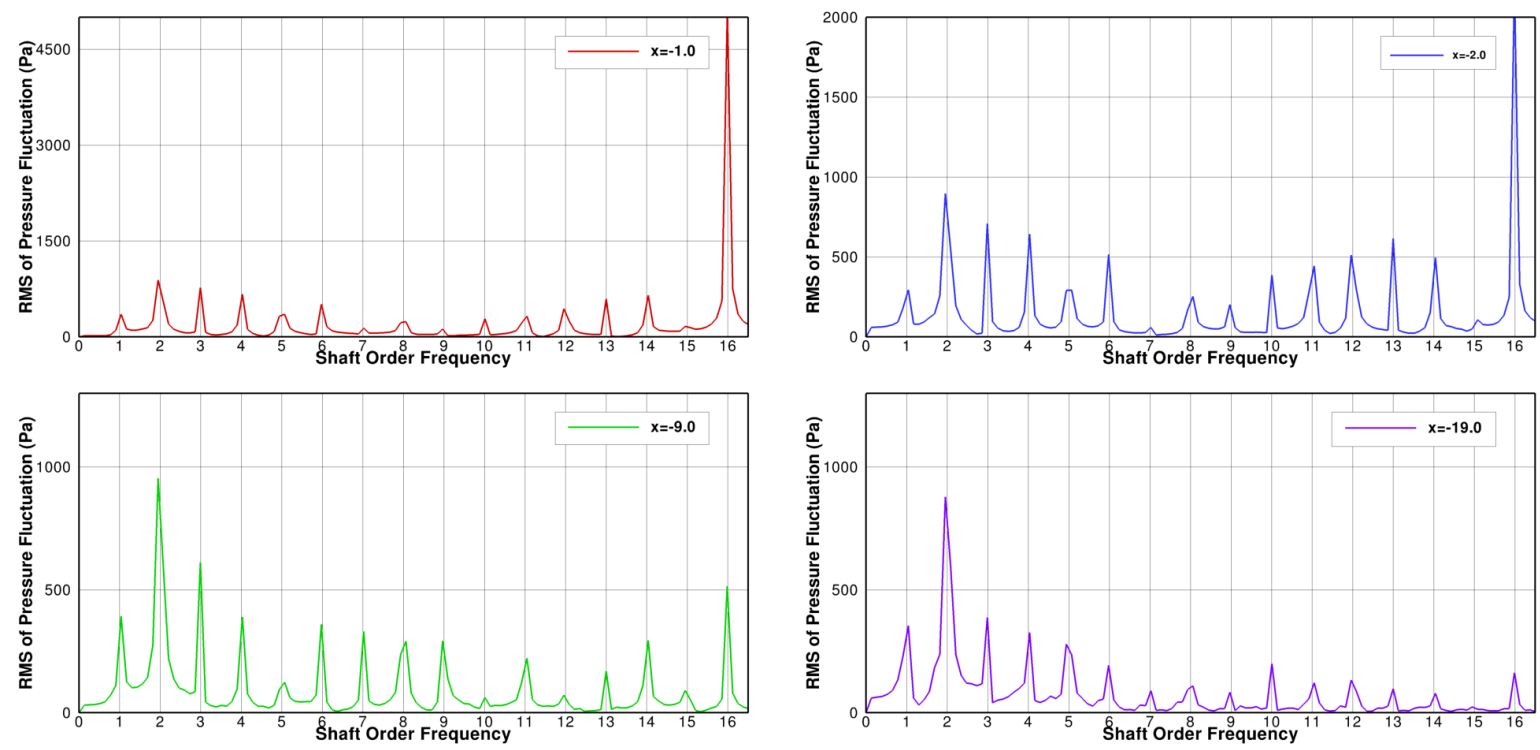

Figure 5.32: Spectra for the first harmonic of pressure probes from the rotor blades cascade case showing the multiple tone noise generation from shaft order 1 to 16 , being the later equal to the blade passing frequency.

Figure 5.32 shows the spectra of the pressure probes located at the circumferential stations. Multiple pure tone harmonics are shown for the first blade passing frequency harmonic. The correct number of tones is present in the probes of all axial stations, where it is given by the number of the rotor blades. For the pressure probes located at the first two circumferential stations $(x=-1.0$ and $x-2.0)$, the strongest tone is the one related to the blade passing frequency. In station at $x=-9.0$ the first two shaft order harmonics begin to prevail over the blade passing frequency and this trend is observed at station $x=-19.0$ as well. This is due to the uniformity of strength and spacing of the shockwaves close to the rotor, but as the axial distance grows, they become more irregular. This energy spectrum exchange is also observed in experimental observations $[122,123]$ and from theoretical results [79].

Although not seen in the $\beta_{T}$ visualization of figure 5.31, the non-uniform pressure profiles of the circumferential stations show in figure 5.33 that at an early stage, at $x=-1.0$ and $x=-2.0$, there is already an influence of the stagger error distribution through the 

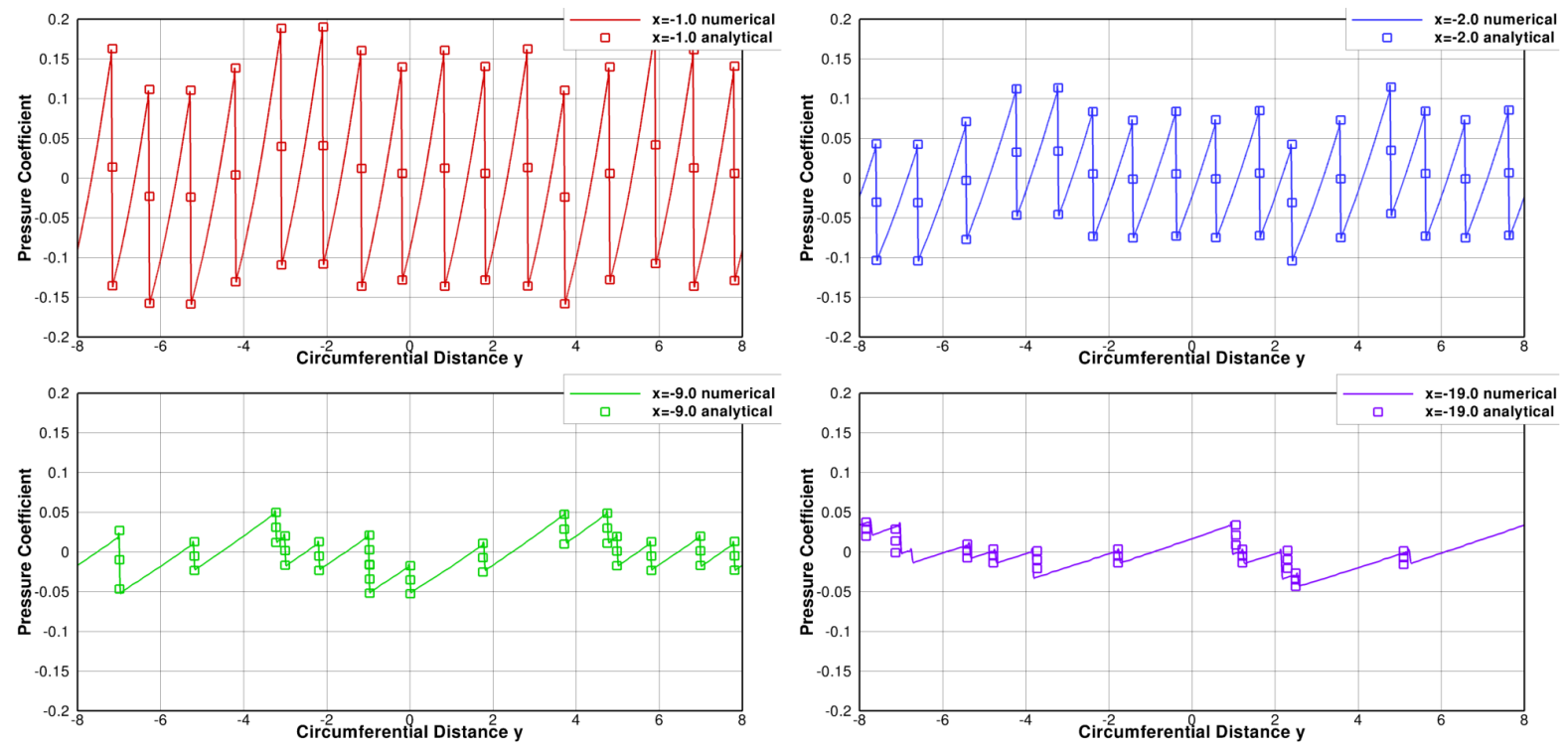

Figure 5.33: Numerical and theoretical pressure coefficient profiles of the circumferential stations for the supersonic realistic rotor blades cascade configuration.

rotor blades. For the other pressure coefficient circumferential stations, a reduction of the sawtooth waves amplitude is still observed similar to the previous cases. But as the axial distance increases at $x=-9.0$ and $x=-19.0$, due to different propagation velocities of different sawtooth profiles, the stronger shock-waves catches up and merges with the weaker ones, reducing the number of shock-waves. Comparing the numerical results with the shock-waves information provided by the analytical method, it is easy to see a strong concordance by these two methods. The initial condition of the analytical method is the information provided by the shock characteristics at $x=-1,0$ of the numerical results. The first result of the shock-wave propagation and comparison is shown at $x=-2.0$, where there is a noticeable amplitude decay of the shock-waves and the coalescence did not occur yet. At a typical rotor to nacelle lip distance at $x=-9.0$ the two methods still have strong concordance, where in a practical sense two shock-waves coalescence already have or are to occur at a very near axial position. Even though it is not in the equivalent nacelle region anymore, at the axial distance of $x=-19.0$, located at the end of the regular mesh region of the numerical simulation, the two methods still show strong coherence by only missing the exact coalescence of two shock-waves, validating with strong concordance the numerical method. 


\subsubsection{Supersonic Rotor and Stator Interaction in Realistic Conditions}

Now we consider the case with the most realistic configuration regarding supersonic rotor blades and stator vanes cascades in this work. The rotor blades cascade has the same stagger angle error on blade-to-blade distribution used in the previous rotor blades cascade MPT noise case. The stator vanes cascade has a perfectly positioned configuration; there is no stagger angle errors nor any stator-to-stator vanes difference. The first result of this configuration is the $\beta_{T}$ flow visualization shown in figure 5.34. The system of shock-waves produced by the supersonic passage of the rotor blades is very similar to the one presented in figure 5.31 of the previous case. The $\beta_{T}$ texture of the inlet region between the shock-waves is similar to the one found in figure 5.27 from the rotor and stator interaction case without the rotor cascade stagger angle errors. This is due to the interaction between the rotor and stator cascades. Even though the flow is primarily supersonic, there is a subsonic region between the rotor leading edge and the shock-wave where all the rotor and stator interaction propagates to the inlet region. Due to the shock-wave shielding these interactions cannot be properly assessed by the small perturbations linear theory and a relation between the shock-waves and the interaction waves, if possible, has yet to be developed.

The spectra of the static pressure probes located at the circumferential stations defined in figure 5.22 are shown in figure 5.35. The same shaft order tones development from the previous case can be observed, where no strong differences in the tones distribution can be seen. The rotor and stator cascade interaction noise observed in figure 5.34 is not strong enough to have any influence on the pressure probes spectra of all the axial positions.

The circumferential pressure coefficient distribution for various axial stations is shown in figure 5.36. The rotor-to-rotor blade stagger angle error effect can also be seen in the nearer pressure distribution at $x=-1.0$, the location of initial conditions for the analytical method. The next circumferential station at $x=-2.0$ shows a similar behavior as the MPT case without the stator vanes cascade. The analytical method agrees really well with the numerical results for the first two axial measurements. For the last two circumferential measurementes, at $x=-9.0$ and at $x=-19.0$, the behavior observed in the previous measurements positions is no more. The analytical method does not agree with the numerical results as in the rotor only MPT case. The rotor and stator interaction disturbances affect 


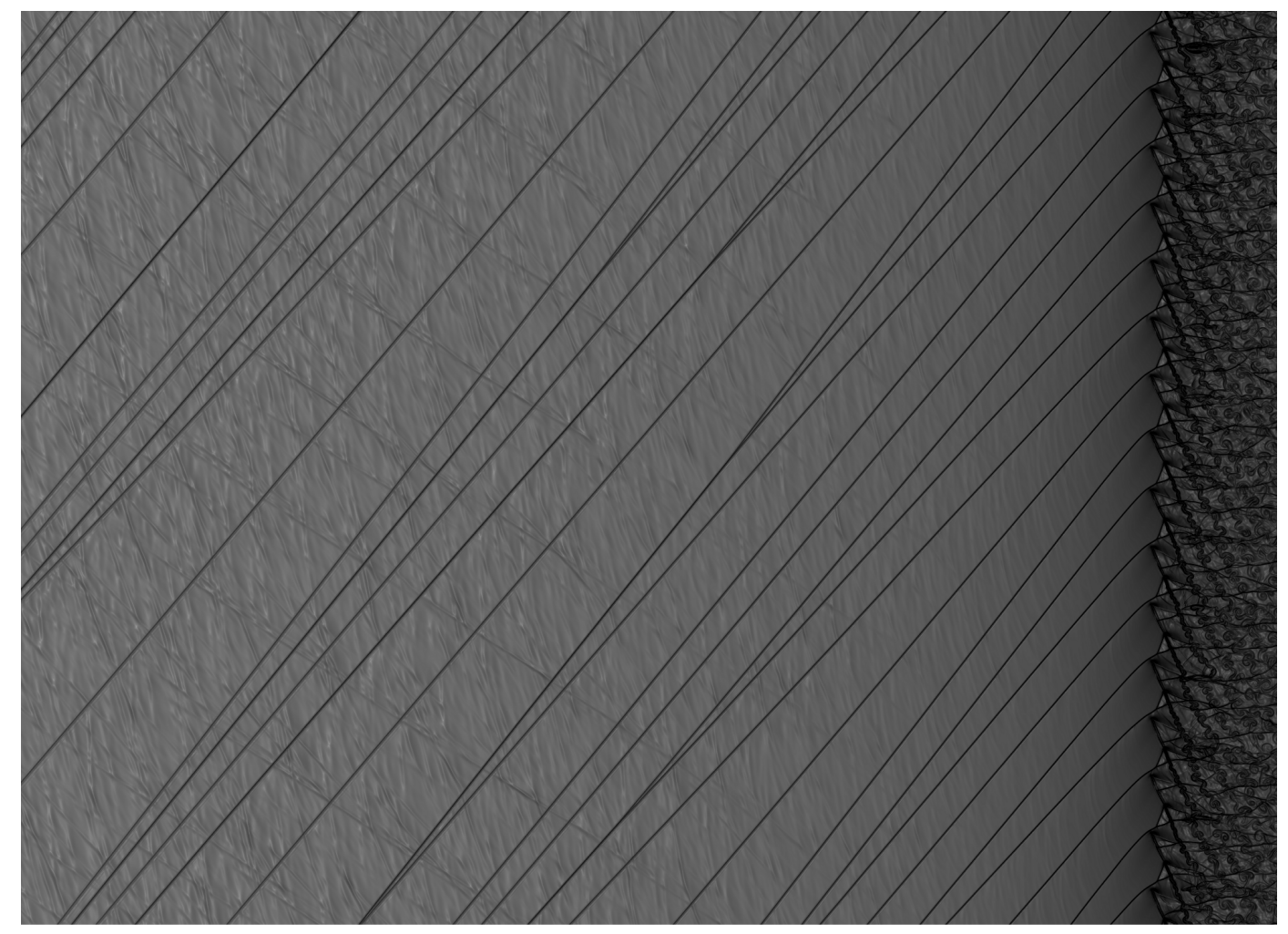

Figure 5.34: The $\beta_{T}$ visualization of the supersonic realistic rotor blades and stator vanes cascades configuration.

the expansion waves located between the propagating shock-waves. The assumption of linear shaped expansion waves cannot be considered for the last two circumferential stations and therefore the considerations made for the analytical method are not adequate anymore. The shock-waves decay and their propagation velocities cannot be exactly predicted but the pressure distribution and shock-waves signature can still be qualitatively represented by the analytical method.

\subsection{Expansional Viscosity Test in Rotor and Stator Su- personic Interaction}

The last section of results is about the merger of the two lines of research developed in this work. Even though it is at an incipient stage, the expansional viscosity model retains application possibilities that makes viable a first study of its effects according to 

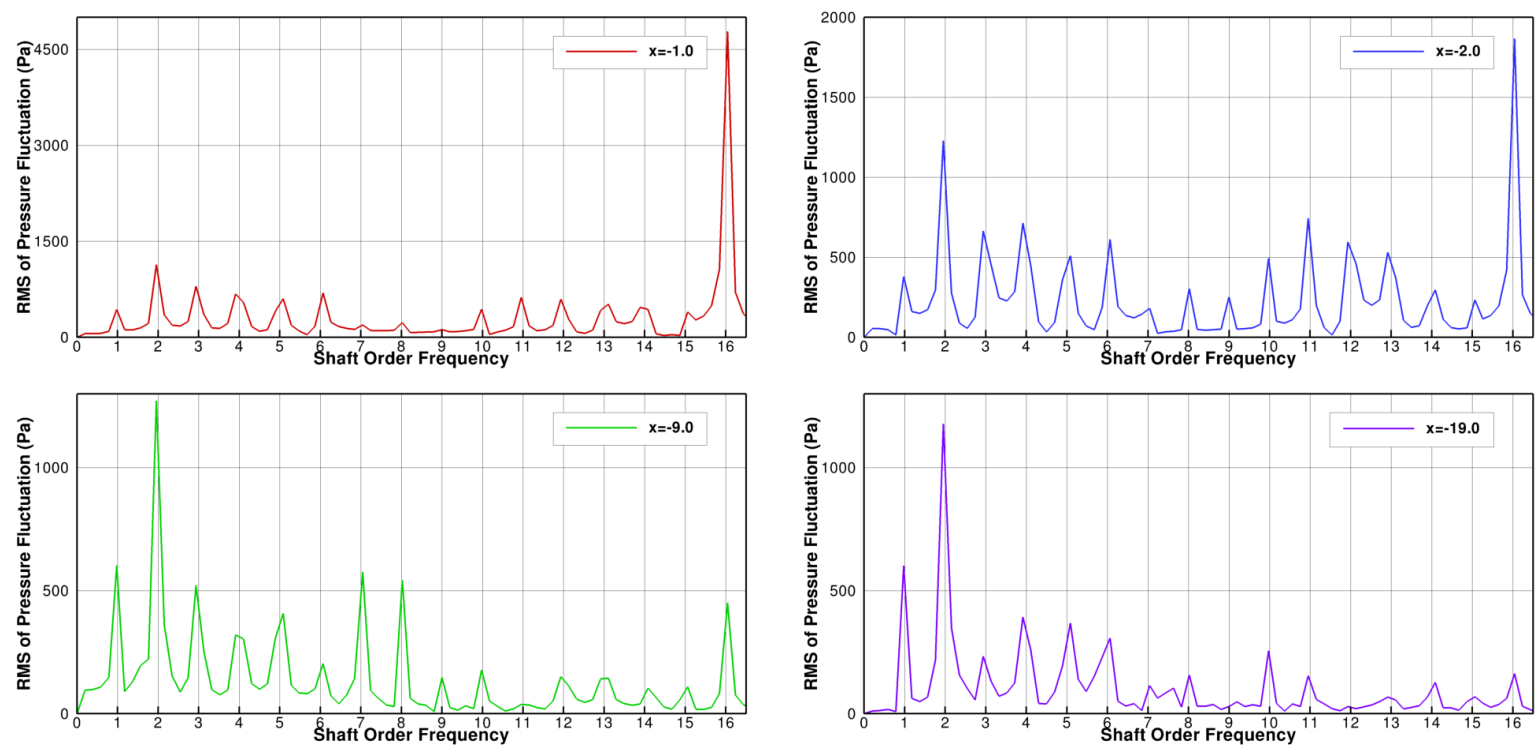

Figure 5.35: Spectra for the first harmonic of pressure probes from the supersonic rotor blades and stator vanes cascades case showing the multiple tone noise generation from shaft order 1 to 16, being the later equal to the blade passing frequency.
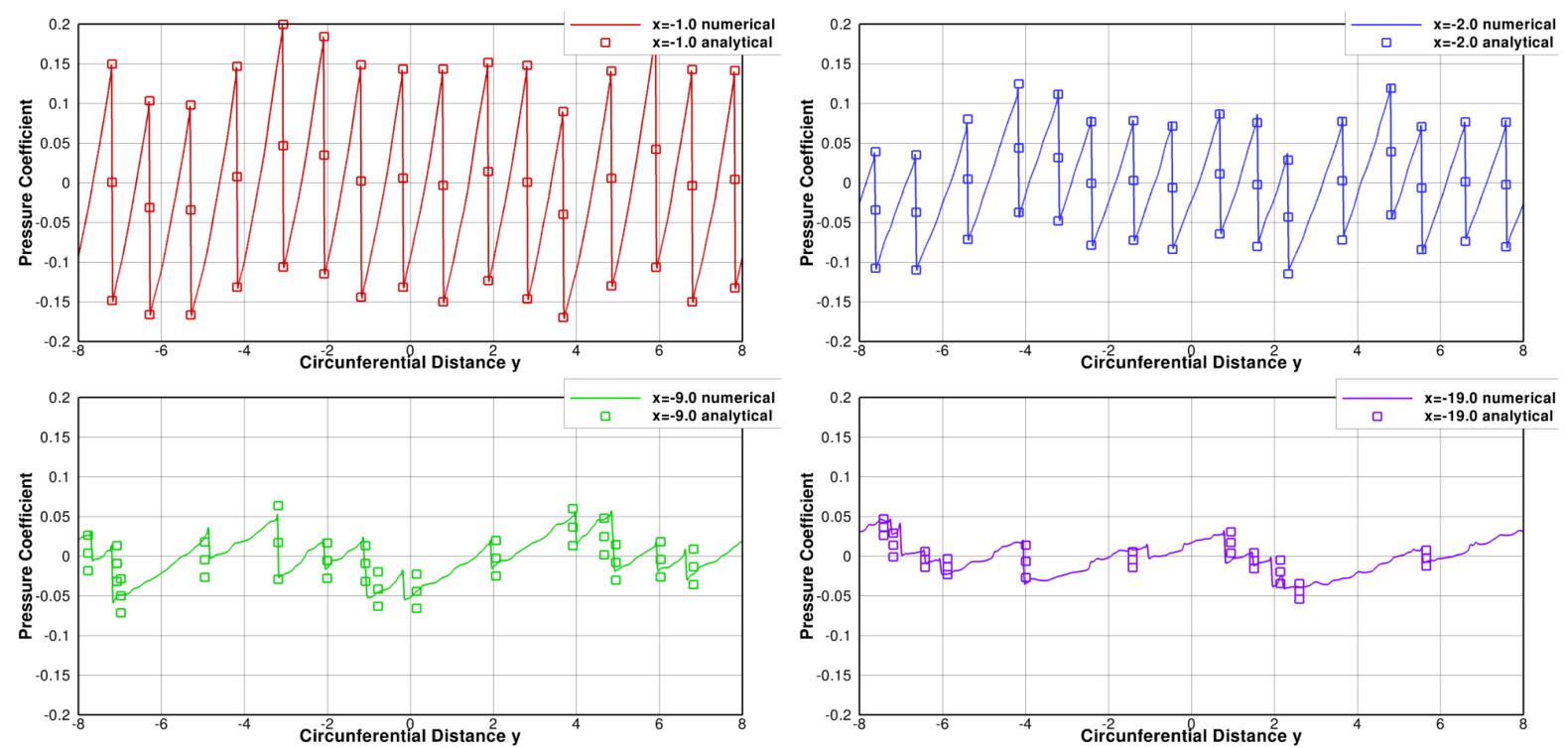

Figure 5.36: Pressure coefficient profiles of the circumferential stations for the supersonic realistic rotor blades and stator vanes cascades configuration.

the identified physical parameters. The chosen test case was the supersonic rotor and stator cascade interaction with the rotor cascades in nominal conditions. The shock-waves interactions on the cascade region and its later propagation in the inlet direction is a truly 
remarkable case for a first study of the expansional viscosity in realistic conditions.
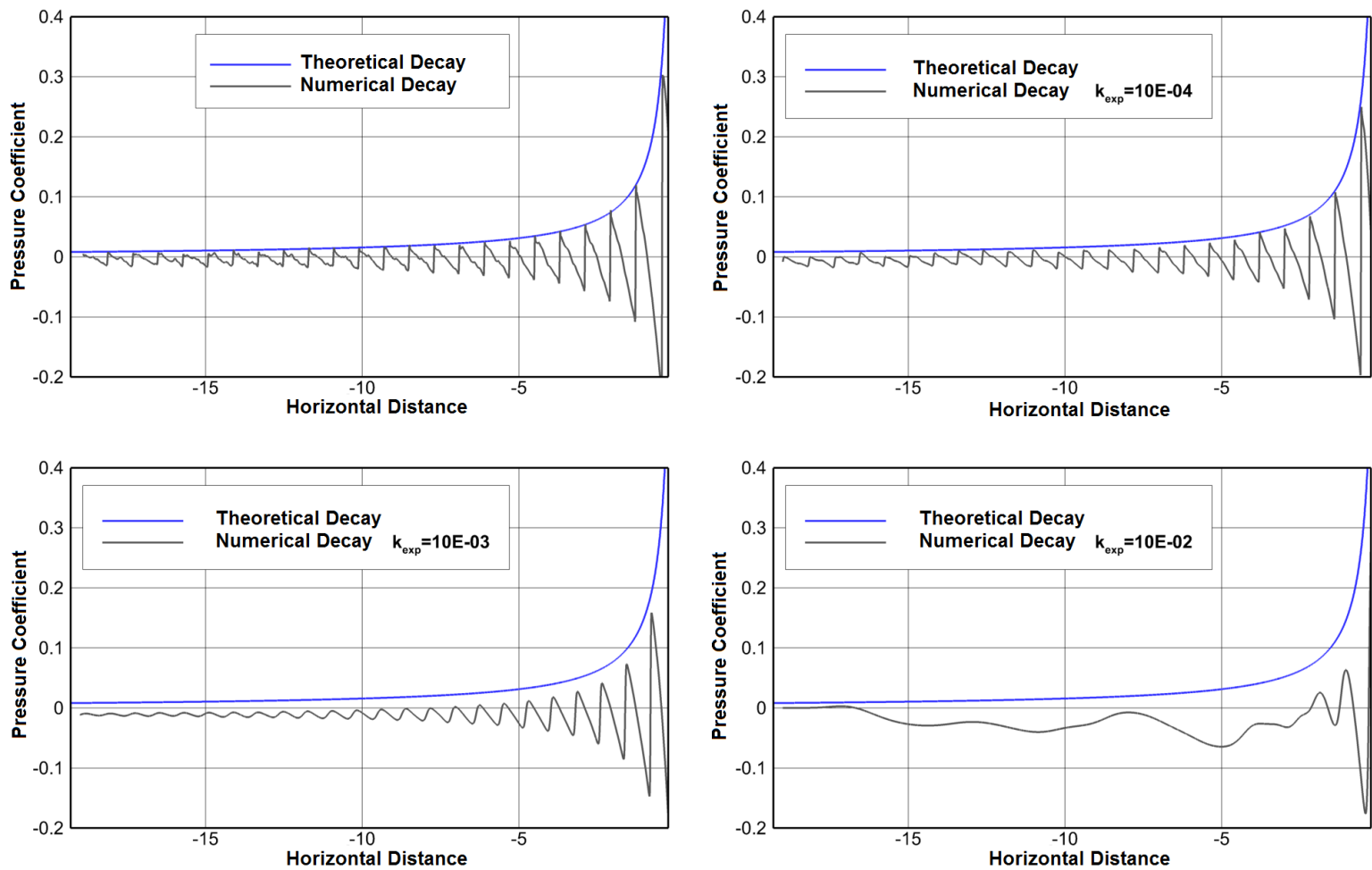

Figure 5.37: Comparison of the decay rate of the shock-waves intensity for the supersonic rotor and stator cascade interaction for several expansional viscosity parameter cases and a base case.

Identical operating conditions were used for the expansional viscosity cases. Three orders of magnitude of the parameter $k_{\exp }$ were simulated: $10^{-4}, 10^{-3}$ and $10^{-2}$. The result for comparison is the shockwave decay through the axial distance towards the flow inlet. The number of completed iterations in each case was approximately the same as the base case; one million of iterations after the acoustic perturbations reached the inlet region of the regular mesh.

Figure 5.37 shows the horizontal distribution of the pressure coefficient for all cases. The base case result is repeated here for convenience. In the first value of $k_{\exp }=10^{-4}$ the expansional viscosity effects are of little evidence, the phase distribution of the shock-waves was not yet modified, only some attenuation on its amplitude can be perceived. For the case with $k_{\exp }=10^{-3}$ the expansional viscosity influence becomes more apparent. The shock-waves amplitudes have even more attenuation and some dispersive effects can also 
be observed. For the fourth case, with $k_{\text {exp }}=10^{-2}$, the effects of the expansional viscosity becomes more aggressive, its influence affects all the shock-wave system. With a strong dispersion of the shock-waves, as seen in the realistic cases, the shock-waves coalescence occurs through all the regular computational domain. The pressure field does not follows anymore the decay trend presented by the theoretical analysis, the base case and the other cases with expansional viscosity.

Figure 5.38 shows the $\beta_{T}$ variable visualization of the base case and the cases with application of the expansional viscosity model. The figure is composed as follows: base case at the top left position, expansional viscosity case with $k_{\text {exp }}=10^{-4}$ at the top right position, $k_{\text {exp }}=10^{-3}$ at the inferior left position and $k_{\text {exp }}=10^{-2}$ at the inferior right position. In the first case of expansional viscosity (top right), there can be seen that visually the shockwaves distribution is almost the same as the base case, where the acoustic response due to the rotor and stator cascade interaction were somewhat attenuated. On the second case (inferior left), the shock-wave attenuation became more intense as they propagated towards the inlet region. The interaction response between the shock-waves was almost entirely eliminated in this case. On the last case (inferior right), as observed before on the axial distribution of the pressure coefficient (figure 5.37), the shock-wave attenuation was intense through all the propagation region. The shock-waves coalescence begins to take place very near the rotor blades cascade, where the regularity of the shock-waves among the rotor blades is totally affected, resulting in lesser waves at that region and a unique wave far from the rotor cascade.

Another result to be noted is directly related to the vorticity structures emitted by the trailing edge of the rotor blades that will interact with the stator vanes cascade, as shown in figure 5.39. The expansional viscosity did not have any effect on the vortex wake, only on the flow compressibility. Even though the vortex wake ordering was modified by the shockwaves interaction of varying strength, depending on the case, the characteristic length of these vorticity structures is the same for all four cases. 


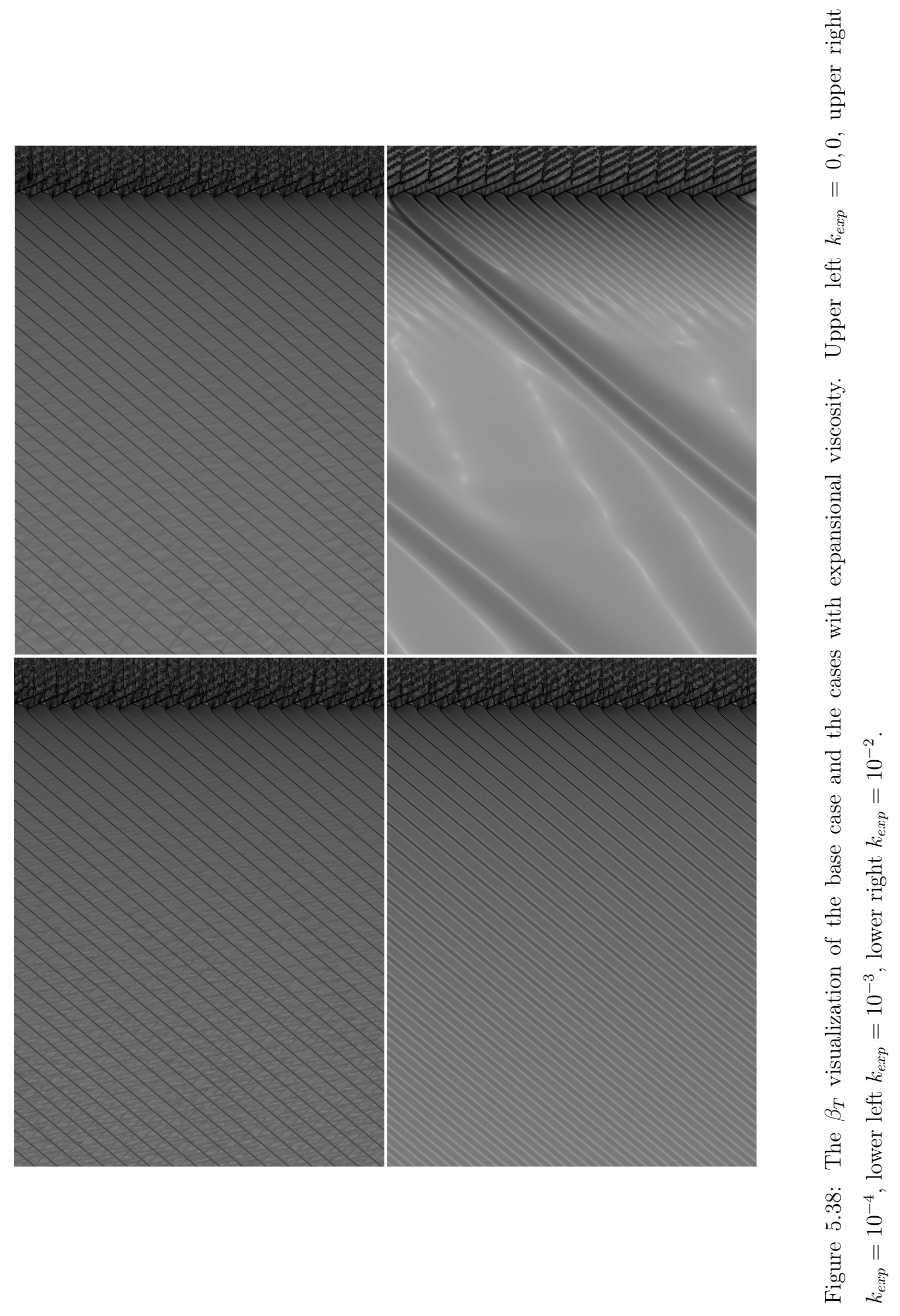



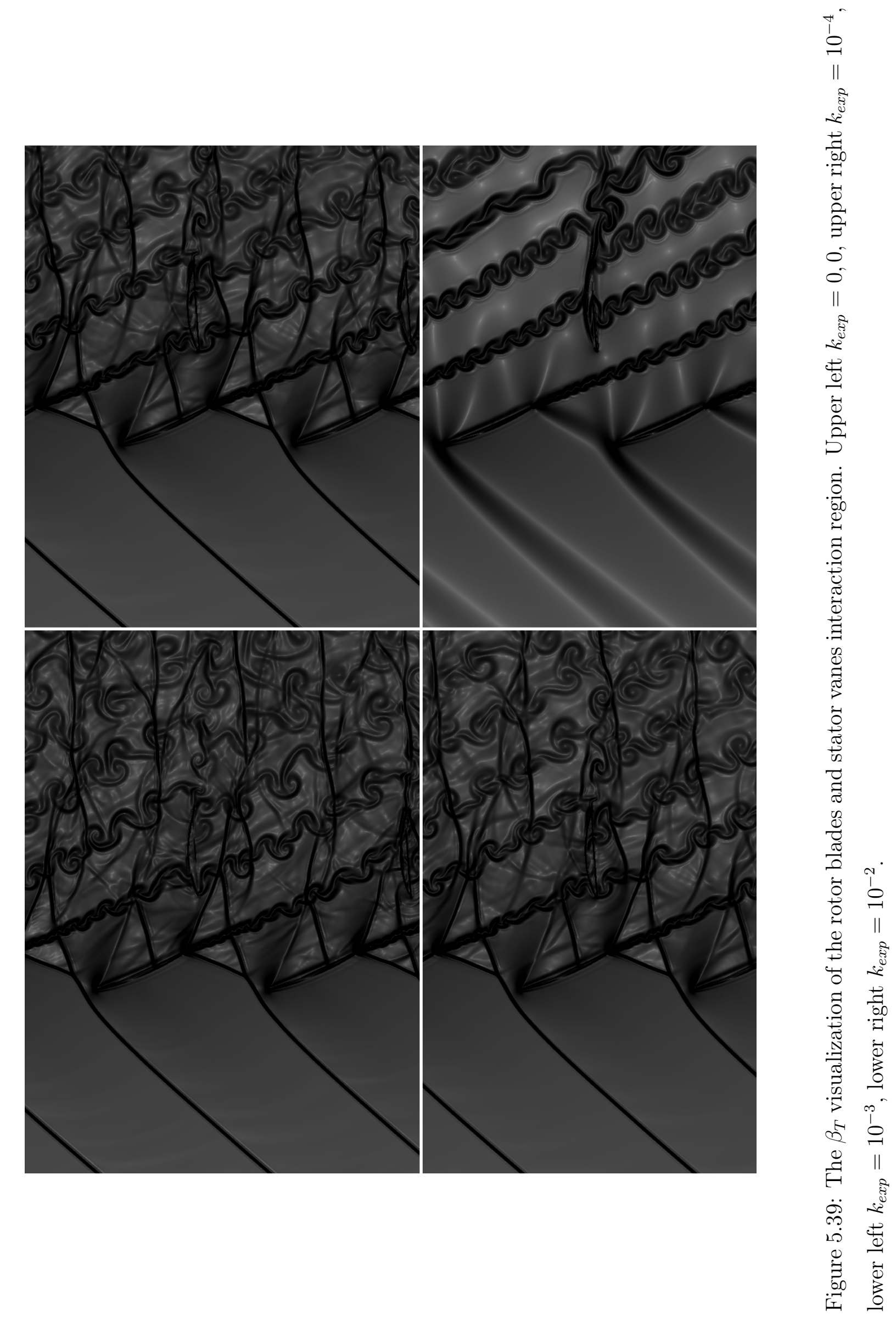


\section{Chapter 6}

\section{Conclusions and Future Work}

\subsection{Conclusions}

Modifications were proposed in almost all the numerical scheme of the moving immersed boundary applied to the finite volume formulation. The first modification was on the control volume value face numerical interpolation. The old interpolation scheme was proposed by Ducros, where a centered numerical stencil of four control volumes was used to interpolate the face value from the mean variables of these control volumes, resulting in an explicit interpolation. By using a spectral analysis of the Fourier transform from the space to the wavenumber domain, a general form of the centered interpolation was obtained. In implicit or explicit form, it was possible to predict over a linear range the behavior of the face reconstruction of the control volume for the Ducros scheme. It was found that at a certain wavenumber range, there was amplification of the signal when compared with the exact value. By defining the interpolation coefficients and using the Taylor series to formally obtain the maximum precision order with the same four control volumes centered numeric stencil, a non amplifying (in a spectral sense) interpolation scheme was proposed. A lower asymptotic behavior around the unity value of the transfer function of the exact value was obtained. Besides that, according with numerical considerations found in the literature, for the required definition of a good wave description for a second order numerical scheme, it was possible to predict the discretization error of a propagating wave for the formal fourth order proposed scheme. This error was at least one order of magnitude smaller 
when compared with the second order numerical base scheme, justifying the use of the proposed numerical scheme and the computational mesh of the rotor and stator cascade interaction cases of this work. The core of the immersed boundary method relies upon direct imposition of the boundary conditions on the control volumes in computational cases that present highly numerically unstable flow elements (steep physical gradients). The immersed boundary method needs stabilization schemes that makes the numerical solution stable without affecting the physical properties of the flow. Initially the artificial viscosity numerical scheme used on VAT was not strong enough to make the code stable in the studied cases of this work. From the artificial viscosity scheme previously used on VAT, new numerical dissipation sensors based on kinematic and thermodynamic variables were proposed. These were based on the flow vorticity, divergent, pressure, and density gradients. The four proposed new numerical dissipation sensors were able to make the code numerically stable in all the studied cases without affecting the physical properties of the flow. The last proposed modification was the implementation of a slipping boundary condition of the tangential velocity component on the body control volumes. The slipping boundary condition was used because of the nature of the governing equations (Euler equations), where by the lack of fluid viscosity results in no boundary layer on the body surface, or its thickness is infinitely small. This proposed boundary condition was used with the other ones for the other flow variables and no further modifications were necessary, as a result for the invariant properties of these boundary conditions for inertial frames of reference.

Superb performance gains were obtained for the VAT code with the CUDA architecture implementation. By using certain implementation and execution strategies of the numerical scheme in CUDA Fortran, it was possible to achieve a speedup of around one hundred times when compared with a serial code execution on a common CPU core. The conservative variables declaration as permanent on the global GPU memory during the code execution enabled the complete offloading of the numerical computation from the CPU to the GPU without loss of performance due to the small PCI-express bus communication bandwidth, which would be inevitable at each executed iteration. The communication between the GPU and its node was reduced to the strictly necessary ones, like the data reading and output and the process communication at a distributed memory level (MPI processes). The numerical subroutine that took most of the computational resources was the Euler 
equations associated numerical solver. Most of the optimization efforts for performance gain were spent in this subroutine, where roughly $90 \%$ of the total iteration time is spent. The decisions for optimization were taken almost fully at a user level, restricting the automatic compiler optimization. In the individual grid block processing, where each block is processed separately by a SM of the GPU, the working variables declaration was done at the chipset memory level, which has an extremely low latency but small capacity and same duration as the block processing. The use of this memory was both as registers (thread restricted scalars) and as shared memory (thread block common arrays). For read only arrays, like the mesh discretization constants, such as face values and volume inverses, the texture memory cache was used for a fast access of these values on the GPU global memory. From these two ideas of optimization in the code, it was possible to obtain extremely high performance gains on the main code subroutine. The other subroutines of the code were written in a way to let the compiler control the optimization process, where parallel directives very similar to the OpenMP for execution loops were used on the GPU variables working regions. Being simpler algorithms, the performance gain by compiler controlled optimization was satisfactory on these subroutines. The main strategy to avoid the PCI-express bottleneck brought some limitations to the code execution; the permanent variable declaration only on the GPU global memory sets a limit on the problem size to be simulated. Fortunately with the increase of the GPU global memory size with each new generation, this problem will be mitigated as the GPUs' total memory tends to be of the same size as the RAM memory of the same computing node. CUDA architecture proved to be an extremely useful and effective scientific and engineering high performance computing tool, as an excellent alternative for CPU execution only codes. The CUDA architecture also proved to be mature enough for high performance computing applications where a tradeoff must be paid for adapting or even rewriting whole numerical codes with the needed language extensions for the GPU computation offload.

The rotor blades and stator vanes cascade interaction were studied by using the moving immersed boundary method for all the flow compressibility regimes found in a typical turbofan engine: subsonic, transonic and supersonic. The thin duct approximation was used to make a more viable the comparison between the analytical and numerical results. On the subsonic and transonic cases the theory regarding the acoustic modes generated by cascade 
interaction and propagated through the annular duct agreed very well with the results from the numerical simulations. Excellent agreement was observed with the angle measurement of the dominant modes that could be identified in the aeroacoustic visualization. The modal decomposition by means of a spectral analysis of the moving pressure probes has also shown excellent agreement with the analytical results. All the predicted modes could be identified by the modal decomposition up to the fourth harmonic of the blade passing frequency. The supersonic rotor and stator interaction case needed a different treatment for the validation process. The first validation was with the generated shock-waves behavior and its propagation towards the inlet direction for the rotor blades cascade only. Outstanding agreement was observed for the amplitude decay of these shock-waves. The stator cascade influence on the supersonic rotor blades cascade case was also assessed. The rotor and stator interaction was not enough to produce any change in the shock-waves decay, even though the expansion waves between the shocks had some modification due to the cascade interaction similar to what was found in the subsonic and transonic cases. The third case with supersonic rotor cascade was with slight geometry modifications of the rotor blades to produce the multiple pure tone noise. A randomly generated distribution of fixed increments of the stagger angle was used to produce the flow effects that induced the buzz-saw noise. By comparing the shock-waves propagating to the inlet direction with the results from the theoretical values for the decay and shock coalescence, excellent agreement was observed in all the considered circumferential positions, including the equivalent nacelle lip position and at the end of the regular computational control volumes mesh. The pressure probes spectral analysis at several positions in the inlet region revealed the typical energy transfer from the higher shaft frequency harmonics to the lower ones, a phenomenon already observed in experiments that produce the well known buzz-saw noise signature. The fourth supersonic rotor case was the most complete one, with the stator cascade and random stagger angle modification of the rotor blades. Similar results were obtained when compared to the rotor cascade alone in realistic conditions on the pressure probes spectral analysis. The pressure field obtained numerically and the analytical results did not have the same excellent agreement as in the rotor only case. This was due to the cascade interaction propagating between the shock-waves and then affecting in an disorderly way the expansion waves between them. This modification of the expansion waves affected the shock-waves decay, propagation and consequently the coalescence. The interaction influence on the expansion 
waves was not considered for the analytical theory, where a perfectly linear expansion wave is considered, resulting in an uneven decay between the high and low pressure parts of the shock-waves. Though the results differ in the shock-wave signature, they qualitatively agree to some extent.

The expansional viscosity model reported by Landau was extensively studied along with its application to a non-Stokesian fluid, where the thermodynamic non-equilibrium state is hypothetically considered in flows with regions of rapid compression and expansion. The expansional viscosity model was applied in the stress tensor for a fluid and its effects were identified, directly related to the dissipation term, as a function of the divergence of the velocity field and the fluid density. A linear stability analysis was carried as a first study of the expansional viscosity behavior, in a fluid with no shearing viscosity. The governing equations used in this analysis were the continuity equation, the Cauchy equation with the expansional viscosity model applied to the stress tensor and the barotropic equation for the pressure and density closure. The case studied on the stability analysis was the decay time decay of a one dimensional cyclic wave in the frequency domain. The non dimensional physical parameters that are directly related to the case of wave propagation were identified and its sensitivity to wave number was assessed. In all cases of the physical parameters analysis, the amplifying factor was only decay of the wave amplitude. No cases of wave amplification were found for all the wave number spectrum analyzed. For a Stokesian inviscid fluid, no amplification nor decay of the wave was observed, only the transport of its related quantities (mass and momentum). Two solutions were obtained for the amplification factor in the stability analysis. The observed behavior of these solutions was asymptotic values or monotonic increase of the wave decay for higher values of wave number. The linear stability analysis has shown that, at least for the linear range, the expansional viscosity model is physically coherent, acting only as of diffusive character for all the range of the tested physical parameters. In the nonlinear regime (not taken into account in the linear stability analysis) a numerical scheme with very high formal and spectral precision of finite differences for the space discretization and explicit Runge-Kutta time marching was proposed. The stability of the method was achieved by using a high frequency filtering after a given number of iterations was complete. Starting from the same formulation used in the linear stability analysis, the inverse Fourier transform was applied 
to the expansional viscosity model and a nonlinear evolution model in the time domain was obtained and proposed for numerical study. Comparing the corresponding physical parameters between the time domain from the numerical simulation, and spectral domain from the stability analysis, it was possible to identify the linear regime range as a function of the wave velocity amplitude. The linear stability analysis is not explicitly dependent on this wave amplitude. The same amplification factor is found regardless of the wave amplitude. The value of the amplification factor is the solution from the stability analysis. In the numerical simulations the amplification factor was obtained with an exponential regression from the wave velocity amplitude solution through time, and then compared with the theoretical values. For velocity wave amplitudes lower than $10^{-3}$, the numerical amplification factor has excellent agreement with the theoretically obtained results. For higher values, the flow regime begins to diverge from the linear until a seemingly exponential divergence of the amplification factor as a function of the wave speed amplitude is seen. This is due to the accumulation of momentum at the wave crest and trough as a result of the different propagation speeds along the wave. As a consequence, steeper gradients will result in larger differences in propagation velocities along the wave and therefore its nonlinear effects, thus explaining the amplification factor reduction with high values for the wave velocity amplitude in the numerical simulations. The physical parameters analysis at the theoretical and numerical sides has shown important aspects of the expansional viscosity model, like the proportion of these parameters which affects the wave decay as a function of its wave number, i.e., the wave frequency. Regarding the values of the wave velocity amplitudes in the nonlinear region for different orders of magnitude for the wave number, the time needed to achieve the same wave amplitude, from the case with the wave number one order of magnitude smaller, was one hundred times smaller, showing a logarithmic decay as the frequency of the wave rises. Another important result from the numerical simulation was the response of the expansional viscosity coefficient for several values of the related physical parameter $\Psi$. Depending on the value of this parameter, the wave maximum value of the expansional viscosity coefficient may oscillate and be decayed until a final value is reached.

The last result of this work is about the expansional viscosity model application in a realistic case of numerical simulation. The expansional viscosity model with instantaneous 
response was implemented into the VAT numerical code. The chosen case for comparison of the expansional viscosity effects, for several values of the correspondent physical parameter, was the supersonic rotor cascade in nominal conditions interacting with the stator vanes cascade. Three values differing in order of magnitude for the expansional viscosity parameter were simulated and then compared with the base case with no expansional viscosity at all. The shock-wave decay in the cases with this model was more intense with higher values of the associated physical parameter, where in the case with the highest value the shock-wave spreading was so intense that there was the coalescence between all the shockwave system at a given horizontal position. The last result that was observed was about the vorticity wake from the rotor blades trailing edge. Even though a byproduct of compressibility effects, the vorticity structures were not affected by the expansional viscosity acting on the flow, keeping its characteristic length.

\subsection{Future Work}

Although all the objectives of this work were completed, further development is needed for all the methodologies and models used here. Of course the numerical study of the moving immersed boundary still need more validation beyond from the two dimensional cases. Validation with three dimensional cases and comparison with experimental results is likely the next step towards the VAT code being able to be used by industry for routine capacity cases. The radial modes would be taken into account in the code validation for the sound propagation in ducts, where all the flow regimes would be considered as well (subsonic, transonic and supersonic). Physics enhancement used in the VAT code must also be taken into account: turbulence models and wall functions to compensate the inherent lack of resolution at the wall is a needed feature. Since out of the duct the sound waves behave linearly for most flow regimes, propagation and radiation techniques could be used for sound propagation into the farfield, including the use of some acoustic analogies similar to what was presented in appendix II. A multiblock discretization scheme would be useful to reduce the control volume count in the physical domain, so that a huge control volume density that was used in the noise source region would not be needed for acoustic propagation and radiation in more well behaved flow regions. The expansional viscosity model has 
shown great potential to be used for really long propagation distances calculation and in regions of high compression and expansion in fluids. An experimental validation of the model, derivation of parameter closure relations and their calibration would be the next step for making it more suitable for physical studies. A second order model could be even more precise than the first order model used here. Also two and three dimensional tests with simple propagation problems could be done to understand better the effects of the expansional viscosity. 


\section{Bibliography}

[1] Rey, E., Effect of Nocturnal Aircraft Noise on Psychological Stress Indicators, DLRForschungsbericht 2002-14, 2002, 325 Seiten, 24 Bilder, 90 Tabellen, 240 Literaturstellen

[2] Kiernan, V., Noise Pollution Robs Kids of Language Skills, New Scientist, 10 May 1997, p. 5

[3] Bond, M., Plagued by Noise, New Scientist, 16 November 1996, p. 14-15

[4] International Civil Aviation Organization, Environmental Technical Manual Volume I: Procedures for the Noise Certification of Aircraft, DOC-9501

[5] http://www.icao.int/Meetings/EnvironmentalWorkshops/ Documents/Noise-Certification-Workshop-2006/Depitre_4.pdf

[6] Smith, M. J. T., Aircraft Noise, Cambridge University Press, Cambridge, United Kingdom, 1989

[7] http://www.aeronautics.nasa.gov/docs/chicago/noise.htm

[8] http://www.dlr.de/as/en/desktopdefault.aspx/tabid-192/402_read-1633/

[9] Manoha, E., ONERA's Activities on Airframe Noise numerical Simulation, J. Acoust. Soc. Am. Volume 123, Issue 5, pp. 3392-3392 (2008)

[10] http://www.aero.jaxa.jp/eng/research/aircraftsys/airtech.html

[11] http://wwW.grc.nasa.gov/WWW/Acoustics/media/200405. Manchester.pdf 
[12] Berton, J., Envia, E., and Burley, C., An Analytical Assessment of NASA's $N+1$ Subsonic Fixed Wing Project Noise Goal, AIAA Paper 2009-3144, 15th AIAA/CEAS Aeroacoustics Conference, Miami, FL, 11-13 May, 2009

[13] http://www.nasa.gov/topics/aeronautics/features/aircraft_noise.html

[14] https://mdao.grc.nasa.gov/topstoryarchive015.html

[15] http://www.purepowerengine.com/pdf/PW1700G-1900G_05_13\%28F\%29.pdf

[16] http://www.aviationweek.com/Article.aspx?id=/article-xml/ awx_01_08_2013_p0-534517.xml

[17] http://ntrs.nasa.gov/archive/nasa/casi.ntrs.nasa.gov/ 20080006600_2008004016.pdf

[18] Batchelor, G. K., An Introduction to Fluid Dynamics, Cambridge Mathematical Library, First Edition, Cambridge University Press, 2010

[19] Vincenti, Walter G., Kruger Jr., Charles H., Introduction to Physical Gas Dynamics, Krieger Publishing Company, Malabar, Florida, 1986

[20] Landau and Lifshitz, Course of Theoretical Physics, Fluid Mechanics, Elsevier, 2nd edition, volume 6, 2012

[21] Emanuel, George, Bulk Viscosity of a Dilute Polyatomic Gas, Phys. Fluids A 2 (12), December, 1990

[22] Hoover, William G., Ladd, Anthony J. C., Hickman, Richard B., Holian, Brad Lee, Bulk Viscosity via Nonequilibrium and Equilibrium Molecular Dynamics, Physical Review A, Vol. 21, No. 5, May, 1980

[23] Hirsch, C., Numerical Computation of Internal and External Flows, Elsevier, 2nd edition, 2007

[24] Slotnick et al., CFD Vision 2030 Study: A Path to Revolutionary Computational Aerosciences, NASA/CR-2014-218178 
[25] Tam, Christopher K. W., Computational Aeroacoustics, a Wave Number Approach, Cambridge University Press, 2012

[26] http://www.mooreslaw.org/

[27] https://developer.nvidia.com/cuda-zone

[28] https://www.khronos.org/opencl/

[29] http://www. openacc-standard.org/

[30] http://www.pgroup.com/resources/cudafortran.htm

[31] http://www. mathworks.com/products/matlab/

[32] https://software.intel.com/en-us/mic-developer

[33] http://www.ansys.com/Products/Simulation+Technology/Fluid+Dynamics/ Fluid+Dynamics+Products/ANSYS+Fluent

[34] http://www.ansys.com/Products/Simulation+Technology/Fluid+Dynamics/ Fluid+Dynamics+Products/ANSYS+CFX

[35] http://www. metacomptech.com/index.php/products/cfd

[36] http://www.exa.com/powerflow.html

[37] http://www.top500.org/lists/2014/06/

[38] Maunus, J., Grace, Sheryl M., Sondak, Douglas 1., Effect of Rotor Wake Structure on Fan Interaction Tone Noise, AIAA Journal, Vol. 50, No 4, April 2012

[39] Polacsek, C., Burguburu, S., Redonnet, S., Terracol, M., Numerical Simulation of Fan Interaction Noise Using a Hybrid Approach, AIAA Journal, Vol. 44, No. 6, June 2006

[40] Sharma, Anupam, Richards, Simon K., Wood, Trevor H., Shieh, Chingwei, Numerical Prediction of Exhaust Fan Tone Noise from High Bypass Aicraft Engines, 13th AIAA/CEAS Aeroacoustics Conference, AIAA 2007-3700, 2007 
[41] Weckmüller, Christian, Guérin, Sébastien, Ashcroft, Graham, CFD-CAA Coupling Applied to DLR UHBR-Fan: Comparison to Experimental Data, 15th AIAA/CEAS Aeroacoustics Conference, AIAA-2009-3342, Miami, Florida, USA, 2009

[42] Grace, Sheryl M., Sondak, Douglas L., Eversman, Walter, Cannamela, Michael J., Hybrid Prediction of Fan Tonal Noise, 14th AIAA/CEAS Aeroacoustics Conference, AIAA-2008-2992, Vancouver, British Columbia, Canada, 2008

[43] Hase, Takaaki, Yamasaki, Nobuhiko, Ooishi, Tsutomu, Numerical Simulation for Fan Broadband Noise Prediction, Journal of Thermal Sciences, Vol.20, No.1, 2011

[44] Hu, Bin-bin, OuYang, Hua, Wu, Ya-dong, Jin, Guang-yuan, Qiang, Xiao-qing, Du Zhao-hui, Numerical Prediction of the Interaction Noise Radiated from an Axial Fan, Applied Acoustics, Vol.74, pp.544-552, 2013

[45] Kazawa, Junichi, Horiguchi, Yasuo, Saiki, Kazuhisa, Yamamoto, Kazuomi, Nozaki, Osamu, Oishi, Tsutomu, Numerical Study on Fan Noise Generated by Rotor-Stator Interaction, 13th AIAA/CEAS Aeroacosutics Conference, AIAA-2007-3681, 2007

[46] Li, Qinling, Peake, Nigel, Savill, Mark, Large Eddy Simulations for Fan-OGV Broadband Noise Prediction, 14th AIAA/CEAS Aeroacoustics Conference, AIAA-20082992, Vancouver, British Columbia, Canada, 2008

[47] Verdon, Joseph M., Linearized Unsteady Aerodynamic Analysis of the Acoustic Response to Wake/Blade-Row Interaction, NASA/CR-2001-210713, 2001

[48] Dunn, M. H., Tweed, J., Farassat, F., The Application of a Boundary Integral Equation Method to the Prediction of Ducted Fan Engine Noise, Journal of Sound and Vibration, Vol.227(5), pp.1019-1048, 1999

[49] Gabard, G., Astley, R. J., A Computational Mode-Matching Approach for Sound Propagation in Three-Dimensional Ducts With Flow, Journal of Sound and Vibration, Vol.315, pp.1103-1124, 2008

[50] Verdon, Joseph M., Montgomery, Matthew D., Chuang, H. Andrew, Development of a Linearized Unsteady Euler Analysis With Application to Wake/Blade-Row Interactions, NASA/CR-1999-208879, 1999 
[51] Rienstra, S. W., Acoustic Radiation from a Semi-Infinite Annular Duct in a Uniform Subsonic Mean Flow, Journal of Sound and Vibration, Vol.94(2), pp.267-288, 1984

[52] Ffowcs Williams, J., E., Hawkings, D. L. Sound Generation by Turbulence and Surfaces in Arbitrary Motion, Philosophical Transactions of the Royal Society of London. Series A, Vol. 264, No. 1151 (May 8, 1969), pp. 321-342

[53] Ghorbaniasl, Ghader, Hirsch, Charles, Validation and Application of a Far-Field Time Domain Formulation for Fan Noise Prediction, 11th AIAA/CEAS Aeroacosutics Conference, Monterey, California, USA, 2005

[54] Hawkings, D. , Transonic Fan Noise, Her Majesty's Stationery Office, C.P. No. 1226, 1972

[55] Gliebe, P., Mani, R., Shin, H., Mitchell, B., Ashford, G., Salamah, S., Connell, S., Aeroacoustic Prediction Codes, NASA CR-2000-210244, NASA Glenn Research Center, 2000

[56] Paliath, U., Han, F., Shieh, C., Sharma, A., Multiple pure tone noise predictions for acoustically treated aircraft engines, 16th AIAA/CEAS Aeroacoustics Conference, AIAA 2010-3714, , Stockholm, Sweden, 2010

[57] Uellenberg, S., Buzzsaw noise predictions for modern turbofans, AIAA 2004-3000, 10th AIAA/CEAS Aeroacoustics Conference, Manchester, UK, 1012

[58] Fisher, M. J., Tester, B. J., Schwaller, P. J. G., Supersonic fan tone noise prediction, 4th AIAA/CEAS Aeroacoustics Conference, AIAA 98-2249, Toulouse, France, 1998

[59] McAlpine, A., Schwaller, P. J. G., Fisher, M. J., Tester, B. J., Buzz-saw noise: Prediction of the rotor-alone pressure field, Journal of Sound and Vibration, Vol. 331, pp.4901-4918, 2012

[60] Adetifa, O., McAlpine, A., Gabard, G., Modelling of the nonlinear sound propagation and radiation of supersonic fan tones, 20th AIAA/CEAS Aeroacoustics Conference, AIAA 2014-2945, Atlanta, United States, 2014

[61] Aris, Rutherford, Vectors, Tensors and the Basic Equations of Fluid Mechanics, First Edition, Dover Publications, 1989 
[62] Butkov, Eugene, Física Matemática, Livros Técnicos e Científicos Editora S.A., 1988

[63] Rienstra, S. W., Hirschberg, A., An Introduction to Acoustics, Eindhoven University of Technology, 2006

[64] Howe, M., S., Theory of Vortex Sound, Cambridge Texts in Applied Mathematics, Cambridge University Press, 2003

[65] Müller Gerhard, Möser, Michael, Handbook of Engineering Acoustics, Springer-Verlag Berlin Heidelberg, pp. 494-496, 2013

[66] Michel, U., Siller, H., DLR Lecture Script-Aircraft Noise Course, DLR IB 9251709/B2, November 17, 2010

[67] Goldstein, Marvin E., Aeroacoustics, Scientific and Technical Information Office, Washington, D. C., NASA, 1974

[68] Hubbard, H. H., Aeroacoustics of Flight Vehicles: Theory and Practice, Volume 1: Noise Sources, RP 1258, NASA Langley Research Center, Hampton, Viginia, 1991

[69] Tyler, J. M., Sofrin, T. G., Axial Flow Noise Compressor Studies, SAE Technical Paper 620532, 1962

[70] Rice, E. J., Heidmann, M. F., Sofrin, T. G., Modal Propagation Angles in a Cylindrical Duct With Flow and Their Relation to Sound Radiation, NASA Technical Memorandum 79030, January, 1979

[71] Envia, E., Benchmark Solution for the Category 3-Problem 2: Cascade-Gust Interaction, Fourth Computational Aeroacoustics (CAA) Workshop on Benchmark Problems, NASA/CP-2004-212954.

[72] Morfey, C. L., Fisher, M. J., Shock-Wave Radiation from a Supersonic Ducted Rotor, The Aeronautical Journal of the Royal Aeronautic Society, Vol. 74, pp. 579-585, 1970

[73] Cicon, D. E., Sofrin, T. G., Mathews, D. C., Investigation of Continuously Traversing Microphone System for Mode Measurement, NASA Report CR-168040, November, 1982 
[74] Sutliff, Daniel L., Rotating Rake Turbofan Duct Mode Measurement System, NASA Technical Memorandum 2005-213828, October, 2005

[75] Hawkings, D., Multiple Tone Generation by Transonic Compressors, Journal of Sound and Vibration, Vol. 17, pp. 241-250, 1971

[76] Morfey, C., Fisher, M., Shock-wave Radiation From a Supersonic Ducted Rotor, The Aeronautical Journal of the Royal Aeronautical Society, Vol. 74, pp. 579-585, 1970

[77] Pickett, G., Prediction of the Spectral Content of Combination Tone Noise, Journal of Aricraft, Vol. 9, pp. 658-663, 1972

[78] Philpot, M., The Buzz-saw Noise Generated by a High Duty Transonic Compressor, ASME paper No. 70-GT-57, 1970

[79] Kurosaka, M., A Note on Multiple Pure Tone Noise, Journal of Sound and Vibration, Vol. 19, pp. 453-462, 1971

[80] Sobral, Yuri Dumaresq, Estabilidade Hidrodinâmica e Magnética de Leitos Fluidizados, Dissertação de Mestrado, Departamento de Engenharia Mecância, Universidade de Brasília, 2004

[81] Lele, S., Compact Finite Difference Schemes with Spectral-like Resolution, Journal of Computational Physics, 103, 16-42, 1992.

[82] Kennedy, C. A., Carpenter, M. H., Lewis, R. M., Low-Storage, Explicit Runge-Kutta Schemes for the Compressible Navier-Stokes Equations, Applied Numerical Mathematics 35, 2000, pp. 177-219

[83] Logan, J. David, Applied Mathematics, John Wiley \& Sons, third edition, 2006

[84] http://www.netlib.org/lapack/

[85] http://www.netlib.org/blas/

[86] Peskin, C. S., Flow patterns around heart valves: a numerical method, Journal of Computational Physics, Vol. 10, 1972, pp. 252-271. 
[87] de Tullio, M. D., De Palma, P., Iaccarino, G., Pascazio, G., Napolitano, M., An immersed boundary method for compressible flows using local grid refinement, Journal of Computational Physics, Vol. 225, 2007, pp. 2098-2117.

[88] Cho, Y., Boluriaan, S., Morris, P. J. Immersed Boundary Method for Voiscous Flow Around Moving Bodies, 44th AIAA Aerospace Sciences Meeting and Exhibit, AIAA 2006-1089, 2006.

[89] Liu, Q., Vasilyev, O. V., A Brinkman penalization method for compressible flows in complex geometries, Journal of Computational Physics, Vol. 227, 2007, pp. 946-966.

[90] Ghias, R., Mittal, R., Dong, H., A sharp interface immersed boundary method for compressible viscous flows, Journal of Computational Physics, Vol. 227, 2007, pp. 946-966.

[91] Kang, S., Iaccarino, G., Moin, P., Accurate Immersed-Boundary Recostructions for Viscous Flow Simulations, AIAA Journal, Vol. 47, No. 7, July 2009.

[92] Brehm, C., Hader, C., Fasel, H. F., Novel Immersed/Interface Method for the Compressible Navier-Stokes Equations, 50th AIAA Aerospace Sciences Meeting, AIAA 2012-1110, 09-12 January 2012, Nashville, Tennessee.

[93] Keistler, P. G., An Immersed Boundary Method for Supersonic Flow, 46th AIAA Aerospace Sciences Meeting and Exhibit, AIAA 2008-529, 7-10 January 2008, Reno, Nevada.

[94] Arina, R., Mohammadi, B., And Immersed Boundary Method for Aeroacoustics Problems, 14th AIAA/CEAS Aeroacoustics Conference, AIAA 2008-3003, 5-7 May 2-8, Vancouver, British Columbia, Canada.

[95] Seo, J. H., Mittal, R., A New Immersed Boundary Method for Aeroscoustics Prediction Around Complex Geometries, 40th Fluid Dynamics Conference and Exhibit, AIAA 2010-4434, 28 june - 1 july 2010, Chicago, Illinois.

[96] Nakahashi, K., Immersed Boundary Method for Compressible Euler Equations in the Building-Cube Method, 20th AIAA Computational Fluid Dynamics Conference, AIAA 2011-3386, 27-30 June 2011, Honolulu, Hawaii. 
[97] Balaras, E., Vanella, M., Adaptive Mesh Refinement Strategies for Immersed Boundary Methods, 47th AIAA Aerospace Sciences Meeting, AIAA 2009-162, 5-8 January 2009, Orlando, Florida.

[98] Wang, W., Pletcher, R., Large Eddy Simulation of Compressible Flows in a Rectangular Duct Containing Cylindrical Rods - an Immersed Boundary Treatment, 45th AIAA Aerospace Sciences Meeting and Exhibit, AIAA 2007-921, 8-11 January 2007, Reno, Nevada.

[99] Yang, X., He, G., Zhang, X., Towards Large-eddy Simulation of Turbulent Flows with Complex Geometric Boundaries Using Immersed Boundary Method, 48th AIAA Aerospace Sciences Meeting, AIAA 2010-708, 4-7 January 2010, Orlando, Florida.

[100] Takahashi, Y., Imamura, T., High Reynolds Number Steady State Flow Simulation using Immersed Boundary Method, 52nd Aerospace Sciences Meeting, DOI: 10.2514/6.2014-0228, 13.17 January, 2014.

[101] Capizzano, F., Turbulent Wall Model for Immersed Boundary Methods, AIAA Journal, DOI: 10.2514/1.J050466, Vol.49, No.11, November, 2011.

[102] Ishida, T., Nakahashi, K., Immersed Boundary Method for Compressible Turbulent Flow Computations in Building-Cube Method, 21st AIAA Computational Fluid Dynamics Conference, DOI: 10.2514/6.2013-2451, June 24-27, 2013.

[103] Zhong, G., Sun, X., New Simulation Strategy for an Oscillating Cascade in Turbomachinery Using Immersed-Boundary Method, Journal of Propulsion and Power, DOI: 10.2514/1.35347, Vol 25, No. 2, March-April 2009.

[104] Zheng, Z. C., Wei, Z., Zhang, N., Immersed-Boundary Simulation of Fast Pitching Airfoils, 40th Fluid Dynamics Conference and Exhibit, AIAA 2010-4280, 28 June - 1 july, Chicago, Illinous, 2010.

[105] Zhang, N., He, A., Ballard, B., Saxon, R., Schippers, W., Simulation of the Motion of a Hydro Turbine Using the Immersed-Boundary Method, 50th AIAA Aerospace Sciences Meeting, AIAA 2012-1234, 9-12 January, Nashville, Tennessee, 2012. 
[106] Onishi, K., Obayashi, S., Nakahashi, K., Tsubokura, M., Use of the Immersed Boundary Method within the Building Cube Method and its Application to Real Vehicle CAD Data, 21st AIAA Computational Fluid Dynamics Conference, DOI: 10.2514/6.20132713, 24-27 June, 2013.

[107] Tran, H. P., Plourde, F., Application of Immersed Boundary Technique in SRM Simulations: Toward new Insights, 21st AIAA Computational Fluid Dynamics Conference, DOI: 10.2514/6.2013-2714, 24-27 June, 2013.

[108] Bobenrieth Miserda, R. F., Lauterjung Q., R., Maldonado, A. L. P., Ribeiro, I.D., Godoy, K., Neto, O.G., Direct Computation of Noise Generated by Complex Geometries Using a High-Order Immersed Boundary Method, 15th AIAA/CEAS Aeroacoustics Conference, AIAA 2009-3181, 2009.

[109] Bobenrieth Miserda, R. F., Maldonado, A. L. P., Gutierrez, B., Simulation of the Cascade-Gust Interaction Problem Using a High-Order Immersed Boundary Method, 16th AIAA/CEAS Aeroacoustics Conference, AIAA 2010-3889, 2010.

[110] https://sr.grc.nasa.gov/public/project/48/

[111] Bobenrieth Miserda, R. F., Maldonado, A. L. P., Gutierrez, B., A Moving-Body High-Order Immersed Boundary Method for Computational Aeroacoustics, 17th AIAA/CEAS Aeroacoustics Conference, AIAA 2011-2753, 2011.

[112] Yee, H. C., Explicit and Implicit Multidimensional Compact High-Resolution ShockCapturing Methods: Formulation, Journal of Computational Physics, Vol. 131, 1997, pp. 216-232

[113] Tam, C., Webb, J., Dispersion-Relation-Preserving Finite Difference Schemes for Computational Acoustics, Journal of Computational Physics, 107, 262-281, 1993.

[114] Ducros, F., Laporte, F., Soulères, T., Guinot, V., Moinat, P., Caruelle, B., HighOrder Fluxes for Conservative Skew-Symmetric-like Schemes in Structured Meshes: Application to Compressible Flows, Journal of Computational Physics, Vol. 161, 2000, pp. $114-139$ 
[115] Giacché, D., Hynes, T, Baralon, S., Coupland J., Humphreys, N, Schwaller, P., Acoustic Optimization of Ultra-Low Count Bypass Outlet Guide Vanes, AIAA and CEAS 19th Aeroacosutics Conference, AIAA 2013-2295, Berlin, Germany, 2013.

[116] Jameson, A., Schmidt, W., Turkel, E., Numerical Solutions of the Euler Equations by Finite Volume Methods Using Runge-Kutta Time-Stepping Schemes, AIAA 14th Fluid and Plasma Dynamics Conference, AIAA-81-1259, 1981

[117] Mendonça, A. F. de, Simulação Numérica do Escoamento Transônico Laminar em Uma Base Bidimensional, Dissertação de Mestrado, Departamento de Engenharia Mecânica, Universidade de Brasília, 2004

[118] Tanehill, John C., Anderson, Dale A., Pletcher, Richard H., Computational Fluid Mechanics and Heat Transfer, Second Edition, Taylor and Francis, 1997

[119] Ruetsch, G., Fatica, M., CUDA Fortran for Scientists and Engineers: Best Practices for Efficient CUDA Fortran Programming, 1st edition, Elsevier, 2014

[120] Cheng, J., Grossman, M., McKercher, T., Professional CUDA C Programming, 1st edition, John Wiley \& Sons, 2014

[121] CUDA Fortran: Programming Guide and Reference Release 2015, The Portland Group, 2015

[122] Kester, J. D., Generation and Suppression of Combination Tone Noise From Turbofan Engines, Proceedings AGARD Fluid Dynamics Panel, Saint-Louis, France, 1969, Paper No. 19

[123] Sofrin, T. G., Pickett, G. F., Multiple Pure Tone Noise Generated by Fans at Supersonic Tip Speed, International Symposium on the Fluid Mechanics and Design of Turbomachinery, Pennsylvania State University, United States, 1970

[124] Hamming, R. W., Numerical Methods for Scientists and Engineers, Dover Publications, second edition, 1986

[125] Delfs, J., Basics of Aeroacoustics - Lecture Notes, Institut für Aerodynamik und Strömungstechnik, Technische Universität Braunschweig, Germany, October, 2012 
APPENDIX 


\section{BESSEL AND NEUMANN EIGENVALUES}




\begin{tabular}{|c|c|c|c|c|c|c|c|c|c|c|c|c|c|c|c|}
\hline \multirow{4}{*}{ 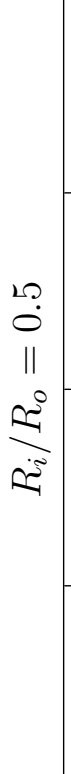 } & 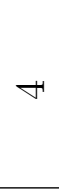 & 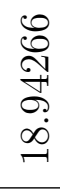 & 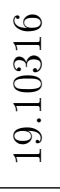 & 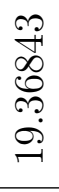 & 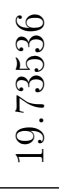 & 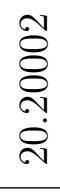 & 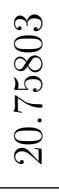 & 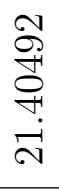 & 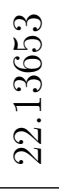 & 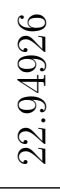 & 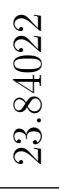 & 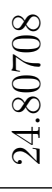 & 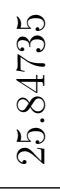 & 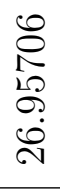 & $\begin{array}{l}\circ \\
\stackrel{0}{\Omega} \\
\stackrel{\sim}{N} \\
\stackrel{\infty}{\infty} \\
\stackrel{N}{0}\end{array}$ \\
\hline & $\infty$ & 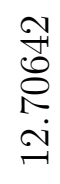 & 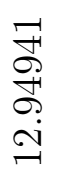 & 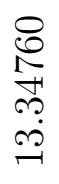 & 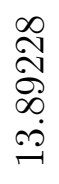 & 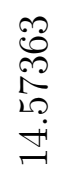 & 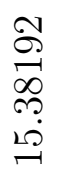 & $\begin{array}{l}\text { N } \\
\text { No } \\
\text { \% } \\
0 \\
0 \\
-1\end{array}$ & 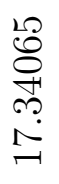 & 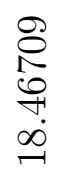 & $\begin{array}{l}10 \\
\stackrel{1}{0} \\
\infty \\
0 \\
0 \\
0 \\
0 \\
0\end{array}$ & 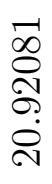 & 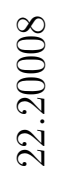 & 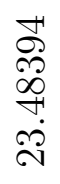 & 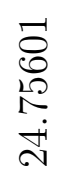 \\
\hline & $\sim$ & 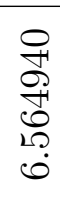 & 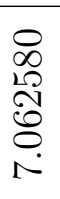 & $\begin{array}{l}\stackrel{0}{二} \\
\underset{\sigma}{\sigma} \\
\stackrel{\infty}{\infty} \\
\stackrel{N}{N}\end{array}$ & $\begin{array}{l}\stackrel{P}{+} \\
\underset{f}{+} \\
\infty \\
\infty \\
\infty \\
\infty\end{array}$ & $\begin{array}{l}0 \\
0 \\
\infty \\
10 \\
0 \\
0 \\
0\end{array}$ & 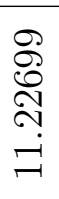 & 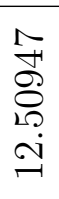 & 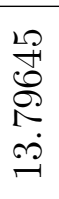 & 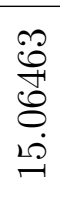 & 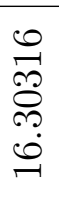 & 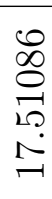 & $\begin{array}{l}0 \\
\stackrel{0}{\mathscr{N}} \\
\delta \\
0 \\
\infty \\
\infty\end{array}$ & 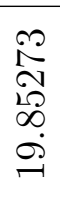 & $\begin{array}{l}\stackrel{2}{2} \\
\text { O } \\
\stackrel{2}{\rho} \\
\stackrel{\sim}{0}\end{array}$ \\
\hline & $\neg$ & 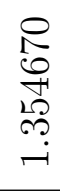 & 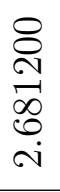 & $\begin{array}{l}0 \\
1 \\
1 \\
12 \\
2 \\
\infty \\
\infty\end{array}$ & 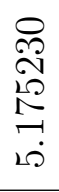 & $\begin{array}{l}8 \\
\infty \\
\infty \\
\infty \\
\infty \\
\infty \\
0\end{array}$ & 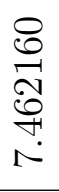 & 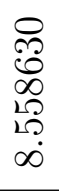 & 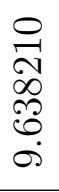 & $\begin{array}{l}0 \\
\stackrel{1}{N} \\
0 \\
0 \\
0 \\
0\end{array}$ & 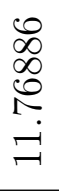 & 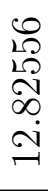 & 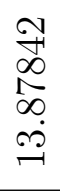 & 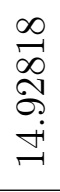 & $\begin{array}{l}10 \\
10 \\
10 \\
0 \\
0 \\
10\end{array}$ \\
\hline \multirow{4}{*}{$\begin{array}{l}0 \\
0 \\
11 \\
20 \\
\frac{2}{2}\end{array}$} & 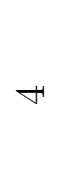 & 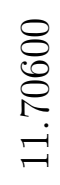 & 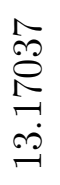 & $\begin{array}{l}10 \\
\infty \\
20 \\
\infty \\
20 \\
+ \\
+\end{array}$ & 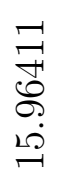 & 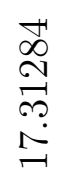 & 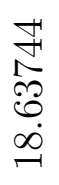 & 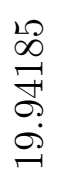 & 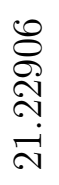 & $\begin{array}{l}\stackrel{ }{H} \\
\stackrel{1}{\circ} \\
\stackrel{0}{0} \\
\underset{N}{ }\end{array}$ & 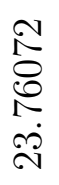 & 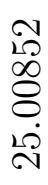 & $\begin{array}{l}\qquad 0 \\
\stackrel{0}{0} \\
\stackrel{0}{N} \\
\stackrel{0}{0} \\
\stackrel{1}{1}\end{array}$ & 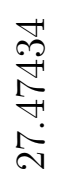 & 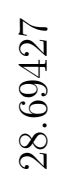 \\
\hline & $\infty$ & 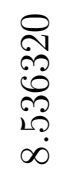 & 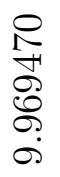 & 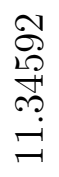 & 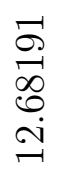 & 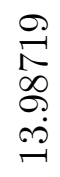 & $\begin{array}{l}\infty \\
\infty \\
\infty \\
\sim \\
\stackrel{0}{1} \\
\stackrel{1}{\sim}\end{array}$ & 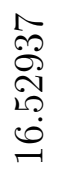 & $\begin{array}{l}\stackrel{j}{2} \\
\stackrel{N}{N} \\
\stackrel{N}{-1}\end{array}$ & 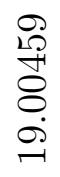 & 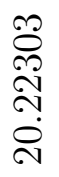 & 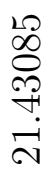 & 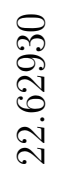 & 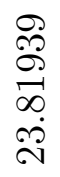 & 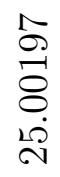 \\
\hline & $\sim$ & 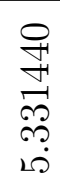 & 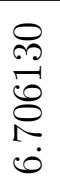 & 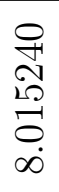 & 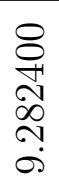 & $\begin{array}{l}0 \\
\infty \\
0 \\
0 \\
10 \\
0 \\
0\end{array}$ & 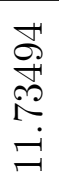 & 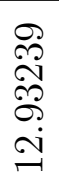 & 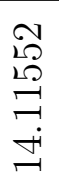 & 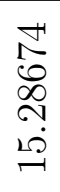 & 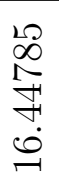 & 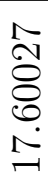 & $\begin{array}{l}\stackrel{8}{8} \\
\stackrel{10}{+1} \\
\stackrel{1}{\infty} \\
\infty\end{array}$ & $\begin{array}{l}\text { Nै } \\
\text { ஸे } \\
\infty \\
\infty \\
\infty \\
\infty \\
\infty\end{array}$ & 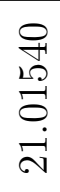 \\
\hline & $\neg$ & 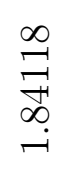 & 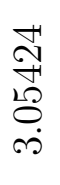 & $\begin{array}{l}\stackrel{\rho}{\sim} \\
\stackrel{\sim}{\sigma} \\
\underset{\sim}{\sim}\end{array}$ & 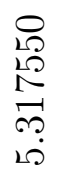 & 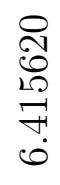 & 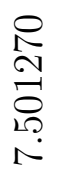 & $\begin{array}{l}\stackrel{P}{+} \\
\infty \\
1 \\
1 \\
1 \\
\infty\end{array}$ & 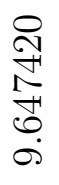 & 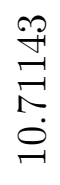 & 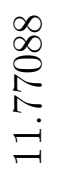 & 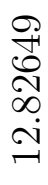 & 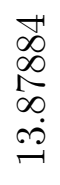 & 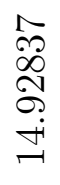 & $\begin{array}{l}\vec{H} \\
10 \\
1 \\
0 \\
10 \\
1\end{array}$ \\
\hline & $\frac{\tilde{\varepsilon}}{\tilde{\varepsilon}}$ & - & N & $\infty$ & 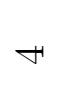 & 20 & 0 & $\Lambda$ & $\infty$ & $a$ & $\stackrel{ }{\rightarrow}$ & 二 & $\stackrel{\sim}{\sim}$ & $\stackrel{\oplus}{\sim}$ & $\underset{-}{\sqsupset}$ \\
\hline
\end{tabular}




\begin{tabular}{|c|c|c|c|c|c|c|c|c|c|c|c|c|c|c|c|}
\hline \multirow{2}{*}{ 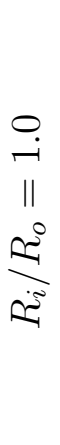 } & N & 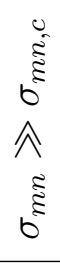 & $\begin{array}{l}0 \\
\tilde{E} \\
\text { है } \\
\hat{A} \\
\hat{E} \\
\text { है }\end{array}$ & $\begin{array}{l}0 \\
\tilde{E} \\
\text { है } \\
\hat{A} \\
\hat{E} \\
\text { है }\end{array}$ & 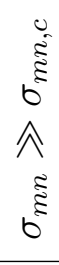 & 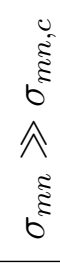 & 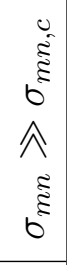 & 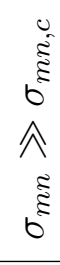 & $\begin{array}{l}0 \\
\hat{ह} \\
\text { हू } \\
\hat{A} \\
\hat{E} \\
0\end{array}$ & 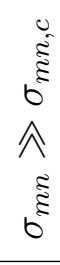 & 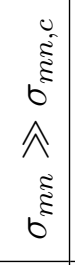 & 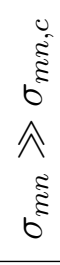 & 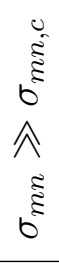 & $\begin{array}{l}0 \\
\text { हू है } \\
0 \\
\hat{\Lambda} \\
\text { हू } \\
0\end{array}$ & 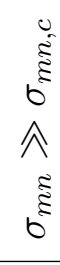 \\
\hline & 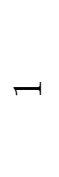 & $\begin{array}{l}8 \\
8 \\
8 \\
8 \\
\end{array}$ & $\begin{array}{c}\stackrel{8}{8} \\
\stackrel{8}{8} \\
\stackrel{\circ}{\text { ৩ }}\end{array}$ & $\begin{array}{l}\stackrel{8}{8} \\
8 \\
\stackrel{8}{8} \\
\text { r }\end{array}$ & $\begin{array}{l}\stackrel{8}{8} \\
\stackrel{8}{8} \\
\stackrel{+}{+}\end{array}$ & 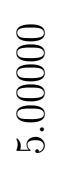 & $\begin{array}{l}8 \\
8 \\
8 \\
8 \\
0 \\
0\end{array}$ & $\begin{array}{l}\stackrel{8}{8} \\
\stackrel{8}{8} \\
\stackrel{\circ}{\circ}\end{array}$ & 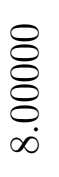 & $\begin{array}{l}\stackrel{8}{8} \\
\stackrel{8}{8} \\
\stackrel{8}{\circ} \\
\text {. }\end{array}$ & 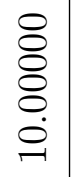 & 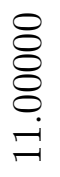 & $\begin{array}{l}\stackrel{8}{8} \\
\stackrel{8}{8} \\
\varrho \\
\text { ำ } \\
\end{array}$ & 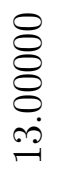 & 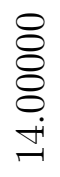 \\
\hline \multirow{2}{*}{ 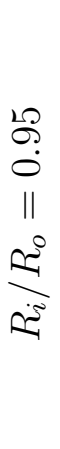 } & N & 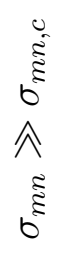 & 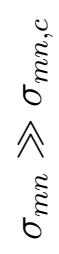 & 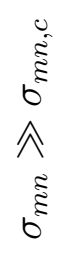 & $\begin{array}{l}0 \\
\text { हू है } \\
0 \\
\hat{\wedge} \\
\text { हू } \\
0\end{array}$ & 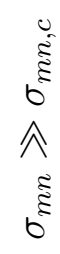 & 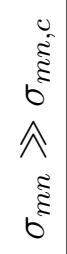 & 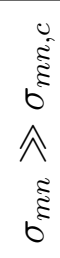 & $\begin{array}{l}0 \\
\text { हू ह } \\
0 \\
\hat{\wedge} \\
\text { हू } \\
0\end{array}$ & $\begin{array}{l}0 \\
\text { है है } \\
0 \\
\hat{\wedge} \\
\text { है } \\
0\end{array}$ & 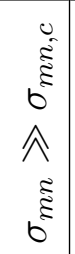 & $\begin{array}{l}0 \\
\text { हू है } \\
0 \\
\hat{\wedge} \\
\text { हू } \\
0\end{array}$ & 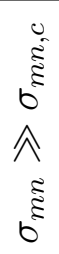 & $\begin{array}{l}0 \\
\text { हू है } \\
0 \\
\hat{\wedge} \\
\text { हू } \\
0\end{array}$ & 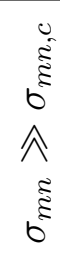 \\
\hline & - & 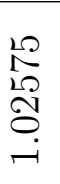 & 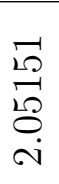 & $\begin{array}{l}\stackrel{0}{N} \\
\text { N } \\
\text { ○ } \\
\text { i }\end{array}$ & 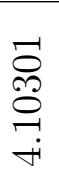 & 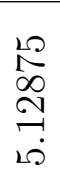 & 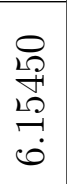 & $\begin{array}{l}\stackrel{\Re}{\stackrel{2}{O}} \\
\stackrel{\infty}{\infty} \\
\stackrel{\sim}{\sim}\end{array}$ & 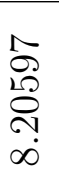 & 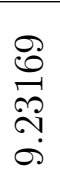 & 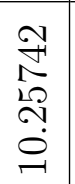 & 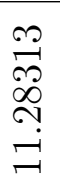 & 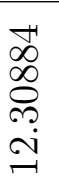 & 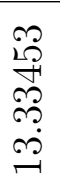 & 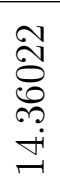 \\
\hline \multirow{4}{*}{$\begin{array}{c}0 \\
0 \\
\| \\
11 \\
\frac{0}{20} \\
20\end{array}$} & $\infty$ & 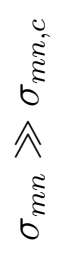 & 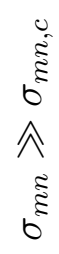 & 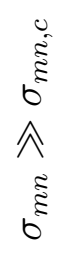 & $\begin{array}{l}0 \\
\tilde{E} \\
\text { हू } \\
\hat{\wedge} \\
\hat{E} \\
0\end{array}$ & 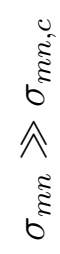 & 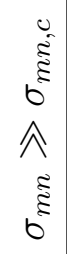 & $\begin{array}{l}0 \\
\tilde{E} \\
\text { है } \\
\hat{\Lambda} \\
\text { हू } \\
0\end{array}$ & $\begin{array}{l}0 \\
\text { हू ह } \\
0 \\
\hat{\wedge} \\
\text { हू } \\
0\end{array}$ & $\begin{array}{l}0 \\
\text { है है } \\
0 \\
\hat{\Lambda} \\
\text { है } \\
0\end{array}$ & 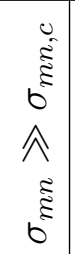 & $\begin{array}{l}0 \\
\text { हू हू } \\
0 \\
\hat{\wedge} \\
\text { हू } \\
0\end{array}$ & 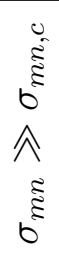 & 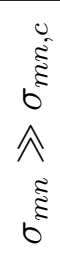 & 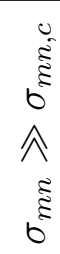 \\
\hline & $N$ & $\begin{array}{l}\infty \\
\infty \\
0 \\
\stackrel{7}{7} \\
\stackrel{\infty}{+}\end{array}$ & 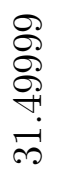 & 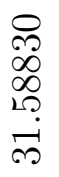 & 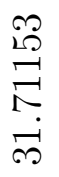 & 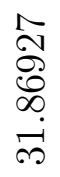 & 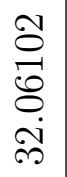 & $\begin{array}{l}\Omega \\
\vec{D} \\
\infty \\
\sim \\
\text { N. } \\
\text { ஸे }\end{array}$ & 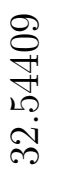 & $\begin{array}{l}20 \\
\infty \\
\text { ஸे } \\
\infty \\
\infty \\
\text { ஸे }\end{array}$ & & 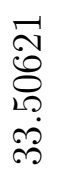 & $\begin{array}{l}\infty \\
0 \\
\infty \\
\infty \\
\dot{\infty} \\
\dot{\infty}\end{array}$ & 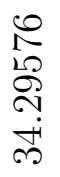 & 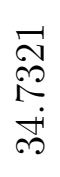 \\
\hline & $\neg$ & 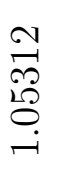 & 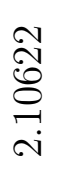 & 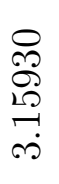 & $\begin{array}{l}\stackrel{\Re}{2} \\
\stackrel{2}{\sim} \\
\stackrel{\sim}{\sim}\end{array}$ & 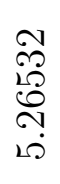 & 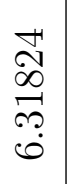 & $\begin{array}{l}\stackrel{2}{0} \\
\stackrel{\Gamma}{N} \\
\stackrel{\infty}{N}\end{array}$ & 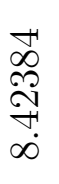 & 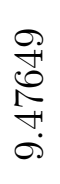 & 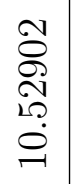 & 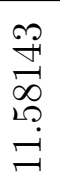 & 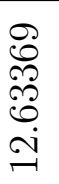 & 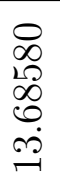 & $\begin{array}{l}\stackrel{+}{N} \\
\text { 足 } \\
\stackrel{+}{+}\end{array}$ \\
\hline & $\frac{\xi}{ह}$ & $-r$ & N & $\infty$ & $\forall$ & 10 & 0 & $N$ & $\infty$ & $a$ & $\stackrel{ }{\ominus}$ & 그 & $\stackrel{\sim}{\sim}$ & $\stackrel{m}{=}$ & $\rightleftarrows$ \\
\hline
\end{tabular}




\section{NOISE GENERATED \\ AERODYNAMICALLY}

Consider the surface $S(t)$ and the volume $V(t)$ of a given arbitrary body. This body is immersed in a sufficiently smooth fluid domain $V_{f}$ that moves continuously in space. An arbitrary function $f(\mathbf{x}, t)$ is then introduced such that:

$$
f(\mathbf{x}, t)=\left\{\begin{array}{l}
<0 \text { if } \mathbf{x} \in V(t), \\
=0 \text { if } \mathbf{x} \in S(t), \\
>0 \text { if } \mathbf{x} \notin V(t),
\end{array}\right.
$$

where the given condition refers to continuity, and the existence of the field where the surface is denoted by the isovalue for $f(\mathbf{x}, t)$. Now multiplying the function that defines the given surface by the Heaviside step function $H(f)$, a value of $H(f)=0$ is given by the subdomain where there is not fluid. At the fluid region $H(f)=1$. By doing this, only regions where there is fluid are considered in the following calculations.

Defining the body surface normal vector as [63]:

$$
\mathbf{n}(\mathbf{x}, t)=\left.\frac{\nabla f}{|\nabla f|}\right|_{f=0},
$$

where the body may be described with parametric coordinates fixed to its surface; $(t, \mu, \lambda)$. Taking a given point $\mathbf{x}_{s}(t)$ of the surface as $\mu$ and $\lambda$ of constant values, that moves at a given speed $\mathbf{U}=\dot{\mathbf{x}}_{s}$, we then have $f\left(\mathbf{x}_{s}(t), t\right)=0$. As a consequence of this mathematical relation, the material derivative of the function $f(t)$ at the surface is given by:

$$
\frac{D f}{D t}=\frac{\partial f}{\partial t}+\dot{\mathbf{x}}_{s} \cdot \nabla f=\frac{\partial f}{\partial t}+(\mathbf{U} \cdot \mathbf{n})|\nabla f|=0,
$$

as a consequence for the Heaviside step function:

$$
\frac{\partial}{\partial t}(H(f))=-\mathbf{U} \cdot \nabla H(f) .
$$

With all the mathematical relations already defined at the surface of the body immersed in fluid, we define the governing equations 2.8 and 2.9 in the main body of this thesis, at 
the fluid where the body is immersed at and without body forces:

$$
\begin{array}{r}
H(f)\left[\frac{\partial \rho}{\partial t}+\nabla \cdot(\rho \mathbf{u})\right]=0 \\
H(f)\left[\frac{\partial \rho \mathbf{u}}{\partial t}+\nabla \cdot(\rho \mathbf{u} \otimes \mathbf{u}-\tau+p \mathbf{I}) \cdot\right]=0
\end{array}
$$

By doing this the governing equations are already satisfied in all of the domain, including inside the body that is immersed in the fluid. Rearranging the terms to include $H(f)$ inside the derivatives (chain rule) and by applying the linearization decomposition:

$$
\begin{array}{r}
\frac{\partial}{\partial t}\left[\rho^{\prime} H(f)\right]+\nabla \cdot[\rho \mathbf{u} H(f)]=\left[\rho_{0} \mathbf{U}+\rho(\mathbf{u}-\mathbf{U})\right] \cdot \nabla H(f), \\
\frac{\partial}{\partial t}[\rho \mathbf{u} H(f)]+\nabla \cdot[(\rho \mathbf{u u}+p \mathbf{I}-\tau) H(f)]=[\rho \mathbf{u}(\mathbf{u}-\mathbf{U})+p \mathbf{I}-\tau] \cdot \nabla H(f) .
\end{array}
$$

Equations II.6 are nothing more than the the Navier-Stokes equation under the generalized equations form. Now taking the temporal derivative of the first equation and the divergent of the second one, then subtracting one from the other and adding the term $-c_{0}{ }^{2} \nabla^{2}\left(\rho^{\prime} H(f)\right)$ to both sides of the resulting equation. Finally applying the given identity for the Heaviside function $\nabla H(f)=\mathbf{n}|\nabla f| \delta(f)$ :

$$
\begin{aligned}
& \frac{\partial^{2}}{\partial t^{2}}\left[\rho^{\prime} H(f)\right]-c_{0}^{2} \nabla^{2}\left(\rho^{\prime} H(f)\right)=\quad \nabla \cdot\left[\nabla \cdot\left[\rho \mathbf{u u}-\tau+\left(p^{\prime}-c_{0}^{2} \rho^{\prime}\right) \mathbf{I}\right] H(f)\right] \\
& +\frac{\partial}{\partial t}\left[\left(\rho(\mathbf{u}-\mathbf{U})+\rho_{0} \mathbf{U}\right) \cdot \mathbf{n}|\nabla f| \delta(f)\right] \\
& -\nabla \cdot\left[\left(\rho \mathbf{u}(\mathbf{u}-\mathbf{U})+p^{\prime} \mathbf{I}-\tau\right) \cdot \mathbf{n}|\nabla f| \delta(f)\right] .
\end{aligned}
$$

The equation above is the Ffowcs Williams-Hawkings equation [52]. It is the Navier-Stokes equations in the form of the wave equation. The Ffowcs Williams-Hawkings equation may be use as an acoustic analogy. By comparing it with the wave equation, we may characterize the aeroacoustic source. The Ffowcs Williams-Hawkings equation is very similar to the wave equation obtained previously but without the advective term and with the right hand side sources that makes it a non-homogeneous equation. This is known as the wave equation applied to the Green's function:

$$
\left(\frac{1}{c_{0}^{2}} \frac{\partial^{2}}{\partial t^{2}}-\nabla^{2}\right) G=\delta(\mathbf{x}-\mathbf{y}) \delta(t-\tau) .
$$

Several Green's functions exist depending on the governing equation to which they are applied to. When applied to the non-homogeneous wave equation, the solution of the threedimensional free propagation in a quiescent fluid is the most used. The Green's function 
for this equation is given by:

$$
\left\{\begin{array}{l}
G=0 \text { for } t<\tau \\
G(\mathbf{x}, \mathbf{y}, t-\tau)=\frac{1}{4 \pi|\mathbf{x}-\mathbf{y}|} \delta\left(t-\tau-\frac{|\mathbf{x}-\mathbf{y}|}{c_{0}}\right) \text { para } t \geq \tau .
\end{array}\right.
$$

Consider equation II.9 for a generalized source distributed along the time and space coordinates:

$$
\left(\frac{1}{c_{0}^{2}} \frac{\partial^{2}}{\partial t^{2}}-\nabla^{2}\right) G=F(\mathbf{x}, t)=\int_{0}^{t} \int_{\infty} F(\mathbf{y}, \tau) \delta(\mathbf{x}-\mathbf{y}) \delta(\tau-t) d V d \tau
$$

for the wave equation for the pressure fluctuations, the free field propagation solution is [64]:

$$
p^{\prime}(\mathbf{x}, t)=\frac{1}{4 \pi} \int_{0}^{t} \int_{\infty} \frac{F(\mathbf{y}, \tau)}{|\mathbf{x}-\mathbf{y}|} \delta\left(t-\tau-\frac{|\mathbf{x}-\mathbf{y}|}{c_{0}}\right) d V d \tau
$$

or in the retarded potential, where the Dirac delta is evaluated when different from zero and applied in $F(\mathbf{y}, \tau)$ when $\tau=t-\frac{|\mathbf{x}-\mathbf{y}|}{c_{0}}$ :

$$
p^{\prime}(\mathbf{x}, t)=\frac{1}{4 \pi} \int_{\infty} \frac{F\left(\mathbf{y}, t-\frac{|\mathbf{x}-\mathbf{y}|}{c_{0}}\right)}{|\mathbf{x}-\mathbf{y}|} d V .
$$

The retarded potential is the pressure signal on the position $\mathbf{x}$ at a given instant $t$ as a linear superposition of the source terms contribution through space at $\mathbf{y}$ that radiated at a previous time $t-\frac{|\mathbf{x}-\mathbf{y}|}{c_{0}}$, where $\frac{|\mathbf{x}-\mathbf{y}|}{c_{0}}$ is the time needed for the wave to travel from the source position $\mathbf{y}$ to the observer position $\mathbf{x}$.

Applying the Green's function of generalized sources into the Ffowcs Williams-Hawkings equation and noting that the source terms are evaluated at the retarded potential $t-\frac{|\mathbf{x}-\mathbf{y}|}{c_{0}}$ :

$$
\begin{aligned}
p^{\prime}(\mathbf{x}, t)=\frac{1}{4 \pi} \int_{\infty} \frac{\nabla \cdot\left[\nabla \cdot\left[\left(\rho \mathbf{u u}-\tau+\left(p^{\prime}-c_{0}^{2} \rho^{\prime}\right) \mathbf{I}\right) H(f)\right]\right]}{|\mathbf{x}-\mathbf{y}|} d V \\
+\frac{1}{4 \pi} \int_{\infty} \frac{\frac{\partial}{\partial t}\left[\left(\rho(\mathbf{u}-\mathbf{U})+\rho_{0} \mathbf{U}\right) \cdot \mathbf{n}|\nabla f| \delta(f)\right]}{|\mathbf{x}-\mathbf{y}|} d V \\
\quad-\frac{1}{4 \pi} \int_{\infty} \frac{\nabla \cdot\left[\left(\rho \mathbf{u}(\mathbf{u}-\mathbf{U})+p^{\prime} \mathbf{I}-\tau\right) \cdot \mathbf{n}|\nabla f| \delta(f)\right]}{|\mathbf{x}-\mathbf{y}|} d V .
\end{aligned}
$$

By evaluating the term $\delta(f)$, where it is not zero only at the surface and the term $|\nabla f| \delta(f)$ 
is directly related to the integration at the body surface $S(t)$ :

$$
\begin{aligned}
p^{\prime}(\mathbf{x}, t)=\frac{1}{4 \pi} \int_{\infty} \frac{\nabla \cdot\left[\nabla \cdot\left[\left(\rho \mathbf{u u}-\tau+\left(p^{\prime}-c_{0}^{2} \rho^{\prime}\right) \mathbf{I}\right) H(f)\right]\right]}{|\mathbf{x}-\mathbf{y}|} d V \\
+\frac{1}{4 \pi} \oint_{S(t)} \frac{\frac{\partial}{\partial t}\left[\left(\rho(\mathbf{u}-\mathbf{U})+\rho_{0} \mathbf{U}\right) \cdot \mathbf{n}\right]}{|\mathbf{x}-\mathbf{y}|} d S \\
\quad-\frac{1}{4 \pi} \oint_{S(t)} \frac{\nabla \cdot\left[\left(\rho \mathbf{u}(\mathbf{u}-\mathbf{U})+p^{\prime} \mathbf{I}-\tau\right) \cdot \mathbf{n}\right]}{|\mathbf{x}-\mathbf{y}|} d S .
\end{aligned}
$$

Taking into account that the body is rigid and that there is no fluid passing through its surface, i.e., $\mathbf{U}=\mathbf{u}$, the Ffowcs Williams-Hawkings equation applied into a threedimensional free field propagation is then obtained [125]:

$$
\begin{aligned}
p^{\prime}(\mathbf{x}, t)=\frac{1}{4 \pi} \int_{\infty} \frac{\nabla \cdot\left[\nabla \cdot\left[\left(\rho \mathbf{u u}-\tau+\left(p^{\prime}-c_{0}^{2} \rho^{\prime}\right) \mathbf{I}\right) H(f)\right]\right]}{|\mathbf{x}-\mathbf{y}|} d V \\
\quad+\frac{1}{4 \pi} \oint_{S(t)} \frac{\frac{\partial}{\partial t}\left[\left(\rho_{0} \mathbf{U}\right) \cdot \mathbf{n}\right]}{|\mathbf{x}-\mathbf{y}|} d S-\frac{1}{4 \pi} \oint_{S(t)} \frac{\nabla \cdot\left[\left(p^{\prime} \mathbf{I}-\tau\right) \cdot \mathbf{n}\right]}{|\mathbf{x}-\mathbf{y}|} d S .
\end{aligned}
$$

Equation II.15 describes the propagated acoustic signal $p^{\prime}$ at a given point $\mathbf{x}$ along a physical time $t$ when aeroacoustic sources are concerned. The right hand side integrals describes the noise sources acoording with the flow interactions.

Mathematically the source terms may be characterized as polar sources according to the mathematical similarity between the source term and the kind of pole. On the right hand side of equation II.15 the first term is a quadrupole noise source, due to the second order space derivative. The second term is a monopole kind of noise source, because there is not a space derivative. The third term represents the dipole noise source, due to its first order space derivative. The noise origin of each source term is also distinct. The first term is due to the fluid flow only with its three components:

- $\rho \mathbf{u u}$ : Reynolds stress tensor; related to the velocity fluctuations in the fluid. Commonly associated with turbulence. Also occurs in highly nonlinear flows that are not necessarily turbulent.

- $-\tau$ : noise viscous dissipation source, usually neglected due to its low influence in dissipation of acoustic fluctuations.

- $p^{\prime}-c_{0}^{2} \rho^{\prime}$ : non-isentropic compressibility term. Related to deviation of reversible processes to irreversibility due to high frequency or high amplitude fluctuations $\left(p^{\prime} \sim\right.$ $\left.p_{0}\right)$. 
The Ffowcs Williams-Hawkings equation with only the quadrupole noise sources is commonly known as the Lighthill analogy. The noise source terms with surface intgrals in equation II.15 are due to the immersed bodies in the flow that has an aerodynamic field along its body generating noise. The monopole source is due to the noise related to the body thickness or the fact that the body is dislocating fluid along the flow. The dipole noise source is related to the aerodynamic loading along the body. This noise source depends on the stress distribution along the body. It is commonly known as loading noise source [125]. 


\section{MECHANICAL ENERGY EQUATION}

By taking the scalar product between $\mathbf{u}$ and the Cauchy's equation with body forces:

$$
\rho \mathbf{u} \cdot \frac{D \mathbf{u}}{D t}=\mathbf{u} \cdot \nabla \cdot \sigma+\mathbf{u} \cdot \rho \mathbf{f}_{e}
$$

The body force can be represented as a potential field:

$$
\mathbf{f}_{e}=-\nabla \phi
$$

Substituting equation III.1 into equation III.2:

$$
\rho \mathbf{u} \cdot \frac{D \mathbf{u}}{D t}=\mathbf{u} \cdot \nabla \cdot \sigma-\rho(\mathbf{u} \cdot \nabla \phi)
$$

The material derivative of the body force term is given by:

$$
\frac{D \phi}{D t}=\frac{\partial \phi}{\partial t}+\mathbf{u} \cdot \nabla \phi
$$

where the potential term of a constant body force is:

$$
\frac{D \phi}{D t}=\mathbf{u} \cdot \nabla \phi
$$

Substituting equation III.5 into equation III.3:

$$
\rho \mathbf{u} \cdot \frac{D \mathbf{u}}{D t}=\mathbf{u} \cdot \nabla \cdot \sigma-\rho \frac{D \phi}{D t}
$$

Then, rearranging the right hand side of equation III.6 in the kinetic energy form:

$$
\rho \frac{D}{D t}\left(\frac{1}{2} \mathbf{u}^{2}\right)=-\rho \frac{D \phi}{D t}+\mathbf{u} \cdot \nabla \cdot \sigma
$$

rearranging the material derivative term of the body force potential on the left hand side:

$$
\rho \frac{D}{D t}\left(\frac{1}{2} \mathbf{u}^{2}+\phi\right)=\mathbf{u} \cdot \nabla \cdot \sigma .
$$

Equation III.8 is the non-conservative differential form of the specific mechanical energy. The left hand side represents the kinetic and potential energies variation of a particle in the flow counter balanced by the work done by surface forces on the right hand side. 


\section{VORTICITY GENERATION}

In the rotor and stator interaction case, some of the governing equations are the Euler equations for the linear momentum and sometimes the viscosity is characterized as the only vorticity generation mechanism. The following arguments deny this assertion and deduce other vorticity mechanisms according to the numerical results presented in chapter 5 .

The liner momentum equation for an inviscid fluid under the non-conservative form may be rewritten as follows:

$$
\frac{\partial \mathbf{u}}{\partial t}+(\mathbf{u} \cdot \nabla) \mathbf{u}=-\frac{1}{\rho} \nabla p
$$

From the following vector identity:

$$
\mathbf{u} \cdot \nabla \mathbf{u}=\frac{1}{2} \nabla \mathbf{u}^{2}-\mathbf{u} \times \nabla \times \mathbf{u}
$$

substituting equation IV.2 into equation IV.1:

$$
\frac{\partial \mathbf{u}}{\partial t}+\frac{1}{2} \nabla \mathbf{u}^{2}-\mathbf{u} \times \nabla \times \mathbf{u}=-\frac{1}{\rho} \nabla p .
$$

By applying the curl operator into equation IV.3:

$$
\nabla \times \frac{\partial \mathbf{u}}{\partial t}+\nabla \times \frac{1}{2} \nabla \mathbf{u}^{2}-\nabla \times(\mathbf{u} \times \nabla \times \mathbf{u})=-\frac{1}{\rho}(\nabla \times \nabla p)-\frac{1}{\rho^{2}} \nabla p \times \nabla \rho .
$$

Also by vector identities, the second and the fourth terms from equation IV.3 are equal to zero:

$$
\nabla \times \frac{\partial \mathbf{u}}{\partial t}-\nabla \times(\mathbf{u} \times \nabla \times \mathbf{u})=-\frac{1}{\rho^{2}} \nabla p \times \nabla \rho
$$

Consider the following vector identity:

$$
\nabla \times(\mathbf{u} \times \nabla \mathbf{u})=-(\nabla \times \mathbf{u})(\nabla \cdot \mathbf{u})+(\nabla \times \mathbf{u}) \cdot \nabla \mathbf{u}-\mathbf{u} \cdot \nabla(\nabla \times \mathbf{u}) .
$$

Substituting equation IV.6 into equation IV.5:

$$
\nabla \times \frac{\partial \mathbf{u}}{\partial t}+(\nabla \times \mathbf{u})(\nabla \cdot \mathbf{u})-(\nabla \times \mathbf{u}) \cdot \nabla \mathbf{u}+\mathbf{u} \cdot \nabla(\nabla \times \mathbf{u})=-\frac{1}{\rho^{2}} \nabla p \times \nabla \rho .
$$

Rearranging the terms:

$$
\frac{\partial}{\partial t}(\nabla \times \mathbf{u})+\mathbf{u} \cdot \nabla(\nabla \times \mathbf{u})=(\nabla \times \mathbf{u}) \cdot \nabla \mathbf{u}-(\nabla \times \mathbf{u})(\nabla \cdot \mathbf{u})-\frac{1}{\rho^{2}} \nabla p \times \nabla \rho .
$$


In an alternative way, using a more compact notation the left hand side of equation IV.8 can be written as:

$$
\frac{\mathrm{D}}{\mathrm{D} t}(\nabla \times \mathbf{u})=(\nabla \times \mathbf{u}) \cdot \nabla \mathbf{u}-(\nabla \times \mathbf{u})(\nabla \cdot \mathbf{u})-\left(\frac{1}{\rho^{2}}\right) \nabla p \times \nabla \rho
$$

For two-dimensional flows, the vorticity equation can be simplified:

$$
\frac{\mathrm{D}}{\mathrm{D} t}(\nabla \times \mathbf{u})=-(\nabla \times \mathbf{u})(\nabla \cdot \mathbf{u})-\left(\frac{1}{\rho^{2}}\right) \nabla p \times \nabla \rho
$$

due to the orthogonality between $\nabla \times \mathbf{u}$, which is normal to the plane of the two-dimensional flow and $\nabla \mathbf{u}$, that is in the flow field plane. In equation IV.10 the first term of the right hand side is the contribution to the vorticity variation in a fluid element that moves along the flow, where $\nabla \cdot \mathbf{u}$ is the rate of change of volume of that fluid element.

The last term of the right hand side of equation IV.10 is the contributing term of the vorticity generation on a fluid element on the flow due to the local pressure gradient $\nabla p$. By using the perfect gas equation 2.13, this term can be rewritten as:

$$
\nabla p=\nabla(\rho \mathcal{R} T)=\mathcal{R} T \nabla \rho+\mathcal{R} \rho \nabla T
$$

showing that if the pressure gradient of a given point is non zero, the second source term from equation IV.10 will be usually non-zero unless the temperature gradient is also equal to zero. The two-dimensional cases of rotor and stator interaction show vorticity generation due to the pressure gradient term resulting in vorticity wakes of varied intensity depending on the flow case. It is also important to note that the pressure gradient source term may be equal to zero, but with non zero values for $\nabla T$, if it has the same direction as $\nabla \rho$, as usually happens in acoustic wave propagation phenomena. 